\title{
Anti-inflammatory and pro-resolving role of mast cells during resolution of local inflammation.
}

\author{
Dissertation \\ zur Erlangung des Doktorgrades \\ der Naturwissenschaften \\ Vorgelegt beim Fachbereich 14 \\ der Johann Wolfgang Goethe - Universität \\ in Frankfurt am Main \\ von \\ Lisa Kornstädt \\ aus Sinsheim
}

Frankfurt (2021)

(D30) 
Vom Fachbereich 14 der

Johann Wolfgang Goethe - Universität als Dissertation angenommen.

Dekan: $\quad$ Prof. Dr. Clemens Glaubitz

Gutachter: Prof. Dr. Dr. Achim Schmidtko

Prof. Dr. Dr. Klaus Scholich

Datum der Disputation: 14.06.2021 


\section{Erklärung}

Ich erkläre hiermit, dass ich mich bisher keiner Doktorprüfung im MathematischNaturwissenschaftlichen Bereich unterzogen habe.

Ich erkläre hiermit, dass ich die vorgelegte Dissertation über

"Anti-inflammatory and pro-resolving role of mast cells during resolution of local inflammation"

selbständig angefertigt und mich anderer Hilfsmittel als der in ihr angegebenen nicht bedient habe, insbesondere, dass alle Entlehnungen aus anderen Schriften mit Angabe der betreffenden Schrift gekennzeichnet sind. Ich versichere, die Grundsätze der guten wissenschaftlichen Praxis beachtet, und nicht die Hilfe einer kommerziellen Promotionsvermittlung in Anspruch genommen zu haben. 


\section{Declaration}

Except where stated otherwise by reference or acknowledgment, the work presented was generated by myself under the supervision of my advisors during my doctoral studies. All contributions from colleagues are explicitly referenced in the thesis. The material listed below was obtained in the context of collaborative research:

Fig. 8: Gating strategy for FACS sorting of mature BMMCs for RNA sequencing. Fluorescence-activated cell sorting (FACS) was performed by Andreas Weigert and Praveen Mathor (Department of Patho Biochemisty, University Hospital Goethe University Frankfurt). Samples were prepared and further processed by me.

Fig. 11: Distinct lipid profiles and chemokine levels after injection of zymosan or LPS. LC-MS experiments including sample preparation and analysis were performed by Nerea Fereirós, Lisa Hahnefeld, and Daniel Kratz (Institute of Clinical Pharmacology, University Hospital Goethe University Frankfurt). Multiplex Immunoassay of plasma samples including sample preparation and analysis of the data was performed by Sandra Pierre (Institute of Clinical Pharmacology, University Hospital Goethe University Frankfurt).

Fig. 12: Mast cells are located in the anti-inflammatory region during the resolution phase, surrounding the zymosan core of the inflammation. Fig. 12A: Behavioural experiment was performed by me. Fig. 12B: MELC was performed by Tim Schäufele and Anja Kolbinger (Institute of Clinical Pharmacology, University Hospital Goethe University Frankfurt). Tissue samples were prepared by me.

Fig. 13: Identification and characterization of immune cell phenotypes in MELC images of inflamed paws. MELC and MELC analysis was performed by Tim Schäufele and Anja Kolbinger (Institute of Clinical Pharmacology, University Hospital Goethe University Frankfurt). Tissue samples were prepared by me.

Fig. 14: Efferocytosis increases during resolution of inflammation. MELC and MELC analysis was performed by Tim Schäufele and Anja Kolbinger (Institute of Clinical Pharmacology, University Hospital Goethe University Frankfurt). Tissue samples were prepared by me.

Fig. 15: Mast cells are located in anti-inflammatory neighbourhood during resolution of zymosan-induced inflammation. MELC and MELC analysis was performed by Tim Schäufele and Anja Kolbinger (Institute of Clinical Pharmacology, University Hospital Goethe University Frankfurt). Tissue samples were prepared by me. 
Fig. 17: M1 macrophage phagocytosis of neutrophils is reduced in mast cell-deficient Mcpt5DTA Cre ${ }^{+}$mice compared to Cre- control mice. MELC and MELC analysis was performed by Tim Schäufele and Anja Kolbinger (Institute of Clinical Pharmacology, University Hospital Goethe University Frankfurt). Tissue samples were prepared by me.

Fig. 18: Decreased Ly6G signal in M1 macrophages in paws of mast cell-deficient Mcpt5DTA Cre ${ }^{+}$mice. MELC and MELC analysis was performed by Tim Schäufele and Anja Kolbinger (Institute of Clinical Pharmacology, University Hospital Goethe University Frankfurt). Tissue samples were prepared by me.

Fig. 21: Levels of prostanoids during local inflammation in Mcpt5-DTA Cre mice. Prostanoids were analysed by Yannick Schreiber and Dominique Thomas. (Institute of Clinical Pharmacology, University Hospital Goethe University Frankfurt). Sample preparation was performed by me, as well as the statistical analysis and further evaluation.

Fig. 24: Chemokines and prostanoids released by mast cells after induction with zymosan or LPS. Fig. 24A: Chemokines were analysed by me. Fig. 24B: Prostanoids were analysed by Yannick Schreiber and Dominique Thomas. (Institute of Clinical Pharmacology, University Hospital Goethe University Frankfurt). Sample preparation was performed by me, as well as the statistical analysis and further evaluation.

Fig. 26: Release of IL-10 does not depend on IgE-mediated degranulation but on COX signaling. $\mathrm{PGE}_{2}$ and histamine were analysed by Yannick Schreiber and Dominique Thomas. (Institute of Clinical Pharmacology, University Hospital Goethe University Frankfurt). IL-10 was analysed by me. Sample preparation was performed by me, as well as the statistical analysis and further evaluation.

Fig. 28: Differential expression analysis shows that most genes are commonly regulated after treatment for $24 \mathrm{~h}$ or $48 \mathrm{~h}$ compared to untreated samples. Differential expression analysis of RNA sequencing data was performed by Stefanie Ebersberger (Institut für Molekulare Biologie, Johannes Gutenberg-Universität Mainz) and Ingo Ebersberger (Institute of Cell Biology and Neuroscience, Goethe University Frankfurt, Germany). Sample preparation and sequencing was performed by me.

Fig. 31: IFN- $\beta$ is produced by mast cells in vitro and in vivo. Fig 31A-C: Experiments and analysis were performed by me. Fig 31D: MELC was performed by Tim Schäufele and Anja Kolbinger (Institute of Clinical Pharmacology, University Hospital Goethe University Frankfurt). Tissue samples were prepared by me. 
Whenever a figure, table or text is identical to a previous publication, it is stated explicitly in the thesis that copyright permission and/or co-author agreement has been obtained. The following parts of the thesis have been previously published:

- Chapter 2.2 "Methods" - Figure 6 (Hahnefeld \& Kornstädt et al., 2021)

- Chapter 2.2 "Methods" - Figure 7-8 (Kornstädt et al., 2021)

- Chapter 3.1 "Comparison of two locally induced inflammation models" - Figure 9-11 (Hahnefeld \& Kornstädt et al., 2021)

- Chapter 3.2 "Role of mast cells during resolution of local inflammation" - Figure 12-21 (Kornstädt et al., 2021)

- Chapter 3.3 "Identification of mediators produced by mast cells" - Figure 22-24, Figure 28-31, Figure 33-34 (Kornstädt et al., 2021) 


\title{
Table of contents
}

\author{
Erklärung III \\ Declaration $\mathrm{V}$
}

Abstract XIII

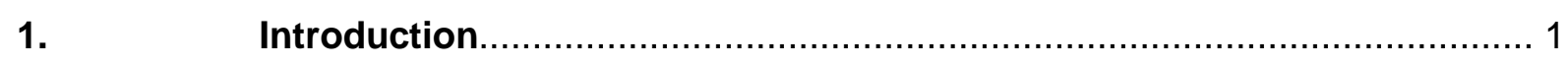

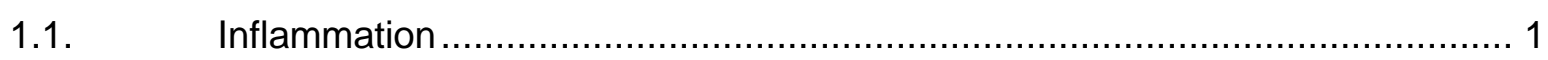

1.1.1. Mechanisms of inflammation .......................................................... 1

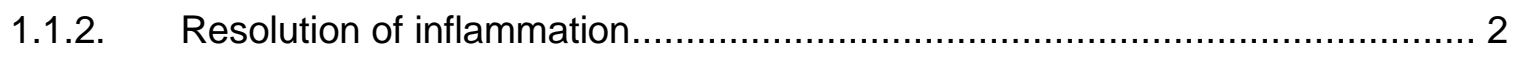

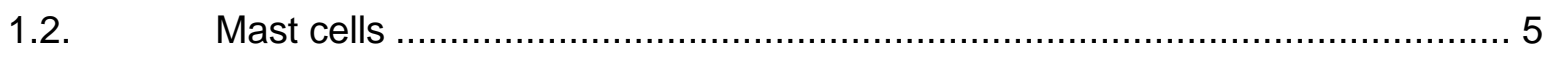

1.2.1. Morphology, Origin and Location of mast cells..................................... 5

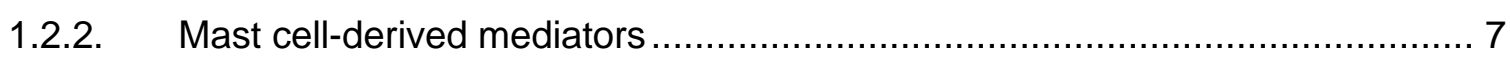

1.2.3. Mast cell-deficient mice models ........................................................... 8

1.3. Pattern Recognition Receptors ......................................................

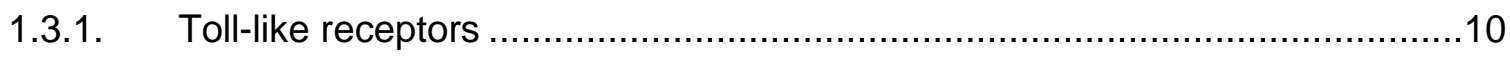

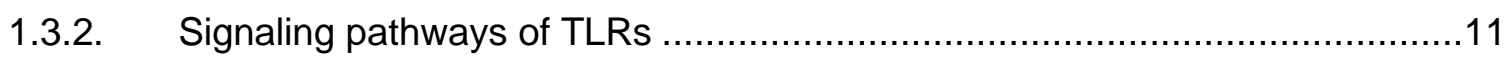

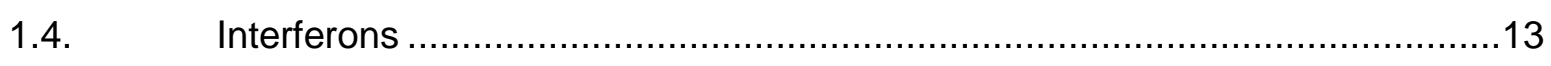

1.4.1. Induction and downstream signaling of type I IFNs ............................... 14

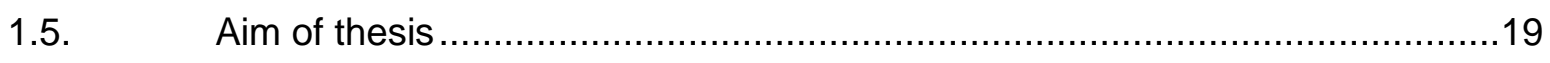

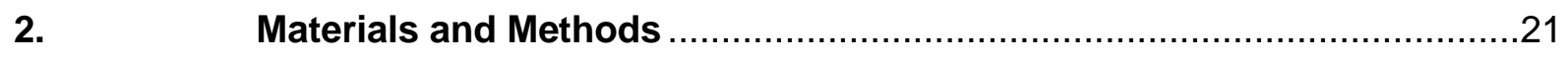

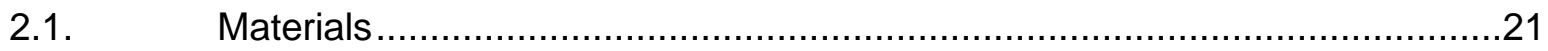

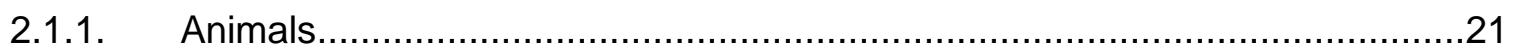

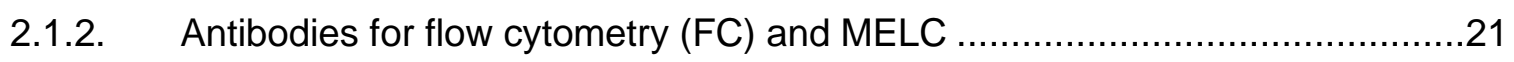

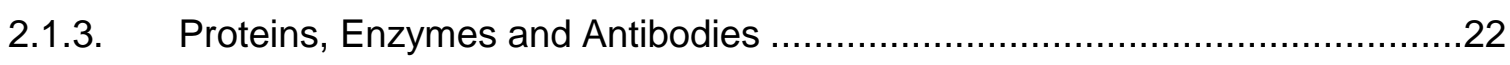

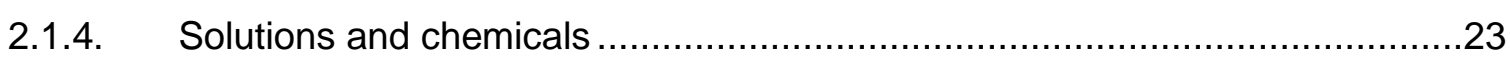

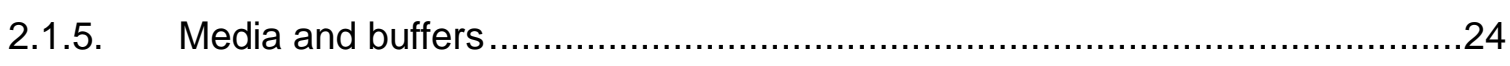

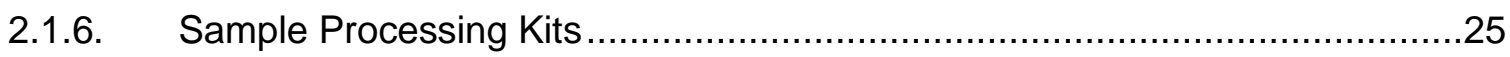

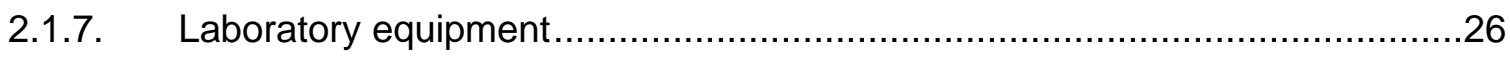

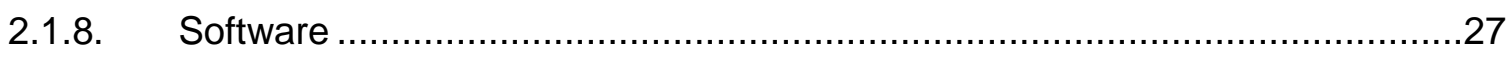

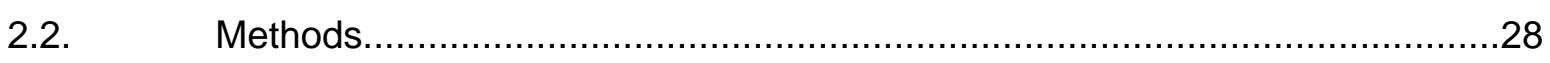

2.2.1. Mechanical nociception and paw edema measurement in mice.....................28

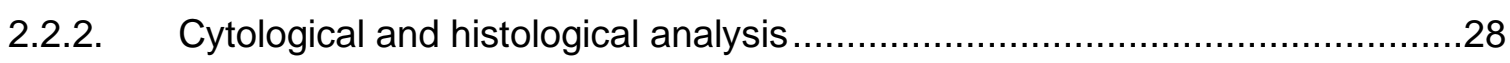

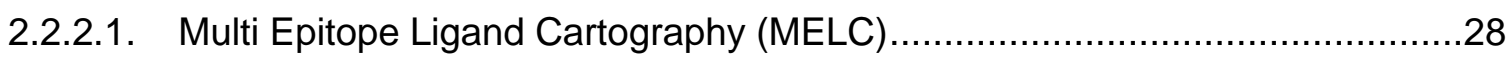

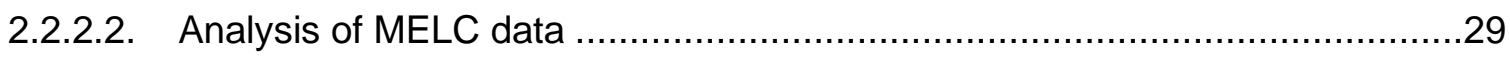

2.2.2.3. Polychromatic flow cytometry of immune cells in paw of mice ......................29

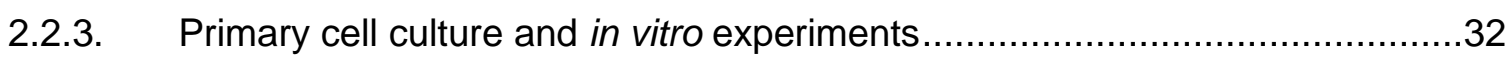


2.2.3.1. Isolation and cultivation bone marrow-derived mast cells (BMMC) …............32

2.2.3.2. Isolation and cultivation bone marrow-derived macrophages (BMDM) ...........33

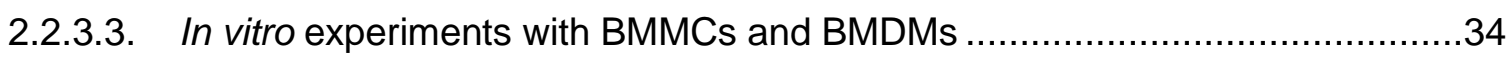

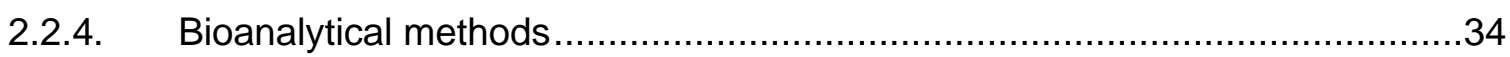

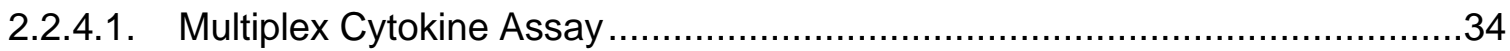

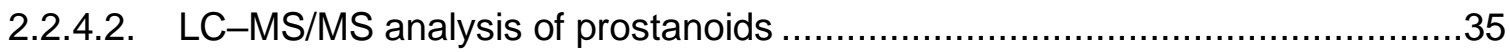

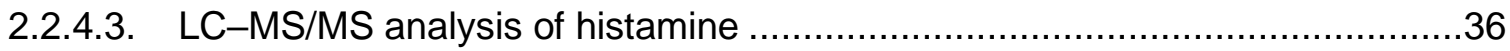

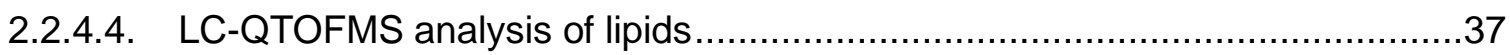

2.2.5. RNA Sequencing for differential expression analysis .................................38

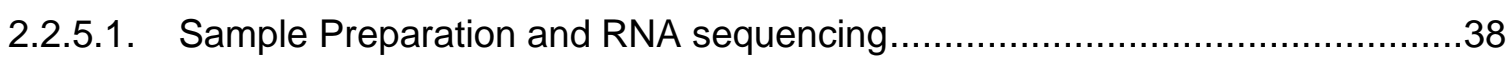

2.2.5.2. Analysis of RNA sequencing data............................................................... 40

3.

Results .45

3.1. Comparison of two locally induced inflammation models ............................45

3.1.1. Mechanical hypersensitivity induced by LPS and zymosan.........................45

3.1.2. Stronger local inflammation regarding edema formation and levels of recruited immune cells in zymosan model compared to LPS model ........................................46

3.1.3. Lipid profiles and chemokine levels indicate a systemic immune response to LPS and a local immune response to zymosan .................................................... 47

3.2. Role of mast cells during resolution of local inflammation ............................50

3.2.1. Mast cells reside in anti-inflammatory region during resolution phase of

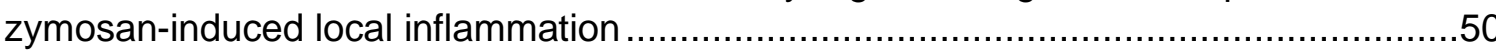

3.2.2. Mast cells influence macrophage phagocytosis and efferocytosis activity ......55

3.2.3. Screening of cytokines involved in inflammatory response after zymosaninjection in mast cell-deficient mice compared to control mice

3.3. Identification of mediators produced by mast cells ...................................64

3.3.1. Analysis of purity and maturity of bone marrow-derived mast cells ................64

3.3.2. Newly synthesized mediators released by BMMCs after TLR induction .........65

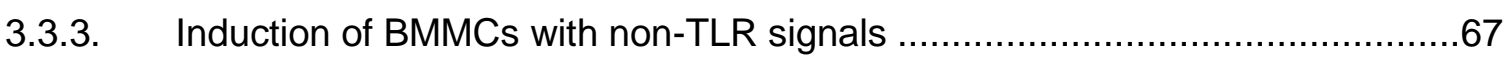

3.3.4. RNA-sequencing as untargeted approach to identify mediators produced by zymosan-stimulated mast cells reveals type I IFN response.......................................69

3.3.4.1. Quality assessment of samples, libraries and sequencing data ....................70

3.3.4.2. Differential expression analysis of RNA sequencing data shows differences between untreated and zymosan-treated samples

3.3.4.3. Gene ontology term analysis reveals zymosan-induced type I IFN response in mast cells 74

3.3.5. Validation of type I IFN response in mast cells........................................

3.3.5.1. Mast cells produce IFN- $\beta$ in vitro and in vivo...........................................

3.3.5.2. IFN- $\beta$ not required for release of IL-10 in mast cells .................................79

3.3.5.3. Release of IFN- $\beta$ by mast cells in response to zymosan and LPS requires internalization and phagosome maturation .... 
4.

Discussion

4.1. Comparison of two locally induced inflammation models ............................83

4.2. Anti-inflammatory microenvironment of mast cells .....................................84

4.3. Pro-resolving effect of resolution phase mast cells ....................................86

4.4. Phenotype of mast cells during resolution of inflammation ..........................88

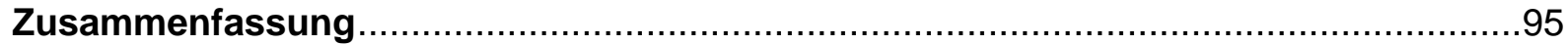

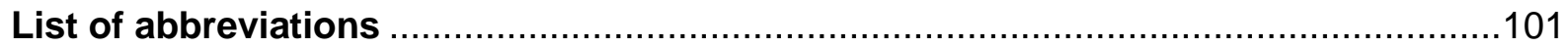

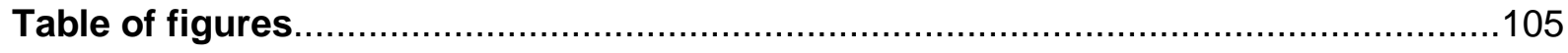

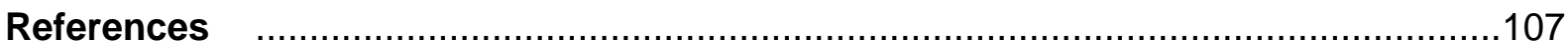

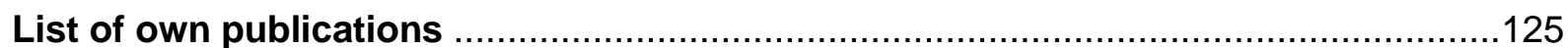




\section{Abstract}

Mast cells are long-lived tissue-resident leukocytes, located most abundantly in the skin and mucosal surfaces. They belong to the first line of defence of the body, protecting against invading pathogens, toxins and allergens. Their secretory granules are densely packed with a plethora of mediators, which can be released immediately upon activation of the cell. Next to their role in IgE-mediated allergic diseases and in promoting inflammation, potential antiinflammatory functions have been assigned to mast cells, depending on the biological setting. The aim of this thesis was to contribute to a better understanding of the role of mast cells during the resolution of a local inflammation. Therefore, in a first of step a suitable model of a local inflammation had to be identified. Since comparison of the two Toll-like receptor (TLR)-agonists zymosan and lipopolysaccharide (LPS), which are most commonly used to locally induce inflammation, revealed a systemic response after LPS-injection and a local inflammation after zymosan-injection, the TLR2 agonist zymosan was chosen for the subsequent experiments. Multi epitope ligand cartography (MELC) combined with statistical neighbourhood analysis showed that mast cells are located in an anti-inflammatory microenvironment next to M2 macrophages during resolution of inflammation, while neutrophils and M1 macrophages are located in the zymosan-filled core of the inflammation. Furthermore, infiltrating neutrophils during peak inflammation and an increasing population of macrophages phagocytosing neutrophils during resolution of inflammation could be observed. MELC as well as flow cytometry analysis of mast cell-deficient mice revealed a decreased phagocytosing activity of macrophages in the absence of mast cells. As an untargeted approach to identify mast cell-derived mediators induced by zymosan, mRNA sequencing of bone marrow-derived mast cells (BMMCs) was performed. Gene ontology term analysis of the sequencing data revealed the induction of the type I interferon (IFN) pathway as the dominant response. Contradicting previous studies, I could validate the production of IFN- $\beta$ by mast cells in response to zymosan and LPS in vitro. Furthermore IFN$\beta$ expression by mast cells was also detected in vivo. In accordance with previous studies regarding other cell types the release of IFN- $\beta$ by mast cells depends on endosomal signaling. The potential of IFN- $\beta$ to enhance the phagocytosing activity of macrophages has been demonstrated recently. Besides IFN- $\beta$, various other mediators with reported enhancing effects on macrophage phagocytosis were also induced by zymosan in BMMCs, including Interleukin (IL)-1 $\beta$, IL-4, IL-13, and Prostaglandin (PG) $E_{2}$. Thus, either one of these mediators alone or a combination of them could promote macrophage phagocytosis.

In conclusion, I herein present mast cells as a novel source for IFN- $\beta$ induced by non-viral TLR ligands and demonstrate their enhancing effect on macrophage phagocytosis, thereby contributing to the resolution of inflammation. 


\section{Introduction}

1.1. Inflammation

The term inflammation is used to describe the response of the immune system towards injury or infection, to protect the body against pathogens, toxic compounds and damaged cells. Acute inflammation can be accompanied by five typical symptoms, namely rubor (redness), calor (heat), tumor (swelling), dolor (pain), and function leasa (loss of function). Rubor, calor, tumor and dolor have been described already in the first century AD by Cornelius Celsus, while the first mentioning of the fifth symptom, function leasa (loss of function), probably originated from Rudolph Virchow in 1989. While an acute inflammatory response is in general protective, removing harmful stimuli and repairing injured tissue, a failure in resolution of inflammatory processes can lead to chronic inflammation and cause immense harm.

\subsubsection{Mechanisms of inflammation}

The inflammatory response is initiated upon detection of harmful stimuli like pathogenassociated molecular patterns (PAMPs) or danger-associated molecular patterns (DAMPs). They are recognized by germline-encoded pattern recognition receptors (PRRs), which are present on immune cells as well as non-immune cells (Akira et al., 2006; Kumar et al., 2009; Takeuchi \& Akira, 2010).

Signaling through PRRs leads to the activation of inflammatory pathways, most commonly the nuclear factor kappa-B (NF-kB), the mitogen-activated protein kinase (MAPK), and the Janus kinase-signal transducer and activator of transcription (JAK-STAT) pathways. Activation of these pathways results in the production and release of various inflammatory mediators, including cytokines, chemokines, enzymes, reactive oxygen species (ROS) and eicosanoids, mostly by tissue resident macrophages and mast cells. The first mediators released include vasoactive mediators such as histamine, serotonin, and prostanoids, and chemoattractants such as CXCL1, CXCL2, and CCL1, also referred to as monocyte chemoattractant protein-1 (MCP-1) (Beck-Schimmer et al., 2005; De Filippo et al., 2008). They promote vascular dilation, enhanced permeability of capillaries, and the recruitment of leukocytes and plasma to the site of inflammation, leading to redness, swelling and heat of the affected tissue. The symptom pain meanwhile arises due to the increased sensitivity of 
sensory neurons to noxious and innocuous stimuli, which is described by the terms hyperalgesia and allodynia, respectively (Ronchetti et al., 2017). This increased sensitivity is induced by inflammatory mediators like histamine, bradykinin, prostaglandins and others (Dray, 1995; Moalem \& Tracey, 2006). A combination of factors may also lead to loss of function, such as difficulty to breathe during a respiratory infection or impaired movement of an inflamed joint due to swelling and pain (Chandrasoma \& Taylor, 1997).

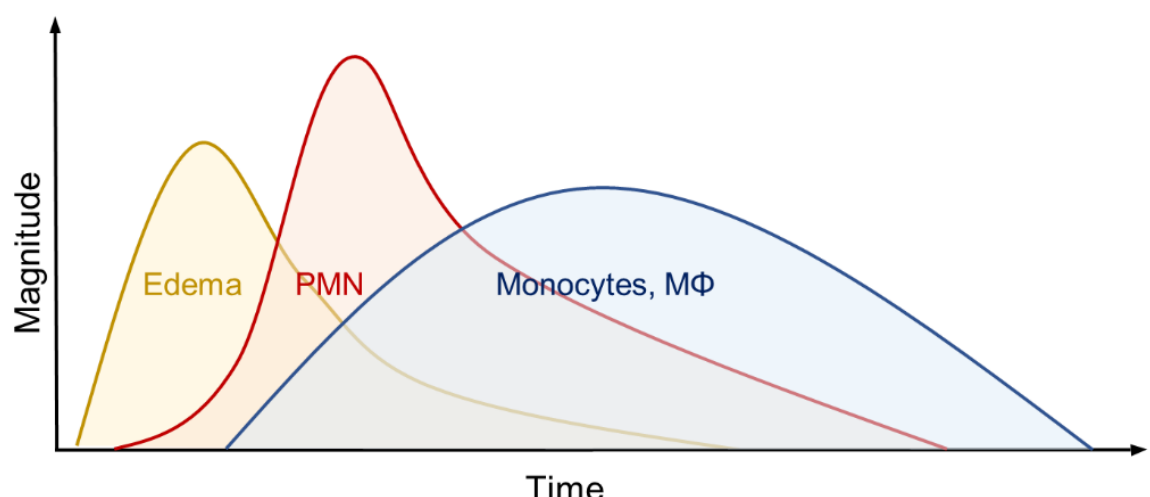

Figure 1: The acute inflammatory response. Figure adapted from Sansbury \& Spite (2016). The onset of a local acute inflammation is characterized by edema formation and infiltration of polymorphnucelar neutrophils (PMNs), followed by the recruitment of monocytes. PMNs engulf and degrade pathogens and then undergo apoptosis. Apoptotic cells are then cleared by monocyte-derived macrophages (MФ) by efferocytosis. Further PMN recruitment is limited by pro-resolving mediators, injured tissue is repaired and homeostasis is reestablished.

Following the rapid influx of neutrophils to the inflamed area, monocytes are recruited to the tissue where they mature into macrophages (Figure 1) (Fujiwara \& Kobayashi, 2005; Italiani \& Boraschi, 2014). Pro-inflammatory macrophages prolong the survival of neutrophils by releasing a variety of growth factors such as granulocyte-macrophage colony-stimulating factor (GM-CSF), granulocyte colony-stimulating factor (G-CSF), and tumor necrosis factor alpha (TNF- $\alpha$ ) (Prame Kumar et al., 2018; Takano et al., 2009). Both macrophages and neutrophils release mediators to kill and degrade pathogens and remove them by phagocytosis (Silva, 2010). Once harmful stimuli are removed, neutrophils undergo apoptosis and are removed by macrophages (Greenlee-Wacker, 2016; Ortega-Gómez et al., 2013). Finally, healing of the tissue takes place (Bennett et al., 2018).

\subsubsection{Resolution of inflammation}

The physiological role of inflammation is the protection of the body from infection and injury. A successful acute inflammation eliminates noxious stimuli and leads to healing of damaged 
tissue, resulting in the return to homeostasis. However, inflammation can develop into a pathological process when it becomes persistent.

Numerous diseases are associated with chronic inflammation, e.g. rheumatoid arthritis (Schett, 2019), psoriasis (Benezeder \& Wolf, 2019), asthma (Finotto, 2019), multiple sclerosis (Ruiz et al., 2019), Crohn's disease (Schmitt et al., 2019), ulcerative colitis (Neurath \& Leppkes, 2019), atherosclerosis (Carracedo et al., 2019) and even cancer (Coussens \& Werb, 2002), obesity (H. Lee et al., 2013), diabetes (Tsalamandris et al., 2019) and mental health disorders (Peirce \& Alviña, 2019). Those diseases are common in Western societies and are a burden for the patients as well as the healthcare system. Chronic inflammatory diseases can lead to irreversible and progressive tissue destruction, such as in rheumatoid arthritis, ulcerative colitis, and asthma (Boldeanu et al., 2014; Carroll et al., 1993; Mclnnes \& Schett, 2017). Patients with inflammatory bowel diseases are furthermore more likely to develop cancer, and tumors in cancer patients are affected by their inflammatory microenvironment (Kiesslich et al., 2003; Landskron et al., 2014; Terzić et al., 2010).
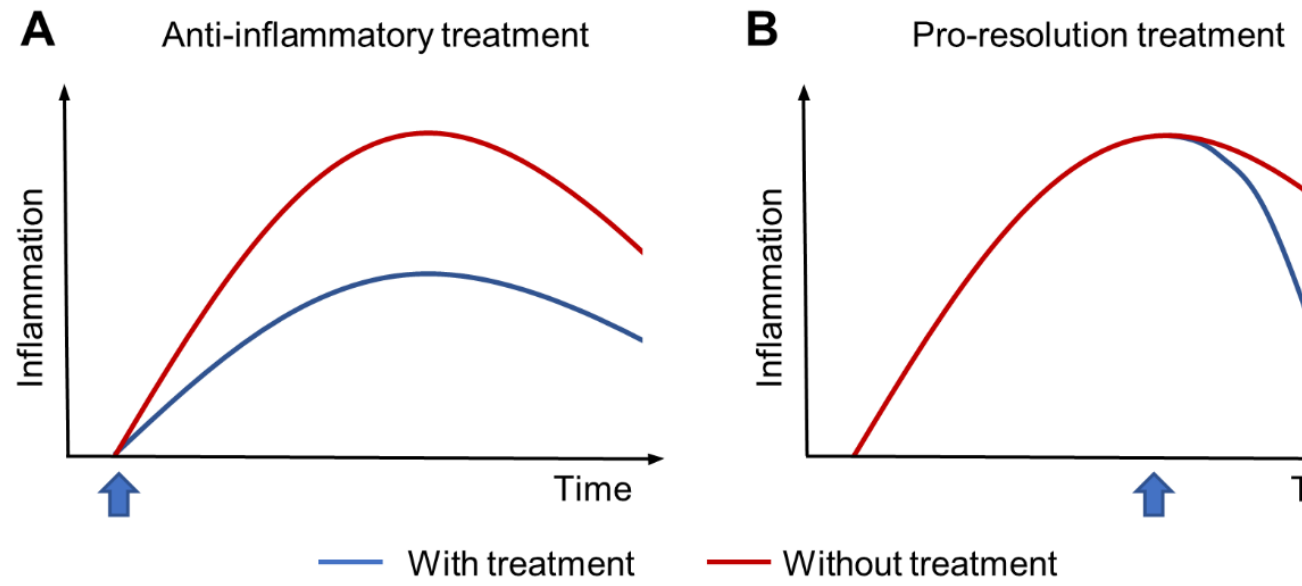

Figure 2: Anti-inflammatory versus pro-resolution treatment of inflammation. Figure adapted from Fullerton \& Gilroy (2016). (A) Anti-inflammtory treatment dampens inflammation from the onset by inhibiting proinflammatory mediators without necessarily accelerating the resolution. (B) Pro-resolution treatment accelerates the resolution without necessarily affecting the onset of inflammation. Blue arrows indicate the beginning of the treatment.

Many of those chronic inflammatory diseases are thought to be induced by tissue stress or malfunction, which evoke an immune response referred to as para-inflammation, in contrast to an acute inflammation induced by infection or tissue injury (Medzhitov, 2008). An example for a chronic inflammatory state without previous infection or injury is adipose tissue in obesity (Hotamisligil, 2017). Exceeding of the fat storage capacity of adipocytes leads to a stress response, resulting in the production of cytokines (Hotamisligil, 2006). These stressinduced para-inflammatory mechanisms share the common feature of a disturbed homeostasis with injury- or infection-induced inflammation. 
While processes that promote or hinder the resolution of inflammation may be specific for a disease or affected tissue, understanding universal principles of resolution mechanisms may be instrumental to help overcome the pathomechanisms of chronic inflammation and develop strategies to initiate resolution. So far, chronic inflammatory diseases are routinely treated by suppressing pro-inflammatory effector pathways by targeting cytokines or other mediators involved, often by using non-steroidal anti-inflammatory drugs (NSAIDs) and biologicals such as TNF- $\alpha$ blocking antibodies (Fullerton \& Gilroy, 2016). By inhibiting the factors that drive the inflammation, the inflammation is dampened, but not resolved (Figure 2); therefore, treatments usually have to be given live-long. Furthermore, physiological immune responses are partly suppressed, leading to an increased infection risk.

Natural resolution mechanisms are initiated shortly after the onset of inflammation. Neutrophils are the first leukocytes to be recruited to the site of inflammation and they are also the most abundant ones. They are essential to remove noxious stimuli from the tissue, which is a prerequisite for initiation of resolution. An exaggerated influx of neutrophils however can be detrimental and their recruitment has to be stopped in order to return to tissue homeostasis. Furthermore, vascular permeability has to return to a normal level and apoptotic neutrophils present in the tissue have to be removed. They express "find me" and "eat me" signals, which triggers macrophages to engulf them. The uptake of apoptotic cells by macrophages is called efferocytosis and has a profound effect on resolution of inflammation, amongst others by preventing the release of intracellular components by dying cells that could contribute to prolonged inflammation and lead to autoimmunity (Kourtzelis et al., 2020; Maderna \& Godson, 2003; Savill et al., 2002). Another important hallmark of the resolution of inflammation is the reprogramming of macrophages from classically activated pro-inflammatory macrophages, also referred to as M1 macrophages, to alternatively activated anti-inflammatory, pro-resolving macrophages, referred to as M2 macrophages. While M1 macrophages produce pro-inflammatory cytokines and contribute to elimination of pathogens, M2 macrophages release pro-resolving mediators and clear apoptotic cells (Viola et al., 2019). Finally, to return to homeostasis and prevent fibrosis or scar formation and thereby loss of function of the tissue after inflammation, repair of the inflammation-induced injury is required; this process is orchestrated by macrophages (Lech \& Anders, 2013).

On a molecular level, resolution is based on a network of lipid and protein mediators. Antiinflammatory and immune-suppressive cytokines, such as transforming growth factor beta (TGF- $\beta$ ) and interleukin (IL)-10, are able to inhibit the production of pro-inflammatory mediators (Conaway et al., 2017; Yoshimura \& Muto, 2010). Furthermore, IL-10 and TGF- $\beta$ have been reported to induce the $\mathrm{M} 2$ phenotype in macrophages, among other mediators like IL-4, IL-13 and TLR agonists (Benoit et al., 2008; Martinez et al., 2009). Resolution of inflammation is also accompanied by a class switch of lipid mediators (B. D. Levy et al., 
2001). After the initial production of mediators activating and amplifying the cardinal signs of inflammation, like prostaglandins (PGs) and leukotriens (LTs), an active switch to the production of pro-resolving mediators takes place. PGs and LTs are derived from endogenous arachidonic acid released from membrane phospholipids, mediated by cyclooxygenase (COX) and lipoxygenase (LOX) pathways, respectively. A third class of arachidonic acid-derivates comprises the pro-resolving lipoxins ( $L X s)$, such as $L X A_{4}$. Synthesis of $\mathrm{LXA}_{4}$ is mediated by two distinct LOXs present in interacting cells, such as neutrophils, eosinophils or macrophages, interacting with platelets or endothelial, epithelial or parenchymal cells (Serhan et al., 1984). Other pro-resolving lipid mediators, namely resolvins, protectins, and maresins, are derived from exogenous, dietary fatty acids, such as $\omega-3$ polyunsaturated fatty acids (PUFAs) found in fish oil (Serhan et al., 2008). LXs, resolvins, protectins and maresins have various anti-inflammatory and pro-resolving functions, including limiting the recruitment of neutrophils, stimulating the recruitment of monocytes, as well as stimulating efferocytosis (Serhan et al., 2008).

In summary, an orchestrated immune response and interplay between the cell types involved is necessary to successfully resolve inflammation and prevent progressive tissue damage.

\subsection{Mast cells}

Mast cells are cells of the immune system that contain many secretory granules filled with pre-formed bioactive compounds. They can be found in almost all vascularized tissues, close to surfaces exposed to the environment like the skin, gastrointestinal tract and respiratory epithelium, where they are one of the first cells to encounter harmful environmental stimuli like pathogens, allergens, and toxins. Their location close to potential entry sites of pathogens together with their ability to rapidly release a massive number of ready-made mediators enables them to be part of the first line of defence of the immune system. There is rising evidence that mast cells are not only key initiators of inflammation, but that they also have the potential to act immunomodulatory to the extent of resolving the immune response, depending on the biological setting (Galli et al., 2008).

\subsubsection{Morphology, Origin and Location of mast cells}

Rodent mast cells originate from hematopoietic precursors in the bone marrow of the common myeloid progenitor (CMP) lineage. After commitment of the precursors to the mast cell lineage, they enter the blood circulation as mast cell progenitors (MCp) and migrate to 
their target tissue (Dahlin \& Hallgren, 2015). Those MCps are small and barely have any granules before developing into mature mast cells. Differentiation of MCps takes place under the influence of stem-cell factor (SCF, also known as Kit ligand), IL-3 and other distinct growth factors within the peripheral tissue (Rennick et al., 1995).

Signaling of SCF and its receptor c-Kit is crucial for the development of murine mast cells and is also an essential growth factor for human mast cells. SCF exists in a soluble form and in a membrane bound form and is constitutively expressed by endothelial cells, fibroblasts and stromal cells (Galli et al., 1993; Heinrich et al., 1993). Beyond its function in the maturation of mast cells, SCF is furthermore a critical chemotactic factor for the recruitment of MCps to target tissues. Additionally, SCF stimulates the proliferation of mast cell progenitors and promotes the survival of mast cells. The development, survival and migration of mast cells is reviewed in detail by Okayama and Kawakami (2006).

The first mention of mast cells was probably in 1863, when Friedrich von Recklinghausen described granulated cells preferentially located close to blood vessels. Paul Ehrlich later coined the name "Mastzellen", after discovering a population of cells filled with granules with the capacity to metachromatically stain with aniline dyes like toluidine blue (Ehrlich, 1878). In 1966, Lennart Enerbäck described two subpopulations of rodent mast cells differing in staining characteristics and tissue homing: connective tissue mast cells (CTMCs) and mucosal mast cells (MMCs). CTMCs contain heparin and are therefore stained red by safranin, while MMCs lack heparin. These two phenotypically distinct subpopulations furthermore differ in morphology, mediator content and T-cell dependency. MMCs are smaller than CTMCs, with 5 - $10 \mu \mathrm{m}$ compared to $10-20 \mu \mathrm{m}$ in diameter, and while CTMCs contain histamine and serotonin, MMCs lack serotonin (Elieh Ali Komi et al., 2020). Subtypes of human mast cells are distinguished by their protease composition as tryptase-only $\left(\mathrm{MC}_{\mathrm{T}}\right)$, chymase-only $\left(\mathrm{MC}_{\mathrm{C}}\right.$ ) or tryptase- and chymase-positive (MC $\mathrm{TC}$ ) (Irani \& Schwartz, 1994). In contrast to rodents, humans mostly have mixed populations of mast cell subtypes in tissues (Welle, 1997).

Mast cells can be distinguished from other cells by their expression of both the receptors cKit (also known as CD117) and FcERI. FceRI is a high-affinity receptor for the Fc region of immunoglobulin ( $\mathrm{Ig}) \mathrm{E}$ and is involved in $\mathrm{IgE}$-mediated allergic reactions. IgE molecules are bound irreversibly to FcERI, resulting in the mast cell being coated with IgE. Antigen binding to $\lg E$ on the surface of a mast cell then leads to degranulation and release of histamine and other vasoactive mediators (Galli \& Tsai, 2012). Mast cells have been mostly recognized for their role in the pathophysiology of IgE-mediated allergic responses, but they are also involved in the regulation of pleiotropic physiological functions, including vasodilation and angiogenesis, elimination of pathogens and parasites, as well as regulating functions of other 
immune cells. The physiological as well as the pathological actions of mast cells depend on the biological activities of the mediators produced and released by them.

\subsubsection{Mast cell-derived mediators}

Mast cells represent a potential source of a variety of mediators with a wide range of possible functions, including antimicrobial activity, recruitment and activation of other immune cells, effects on epithelial cells and degradation of endogenous toxic mediators (Tsai et al., 2011). The granules of mast cells are filled with preformed mediators that can be released directly upon activation of the cell by various external stimuli (Moon et al., 2014). In addition to the release of preformed mediators, mast cell activation also leads to de novo synthesis of lipid mediators, cytokines and chemokines. The most well-known preformed compound stored in granules of mast cells is probably histamine, which is present in all subtypes of mast cells in all species. Histamine causes vasodilation and increases vascular permeability and is best known for its role in allergic reactions (Ashina et al., 2015).

Other preformed mediators next to histamine include proteases, proteoglycans, lysosomal enzymes and the cytokine TNF- $\alpha$. About $25 \%$ of the proteins in mast cells are proteases, three of them being specific for mast cells, namely chymase, tryptase and carboxypeptidase A3 (Pejler et al., 2007). The proteases are stored in their active form, meaning they can carry out their enzymatic activity directly upon release. Substrates cleaved by mast cell proteases include a variety of inflammatory mediators, which can be either activated or inactivated by cleavage (Dai \& Korthuis, 2011). Proteoglycans like heparin are required for storage and functionality of proteases and biogenic amines and are responsible for the characteristic staining of mast cells by toluidine blue (Ribatti, 2018). The mostly pre-formed cytokine TNF- $\alpha$ has been shown to initiate and amplify an inflammatory response by contributing to leukocyte recruitment and is therefore regarded as a pro-inflammatory cytokine (Biedermann et al., 2000; J. R. Gordon \& Galli, 1990). The release of preformed mediators takes place within seconds after activation (Moon et al., 2014).

In a next step, only minutes after activation of the mast cell, lipid mediators can be released, since no transcription is needed. Eicosanoids are the most prominent lipid mediators produced by mast cells. The eicosanoids in mast cells include PGs produced by COX-1 and COX-2, hydroperoxy-leicosatetraenoic acids (HPETEs) and LTs generated by LOXs, epoxyeicosatrienoic acids (EETs) and hydroxyeicosatetraenoic acids (HETEs) produced by cytochrome P450 enzymes. One of the main eicosanoids produced by mast cells is $\mathrm{PGD}_{2}$ (Boyce, 2007). Another mast cell derived eicosanoid is thromboxane $A_{2}\left(T X A_{2}\right)$ (Fonteh et al., 1995; Mita et al., 1999), which has been shown to be involved in edema formation and 
mechanical hypersensitivity in capsaicin-induced neurogenic inflammation (Tarighi et al., 2019).

Since de novo synthesis of cytokines, chemokines and growth factors requires induction of gene transcription and translation, it takes hours for the proteins to be released after activation of the mast cell. De novo synthesized mediators include IL-1 $\beta$, IL-2, IL-3, IL-4, IL-5, IL-6, IL-9, IL-10, IL-11, IL-12, IL-13, IL-16, IL-33, epidermal growth factor (EGF), fibroblast growth factor (FGF), GM-CSF, interferon (IFN) - $\gamma$, nerve growth factor (NGF), platelet-derived growth factor (PDGF), SCF, TGF- $\beta$ and vascular endothelial growth factor (VEGF) (Theoharides et al., 2012). Mast cell-derived cytokines have various functions, such as activation of neighbouring cells and recruitment of immune cells (Mukai et al., 2018).

The subset of mediators released by a mast cell depends on the stimuli activating the cell, with different stimuli leading to different responses. This is possible due to various receptors signaling through diverse pathways and to the selective release of granules and other compartments, such as vesicles and exosomes. While all granules of a mast cell are released at once in anaphylactic degranulation, mast cells are also able to release only subsets of the heterogenous granules by piecemeal degranulation (Moon et al., 2014; Wernersson \& Pejler, 2014). Furthermore, mast cells are able to recover from degranulation, form new granules and reside in the tissue for months, making them rather long-lived cells (Hammel et al., 1989; Padawer, 1974; Walker, 1961).

\subsubsection{Mast cell-deficient mice models}

Besides their well described role as effector cells in allergic reactions, mast cells are considered to have a wide range of functions in innate and adaptive immunity. The broad range of mast cell products discovered in vitro potentially enables mast cells to contribute to detrimental as well as protective processes during all stages of inflammation. To investigate the actual role of mast cells and their mediators in vivo, suitable models are necessary.

To study the contribution of mast cells to biological processes, both fully mast cell-deficient mice and mice deficient of specific mast cell populations in tissues of interest can be of use, while the deletion or blocking of mast cell products can help to identify the means by which mast cells influence their environment in particular biological settings. To ablate mast cells in vivo, various genetic approaches have been addressed (reviewed in Reber, Marichal, and Galli 2012; Rodewald and Feyerabend 2012). In older models, mast cell ablation was achieved by defects of the receptor Kit, which is encoded on the mouse $\mathrm{W}$ locus and is required by mast cells for growth and survival. The mutant mice $\mathrm{Kit}^{\mathrm{W} / \mathrm{Wv}}$ and $\mathrm{Kit}^{\mathrm{W}-\mathrm{sh} / \mathrm{W} \text {-sh }}$ are the 
two most commonly used Kit mutant models. The protein encoded by $\mathrm{Kit}^{\mathrm{W}}$ is truncuated and is therefore not expressed on the cells surface (Hayashi et al., 1991), while the Kit ${ }^{\mathrm{Wv}}$ encoded protein has a reduced kinase activity due to a mutation in the Kit tyrosine kinase domain (Nocka et al., 1990). In the $\mathrm{Kit}^{\mathrm{W} \text {-sh }}$ allele, there is a large inversion in the transcriptional regulatory element upstream of the Kit locus, affecting the expression of Kit (Berrozpe et al., 2006; Nigrovic et al., 2008).

Unfortunately, Kit mutant mice have several phenotypic abnormalities besides their mast celldeficiency, since Kit is widely expressed in cells both within and outside of the immune system (Reber et al., 2012). The high expression of Kit on hematopoietic stem and progenitor cells is lost with differentiation in all hematopoietic cells except for mast cells (Galli et al., 1993). The defects in the hematopoietic system of Kit mutant mice are distinct but partly overlapping between the different mutant mouse strains and include anaemia and either reduced or increased number of neutrophils, basophils, megakaryocytes and other immune cells. Furthermore $\mathrm{Kit}^{\mathrm{W} / \mathrm{Wv}}$ mice are sterile, while $\mathrm{Kit}^{\mathrm{W} \text {-sh/W-sh }}$ mice are fertile, which is one of the reasons why they have been preferred over $\mathrm{Kit}^{\mathrm{W} / \mathrm{Wv}}$ mice. Especially the effect of Kit mutations on cells involved in innate and adaptive immune responses apart from mast cells obviously has the potential to interfere with the outcome of experiments meant to study the role of mast cells. One approach to overcome the problems of Kit mutant mice was to reconstitute them with mast cells, for example by injecting them intravenously, intraperitonally or intradermally. This was meant to rule out the influence of cells other than mast cells on the outcome of the experiment.

Intriguingly, there have been discrepancies in several cases between the outcome of experiments using Kit mutants and mast cell-reconstituted Kit mutants compared to newer Kit-independent mast cell-deficient mice and normal mice (Rodewald \& Feyerabend, 2012). Reconstitution with mast cells might result in mast cell populations different from endogenously developed ones, regarding their distribution and behaviour, leading to compensation not only of the mast cell deficiency but also other Kit-derived abnormalities absent in wild type mice. While the exact mechanisms behind this still remain to be understood, it is clearly favourable to use mast cell-deficiencies that are independent of mutations in the pleiotropic Kit. To overcome potential difficulties in interpreting results based on Kit mutant mice, several groups have developed Kit-independent mast cell-deficient mice. The common feature of all these mouse strains is the expression of Cre recombinase under the control of mast cell-specific or -associated promoters (Dudeck et al., 2011; Feyerabend et al., 2011; Lilla et al., 2011; Otsuka et al., 2011).

The mouse strain used in this thesis is Mcpt5-Cre DTA developed by Dudeck et al. (Dudeck et al., 2011). They generated constitutive mast cell deficiency by cross-breeding R-DTA mice 
(Voehringer et al., 2008) to the mast cell-specific Cre transgenic line Mcpt5-Cre (Scholten et al., 2008). In this mouse strain, the Cre recombinase is expressed under the control of the mast cell protease (Mcpt) 5 promoter; therefore, the expression of Cre is restricted to mast cells. Thereby the loxP flanked stop cassette controlling the expression of diphtheria toxin $A$ (DTA) is excised by Cre exclusively in mast cells, leading to their death due to production of the toxin followed by inhibition of protein synthesis in the cells. Since Mcpt5 is only expressed in CTMCs, only CTMCs are depleted while MMCs are unaffected. Peritoneal mast cells are reduced by $98 \%$, skin mast cells $89 \%-97 \%$, varying between different locations in the body.

\subsection{Pattern Recognition Receptors}

Toxic compounds, invading pathogens or damaged cells evoke the immune response of the body as a defence system against harmful stimuli. The initial detection of harmful stimuli is mediated by innate pattern recognition receptors (PRRs), recognizing exogenous pathogenassociated molecular patterns (PAMPs) and endogenous damage-associated molecular patterns (DAMPs). PRRs in mammals include Toll-like receptors (TLRs), RIG-I-like receptors (RLRs), Nod-like receptors (NLRs), AIM2-like receptors (ALRs), C-type lectin receptors (CLRs), and intracellular DNA sensors (Akira et al., 2006; Cai et al., 2014). The best characterized PRRs are TLRs (Mogensen, 2009).

\subsubsection{Toll-like receptors}

There are 13 known TLRs in mammals, named TLR1-13. TLR1-10 are found in humans and TLR1-9 and 11-13 are found in mice. Common features shared by all TLR family members are a leucine-rich repeat (LRR) domain in their extracytoplasmic domain mediating PAMP recognition and an intracytoplasmic Toll-interleukin-1 receptor (TIR) domain initiating signal transduction (Botos et al., 2011). All TLRs are transmembrane proteins; TLR1, 2, 4, 5 and 6 are found predominantly on the cell surface, while TLR3, 7, 8, 9, 11, 12 and 13 are located in endosomal compartments. Intracellular TLRs are mostly known to recognize bacterial and viral nucleic acids, while cell surface located TLRs recognize microbial cell wall and cell membrane components in the extracellular space. Each TLR recognizes a subset of PAMPs or DAMPs that is distinct from or overlapping with the subset recognized by other TLRs. Most TLRs are known to interact with their ligands as homodimers, while TLR2 forms heterodimers with TLR1 or TLR6 (Oliveira-Nascimento et al., 2012). 
TLR2 and TLR4 are both widely expressed and recognize an impressive variety of pathogens and are therefore among the best studied PRRs (Mukherjee et al., 2016). The spectrum of microbial components recognized by TLR2 includes diverse bacterial glycoproteins and zymosan, a fungal cell wall component (lkeda et al., 2008; Takeda et al., 2003). TLR10 seems to recognize bacterial and fungal cell surface components in cooperation with TLR2, but downstream signaling and function of this receptor has not been identified yet (Guan et al., 2010). Dectin1, a receptor recognizing $\beta$-glucan from fungal cell walls, has also been shown to collaborate with TLR2 (Gantner et al., 2003). TLR4 is mostly known for its essential role in recognition of gram-negative bacteria derived lipopolysaccharides (LPS) (Hoshino et al., 1999; Poltorak et al., 1998), but has also been shown to recognize several heat shock proteins of exogenous and endogenous origin and other self-derived DAMPs (Molteni et al., 2016).

\subsubsection{Signaling pathways of TLRs}

Upon binding of a ligand to the LRR domain of the TLR, the receptor dimer is stabilized and adaptor molecules are recruited to the TIR domain of the TLR. The five known adaptor molecules in mammals are Myeloid differentiation primary-response protein 88 (MyD88), TIR domain-containing adaptor protein (TIRAP, by some also referred to as MyD88-adaptor-like (MAL)), TIR domain containing adaptor protein inducing IFN- $\beta$ (TRIF), TRIF related adaptor molecule (TRAM) and Sterile $\alpha$ - and armadillo-motif-containing protein (SARM) (Akira \& Takeda, 2004). Two distinct signaling pathways can take place after TLR activation; the MyD88-dependent signaling pathway and the MyD88-independent signaling pathway, also referred to as the TRIF-dependent signaling pathway. The adaptor molecule MyD88 is used by all TLRs, except for TLR3, while the TRIF-dependent pathway is specific for TLR3 and TLR4 (Kawasaki \& Kawai, 2014).

In the MyD88-dependent signaling pathway of TLR2 and TLR4, both MyD88 and TIRAP bind to the activated receptor dimer by TIR-TIR interactions (Figure 3). Then, members of the interleukin-1 receptor associated kinase (IRAK) kinase family are recruited, forming a complex with MyD88 referred to as Myddosom, leading to the phosphorylation and dissociation of IRAKs. IRAKs then activate TRAF6, which in turn enhances the activity of the $I_{\kappa} B$ kinase $(I K K)$. The activated IKK then degrades the inhibitor of NF- $\kappa B\left(I_{\kappa} B\right)$, enabling NF$\kappa B$ to translocate to the nucleus, resulting in the induction of inflammatory genes. In parallel TRAF6 activates the MAPK pathway, resulting in the activation of AP-1, another transcription factor upregulating cytokine gene expression. 
The MyD88-independent, TRIF-dependent pathways utilized by TLR3 and TLR4 differ from each other. TLR3 directly recruits TRIF upon ligand binding, while TLR4 recruits TRIF via TRAM. TRIF then recruits TRAF3, leading to the phosphorylation and thereby activation of IRF3. IRF3 then forms dimers either with another TRAF3 or with TRAF7 and translocates to the nucleus, resulting in the transcription of IFN- $\beta$ and IFN-inducible genes. In the case of TLR4, induction of type I IFNs is mediated by TRAF3 homodimers, while TRAF7 is required for TLR3-mediated induction of type I IFNs (Honda, Yanai, Takaoka, et al., 2005). Next to type I IFN induction, TRIF activates NF- $\mathrm{B}$ via TRAF6, upregulating the transcription of inflammatory genes.

The type I IFN pathway can also be induced in a MyD88-dependent way by TLR7-9, which lack the TRIF-dependent signaling pathway (Kawai et al., 2004; Takeda \& Akira, 2005). It has been demonstrated that only interferon regulatory factor (IRF) 7, but not IRF3, is activated by MyD88 and interacts with TRAF6 upon TLR7-9 induction and that IRF7 is required for type I IFN induction (Honda et al., 2004; Honda, Ohba, et al., 2005; Honda, Yanai, Negishi, et al., 2005). Meanwhile, the activation of NF- $\kappa B$ and the subsequent induction of pro-inflammatory cytokines is independent of IRF7. The induction of type I IFNs by cell surface located TLRs other than by TLR4 has long been thought to be impossible due to their lack of TRIF-dependent signaling and MyD88 only interacting with IRF7 in endosomal compartments. By now it has been discovered that TRL2 can also induce the type I IFN response, but only upon receptor internalization (Barbalat et al., 2009; Bauernfeind \& Hornung, 2009). The endosomal MyD88-dependent IFN induction has been shown to require IRF7, just like in the MyD88-dependent IFN induction by endosomal TLR9. Furthermore, it has been demonstrated that TLR4 induction of the type I IFN response also only takes place in endosomal compartments (Kagan et al., 2008). Upon ligand binding to the initially cell surface located TLR4, MyD88 is recruited and leads to the induction of inflammatory cytokines. Then, TLR4 is internalized into Rab5-positive endosomes, recruits the adaptors TRAM and TRIF, and induces the type I IFN response (Figure 3).

In conclusion, the induction of different pathways by TLRs takes place in a spatio-temporally dependent way and the common denominator of TLR-induced type I IFN signaling seems to be the dependence on the subcellular localization of the receptor (Perkins \& Vogel, 2015). Furthermore, the recruitment of different subsets of adaptor molecules might be one explanation for the ability of TLRs to differentially induce overlapping but distinct pathways in response to different ligands. 


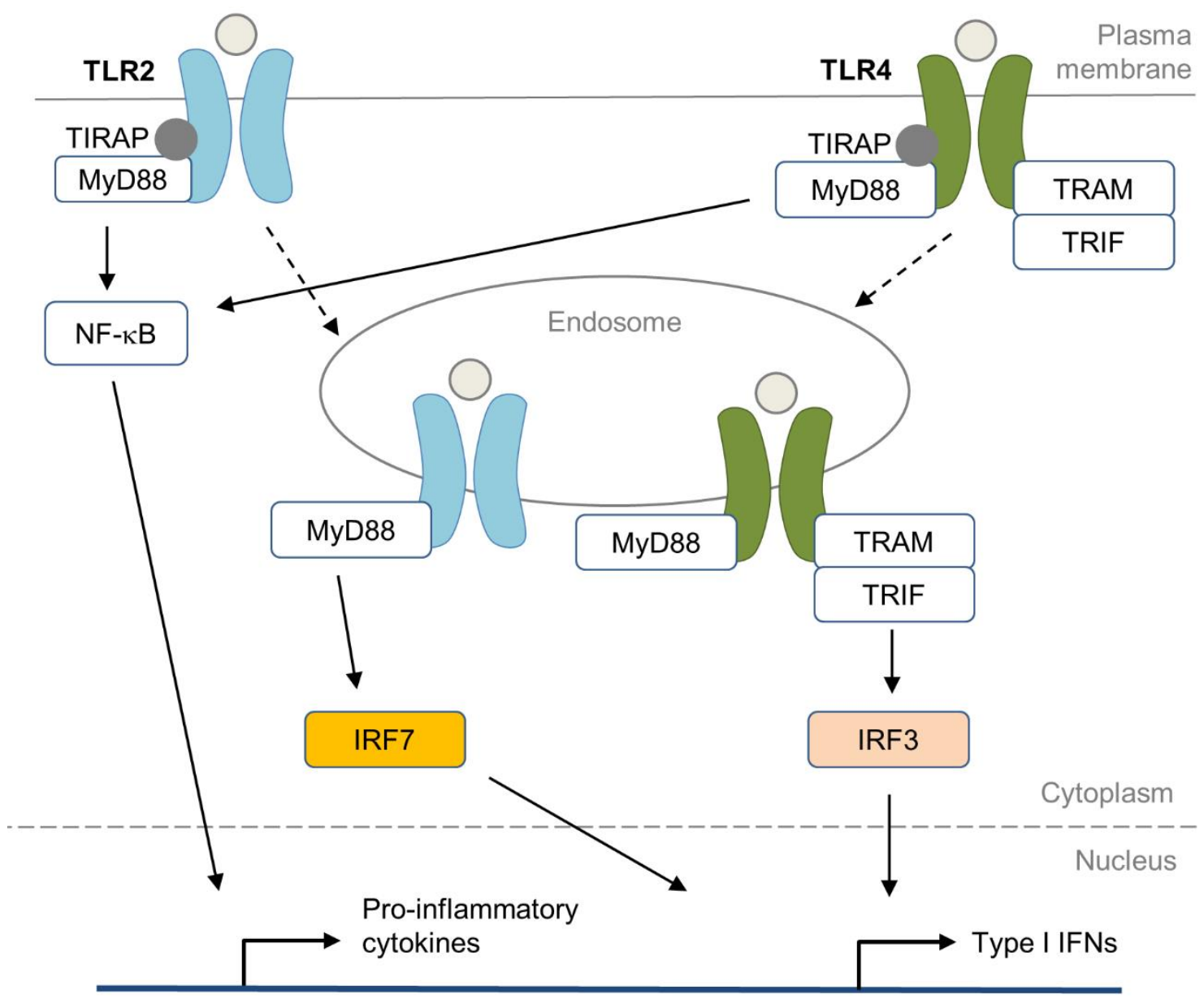

Figure 3: Extracellular and intracellular TLR2 and TLR4 signaling. Figure adapted from Du et al. (2016); O'Neill et al. (2013); and Takeda \& Yamamoto (2010). Extracellular signaling of TLRs leads to transcription of inflammatory cytokines while intracellular TLR signaling leads to transcription of type I IFNs. MyD88 and TIRAP bind to the activated receptor dimer of TLR2 and TLR4 located at the cell surface, leading to the translocation of NF-KB to the nucleus and the transcription of inflammatory cytokines. When TLR2 and TLR4 are internalized, TLR2 induces the transcription of type I IFNs though MyD88 and IRF7 signaling, while TLR4 activates IRF3 upon recruitment of TRIF via TRAM, resulting in the induction of type I IFNs.

\subsection{Interferons}

Interferons (IFNs) were first described as a substance that interferes with viral infections by providing cells that have been infected with a virus before with resistance to further infections with different viruses (Isaacs \& Lindenmann, 1957). IFNs are a whole family of distinct proteins which can be divided into three different classes: type I IFNs, type II IFNs and type III IFNs. The IFNs are assigned to one of the three classes depending on which one of three distinct IFN receptor complexes they signal through. Type I IFNs bind to the heterodimeric receptor complex interferon- $\alpha / \beta$ receptor (IFNAR), consisting of the subunits IFNAR1 and 
IFNAR2. The receptor complex for type II IFNs consists of two interferon gamma receptor (IFNGR1 and IFNGR2) heterodimers forming a dimer. Type III IFNs act through a heterodimer consisting of IL-10R2 and interferon lambda receptor (IFNLR) 1. The IL-10R2 is shared by type III IFNs with the structurally similar cytokines IL-10, IL-22 and IL-26, whereas the IFNLR1 is only used by type III IFNs.

The only member of the type II IFN family is IFN- $\mathrm{\gamma}$, a cytokine with pro-inflammatory and immunomodulatory functions distinct from the other interferon classes (Schroder et al., 2004). Type III interferons are the most recently discovered class of interferons and consist of the four subtypes IFN- $\lambda 1$ (IL-29), IFN- $\lambda 2$ (IL-28A), IFN- $\lambda 3$ (IL-28B), and IFN- $\lambda 4$ (Kotenko et al., 2003; Sheppard et al., 2003). Their antiviral, anti-proliferative, and immunomodulatory functions are similar to those of type I interferons (Lazear et al., 2019). Type I IFNs found in mammals include IFN- $\alpha$, IFN- $\beta$, IFN- $\varepsilon$, IFN- $\kappa$ and IFN- $\omega$. The best described type I IFNs are IFN- $\alpha$ and IFN- $\beta$. While only a single IFN- $\beta$ exists in mice and humans, there are 13 known subtypes of IFN- $\alpha$ in humans and 14 in mice.

Type II IFN is mostly produced by T-cells and natural killer cells and plays a role in anti-viral defence and anti-tumor immune responses. Furthermore it has been reported to act in concert with type I IFNs and amplify their anti-viral activity (A. J. Lee \& Ashkar, 2018). Meanwhile, the signaling pathways and transcriptional programs of type I and III IFNs are highly similar to each other, albeit utilizing distinct receptors (Lazear et al., 2019). They are furthermore induced by similar pathogen sensing pathways. However, the subcellular location of PAMP recognition has been shown to affect the type of IFN being produced, at least for TLR4. While endolysosomal TLR4 signaling induces production of type I IFNs, TLR4 signaling from the plasma membrane leads to the production of type III IFNs (Kagan et al., 2008; Odendall et al., 2017). Differences between type I and III IFNs arise mostly due to the distinct distribution of their respective receptors. While the IFNAR receptor for type I IFNs is expressed by most cell types, including leukocytes, fibroblasts and endothelial cells, expression of the IFNLR receptor of type III IFNs is mostly limited to epithelial cells (D. E. Levy et al., 2011; Sommereyns et al., 2008; Stanifer et al., 2019). Furthermore, the type I IFN response is more rapid and potent than the type III IFN response.

\subsubsection{Induction and downstream signaling of type I IFNs}

IFNs are not only induced in response to viral infections, but also in response to detection of microbes by PRRs such as TLRs (Boxx \& Cheng, 2016). TLR-mediated induction of type I IFNs utilizes different signaling pathways, either MyD88-dependent or TRIF-dependent and 
has been shown to be spatio-temporally regulated (Perkins \& Vogel, 2015). The members of the interferon-regulatory factor (IRF) family play a pivotal role in the regulation of the production of type I IFNs at the gene transcription level. Nine IRF proteins, namely IRF1-9, exist in mammals. They all have a conserved DNA-binding domain which recognizes the IFN-stimulated regulatory element (ISRE) found within the promoter of IFNs and IFNstimulated genes (ISGs). The first IRF to be discovered was IRF1, which is recruited by the adaptor MyD88, but has been shown to be non-essential for the induction of IFNs in some settings, while being essential in others (Colonna, 2007; Matsuyama et al., 1993; Miyamoto et al., 1988). IRF3 and IRF7 share great structural homologies and are generally considered the master regulators of the transcription of type I IFNS, while IRF9 regulates the transcription of ISGs downstream of type I IFNs (Honda, Yanai, Negishi, et al., 2005; Ning et al., 2011; Paul et al., 2018). IRF3 is expressed ubiquitously and binds to ISRE in the promoter of IFN- $\alpha$ and IFN- $\beta$ upon activation by phosphorylation (Sharma et al., 2003; Yanai et al., 2018). IRF3 is activated at intracellular vesicles by adaptor proteins such as TRIF in the case of TLR3 and TLR4. Unlike IRF3, basal levels of IRF7 are very low (Sato et al., 2000). However, IRF7 is produced upon induction by type I IFNs, resulting in a feedforward response further upregulation the expression of type I IFNs (Marie, 1998). Upon activation by phosphorylation, IRF7 can induce transcription of IFN- $\alpha$ and IFN- $\beta$ by forming either homodimers or heterodimers with IRF3 (Honda, Yanai, Negishi, et al., 2005).

Upon production, type I IFNs bind to their receptor, thereby activating the JAK-STAT signaling pathway, resulting in the transcription of ISGs (Ivashkiv \& Donlin, 2014). JAK proteins are bound to the cytoplasmic domains of the IFN receptor complexes in an inactive conformation. Ligand binding to the IFN receptor complex leads to the activation of the JAK proteins, which then phosphorylate the IFN receptor chain, enabling STATs to bind via their SRC homology 2 (SH2) domains (Heim et al., 1995). This leads to the phosphorylation of the STAT proteins, resulting in their release from the receptor. The STAT proteins involved in IFN signaling are STAT1 and STAT2. The activated STATs form STAT1 homodimers in case of type II IFN signaling or heterodimers consisting of STAT1 and STAT2 in case of type I and III signaling (Figure 4). The STAT1 homodimers translocate to the nucleus and bind to gamma-activated sequences (GAS), leading to the transcription of downstream ISGs. The STAT1/STAT2 heterodimers bind to IRF9, forming a complex called ISG factor 3 (ISGF3). The ISGF3 complex then translocates to the nucleus and binds to ISREs, inducing the transcription of downstream ISGs with diverse functions, which are reviewed in detail in "Interferon-Stimulated Genes: A complex Web of Host Defenses" (Schneider et al., 2014) and shall be described here briefly. 
Type I IFN

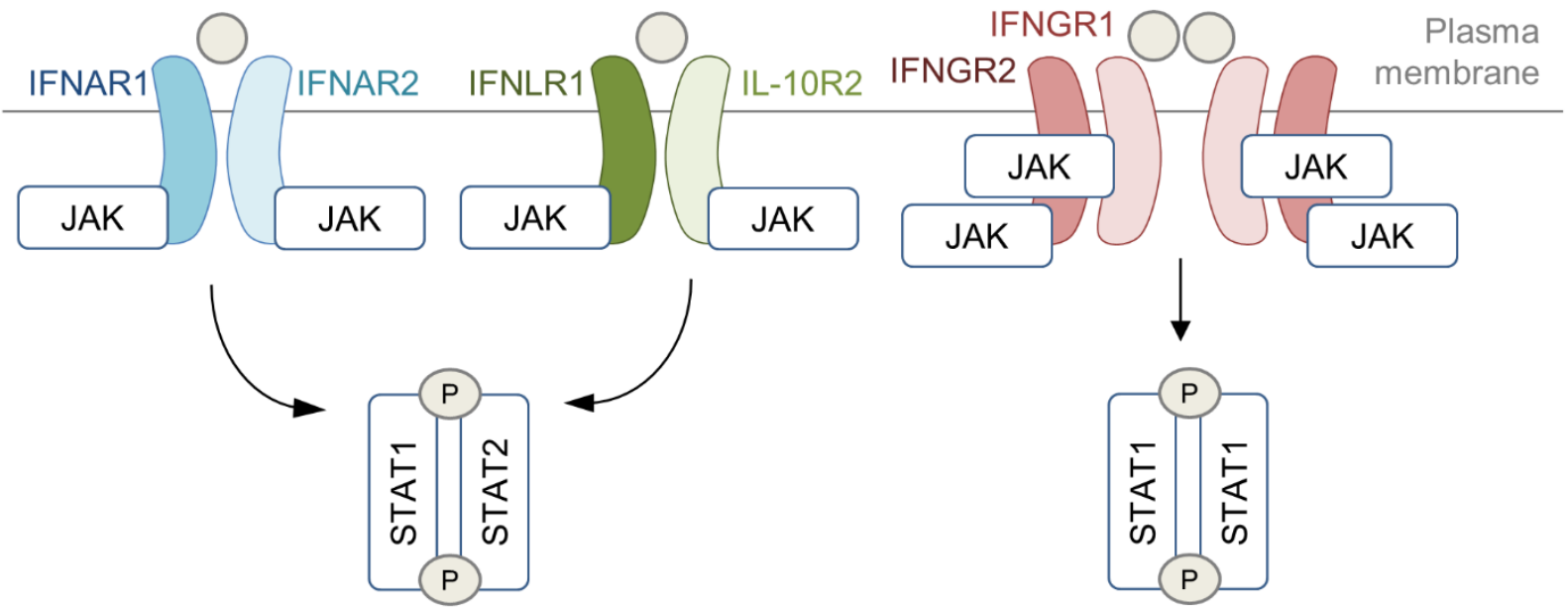

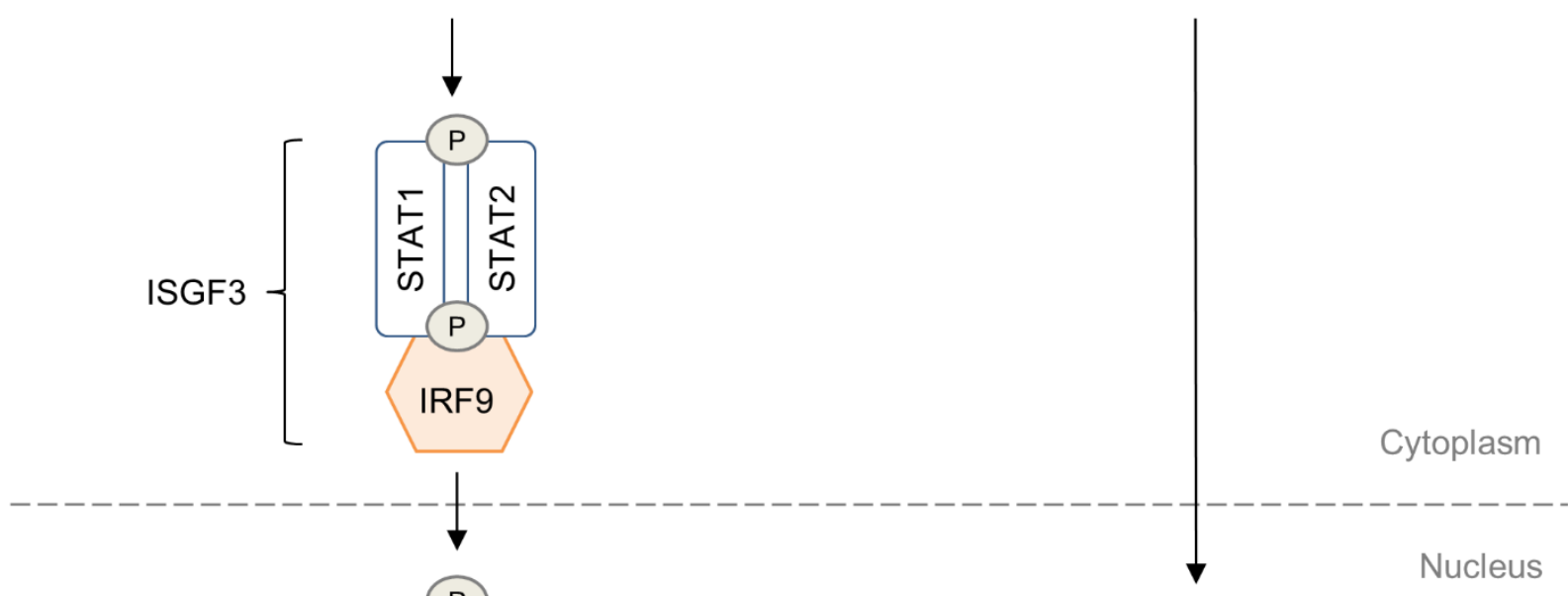

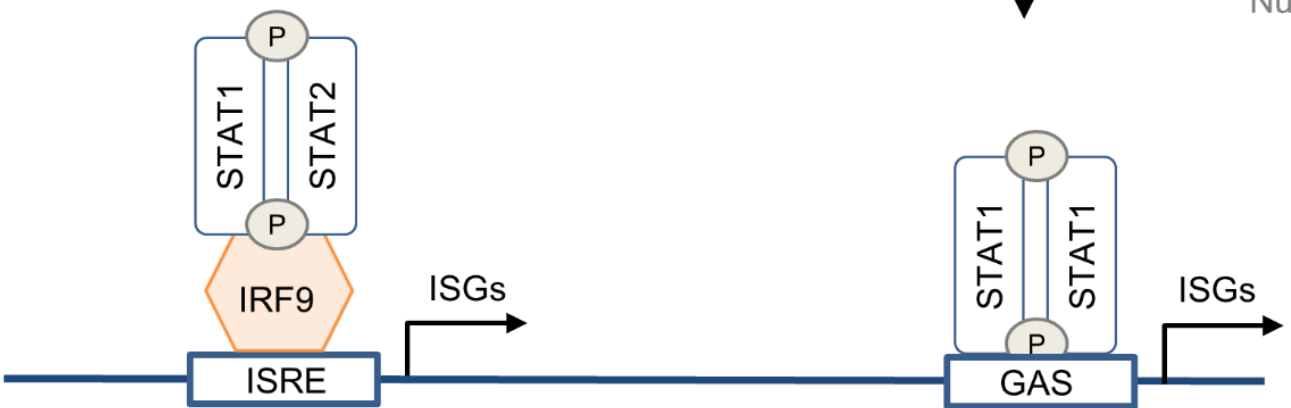

Figure 4: Downstream signaling cascade of IFNs. Figure adapted from Schneider et al. (2014). The three classes of IFNs utilize three distinct receptor complexes: type I IFNs signal through IFNAR1 and IFNAR2 heterodimers, type III IFNS signal through IFNLR1 and IL-10R heterodimers, and type II IFNs signal through dimers of IFNGR1 and IFNGR2 heterodimers. Binding of type I and type III IFNs triggers phosphorylation of JAKs bound to the receptor complexes, which leads to the phosphorylation of recruited STAT1 and STAT2. STAT1 and STAT2 then form a heterodimer and bind to IRF9. The complex of STAT1, STAT2 and IRF9 is referred to as ISGF3. ISGF3 translocates to the nucleus and induces gene expression regulated by ISRE promoter elements. Binding of type II IFN dimers to their receptor complex also leads to phosphorylation of associated JAKs, and then phosphorylation of STAT1, but not STAT2. STAT1 homodimers then translocate to the nucleus and induce gene expression regulated by GAS promoter elements. 
The best described role of IFNS is the interference with viruses, which is achieved by a wide range of ISGs. Indeed, for every stage of the life cycle of viruses, from the entry into the host cell, to translation and replication of the viral genome, and finally the assembly and the egress of the virus, ISGs are known that inhibit the viral activity. Examples for these anti-viral effectors are Myxovirus resistance (Mx) proteins, interferon-induced transmembrane (IFITM) proteins, tripartite motif family (TRIM) proteins and oligoadenylate synthase (OAS) proteins. The type I IFN response has also been studied in infections with intracellular and extracellular bacteria with ambiguous outcome; in some studies, type I IFNs have been shown detrimental, in others beneficial to host defence (McNab et al., 2015). These effects are due to the induction of ISGs with the ability to modulate innate and adaptive immune responses. Reported effects of type I IFNs in bacterial infections include regulation of apoptosis and necroptosis, promotion as well as suppression of immune cell recruitment, influence on bacterial clearance, suppression of pro-inflammatory response and promotion of anti-inflammatory response, including promotion of IL-10 response, and furthermore promotion of tissue integrity. These effects are reviewed in detail in "The Roles of Type I Interferon in Bacterial Infection" (Boxx \& Cheng, 2016).

While PRRs, JAKs, STATs and IRFs are present at baseline, facilitating a rapid IFN response, they are also ISGs themselves and their expression is enhanced in response to IFN, reinforcing IFN signaling in a positive feedback loop and sensitizing cells for enhanced recognition of pathogens. Production of cytokines induced by type I IFNs furthermore enables the communication with neighbouring cells. Finally, some of the ISGs desensitize the cells to IFN signaling to enable the return to homeostasis. Next to desensitization by cellintrinsic mechanisms, such as receptor internalisation and turnover rate, some ISGs are able to inhibit the JAK-STAT signaling pathway, such as ubiquitin specific peptidase (USP) 18, which interacts with IFNAR2 and thereby prevents binding of JAK1 to the receptor complex (Coccia et al., 2006; Malakhova et al., 2006).

Albeit their role as a central pillar in host defence, type I IFNs have been shown to be absent in mast cells after non-viral induction (Dietrich, Rohde, et al., 2010; Keck et al., 2011). 


\subsection{Aim of thesis}

While pro-inflammatory functions of mast cells are well known, their role during the resolution of inflammation is still unclear. To elucidate the role of mast cells during the resolution of a local inflammation, the first aim of this thesis was to identify the effect of mast cells on their neighbouring cells in the tissue (Figure 5). In a first step, a suitable model of a local inflammation had to be chosen. Comparison of the two most commonly used TLR-agonists for locally-induced inflammations LPS and zymosan revealed a more systemic response to LPS, which left zymosan as the suitable candidate. To identify neighbouring cells of mast cells and therefore cells potentially influencing and being influenced by them, multi epitope ligand cartography (MELC) was performed next in naïve paws, as well as during peak and resolution of inflammation. Since macrophages were identified as the prominent neighbours of mast cells during resolution of inflammation, differences regarding macrophage populations in mast cell-deficient mice and control mice were screened by MELC analysis. This indicated for a decreased efferocytosis of neutrophils by macrophages in the absence of mast cells. To validate this effect, paw tissue was analysed by multiparametric flow cytometry regarding differences in phagocytosis and efferocytosis.

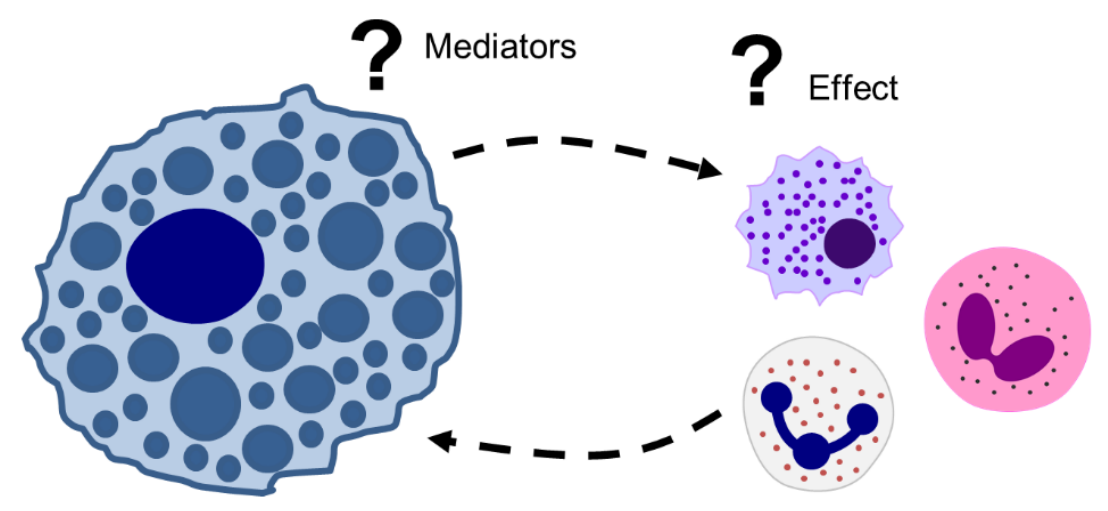

Figure 5: Aims of this thesis. The aims of this thesis are 1) to study the effect of mast cells on their neighbouring cells and 2) to identify mediators released by mast cells during the resolution of inflammation. Neighbouring cells have the potential to influence mast cells and vice versa by releasing mediators, which may alter their phenotype and affect the progression of the inflammation.

The second aim of this thesis was to identify mediators produced by mast cells during the resolution of inflammation (Figure 5). Therefore, levels of cytokines in the paws of zymosaninduced mast cell-deficient mice and control mice were analysed, and an untargeted screening of mediators produced in response to zymosan was performed by mRNA sequencing of bone marrow-derived mast cells. Finally, the results of the sequencing were validated on a protein level. Combined, these results were intended to contribute to a better understanding of the phenotype of mast cells and their effect on the resolution of inflammation. 


\title{
2. Materials and Methods
}

\author{
2.1. Materials
}

\subsubsection{Animals}

C57BL/6N mice were supplied by Janvier (Le Genest, France). Mcpt5-DTA-Cre mice were originally described by Axel Roers' group at Technische Universität Dresden (Dudeck et al., 2011). Sex and age matched Mcpt5-DTA-Cre- litter mates were used as control mice for Mcpt5-DTA-Cre ${ }^{+}$mice. The animals were cared for corresponding to International Association for the Study of Pain guidelines (Grant FK1065, FK1093 and FK1138). For all experiments, the ethics guidelines for investigations in conscious animals were obeyed and the procedures were approved by the local ethics committee (Regierungspräsidium Darmstadt). The animals had free access to food (Sniff standard diet) and water and were maintained in climate- $\left(23 \pm 0.5^{\circ} \mathrm{C}\right)$ and light-controlled rooms (light from 7.00 a.m. to 7.00 p.m.).

2.1.2. Antibodies for flow cytometry (FC) and MELC

\begin{tabular}{lccl}
\hline Name & \multicolumn{2}{c}{ Dilution } & Supplier, Product \# \\
& \multicolumn{1}{c}{ FC } & MELC & \\
\hline CD3-APC & $1: 50$ & & Miltenyi, \#130-122-943 \\
\hline CD11b-APC/Cyanine7 & $1: 50$ & & Biolegend, \#101225 \\
\hline CD11b-FITC & & $1: 400$ & Bio-Rad, \#MCA74F \\
\hline CD11c-PE & & $1: 100$ & Miltenyi, \#130-102-799 \\
\hline CD11c-PerCP & $1: 50$ & & Biolegend, \#117326 \\
\hline CD11c-FITC & $1: 50$ & & eBioscience, \#11-0114-85 \\
\hline CD11c-VioBlue & $1: 50$ & & Miltenyi, \#130-102-797 \\
\hline CD31-PE & & $1: 400$ & BD Biosciences, \#553373 \\
\hline CD45-FITC & & $1: 200$ & Miltenyi, \#130-091-609 \\
\hline CD45-VioGreen & $1: 25$ & & Miltenyi, \#130-123-900 \\
\hline CD80 & & $1: 100$ & Biolegend, \#104706 \\
\hline CD86-PE & & $1: 200$ & Biolegend, \#105008 \\
\hline
\end{tabular}




\begin{tabular}{|c|c|c|c|}
\hline CD86-BV421 & $1: 50$ & & Biolegend, \#105031 \\
\hline CD117-PE & $1: 50$ & & Miltenyi, \#130-102-795 \\
\hline CD117-Cy5 & & $1: 50$ & Bioss, \#bs-10005R-Cy5 \\
\hline CD206-APC & $1: 50$ & $1: 200$ & Biolegend, \#141708 \\
\hline $\mathrm{F} 4 / 80$ & & $1: 400$ & Biolegend, \#123107 \\
\hline F4/80-PE & $1: 50$ & & Biolegend, \#123110 \\
\hline F4/80-PE/Cyanine7 & $1: 50$ & & Biolegend, \#123113 \\
\hline FceRla-FITC & $1: 50$ & & Biolegend, \#134305 \\
\hline IFN- $\beta$ & & $1: 100$ & Novus Biologicals, \#nbp1-77288 \\
\hline IL-1 $\beta-F I T C$ & & $1: 100$ & Thermo Fisher, \#11-7114-82 \\
\hline IL-4-AlexaFluor488 & & $1: 50$ & Biolegend, \#504109 \\
\hline IL-10-FITC & & $1: 100$ & eBioscience, \#11-7101 \\
\hline IL-13-AlexaFluor488 & & $1: 50$ & Invitrogen, \#53-7133-82 \\
\hline Ly6C-APC & & $1: 400$ & eBioscience, \#17-5932-80 \\
\hline Ly6C-eFluor450 & $1: 50$ & & eBioscience, \#48-5932-82 \\
\hline Ly6C-PE & $1: 50$ & & eBioscience, \#12-5932-82 \\
\hline Ly6G/Ly6C-FITC (Gr1) & $1: 50$ & $1: 400$ & eBioscience, \#11-5931-82 \\
\hline MHCII-APCeFluor770 & $1: 25$ & & eBioscience, \#47-5321-80 \\
\hline MHCII-PE & & $1: 50$ & Miltenyi, \#120-000-810 \\
\hline Propidium iodide (PI) & & $1: 800$ & Sigma-Aldrich, \#P4170 \\
\hline Siglec F-PE & $1: 50$ & $1: 200$ & BD Pharmingen, \#562068 \\
\hline Siglec F-PEvio770 & $1: 25$ & & Miltenyi, \#130-102-167 \\
\hline
\end{tabular}

2.1.3. Proteins, Enzymes and Antibodies

\section{Name}

Collagenase from Clostridium histolyticum

DNP-BSA (Dinitrophenyl-BSA protein Conjugate)

DNP-specific IgE (Monoclonal Anti-Dinitrophenyl

antibody produced in mouse)

IFNAR1 monoclonal antibody (MAR1-5A3, isotype IgG1 eBioscience, \#16-5945-85 kappa), functional grade

\section{Supplier, Product \#}

Sigma-Aldrich, \#C9891-1G

Alpha diagnostic, \#DNP35-N-10

Sigma-Aldrich, \# D8406

Bio X Cell, \#BE0241 
InVivoMAb anti-mouse IgG1 isotype control

Isotype control lgG1 kappa, Cytochrome C (6H2)
Bio X Cell, \#BE0083

Santa Cruz Biotechnology

\#sc-13561

PeproTech, \#AF-315-02

PBL Assay Science, \#12405-1

PeproTech, \# 213-13-100UG

Peprotech, \#213-13

\subsubsection{Solutions and chemicals}

\begin{tabular}{ll}
\hline Name & Supplier, Product \# \\
\hline 1-palmitoyl-2-hydroxy-sn-glycero-3-phosphocholine & Avanti Polar Lipids, \#855675-25 \\
(16:0 LysoPC) & \\
\hline 2-mercaptoethanol & AppliChem, \#A1108 \\
\hline Bafilomycin A1 Ready Made solution in DMSO & Sigma-Aldrich, \#SML1661 \\
\hline Bicinchoninic acid (BCA) & Sigma-Aldrich, \#B9643 \\
\hline Brefeldin A from Penicillium brefeldianum & Sigma-Aldrich, \#B6542-5MG \\
\hline cOmplete TM Protease Inhibitor Cocktail & Roche, \#11697498001 \\
\hline Cytochalasin D from Zygosporium mansonii & Sigma-Aldrich, \#C2618 \\
\hline Fetal Bovine Serum (FBS) & Gibco, \#41F9730K \\
\hline GSK717 (NOD2 Signaling Inhibitor II) & Calbiochem, \#533718 \\
\hline L-Glutamine 200 mM & Sigma-Aldrich, \#G7513 \\
\hline Lipopolysaccharides (LPS) Escherichia coli O127:B8 & Sigma-Aldrich, \#L3129 \\
\hline MEM non essential amino acids & Gibco, \#11140-035 \\
\hline NaOH (Sodium hydroxide pellets) & AppliChem, \#A1551 \\
\hline Penicillin/Streptomycin & Invitrogen, \#P35364 \\
\hline
\end{tabular}




\begin{tabular}{ll}
\hline RPMI 1640 & Gibco, \#31870 \\
\hline RPMI 1640 GlutaMAX & Gibco, \#61870036 \\
\hline Saponin & Roth, \#4185.1 \\
\hline Sodium Pyruvate & Gibco, \#11360-039 \\
\hline Sphingosine-1-phosphate (S1P) & Cayman Chemicals, \#62570 \\
\hline Tissue Extraction Reagent I & Invitrogen, \#FNN0071 \\
\hline Tissue Tek O.C.T Compound Containing & Sakure, \#4583 \\
\hline TRIS-hydrochlorid (TRIS-HCl) & Roth, \#9090.3 \\
\hline U0126 (MEK1 and MEK2 inhibitor) & Cell Signaling Technology, \#9903 \\
\hline WEB 2086 (PAF receptor antagonist) & Tocris, \#2339 \\
\hline Zymosan A from Saccharomyces cerevisae & Sigma-Aldrich, \#Z4250-1G
\end{tabular}

\subsubsection{Media and buffers}

$\mathrm{H}_{2} \mathrm{O}$ was used as solvent, if not explicitly stated otherwise.

\begin{tabular}{lll} 
Name & Composition & Concentration \\
\hline ACK & $\mathrm{NH}_{4} \mathrm{CL}$ & $135 \mathrm{mM}$ \\
(erythrocyte lysis buffer) & $\mathrm{NaHCO}_{3}$ & $10 \mathrm{mM}$ \\
& $\mathrm{Na-EDTA}$ & $0,1 \mathrm{mM}$ \\
& $\mathrm{pH} \mathrm{7,2}$ & \\
\hline FACS Collagenase buffer & Collagenase & $3 \mathrm{mg} / \mathrm{ml}$ \\
& in DMEM & \\
\hline FACS staining buffer & FBS & $1 \%(\mathrm{v} / \mathrm{v})$ \\
& in PBS & $10 \%(\mathrm{v} / \mathrm{v})$ \\
\hline FACS stop solution & FBS & \\
& in DMEM & $10 \%(\mathrm{v} / \mathrm{v})$ \\
& & $100 \mathrm{U} / \mathrm{ml}$
\end{tabular}


M-CSF

$20 \mathrm{ng} / \mathrm{ml}$

in RPMI1640 GlutaMAX

\begin{tabular}{lll}
\hline Mast cell medium & FBS (heat inactivated) & $10 \%(\mathrm{v} / \mathrm{v})$ \\
& L-Glutamine $200 \mathrm{mM}$ & $4 \mathrm{mM}$ \\
& Penicillin/Streptomycin & $100 \mathrm{U} / \mathrm{ml}$ \\
& Sodium Pyuvate & $1 \mathrm{mM}$ \\
& MEM non essential amino acids & $1 \%(\mathrm{v} / \mathrm{v})$ \\
2-mercaptoethanol & $50 \mu \mathrm{M}$ \\
Recombinant murine IL-3 & $10 \mu \mathrm{g} /$ liter \\
in RPMI1640, filter sterilize & \\
\hline Tyrode's buffer & HEPES & $10 \mathrm{mM}$ \\
NaCl & $129 \mathrm{mM}$ \\
KCL & $3 \mathrm{mM}$ \\
CaCl2 & $1,4 \mathrm{mM}$ \\
MgCl2 & $1 \mathrm{mM}$ \\
Glucose & $5,6 \mathrm{mM}$ \\
BSA & $0,1 \%(\mathrm{w} / \mathrm{v})$
\end{tabular}

2.1.6. Sample Processing Kits

Name

\begin{tabular}{ll}
\hline Bioanalyzer High Sensitivity DNA Assay & Agilent, \#5067-4626 \\
\hline Bio-Plex Pro mouse cytokine group I & Bio-Rad, \# \#M60009RDPD \\
\hline IL-4 (mouse) ELISA Kit & Enzo, \#ADI-900-043 \\
\hline KC/CXCL1 Mouse ELISA Kit & Invitrogen, \#EMCXCL1 \\
\hline Mouse IFN- $\alpha$ All Subtype ELISA Kit & PBL Assay Science, \#42115-1 \\
\hline Mouse IFN- $\beta$ Quantikine ELISA Kit & R\&D Systems, \#MIFNB0 \\
\hline Mouse IL-10 Quantikine ELISA Kit & R\&D Systems, \#M1000B \\
\hline Mouse IL-13 ELISA Kit & BioOcean, \#EK213 \\
\hline Mouse IL-1 $\beta$ Quantikine ELISA Kit & R\&D Systems, \#MLB00C \\
\hline
\end{tabular}




\begin{tabular}{ll}
\hline QuantSeq 3' mRNA-Seq Library Prep Kit FWD for & Lexogen, \#015 \\
Illumina & \\
\hline Qubit $^{\text {TM }}$ dsDNA HS Assay Kit & Invitrogen, \# Q32851 \\
\hline RNeasy Micro Kit & Qiagen, \#74004
\end{tabular}

\subsubsection{Laboratory equipment}

\begin{tabular}{ll} 
Name & Supplier \\
\hline 2100 Bioanalyzer Instrument & Agilent Technologies \\
\hline Bio-Plex 200 & Bio-Rad \\
\hline $\mathrm{CO}_{2}$ Incubator BBD 6220 & Heraeus, Thermo Fisher Scientific \\
\hline Cryostat CM3050 S & Leica \\
\hline Cryotom CM3050S & Leica \\
\hline DynaMag-96 (\#12331D) & Invitrogen \\
\hline Dynamic Plantar Aesthesiometer & Ugo Basile \\
\hline FACS Aria cell sorter & BD Biosciences \\
\hline FACS Canto II & BD Biosciences \\
\hline IKA MTS 4 MTP Microplate Shaker & IKA \\
\hline NextSeq500 & Illumina \\
\hline Plethysmometer & ITC Life Science, USA \\
\hline Qubit Fluorometer & Thermo Fisher Scientific \\
\hline Tecan Infinite F200 Pro Microplate Reader & Bandelin \\
\hline Ultrasonic Homogenizer SONOPULS HD2070 MS73 Sciences \\
\hline
\end{tabular}




\subsubsection{Software}

Name

Cell Profiler version 3.1.9

FlowJo V10

GraphPad Prism 6

HistoCAT version 1.76

ImageJ 1.52v

Zeiss Zen
Supplier/Developer

Broad Institute

BD Biosciences

GraphPad Software

BodenmillerGroup

Wayne Rasband

Carl Zeiss 


\subsubsection{Mechanical nociception and paw edema measurement in mice}

Mechanical nociception and paw edema were analysed as described in Hahnefeld \& Kornstädt et al. (2021) and Kornstädt et al. (2021). To induce an inflammation, $10 \mu \mathrm{l}$ zymosan (3 mg/ml or $12 \mathrm{mg} / \mathrm{ml}$ in PBS, \#Z4250, Sigma-Aldrich) or LPS (100 $\mu \mathrm{g} / \mathrm{ml}$ in PBS, \# L3129, Sigma-Aldrich) was injected subcutaneously into the plantar side of one hind paw. The contralateral hind paw served as negative control. Before behavioural experiments, the mice were habituated for two consecutive days to the test room and the test setting. For assessment of mechanical nociception, the latency of paw withdrawal was measured using a plantar aesthesiometer (Dynamic Plantar Aesthesiometer, Ugo Basile). A force range of 0 to $5 \mathrm{~g}$ with a ramp of $0,5 \mathrm{~g} / \mathrm{s}$ was applied with a steel rod of $2 \mathrm{~mm}$ in diameter, until a withdrawal reaction appeared. The volume of the edema was measured by immersion of the mouse paw using a phlethysmometer (IITC Life Science). Behavioural tests were performed at baseline and at the indicated time points after induction of inflammation.

\subsubsection{Cytological and histological analysis}

\subsubsection{Multi Epitope Ligand Cartography (MELC)}

MELC technology is an automated immunohistological imaging method and can be used to visualize a high number of antibodies on the same sample as described before (Kern et al., 2018; Kornstädt et al., 2021; Pierre et al., 2017; Pierre \& Scholich, 2010). Briefly, tissues were embedded in tissue freezing medium (Tissue-Tek O.C.T. Compound, \#4583, Sakura Finetek B.V.), cryosections of $10 \mu \mathrm{m}$ thickness were applied on silane-coated coverslips, fixed in 4\% paraformaldehyde in PBS for 15 min, permeabilized with $0.1 \%$ Triton X100 in PBS for 15 min and blocked with 3\% BSA in PBS for 1 hour. The sample was placed on the stage of a Leica DM IRE2 and a picture was taken. Then, in an automated procedure, the sample was incubated for $15 \mathrm{~min}$ with bleachable fluorescence-labelled antibodies and rinsed with PBS. Afterward, the phase contrast and fluorescence signals were imaged by a cooled charge-coupled device camera (Apogee KX4, Apogee Instruments). A bleaching step was performed to delete fluorescence signals, and the post-bleaching image was recorded. Then the next antibody was applied. For data analysis, fluorescence images produced by each antibody were aligned pixel-wise and were corrected for illumination faults using flat- 
field correction. The post-bleaching images were subtracted from their following fluorescence image. Antibodies and concentrations used for MELC analysis are listed in 2.1.2. Antibodies for flow cytometry (FC) and MELC.

\subsubsection{Analysis of MELC data}

MELC data was analysed as described in Kornstädt et al. (2021). In a first step all greyscale antibody channel images were processed using ImageJ $1.52 \mathrm{v}$ to diminish noise, background fluorescence and remove artefacts for further analyses if necessary. Subsequently, images for propidium iodide (cell nuclei) and CD45 were used for single cell segmentation using Cell Profiler (version 3.1.9) (McQuin et al., 2018). The resulting segmentation mask were loaded into histoCAT (version 1.76) (Schapiro et al., 2017) together with the corresponding antibody channel images.

All images, excluding zymosan images and images used for single cell mask generation, were z-score normalized and used for Barnes-Hut t-SNE (BH t-SNE) (Amir et al., 2013) and PhenoGraph analysis (Levine et al., 2015) as implemented in histoCAT. PhenoGraph defines cell cluster based on single cell mask and marker colocalization ( $\mathrm{k}$ was set to 20 or 30 ). $\mathrm{BH} \mathrm{t}-$ SNE scatter plot was overlaid with colored PhenoGraph cluster map. To investigate the relationship between clusters, neighbourhood analysis under standard conditions as implemented in histoCAT was used (Schapiro et al., 2017).

MELC and analysis of MELC data was performed by Tim Schäufele and Anja Kolbinger.

\subsubsection{Polychromatic flow cytometry of immune cells in paw of mice}

For analysis of immune cells in inflamed paws polychromatic flow cytometry was performed as described in Hahnefeld \& Kornstädt et al. (2021) and Kornstädt et al. (2021). In general, zymosan- or LPS-induced edema were dissected after killing of the mice and transferred to $500 \mu \mathrm{PBS}$ on ice. The edema were cut into $<1 \mathrm{~mm} 3$ pieces, transferred into $500 \mu$ lysis buffer $\left(3 \mathrm{mg} / \mathrm{ml}\right.$ Collagenase in DMEM) and incubated for $45 \mathrm{~min}$ at $37^{\circ} \mathrm{C}$. Tissue lysis was stopped by adding $5 \mathrm{ml}$ lysis stop buffer (10\% FBS in DMEM) and the suspension was passed through a $70 \mu \mathrm{m}$ cell strainer to create single cell suspensions. The cells were then centrifuged at $1000 \mathrm{rcf}$ for $5 \mathrm{~min}$; afterwards the supernatant was discarded and the cells were resuspended and incubated in erythrocyte lysis (ACK) buffer for $5 \mathrm{~min}$ at room temperature. Residual cells were collected by centrifugation (1000 rcf, $5 \mathrm{~min}$ ) and 
resuspended in $120 \mu \mathrm{l}$ PBS. Cells were then transferred into a 96 well V-bottom plate and centrifuged at $1000 \mathrm{rcf}$ for $5 \mathrm{~min}$. The supernatant was discarded and the cells were resuspended in $30 \mu \mathrm{l}$ FACS buffer (1\% FBS in PBS). Then, $20 \mu \mathrm{l}$ of antibody mastermix were added and the cells were incubated for 1 hour at $4^{\circ} \mathrm{C}$ for staining of surface molecules. After the staining, the cells were washed with FACS buffer and resuspended in PBS and transferred to FACS tubes for subsequent FACS analysis. Samples were kept in the dark and on ice until analysis. They were acquired with a FACS Canto II flow cytometer and analysed using FlowJo software V10.4 (both BD Life Sciences). For gating, unstained controls and fluorescence minus one (FMO) controls were used. Concentrations of the single antibodies are listed in 2.1.2. Antibodies for flow cytometry (FC) and MELC.

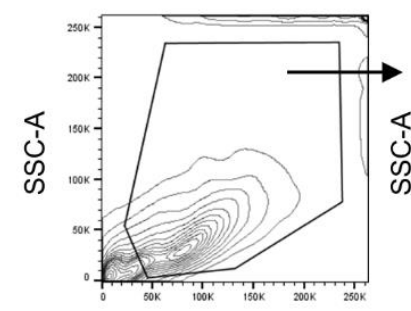

FSC-A

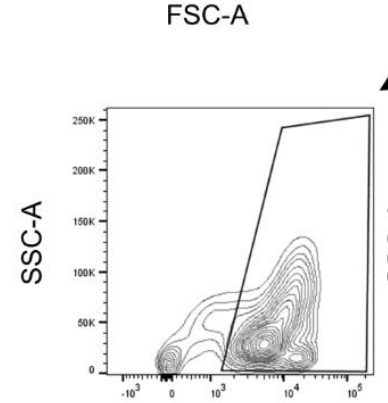

Ly6C

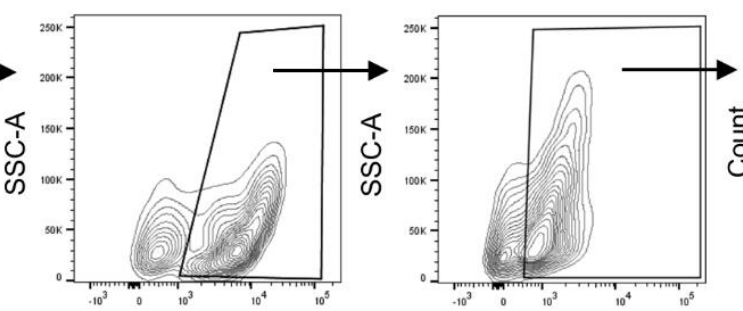

$\mathrm{F} 4 / 80$

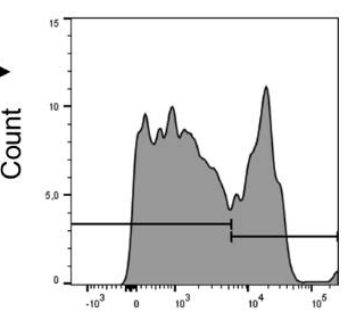

Ly6C

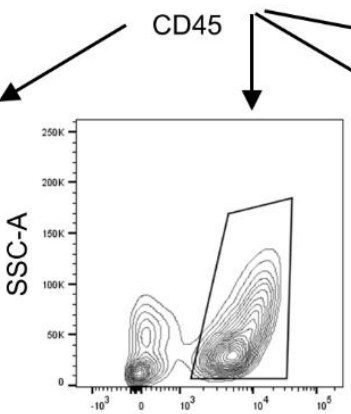

Ly6G-GR1

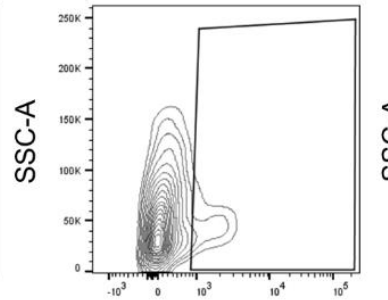

CD11c

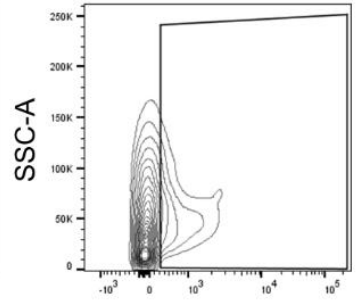

Siglec F

Figure 6: Gating strategy for identification of immune cells in the inflamed paw of mice $5 \mathrm{~h}$ after intraplantar injection of zymosan or LPS. Adapted from Hahnefeld \& Kornstädt et al. (2021). Cells were gated based on their side scatter area (SSC-A) and forward scatter area (FSC-A). Within the CD45+ immune cells,

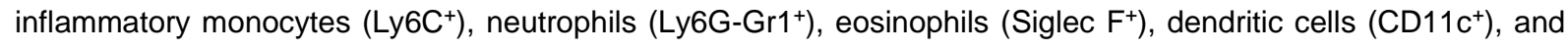
macrophages $\left(\mathrm{F} 4 / 80^{+}\right)$were identified. Within the $\mathrm{F} 4 / 80^{+}$gate, inflammatory macrophages were identified as Ly6C+ cells.

For comparison of immune cells involved in LPS- and zymosan-induced inflammation, polychromatic flow cytometry analysis was performed 5 hours after intraplantar injection of $10 \mu \mathrm{l}$ zymosan $(3 \mathrm{mg} / \mathrm{ml})$ or LPS $(100 \mu \mathrm{g} / \mathrm{ml})$. Polychromatic flow cytometry analysis was performed as describes above. Cells were stained with Ly6C-eFluor450 (eBioscience, \#485932-82), CD45-VioGreen (Miltenyi Biotec, \#130-123-900), Ly-6G/Ly-6C-FITC (eBioscience, \#11-5931-82), F4/80-PE (Biolegend, \#123110), CD11c-PerCP (Biolegend, \#117326), SiglecF-PE-Vio770 (Miltenyi Biotec, \#130-102-167), CD3-APC (Miltenyi Biotec, \#130-122-943), MHC II-APC-eFluor780 (eBioscience, \# 47-5321-82). Immune cells were identified as CD45+ 
cells, inflammatory monocytes as CD45 ${ }^{+} / \mathrm{Ly}_{6 \mathrm{C}}+$, Neutrophils as CD $45^{+} / \mathrm{Ly} 6 \mathrm{G}-\mathrm{Gr} 1^{+}$, inflammatory macrophages as $\mathrm{CD}^{2} 5^{+} / \mathrm{F} 4 / 80^{+} / \mathrm{Ly}_{6 C^{+}}$, eosinophils as $\mathrm{CD} 45^{+} /$Siglec $\mathrm{F}^{+}$, and dendritic cells as $\mathrm{CD} 45^{+} / \mathrm{CD} 11 \mathrm{c}^{+}$. The gating strategy and exemplary flow plots are shown in figure 6 .

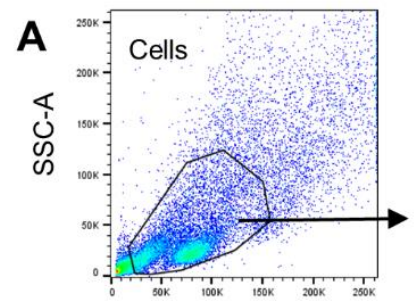

FSC-A

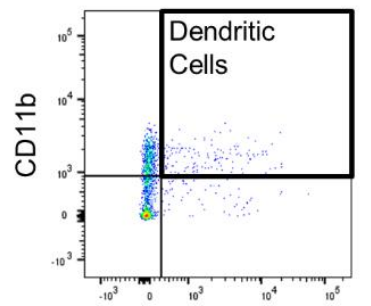

$\mathrm{MHCII}$
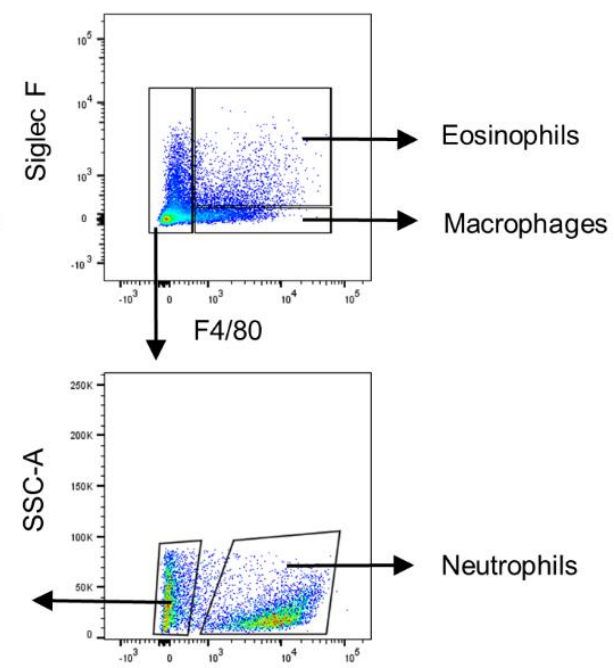

Ly6G

FMO controls:
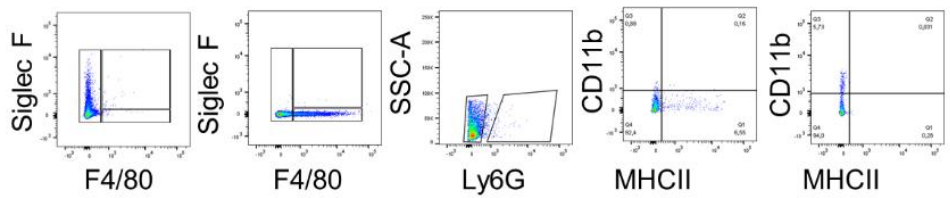

B

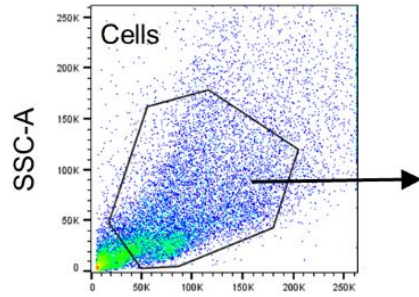

FSC-A

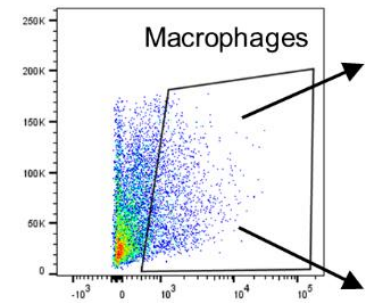

F4/80

FMO controls
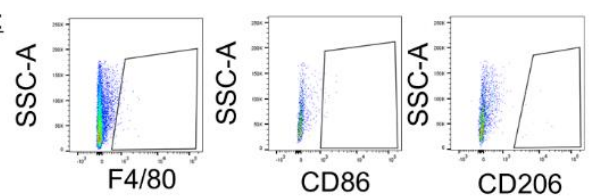

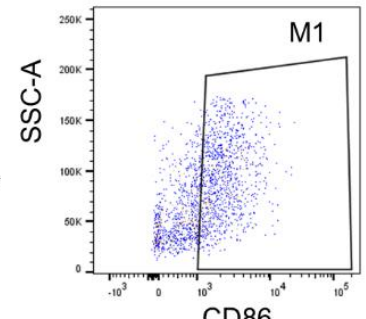

CD86

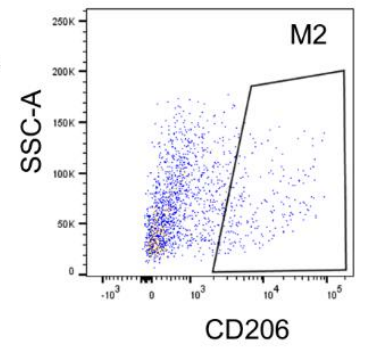

Figure 7: Gating strategy for immune cells and their subtypes in paws of Mcpt5-DTA Cre- and Cre ${ }^{+}$mice $48 \mathrm{~h}$ after injection of zymosan $(10 \mu \mathrm{l}, 12 \mathrm{mg} / \mathrm{ml})$. Reprinted from Kornstädt et al. (2021). (A) Cells were gated based on their side scatter area (SSC-A) and forward scatter area (FSC-A). Within, F4/80+ and F4/80- populations were identified. Within the $\mathrm{F} 4 / 80^{+}$cells, eosinophils were identified as Siglec $\mathrm{F}^{+}$and macrophages were identified as Siglec $\mathrm{F}^{-}$. Within the F4/80 cells, neutrophils were identified as $\mathrm{Ly}^{-} \mathrm{G}^{+}$, while Ly6G- cells were further analysed for $\mathrm{CD} 11 \mathrm{~b}^{+} \mathrm{MHCI}^{+}$dendritic cells. FMO controls are shown below the gating strategy. (B) M1 macrophages were identified as $\mathrm{CD}^{+} 6^{+}$cells among F4/80+ macrophages and M2 macrophages were identified as CD206 ${ }^{+}$cells among $\mathrm{F} 4 / 80^{+}$macrophages. $\mathrm{FMO}$ controls are shown below the gating strategy. 
For comparison of immune cell levels in Mcpt5-DTA Cre ${ }^{-}$and $\mathrm{Cre}^{+}$mice, $10 \mu \mathrm{l}$ zymosan (12 mg/ml in PBS, \#Z4250, Sigma-Aldrich) was injected into the plantar side of a hind paw. Polychromatic flow cytometry analysis was performed as describes above. Cells were stained with CD117-PE PE (Miltenyi, \#130-102-795), FcERla-FITC (\#134305, Biolegend), Ly6C-eFluor450 (eBioscience \#48-5932-82) Ly6G/Ly6C-FITC (eBioscience, \#11-5931-82), F4/80-PE/Cy7 (Biolegend, \#123113), F4/80-PE (Biolegend, \#123110), Siglec F-PE-Vio770 (Miltenyi, \#130-102-167), CD45-VioGreen (Miltenyi, \#130-123-900), CD206-APC (Biolegend, \#141708), CD86-BrilliantViolet421 (Biolegend, \#105031), CD11b-APC/Cy7 (Biolegend, \#101225), MHC II-FITC (Miltenyi, 130-081-601). Macrophages were identified as

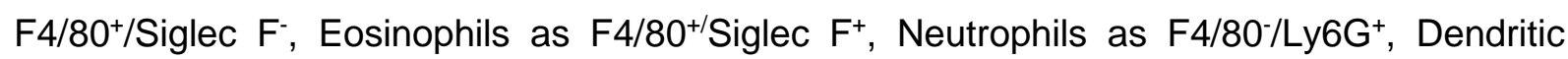
Cells as F4/80-/Ly6G-/CD11 b/MHC II+. M1 Macrophages were identified as F4/80+/CD86 ${ }^{+}$, M2 Macrophages as $\mathrm{F} 4 / 80^{+} / \mathrm{CD} 206^{+}$. The gating strategy is shown in figure 7 . Mast cells were identified as $\mathrm{CD} 117^{+} \mathrm{Fc \varepsilon Rl \alpha ^{+ }}$; flow plots and gating strategy are shown alongside the quantification in figure 16.

For analysis of phagocytosis, Mcpt5-DTA Cre- and $\mathrm{Cre}^{+}$mice were injected with $10 \mu \mathrm{l}$ pHrodo-zymosan (12 mg/ml in PBS, 1:1 mix of unlabeled zymosan (Sigma-Aldrich, \#Z4250,) and pHrodo $^{\mathrm{TM}}$ Red Zymosan Bioparticles ${ }^{\mathrm{TM}}$ (Invitrogen, \#P35364,)) per paw. pHrodozymosan within macrophages or neutrophils was detected in the B2 channel of the FACS. Macrophages were identified as $\mathrm{F} 4 / 80^{+}$, Neutrophils as $\mathrm{F} 4 / 80^{-} / \mathrm{Ly} 6 \mathrm{G}^{+}$. For analysis of phagocytosed neutrophils, intracellular staining with Ly6G-APC (Biolegend, \#127614) was performed. Cells were prepared as described above, incubated with ACK buffer, fixed for 5 min with $1 \%$ PFA and washed with PBS. Then the cells were permeabilized with $0.1 \%$ Saponin and incubated for $1 \mathrm{~h}$ at $4^{\circ} \mathrm{C}$ with antibodies in FACS buffer containing $0.1 \%$ Saponin. After incubation, the cells were washed with $0.1 \%$ Saponin and resuspended in PBS for flow cytometry. Flow plots are shown alongside the quantification in figure 19.

\subsubsection{Primary cell culture and in vitro experiments}

2.2.3.1. Isolation and cultivation bone marrow-derived mast cells (BMMC)

Bone marrow-derived mast cells were prepared as described previously (Kornstädt et al., 2021; Tarighi et al., 2019; Treutlein et al., 2018). Bone marrow cells were isolated from murine femur and tibia from the hind legs of adult mice. The bones were cut open at one end and centrifuged into $100 \mu \mathrm{l}$ mast cell medium at $10,000 \mathrm{~g}$ for $1 \mathrm{~min}$. The cells of a single animal were then suspended in $40 \mathrm{ml}$ of mast cell medium consisting of RPMI 1640 medium 
supplemented with $10 \%$ fetal bovine serum, $100 \mathrm{U} / \mathrm{ml}$ penicillin/streptomycin, $4 \mathrm{mM} \mathrm{L-}$ Glutamine, $1 \mathrm{mM}$ sodium pyruvate, 1\% MEM non-essential amino acids, $50 \mu \mathrm{M} 2-$ mercaptoethanol and $10 \mu \mathrm{g} /$ liter IL-3. $40 \mathrm{ml}$ of medium was added twice a week. During the first two weeks, the flasks were exchanged to remove adherent cells. Cells were kept at 37 ${ }^{\circ} \mathrm{C}$ at $5 \% \mathrm{CO}_{2}$. Bone marrow-derived mast cell cultures were used for experiments after 4 to 6 weeks of cultivation.

After 4 to 6 weeks, the purity and maturity of the mast cells was assessed by polychromatic flow cytometry on a FACS Canto II flow cytometer (BD Life Sciences). Therefore, $50 \mathrm{ml}$ of the cell suspension was transferred to a $50 \mathrm{ml}$ Falcon tube and centrifuged at $500 \mathrm{~g}$ for 10 minutes. The supernatant was discarded and the residual cells were resuspended in $200 \mu \mathrm{l}$ PBS and transferred to a 96-well V-bottom plate for washing. Cells were then centrifuged at $500 \mathrm{~g}$ for $10 \mathrm{~min}$ again, supernatant was discarded and the pellet was resuspended in $50 \mu \mathrm{l}$ antibody mastermix in FACS buffer (1\% FBS in PBS) containing CD117-PE (Miltenyi, \#130102-795) and FceRl $\alpha$-FITC (Biolegend, \#134305) and incubated for 1 hour at $4^{\circ} \mathrm{C}$ in the dark. The labelled cells were then washed once with FACS buffer, resuspended in PBS and analysed on a BD FACS Canto II (BD Life Sciences). FlowJo software V10.4 (both BD Life Sciences) was used for analysis and for gating, unstained controls and fluorescence minus one (FMO) controls were used.

\subsubsection{Isolation and cultivation bone marrow-derived macrophages (BMDM)}

For generation of bone marrow-derived macrophages, bone marrow cells were isolated from murine femur and tibia from the hind legs of adult mice as described previously (Kern et al., 2018; Kornstädt et al., 2021; Pierre et al., 2017). The bones were cut open at one end and centrifuged into $100 \mu \mathrm{l}$ macrophage medium at $10,000 \mathrm{~g}$ for $1 \mathrm{~min}$. The cells of a single animal were then suspended in $12 \mathrm{ml}$ macrophage medium consisting of RPMI1640 GlutaMAX medium supplemented with $10 \%$ fetal bovine serum, $100 \mathrm{U} / \mathrm{ml}$ penicillin/streptomycin, and $20 \mathrm{ng} / \mathrm{ml}$ macrophage colony stimulating factor (M-CSF) to initiate macrophage maturation. Cells were seeded into 6 well plate and cultivated in $2 \mathrm{ml}$ medium. Cells were kept at $37^{\circ} \mathrm{C}$ at $5 \% \mathrm{CO}_{2}$. Non-adherent cells were removed after one day of cultivation by exchange of medium. $2 \mathrm{ml}$ of fresh medium were added after 4 days and differentiation towards mature macrophages was completed after 7 days. 


\subsubsection{In vitro experiments with BMMCs and BMDMs}

BMDMs and BMMCs were differentiated as described. Cell number of BMMCs was adjusted to $8 \times 10^{5}$ cells $/ \mathrm{ml}$ in $2 \mathrm{ml}$ final volume by centrifugation (500 g, $10 \mathrm{~min}$ ), followed by adjusting the concentration by addition of mast cell medium. BMMCs and BMDMs were treated as indicated in the respective figure legends with $10 \mu \mathrm{g} / \mathrm{ml}$ zymosan (Sigma-Aldrich, \#Z4250), $100 \mathrm{ng} / \mathrm{ml}$ LPS (Sigma-Aldrich, \#L3129), 30 ng IL-13 (PeproTech, \#213-13-100UG), $5 \mu \mathrm{M}$ S1P (Cayman Chemicals, \#62570), 100 ng/ml LysoPC, $25 \mu \mathrm{M}$ Cytochalasin D (SigmaAldrich, \#C2618), $50 \mu \mathrm{M}$ Bafilomycin A (Sigma-Aldrich, \#SML1661), $10 \mu \mathrm{M}$ MEK inhibitor U0126 (Cell Signaling Technology, \#9903), 10 MM NOD2 inhibitor GSK 717 (Calbiochem, \#533718), or $10 \mu \mathrm{M}$ PAFR inhibitor WEB 2086 (Tocris, \#2339).

For analysis of mediator release by degranulation, BMMCs suitable for experiments were centrifuged at $500 \mathrm{~g}$ for $10 \mathrm{~min}$, counted and adjusted to a concentration of $8 \times 10^{5} \mathrm{cells} / \mathrm{ml}$. Cells intended for subsequent measurement of histamine release were resuspended in $1 \mathrm{ml}$ Tyrode's buffer per condition, cells intended for measurement of cytokine release upon degranulation were resuspended in $2 \mathrm{ml}$ mast cell medium per condition. BMMCs were then pre-incubated with $1 \mu \mathrm{g} / \mathrm{ml}$ DNP-specific IgE (Sigma-Aldrich, \#D8406) for 1 hour and then washed by adding twice the volume of the respective medium and centrifuging them at $500 \mathrm{~g}$ for $10 \mathrm{~min}$. They were then resuspended in Tyrode's buffer or mast cell medium, depending on the intended usage. Then BMCs were induced with $100 \mathrm{ng} / \mathrm{ml}$ DNP-BSA Conjugate (Alpha Diagnostics, \#DNP35-N-10) for 5 min for histamine measurement and for 24 hours for cytokine measurement.

Supernatant and cell pellet were stored at $-80^{\circ} \mathrm{C}$ until measurement. Cytokines were measured by ELISA according to the manufacturer's instruction, prostanoids and histamine were measured by LC-MS/MS as described in 2.2.4. Bionalytical methods.

\subsubsection{Bioanalytical methods}

\subsubsection{Multiplex Cytokine Assay}

Levels of 23 cytokines and chemokines in ipsi- and contralateral paws of Mcpt5-DTA mice were determined using the Bio-Plex Pro mouse cytokine group I (Bio-Rad) as described in Kornstädt et al. (2021). Perfect pairs of Mcpt5-DTA Cre ${ }^{+}$and Cre- animals were injected with $10 \mu \mathrm{l}$ of $12 \mathrm{mg} / \mathrm{ml}$ Zymosan in one hind paw, the other paw served as the contralateral control. Animals were killed 48 hours after the injection of zymosan into the paw and the paw 
tissue was then dissected and frozen at $-80^{\circ} \mathrm{C}$ until the protein extraction. For the protein extraction, the paw tissue was suspended in $400 \mu$ lysis buffer each, consisting of Tissue Extraction buffer (Invitrogen, \#FNN0071) and protease inhibitor (Roche, \#11697498001). The samples were cut into small pieces and then sonicated two times at $60 \%$ power for 10 sec. Afterwards all samples were centrifuged at 10,000 rpm for 5 min to pellet tissue debris. The concentration of total protein in the samples was assessed by bicinchoninic acid assay. All samples were diluted 1:20 to a final protein concentration of $200-900 \mu \mathrm{g} / \mathrm{ml}$, according to the kit requirements.

For comparison of cytokines and chemokines in the plasma of mice injected with $10 \mu \mathrm{l}$ zymosan $(3 \mathrm{mg} / \mathrm{ml})$ or LPS $(100 \mu \mathrm{g} / \mathrm{ml})$ into the paw, $300 \mu \mathrm{l}$ blood was obtained by cardial punctuation 5 hours after intraplantar injection and further diluted with $30 \mu \mathrm{l}$ citrate and $500 \mu \mathrm{l}$ DPBS (w/o Ca2+, $\mathrm{Mg}^{+}$) and 10\% acid-citrate-dextrose. The blood was centrifuged at $3000 \mathrm{~g}$ for $15 \mathrm{~min}$ at room temperature. Plasma was collected, aliquoted and frozen at $-80^{\circ} \mathrm{C}$. Levels of 25 cytokines and chemokines were determined in $25 \mu \mathrm{l}$ plasma using the Mouse Cytokine/Chemokine beads immunoassay kit (ProcartaPlex Multiplex Immunoassay, Thermofisher) as described in Hahnefeld \& Kornstädt et al. (2021). Comparison of cytokines and chemokines in the plasma of zymosan- and LPS-injected mice was performed by Sandra Pierre.

Concentrations of cytokines and chemokines were measured with a Bioplex 200 (Bio-Rad) according to the manufacturer's instruction and normalized to the total protein concentration of the respective sample.

\subsubsection{LC-MS/MS analysis of prostanoids}

LC-MS/MS analysis of $\mathrm{PGE}_{2}$ in BMMC culture medium was performed as described previously (Kornstädt et al. 2021; Treutlein et al., 2018). Briefly, $100 \mu \mathrm{l} \mathrm{PBS,} 100 \mu \mathrm{l} 150 \mathrm{mM}$ EDTA and $20 \mu$ internal standard solution (10 ng/ml of $\left[{ }^{2} \mathrm{H}_{4}\right]-\mathrm{PGE}_{2}$ in methanol) were added to $100 \mu \mathrm{l}$ cell suspension before extraction liquid-liquid extraction using with $600 \mu \mathrm{l}$ ethyl acetate. Organic layer was separated and the extraction was repeated using again $600 \mu \mathrm{l}$ ethyl acetate. The organic layers were combined, evaporated at $45^{\circ} \mathrm{C}$ under a gentle stream of nitrogen and reconstituted with $50 \mu$ of acetonitrile:water:formic acid (20:80:0.0025, v/v). $10 \mu \mathrm{l}$ of this solution were injected into the LC-MS/MS system. For LC-MS/MS analysis an Agilent 1290 Infinity LC system (Agilent, Waldbronn, Germany) coupled to a hybrid triple quadrupole linear ion trap mass spectrometer QTRAP 6500+ (Sciex, Darmstadt, Germany) equipped with a Turbo-V-source operating in negative ESI mode was used. Chromatographic separation was done using a Synergi Hydro-RP column $(2.0$ x 150 mm, $4 \mu \mathrm{m}$ particle size; 
Phenomenex, Aschaffenburg, Germany), coupled to a precolumn of the same material. $0.0025 \%$ formic acid and acetonitrile containing $0.0025 \%$ formic acid served as mobile phases. Mass spectrometric parameters were: lonspray-voltage $4500 \mathrm{~V}$, source temperature $500^{\circ} \mathrm{C}$, curtain-gas 40 psi, nebulizer-gas 40 psi, Turboheater-gas 60 psi. Both quadrupoles were running at unit resolution. For analysis, Analyst Software 1.6 and Multiquant Software 3.0 (both Sciex, Darmstadt, Germany) were used, employing the internal standard method (isotope-dilution mass spectrometry).

LC-MS/MS analysis of prostanoids was performed by Yannick Schreiber and Dominique Thomas.

2.2.4.3. LC-MS/MS analysis of histamine

Histamine quantification was performed using a LC-MS/MS system consisted of a triple quadrupole tandem mass spectrometer QTRAP 5500 (Sciex, Darmstadt, Germany) equipped with a Turbo- $V$ source operating in positive electrospray ionization mode with an electrospray voltage of $5000 \mathrm{~V}$ at $450^{\circ} \mathrm{C}$, an Agilent 1200 binary HPLC pump and degasser (Agilent, Waldbronn, Germany), and an HTC Pal autosampler (CTC analytics, Zwingen, Switzerland). High-purity nitrogen for the mass spectrometer was produced by a NGM $22-$ LC-MS nitrogen generator (cmc Instruments, Eschborn, Germany).

Cell culture supernatants or cell culture media samples were spiked with $20 \mu \mathrm{L}$ of the internal standard solution (histamine-D4, $12 \mathrm{ng} / \mathrm{mL}$ in ethanol) and diluted with $20 \mu \mathrm{L}$ ethanol (as replacement of the standard solution) and $125 \mu \mathrm{L}$ of mobile phase B. Samples were vortexed, centrifuged for $2 \mathrm{~min}$ at $20000^{\prime} \mathrm{g}$ and transferred to a 96-well plate. $2.5 \mu \mathrm{L}$ were injected into the LC system. Multiple reaction monitoring (MRM) was used for identification and quantification. The mass transition used for histamine quantification was $\mathrm{m} / \mathrm{z}$ $112.1 \rightarrow 94.9$ (declustering potential, DP, $60 \mathrm{~V}$, collision energy, CE, $21 \mathrm{~V}$ ) with a dwell time of $20 \mathrm{~ms}$. For the chromatographic separation of histamine, a Acquity UPLC BEH Amide column (50 x $2.1 \mathrm{~mm}$ inner diameter, $1.7 \mu \mathrm{m}$ particle size, Eschborn, Germany, Waters) and a precolumn UHPLC C8 (2.1 mm, Phenomenex, Aschaffenburg, Germany) were used. A linear gradient was run at a flow rate of $0.6 \mathrm{~mL} / \mathrm{min}$ with a total run time of $7 \mathrm{~min}$. Mobile phase $A$ consist of ammonium formate $10 \mathrm{mM}$ and mobile phase $B$ of ammonium formate 10 $\mathrm{mM}$ and formic acid $10 \mathrm{mM}$ in acetonitrile containing $5 \%$ of mobile phase A. The gradient started with $100 \%$ B for 0.25 min. It changed to $20 \%$ B in 2.25 min and was maintained for 1 more $\min$ at $20 \% \mathrm{~B}$. It returned to $100 \% \mathrm{~B}$ in $0.1 \mathrm{~min}$ and maintained for $0.9 \mathrm{~min}$ at these conditions. Then, the flow was increased to $1 \mathrm{~mL} / \mathrm{min}$ in $0.1 \mathrm{~min}$ and after $0.9 \mathrm{~min}$, returned to $0,6 \mathrm{~mL} / \mathrm{min}$ until the end of the run time. Quantification was performed with Analyst 
software version 1.6.2 (Sciex) and Multiquant software version 3.0.2 (Sciex) using the internal standard method (isotope-dilution mass spectrometry). Ratios of analyte peak area and internal standard area (y-axis) were plotted against concentration ( $x$-axis), and calibration curves were calculated by least-linear regression.

LC-MS/MS analysis of histamine was performed by Yannick Schreiber and Dominique Thomas.

\subsubsection{LC-QTOFMS analysis of lipids}

Sample preparation and LC-QTOFMS analysis was performed as previously published (Hahnefeld et al., 2019; Hahnefeld \& Kornstädt et al., 2021). Briefly, lipids were extracted from $20 \mu \mathrm{L}$ of mouse plasma using $150 \mu \mathrm{L}$ of internal standard solution in methanol, $500 \mu \mathrm{L}$ MTBE and $125 \mu \mathrm{L} 50 \mathrm{mM}$ ammonium formate. The upper organic phase was transferred and the aqueous phase re-extracted with $200 \mu \mathrm{L}$ of a mixture of MTBE: methanol: water (10:3:2.5, v/v/v, upper phase). The combined organic phases were then split in to two $290 \mu \mathrm{L}$ aliquots for measurement in positive and negative ionization mode and dried under a nitrogen stream at $45{ }^{\circ} \mathrm{C}$. Prior analysis, the residues were reconstituted in $120 \mu \mathrm{L}$ of methanol.

Paws were weighed in a $2 \mathrm{~mL}$ reinforced tube. After addition of five zirconium dioxide grinding seeds (2.8 mm diameter), tissue concentration was adjusted to $0.05 \mathrm{mg} / \mu \mathrm{L}$ with ethanol: water $(1: 3, v / v)$. Shredding was performed on a Precellys 24 tissue homogenizer (Bertin Instruments, Montigny-le-Bretonneux, France) with $6500 \mathrm{rpm}$ for 2x45 s and dry ice cooling. $20 \mu \mathrm{L}$ of the resulting homogenate were extracted as described above for plasma samples. Quality control samples were obtained from a pool of 24 homogenates.

LC-MS analysis was conducted on Nexera X2 system (Shimadzu Corporation, Kyoto, Japan) coupled to a TripleTOF 6600 (Sciex, Darmstadt, Germany) using electrospray ionization. Chromatographic separation was achieved using a Zorbax RRHD Eclipse Plus C8 $1.8 \mu \mathrm{m}$ $50 \times 2.1 \mathrm{~mm}$ ID column (Agilent, Waldbronn, Germany) and a 17 min linear gradient with a flow rate of $0.3 \mathrm{~mL} / \mathrm{min}$. The mobile phases were $A 10 \mathrm{mM}$ ammonium formate and $0.1 \%$ formic acid in water for positive and $1 \mathrm{mM}$ ammonium formate and $0.1 \%$ formic acid in water for negative ionization mode as well as B $0.1 \%$ formic acid in acetonitrile: isopropanol (2:3, $\mathrm{v} / \mathrm{v}$ ) for both ionization modes. The TOF-MS Scan covered a mass range from 100 to 1000 $\mathrm{m} / \mathrm{z}$. Six data dependent spectra per cycle with a mass range from 50-1000 m/z and a CE of +/- $40 \mathrm{~V}$ with a $20 \mathrm{~V}$ collision energy spread and dynamic background subtraction allowed for better identification of the analytes. 
QTOF-MS spectra were acquired using Analyst TF v1.7.1 and peak alignment and filtering was done using MarkerView software v1.2.1 with a mass and retention time tolerance of 10 ppm and $0.15 \mathrm{~min}$, respectively. The samples were normalized using multiple internal standards for positive and negative ionization mode. Features were excluded based on their identification as isotope, a coefficient of variation for the quality control samples exceeding $20 \%$ or if less than $80 \%$ of the samples had a greater peak area than the extracted blank. Combination of the positive and negative ionization mode resulted in 4150 features in plasma and 2445 features in paw samples. Significantly changed lipids were identified using exact mass with a $5 \mathrm{ppm}$ tolerance, isotopic distribution and via data-dependent spectra, which were cross-checked with LIPID MAPS (http://www.lipidmaps.org), METLIN (http://metlin.scripps.edu) and the Human Metabolome Database (HMDB, version 4.0). The peak areas of the identified lipids were calculated using Multiquant 3.02 software and normalized with one internal standard per lipid class. All data acquisition and processing software was purchased from Sciex (Darmstadt, Germany).

LC-QTOFMS analysis of lipids was performed by Lisa Hahnefeld.

\subsubsection{RNA Sequencing for differential expression analysis}

RNA sequencing and differential expression analysis was performed as published in Kornstädt et al. (2021) and is described here in detail.

\subsubsection{Sample Preparation and RNA sequencing}

Cells were isolated from the bone marrow of C57BL/6N mice and differentiated into bone marrow-derived mast cells as described in 2.2.3. Primary cell culture and in vitro experiments. After 4 weeks of cultivation, the cell number was adjusted to $8 \times 10^{5} \mathrm{cells} / \mathrm{ml}$, the cells were seeded into cell culture dishes and induced with $10 \mu \mathrm{g} / \mathrm{ml}$ zymosan for 24 hours or 48 hours or left untreated as 0 hours control.

After the incubation time with zymosan, the cells were prepared for fluorescence-activated cell sorting (FACS). Therefore, the cells were transferred into $15 \mathrm{ml}$ Falcon tubes and centrifuged at $500 \mathrm{~g}$ for $10 \mathrm{~min}$. The supernatant was taken, aliquoted and stored at $-80^{\circ} \mathrm{C}$ for subsequent analysis by ELISA and residual cells were resuspended in $120 \mu$ PBS for washing and transferred to a 96 well V-bottom plate. Cells were centrifuged at $500 \mathrm{~g}$ for 10 min again, the supernatant was discarded and residual cells were resuspended in $30 \mu \mathrm{l}$ FACS buffer (1\% FBS in PBS). Then, $20 \mu \mathrm{l}$ of antibody mastermix containing CD117-PE 
(Miltenyi, \#130-102-795) and anti-mouse FceRl $\alpha$-FITC (Biolegend, \#134305) was added and the cells were incubated for 1 hour at $4^{\circ} \mathrm{C}$ for staining of surface molecules. After the staining, the cells were washed with FACS buffer once, resuspended in PBS and transferred through $70 \mu \mathrm{m}$ cell strainers to FACS tubes for subsequent cell sorting. Samples were kept in the dark and on ice until sorting. About 1 million BMMCs per condition were sorted into $5 \mathrm{ml}$ PBS as $\mathrm{CD} 117^{+} / \mathrm{FC} \varepsilon \mathrm{Rl} \alpha^{+}$using a FACS Aria sorter.

A: P1

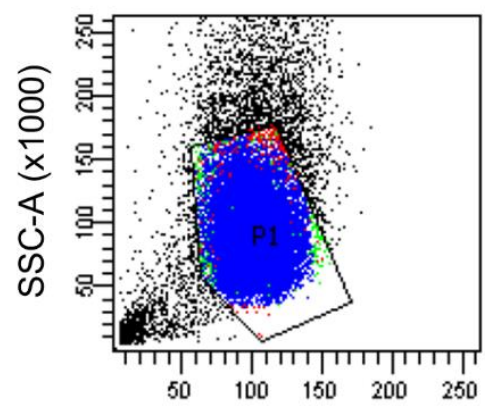

FSC-H (x1000)

C: $\mathrm{P3}$

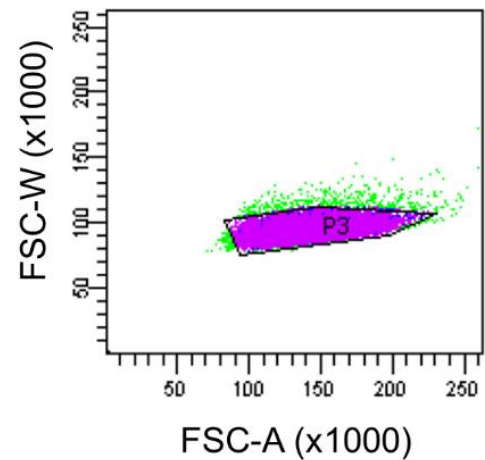

B: P2

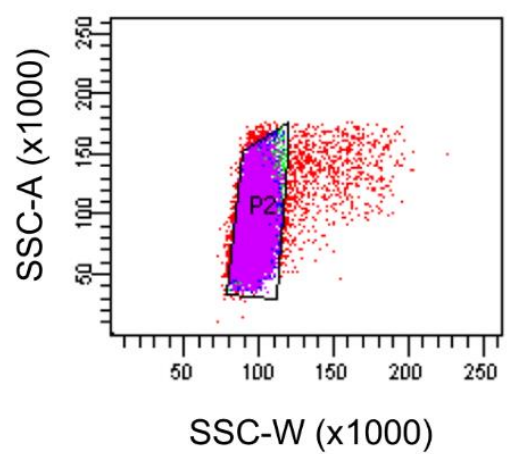

D: P4

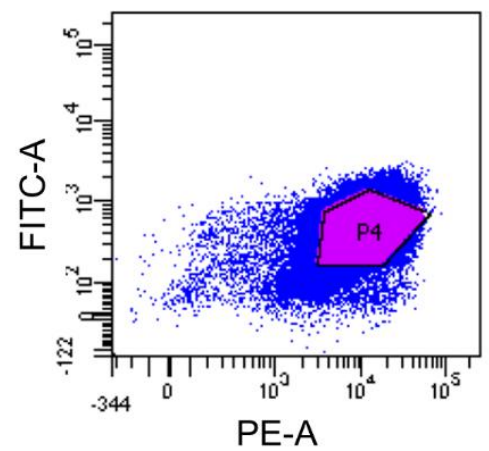

E

\begin{tabular}{|c|c|c|c|}
\hline Population & \#Events & \%Parent & \%Total \\
\hline All Events & 100,000 & \#\# & 100.0 \\
\hline$\square \mathrm{P} 1$ & 79,829 & 79.8 & 79.8 \\
\hline & 74,194 & 92.9 & 74.2 \\
\hline & 70,551 & 95.1 & 70.6 \\
\hline & 55,047 & 78.0 & 55.0 \\
\hline
\end{tabular}

Figure 8: Gating strategy for FACS sorting of mature BMMCs for RNA sequencing. Reprinted from Kornstädt et al. (2021). (A) Cells were gated based on their side scatter area (SSC-A) and forward scatter height (FSC-H) in P1. (B) Single cells were then identified within P1 based on their side scatter area (SSC-A) and side scatter width (SSC-W) in P2, followed by (C) their forward scatter width (FSC-W) and forward scatter area (FSCA) in P3. (D) Mast cells were identified within P3 as CD117 ${ }^{+} F c \varepsilon R l \alpha^{+}$cells. (E) Exemplary data of percentage of mast cells (P4) sorted for subsequent RNA sequencing. 
RNA from BMMCs was extracted using the RNeasy Micro Kit (Qiagen), following the manufacturer's instructions. Briefly, BMMCs obtained from FACS sorting were collected by centrifugation at $500 \mathrm{~g}$ for 10 min directly after FACS and immediately resuspended in denaturing lysis buffer, which disrupts the plasma membrane and inactivates RNases to prevent RNA degradation. The cells were then homogenized by vortexing for $1 \mathrm{~min}$, followed by addition of an equal volume of $70 \%$ ethanol, which is both required for efficient binding of RNA to the RNeasy MinElute spin column (Qiagen). After disruption, homogenization and addition of ethanol, the sample is then transferred to a spin column. DNA is removed by DNase treatment and DNase and other remaining contaminants are subsequently washed away, while RNA stays bound to the silica-based membrane of the spin column. Finally, RNA is eluted in RNase-free water and stored at $-80^{\circ} \mathrm{C}$ until further usage.

Quality and quantity of the RNA samples was evaluated on an Agilent 2100 Bioanalyzer using an RNA 6000 Pico Chip (Agilent), additional to quantification on a NanoDrop (ThermoFisher). Around $100 \mathrm{ng}$ of RNA per sample was taken for library preparation using the QuantSeq 3' mRNA Library Prep Kit FWD (Lexogen) according to manufacturer's instructions. Briefly, first strand cDNA synthesis is performed by reverse transcription with an oligodT Primer containing an Illumina-compatible sequence at its 5' end. Then, the template RNA is degraded and second strand synthesis with random primers containing an Illuminacompatible linker sequence at its 5' end converts the library to dsDNA. Reaction components are then removed by purification with magnetic beads. Finally, complete adapter sequences for cluster generation and unique indices for multiplexing are added and the libraries are amplified to generate sufficient material for quality control and sequencing. Optimal PCR cycle number for Library Amplification was evaluated by qPCR. In a final step, PCR components are removed from the libraries by purification wit magnetic beads.

The Quality of the resulting cDNA libraries was assessed with an Agilent High Sensitivity DNA Chip (Agilent), quantity was evaluated by a Qubit ds DNA HS assay (Thermo Fisher). The libraries are then normalized and pooled to a concentration of $1 \mathrm{nM}$. Then, libraries are denatured and diluted to a final concentration of $2.2 \mathrm{pM}$ following Illumina and Lexogen instructions and loaded onto the reagent catridge of the High Output Kit v2 (Illumina, singleend). Sequencing was performed on a NextSeq 500 (Illumina).

\subsubsection{Analysis of RNA sequencing data}

RNA sequencing data was analysed by Stefanie Ebersberger and Ingo Ebersberger. The data are available in the GEO database with the accession number GSE165523. 


\section{Preprocessing}

The primary assembly of the mouse genome sequence as of June 2018 (GRCm38.p6) was used as a reference genome. The data was downloaded from the Gencode website (ftp://ftp.ebi.ac.uk/pub/databases/gencode/Gencode_mouse/release_M17/GRCm38.primary_ assembly.genome.fa.gz).

The genome annotation was downloaded from Gencode (Release M17, GRC038.p6. ftp://ftp.ebi.ac.uk/pub/databases/gencode/Gencode_mouse/release_M17/gencode.vM17.ann otation.gtf.gz).

The raw sequencing reads were preprocessed with the software bcl2fastq using the following command:

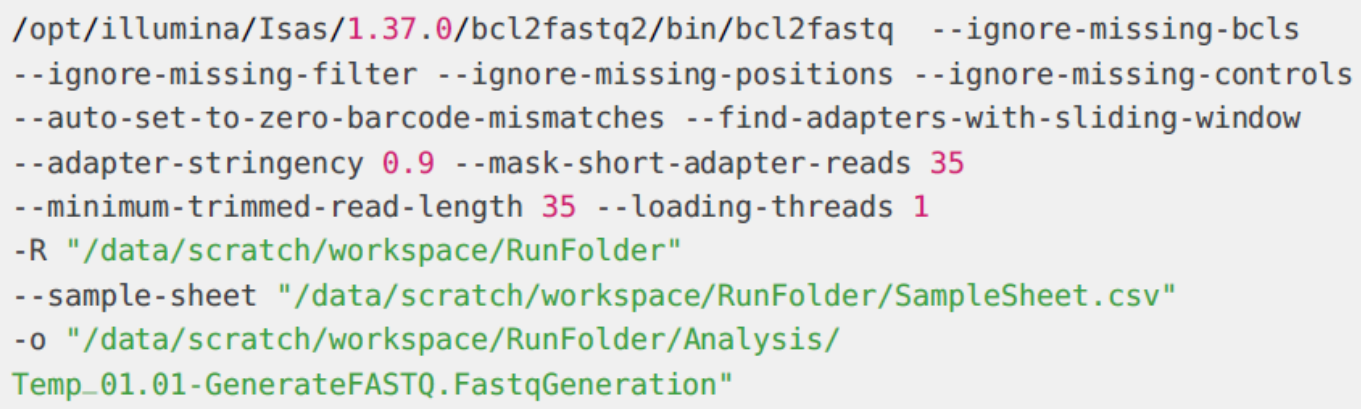

The four fastq files for each sample were concatenated, and summary statistics for the 12 samples were generated with FASTQC.

\section{Read mapping}

Read mapping against the mouse reference genome was done with the STAR aligner (version 2.6.0a 2018/04/23).

- Generation of the genome index

-runMode genomeGenerate -genomeDIR /share/project/ingo/projects/MLL-Inflammation/GRch38/Genomelndex -genomeFastaFiles GRCm38.p6.primary_assembly.genome.fa -sjdbGTFfile gencode.vm17.annotation.gtf -sjdbOverhang 74

- Read mapping

- runThreadN 15

-runMode alignReads

-genomeDir/share/project/ingo/projects/MLL-Inflammation/GRCm38/Genomelndex 
-readFilesIn 7001.fastq

-outStd SAM

-genomeLoad NoSharedMemory

-outSJfilterRads unique

-outSAMunmapped Within

-outReadsUnmapped none

-limitGenomeGenerateRAM 31000000000

-limitlObufferSize 150000000

-outFilterMismatchNmax 5

-outFilterMultimapNmax 10

-alignIntronMin 21

-sjdbOverhang 74

The average mapping result across the 12 samples was

- Unique mappers: $76 \%$

- Multimappers: $16 \%$

- Mapping to too many loci: $2 \%$

- Not mapping because too short: $5 \%$

- Annotation of mapped reads

The mapped reads were assigned to annotated features (genes) using the Bioconductor package Rsubread (v1.28.1). Unassignable reads comprise reads overlapping with multiple genes (ambigiuous), non-unique mappers or because of no overlap with known genes. On average $75 \%$ of uniquely mapped reads could be assigned to genes.

Diffrential expression analysis

Differential expression of genes between conditions was assessed with the Bioconductor package DESeq2 (v1.18.1).

- Data quality assessment

A principal component analysis (PCA) was done on the normalized (variance stabilizing transformation) data to assess the overall effect of experimental covariates and batch effects. The variance stabilizing transformation removes the dependence of the variance on the mean and therefore reduces the high variance of the logarithm of count data when the mean is low. The PCA is calculated on the 500 most variable genes across conditions. 
- Differentially expressed genes

Differentially expressed genes are identified by an adjusted $p$-value $<0.05$ and visualized in a MA plot, showing the mean of the normalized counts versus the log2 fold change of the genes.

\section{Gene ontology analysis}

Gene ontology analysis was performed with the gene set library "GO Biological Processes 2018" by Enrichr (Chen et al., 2013; Kuleshov et al., 2016). 


\section{Results}

3.1. Comparison of two locally induced inflammation models

Mast cells express members of the TLR family, enabling them to differentially recognize various pathogens. Both TLR2 and TLR4 are able to identify many different pathogens and they respond with partly overlapping signaling cascades. To study mast cells in the context of a local inflammation, we first aimed to characterize and compare the immune response evoked by two distinct pathogens commonly used to activate TLR2 and TLR4, namely zymosan and LPS. To induce a local inflammation zymosan or LPS was injected into the hind paw of mice.

\subsubsection{Mechanical hypersensitivity induced by LPS and zymosan}

Pain is one of five symptoms of an acute inflammation and is a useful indicator for the duration and intensity of the inflammation. Mechanical hypersensitivity describes the increased sensitivity to an innocuous stimulus caused by a sensitization of nociceptors by inflammatory mediators released at the site of inflammation. To compare the effect of zymosan and LPS it is useful to choose concentrations that evoke an inflammation of similar duration and intensity.

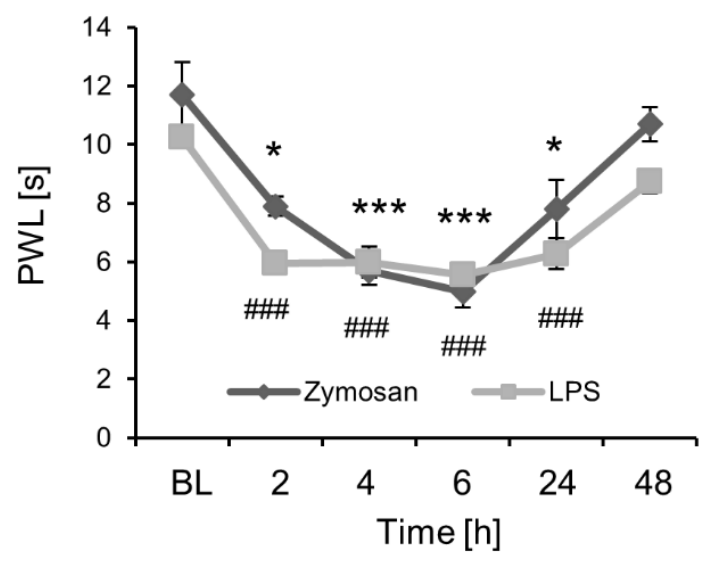

Figure 9: Comparison of mechanical hypersensitivity induced by LPS and zymosan. Reprinted from Hahnefeld \& Kornstädt et al. (2021). Paw withdrawal latencies (PWL) were measured at the indicated time points after injection of $10 \mu \mathrm{l}$ Zymosan $(3 \mathrm{mg} / \mathrm{ml})$ or LPS $(100 \mu \mathrm{g} / \mathrm{ml})$. Data are presented as mean of 6 mice \pm S.E.M.. One-Way ANOVA Bonferroni post test ${ }^{*} p<0.01,{ }^{* * *}$,\#\#\# $p<0.0001$ for zymosan $\left({ }^{*}\right)$ or LPS (\#) vs baseline (BL). Two-Way ANOVA Bonferroni post test showed no significant differences between zymosan and LPS groups. 
Mice were injected with $1 \mu \mathrm{g}$ LPS in $10 \mu$ PBS and $30 \mu \mathrm{g}$ zymosan in $10 \mu$ l PBS in the plantar side of one hind paw and mechanical hypersensitivity was assessed by measuring the paw withdrawal latency after applying force to the paw with a steel rod. The injection of $1 \mu \mathrm{g}$ LPS in $10 \mu \mathrm{l}$ and $30 \mu \mathrm{g}$ zymosan in $10 \mu \mathrm{l}$ PBS induced comparable paw withdrawal latencies at the same time points, both with the shortest paw withdrawal latency of less than 5 seconds 4-6 hours after injection, an observable decrease in pawl withdrawal latency after 24 hours, and reaching of baseline level again after 48 hours (Figure 9). This suggests a peak of the inflammation after 4-6 hours and a resolution phase starting one day after the injection.

Since $30 \mu \mathrm{g}$ zymosan and $1 \mu \mathrm{g}$ LPS evoked a similar response regarding the mechanical hypersensitivity, these concentrations were chosen for comparison of the two inflammation models in the following experiments.

\subsubsection{Stronger local inflammation regarding edema formation and levels of recruited immune cells in zymosan model compared to LPS model}

To further characterize the inflammatory responses evoked by zymosan and LPS, edema formation and immune cell recruitment in the inflamed paws in both models were analysed. Next to pain, edema formation is another symptom of an inflammation, resulting from accumulating fluid and leukocytes due to increased vascular permeability. To compare the immune cell recruitment to the site of inflammation induced by zymosan or LPS, polychromatic flow cytometry analysis of naïve paws and inflamed paws 5 hours after pathogen-injection was performed.

While injection of zymosan and LPS caused a similar mechanical hypersensitivity, edema formation could only be observed after zymosan injection (Figure 10A). In accordance with this, about 2-3 times more immune cells $\left(\mathrm{CD} 45^{+}\right.$cells, CD45+/Ly6C+ cells; Figure 6; Figure $10 \mathrm{~B}, \mathrm{C})$ were recruited to the site of inflammation induced by zymosan compared to LPS. Especially the percentage of neutrophils (CD45 $/$ Ly6G-Gr1 ${ }^{+}$cells; Figure 6; Figure 10D) and monocyte-derived macrophages (CD45+/F4/80+/Ly6C+ cells; Figure 6; Figure 10E) were highly increased after injection of zymosan. The numbers of dendritic cells (CD45 $/$ CD $11 \mathrm{c}^{+}$) and eosinophils $\left(\mathrm{CD}_{4} 5^{+} /\right.$Siglec $\left.\mathrm{F}^{+}\right)$are not significantly altered in either model as compared to tissue from naïve mice (Figure 6; Figure 10F,G). Taken together the data show two distinguished immunological responses in the two inflammation models, with a generally stronger reaction towards zymosan regarding the number of recruited immune cells. This 
stronger response and infiltration of leukocytes is mirrored by the edema formation, which could only be induced by zymosan and not by LPS (Figure 10A).
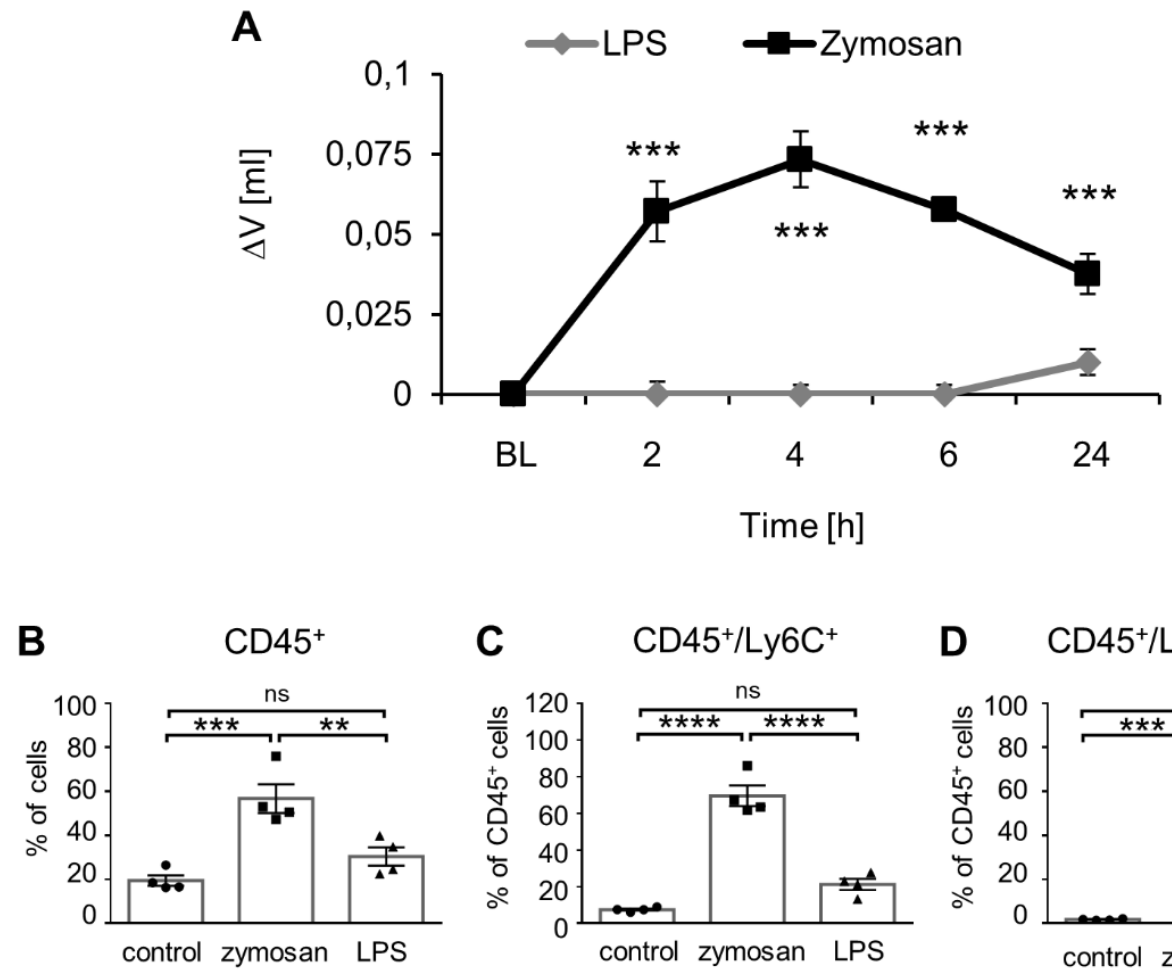

D $\quad$ CD45 $/$ Ly6G-GR1 ${ }^{+}$

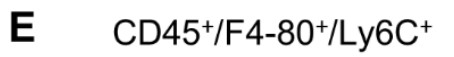

\section{F}

$\mathrm{CD} 45^{+} / \mathrm{CD} 11 \mathrm{c}^{+}$
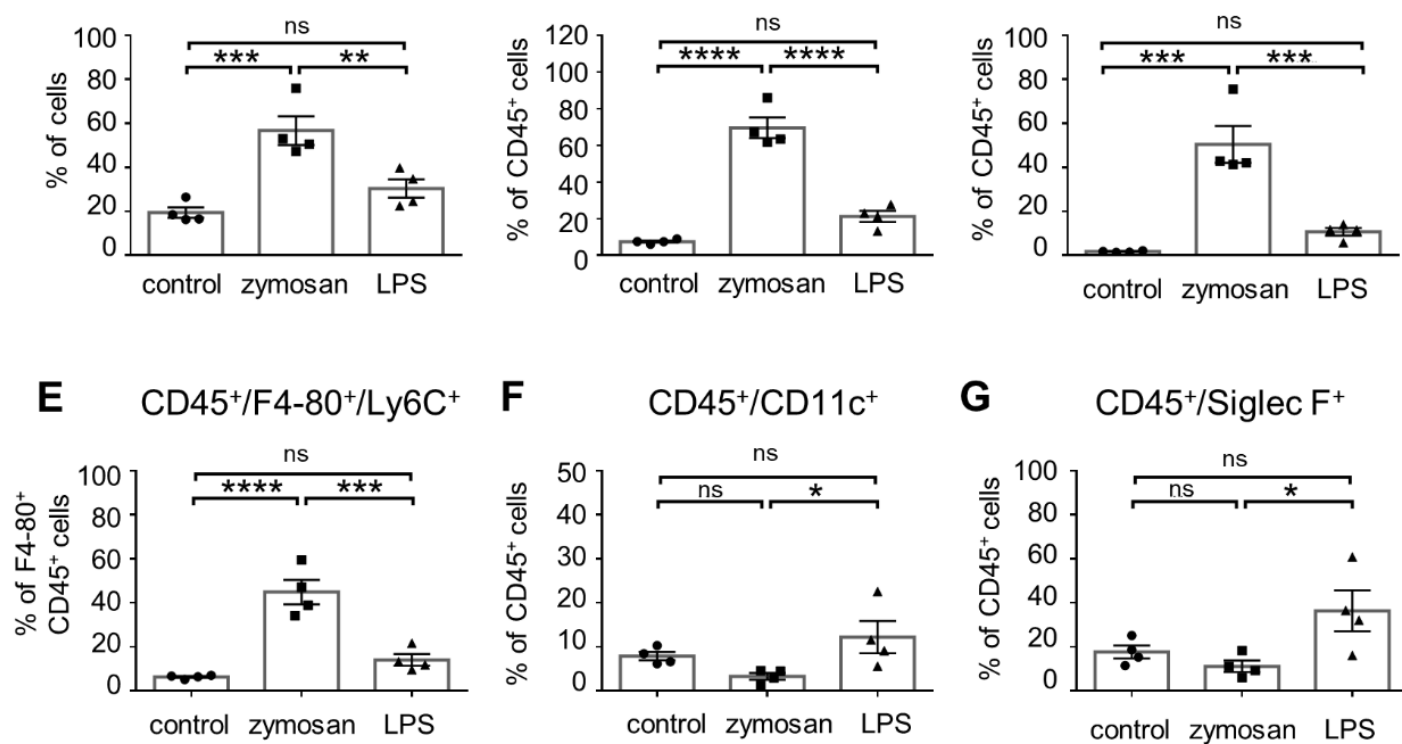

G $\quad \mathrm{CD} 45^{+} /$Siglec $\mathrm{F}^{+}$

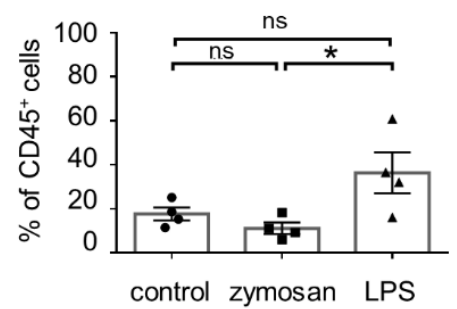

Figure 10: Edema formation and immune cell recruitment induced by LPS and zymosan. Figure reprinted from Hahnefeld \& Kornstädt et al. (2021). (A) Paw volume after injection of $10 \mu \mathrm{l} z y m o s a n(3 \mathrm{mg} / \mathrm{ml})$ or LPS $(100 \mu \mathrm{g} / \mathrm{ml})$ in hind paw of wild type mice at the indicated time point. Data are presented as mean \pm S.E.M. $(n=4)$. Two-way ANOVA/Bonferroni, ${ }^{* * *} \mathrm{p}<0.001$. (B-G) Flow cytometry analysis of immune cells in the paws of untreated mice or $5 \mathrm{~h}$ after injection of zymosan or LPS. Data are presented as mean \pm S.E.M. $(n=4)$. One-way ANOVA/Bonferroni, ${ }^{*} p<0.05,{ }^{* *} p<0.01,{ }^{* * *} p<0.001,{ }^{* * *} p<0.0001$.

\subsubsection{Lipid profiles and chemokine levels indicate a systemic immune response to} LPS and a local immune response to zymosan

Lipid mediators play a major role in the onset as well as in the resolution of inflammation. Lipid levels are strongly regulated in inflammatory processes and they have diverse functions 
in energy metabolism and as signaling molecules. To further identify differences and similarities between zymosan- and LPS-induced inflammation models, non-targeted lipidomic analysis was performed for the inflamed paw tissue 5 hours after pathogen injection.

Principal component analysis of the lipidomics experiment revealed distinct clusters for paws from naïve mice and paws from LPS- or zymosan-treated mice (Figure 11A). After LPSinjection 40 lipids were significantly regulated in the ipsilateral paws compared to naïve mice, while 13 lipids were significantly regulated after zymosan injection (Figure 11B). All of the lipids significantly regulated by zymosan were also significantly regulated by LPS and all of the significantly regulated lipids in the ipsilateral paws were upregulated. Among the lipids upregulated in both models are ether-phosphatidylcholines (PC O) and their corresponding lyso species (LPC O), which might have protective functions against reactive oxygen species typically released in inflammatory settings (Albert et al., 2001; Skaff et al., 2008). The most prominent lipids selectively released in the LPS-induced paws were lysophosphatidylcholines (LPC), which can be released by apoptotic cells, and steryl esters (SE), which play a role in cholesterol transport and have been reported to be part of the innate immune system (Lauber et al., 2003; Tall \& Yvan-Charvet, 2015). Comparison of the contralateral paws from zymosan- and LPS-injected mice with paws from naïve mice furthermore revealed that no lipids in the contralateral paws were significantly regulated by injection of zymosan. Meanwhile, 60 lipids were significantly regulated in the contralateral paws of LPS-injected mice. Interestingly, most of these lipids were uniquely regulated in the contralateral paw and only 8 of them were also significantly regulated in the ipsilateral paw (Figure 11C), suggesting mechanistically distinct responses in the ipsi- and contralateral paws. The most prominent lipids selectively regulated on the contralateral side are hydroxylated fatty acids (FA), phosphatidylcholines (PC), as well as phosphatidyl-ethanolamines (PE) and ether phosphatidylethanolamines (PE O). They all have been shown to be involved in oxidative phosphorylation and their increase might therefore correspond with an increased energy production due to inflammatory mechanisms (Calzada et al., 2019; Guo et al., 2017; Lange et al., 2001).

Lipid profiles of the plasma of naïve mice or pathogen-injected mice surprisingly showed that only one lipid was changed in the plasma after LPS injection, while 34 lipids were significantly altered in the plasma after injection of zymosan. The most prominent changes here were a decrease in fatty acids, diglycerides and triglycerides (data not shown here, published in Hahnefeld \& Kornstädt et al., 2021). This decrease of lipid levels in the plasma after zymosan injection into the paw can be explained by the energy required by the immune cells involved in the local inflammation. Meanwhile the absence of major changes in the lipid levels in LPS-injected mice is intriguing, considering the changes in the lipid levels in the contralateral paw in the LPS model. 
A

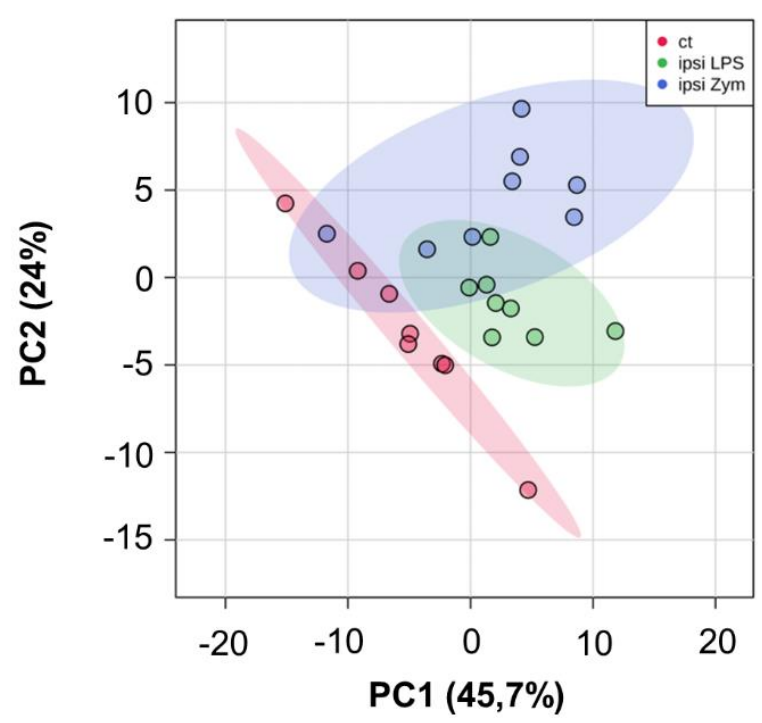

C
LPS

contralateral 60 regulated lipids
B

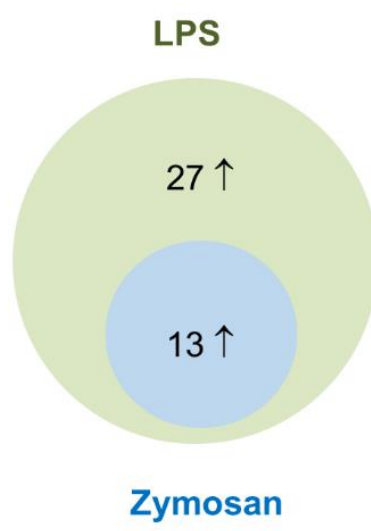

D

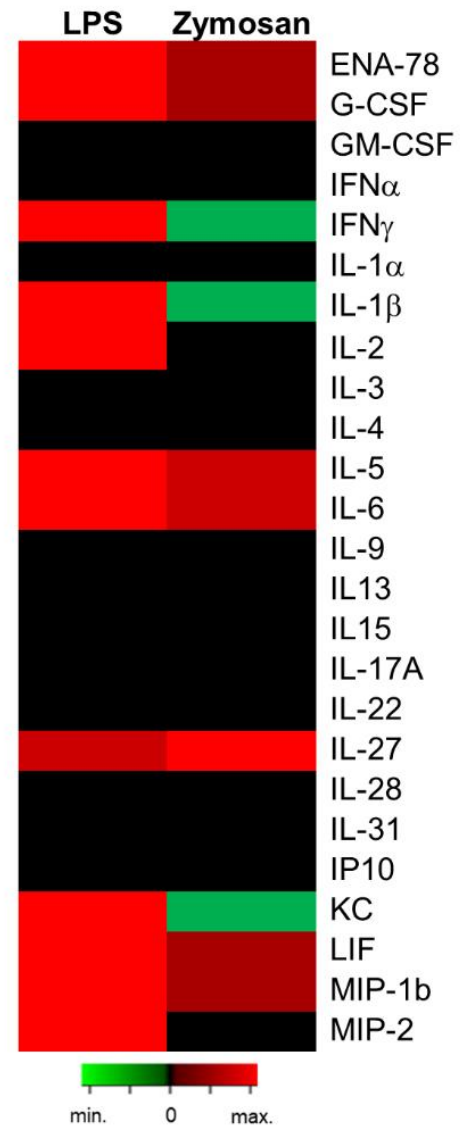

Figure 11: Distinct lipid profiles and chemokine levels after injection of zymosan or LPS. Figure adapted from Hahnefeld \& Kornstädt et al. (2021), analysis performed by Lisa Hahnefeld and Sandra Pierre. (A) Principal component (PC) analysis in ipsilateral paw samples (ipsi) shows differences in lipid profiles $5 \mathrm{~h}$ after injection of $10 \mu \mathrm{l}$ zymosan $(3 \mathrm{mg} / \mathrm{ml})$ or LPS $(100 \mu \mathrm{g} / \mathrm{ml})$ compared to untreated mice (95\% confidence interval, pareto scaling). (B) Number of significantly upregulated lipids in the injected paws after injection of LPS (green circle) or zymosan (blue circle) ( $n=8$, two-sided t-test with FDR correction). (C) Number of lipids significantly regulated in the ipsi- (green circle) and contralateral (red circle) paws $5 \mathrm{~h}$ after injection of LPS in comparison to paws of untreated mice $(n=8)$. (D) Heat map depicting change of 25 cytokines, chemokines and growth factors in the plasma of mice $5 \mathrm{~h}$ after LPS $(100 \mu \mathrm{g} / \mathrm{ml}, 10 \mu \mathrm{l})$ or zymosan $(3 \mathrm{mg} / \mathrm{ml}, 10 \mu \mathrm{l})$ injection compared to naïve mice $(\mathrm{n}=8)$. Green indicates downregulation, red upregulation. 
In search for an explanation of the changes in the contralateral side, plasma levels of 25 cytokines, chemokines and growth factors were analysed next. Out of these 25 mediators, 8 were increased significantly in the plasma after LPS injection, namely G-CSF, IL-1 $\beta$, IL-2, IL5, IL-6, MIP-1b, and MIP-2. Meanwhile, none were increased significantly after zymosan injection (Figure 11D). These mediators increased in the plasma after LPS injection can lead to the activation of immune cells throughout the body, which could explain the observed alterations in the lipid levels of the contralateral paws in the LPS model.

Taken together, this data demonstrates that a local injection of LPS evokes a systemic response, while the inflammation induced by zymosan is limited to the site of injection. Furthermore, the injection of zymosan results in a stronger local reaction regarding the level of recruited immune cells, and injection of FITC-labelled zymosan enables the visualization of zymosan and thereby the core of the inflammation in the tissue. Therefore, zymosan was chosen to study the role of mast cells during the resolution phase of a local inflammation.

3.2. Role of mast cells during resolution of local inflammation

\subsubsection{Mast cells reside in anti-inflammatory region during resolution phase of zymosan-induced local inflammation}

To gain information on the internal structure of an inflammation induced by zymosan, especially in regard to the localization of mast cells and their cellular neighbourhood, MELC analysis was performed with paws from naïve mice as well as the during the peak (4 hours after zymosan-injection) and resolution (48 hours after zymosan-injection) of the inflammation. The automated MELC technology allows the sequential imaging with many antibodies on the same tissue sample, thereby providing neighbouring information on immune cells of distinct phenotypes in the tissue and their position in regard to zymosan. Visualization of zymosan as the core of the inflammation was enabled by injection of FITClabelled zymosan. Time points of the peak and the resolution of the inflammation were identified by mechanical hypersensitivity after injection of zymosan (10 $\mu \mathrm{l}, 12 \mathrm{mg} / \mathrm{ml})$ into the paw of mice. A significant increase of mechanical hypersensitivity was measured as early as 1 hour after injection of zymosan and kept increasing for at least 8 hours after the injection. Starting at 24 hours after injection, the mechanical hypersensitivity receded, returning to baseline after 5 days (Figure 12A). 
A

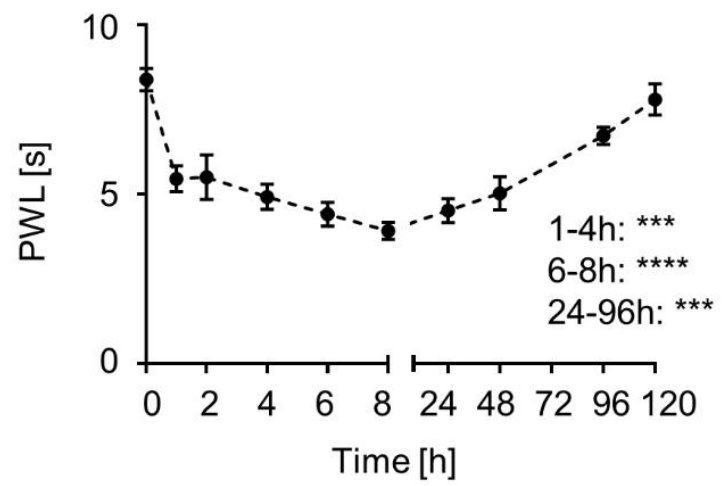

B

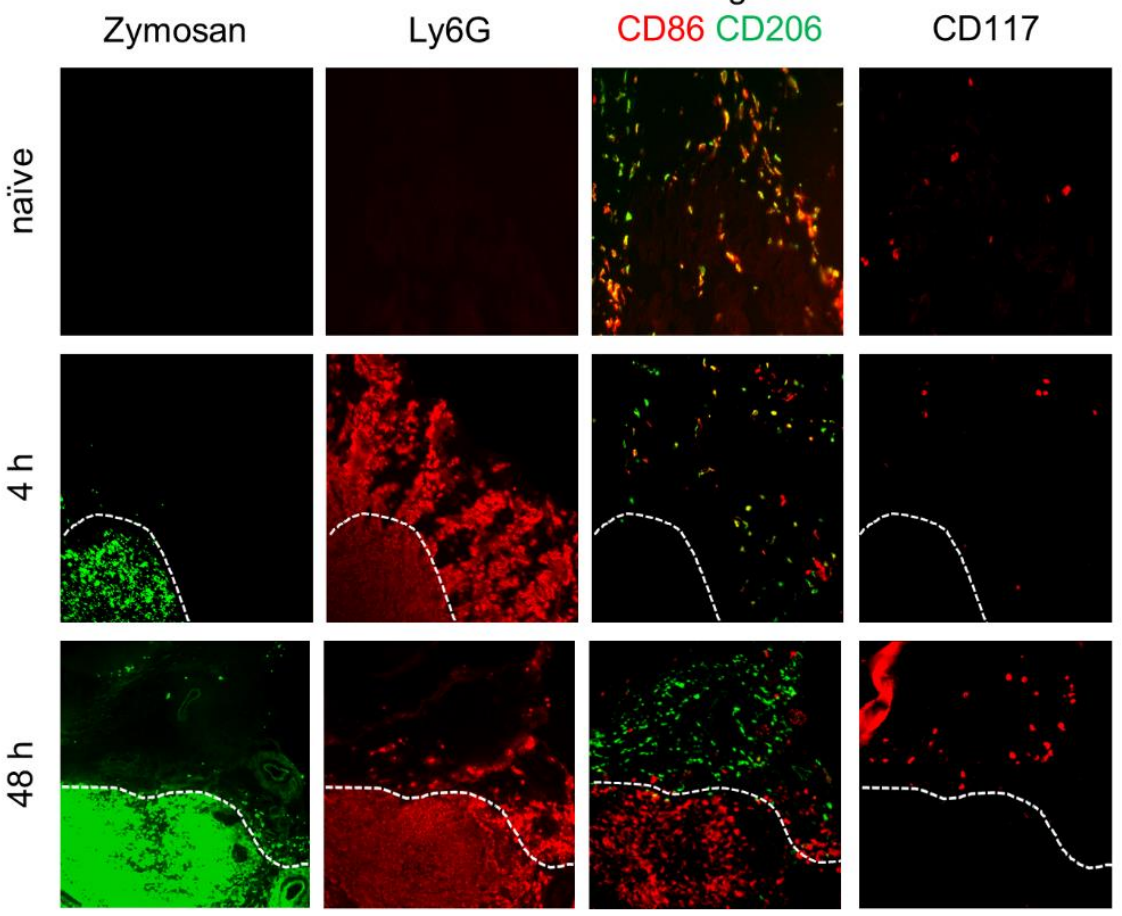

Figure 12: Mast cells are located in the anti-inflammatory region during the resolution phase, surrounding the zymosan core of the inflammation. Adapted from Kornstädt et al. (2021). (A) Mechanical paw withdrawal latencies in mice $(n=10)$ at indicated time points after injection of zymosan $(10 \mu l, 12 \mathrm{mg} / \mathrm{ml}$ in PBS). Data are mean \pm S.E.M., one-way ANOVA, Dunnett's test vs. baseline, ${ }^{* * *} p<0.001,{ }^{* * * *} p<0.0001$. (B) Representative images of immunohistological MELC staining of paws from naïve mice or $4 \mathrm{~h}$ or $48 \mathrm{~h}$ after injection of $12 \mathrm{mg} / \mathrm{ml}$ FITC-labelled zymosan. The dotted line indicates the outline of the zymosan-filled area in the tissue. Shown in false colors are FITC-labelled Zymosan (green), neutrophils (Ly6G, red), marker for pro-inflammatory cells (CD86, red), anti-inflammatory cells (CD206, green), and mast cells (CD117, red).

For the MELC analysis, neutrophils $\left(\mathrm{Ly}_{6 \mathrm{G}}{ }^{+}\right)$, mast cells $\left(\mathrm{CD} 117^{+}\right)$, CD86 and CD206 as proand anti-inflammatory markers respectively, were stained in the tissue with fluorescentlabelled antibodies (Figure 12B). In naïve paws, no neutrophils were detected. During the peak of the inflammation, neutrophils were located in and around the zymosan-filled area, fitting to their role as phagocytosing cells clearing the tissue from pathogens. Remarkably the staining for neutrophils appears to be more fine-grained within the zymosan-filled area, which 
could indicate that these cells have already undergone apoptosis, induced by a high uptake of zymosan by phagocytosis (Figure 12B, second row).

In naïve paws, resident macrophages ( $\mathrm{F} 4 / 80^{+}$Ly6C) can be found in the tissue, while at 4 hours post zymosan-injection infiltrating monocyte-derived macrophages (F4/80+ $\mathrm{Ly} 6 \mathrm{C}^{+}$) can be seen outside of the zymosan-filled area. At 4 hours, the macrophages are expressing both $\mathrm{CD} 6^{+}$and $\mathrm{CD}^{2} 26^{+}$at the same time, while at 48 hours two distinct macrophage populations can be identified, namely pro-inflammatory $\mathrm{CD} 6^{+}$and anti-inflammatory $\mathrm{CD}^{206}{ }^{+}$. Those populations reside in distinct regions of the tissue; while the pro-inflammatory macrophages are located in the zymosan-filled area, anti-inflammatory macrophages can be found surrounding the zymosan-filled area, but not within (Figure 12B, third row). Interestingly, at 48 hours post zymosan-injection mast cells can be found exclusively in the anti-inflammatory region next to the field of zymosan in the tissue (Figure 12B, last row).

To analyse the neighbourhood relations between the individual cell phenotypes in the tissue, quantitative analysis of the MELC images was performed using HistoCAT, a machine learning software (Schapiro et al., 2017; Schüffler et al., 2015). Single-cell segmentation was performed based on CD45 staining and propidiumiodide nuclei staining. Individual cell phenotypes were analysed by PhenoGraph, enabling the discrimination of different immune cells based on marker expression (Figure 13A,B). Within the cell clusters neutrophils were identified as $\mathrm{Ly}_{6 \mathrm{G}}+$, dendritic cells als $\mathrm{CD} 11 \mathrm{c}^{+} \mathrm{MHCll}^{+}$, eosinophils as $\mathrm{F} 4 / 80^{+}$Siglec $\mathrm{F}^{+}, \mathrm{M} 1$ macrophages as $\mathrm{CD}^{+} 6^{+}, \mathrm{M} 2$ macrophages as $\mathrm{CD}^{206}{ }^{+}$, and mast cells as CD117 ${ }^{+}$(Figure 13C). Phenograph analysis of resolution phase MELC images shows expression of antiinflammatory cytokines (IL-4, IL-13, IL-33) as well as the pro-inflammatory cytokine IL-1 $\beta$ in mast cells. Furthermore, PhenoGraph analysis of resolution phase MELC images show that neutrophils in the zymosan-filled area show a strong expression of CD11b, which fits to their presumed phagocytosing activity, while neutrophils without direct contact to zymosan show a less strong expression of CD11b (Figure 13C). Additionally, PhenoGraph analysis substantiates the observation that macrophages $\left(\mathrm{F} 4 / 80^{+}\right)$located in the zymosan-filled area are M1 (CD86 expression), while macrophages outside the zymosan-filled area are M2 (CD206 expression).

Neutrophils were absent in naïve paws and most prominent 4 hours after injection, starting to disappear again at 48 hours (Figure 12B, second row; Figure 14). Meanwhile macrophages were more Ly6G-positive 48 hours after injection compared to 4 hours after injection (Figure 14), reflecting the typical clearance of apoptotic neutrophils by macrophages during resolution of inflammation. 
A

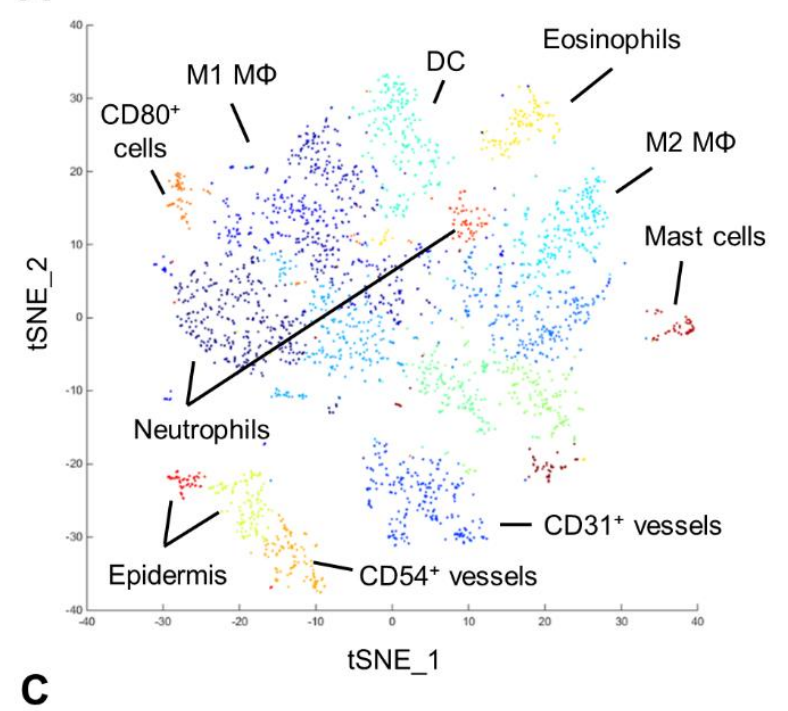

B Mast cells

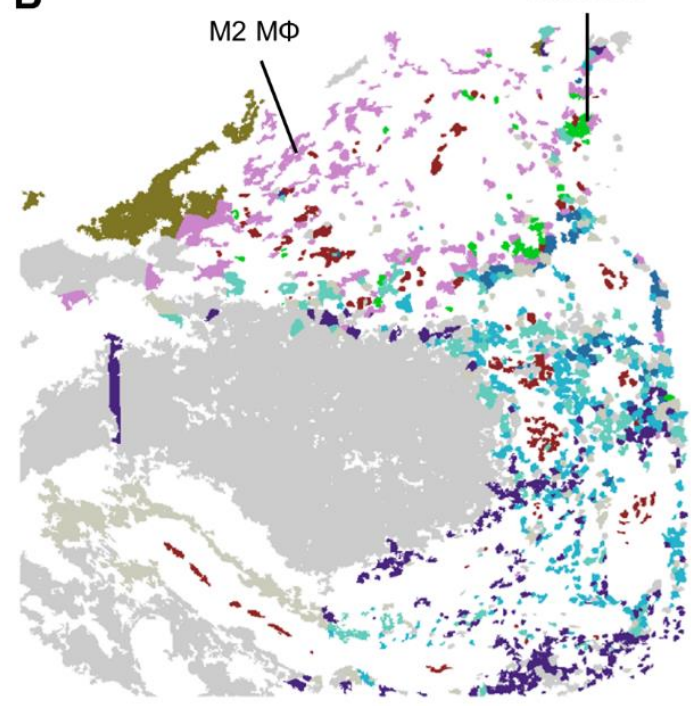

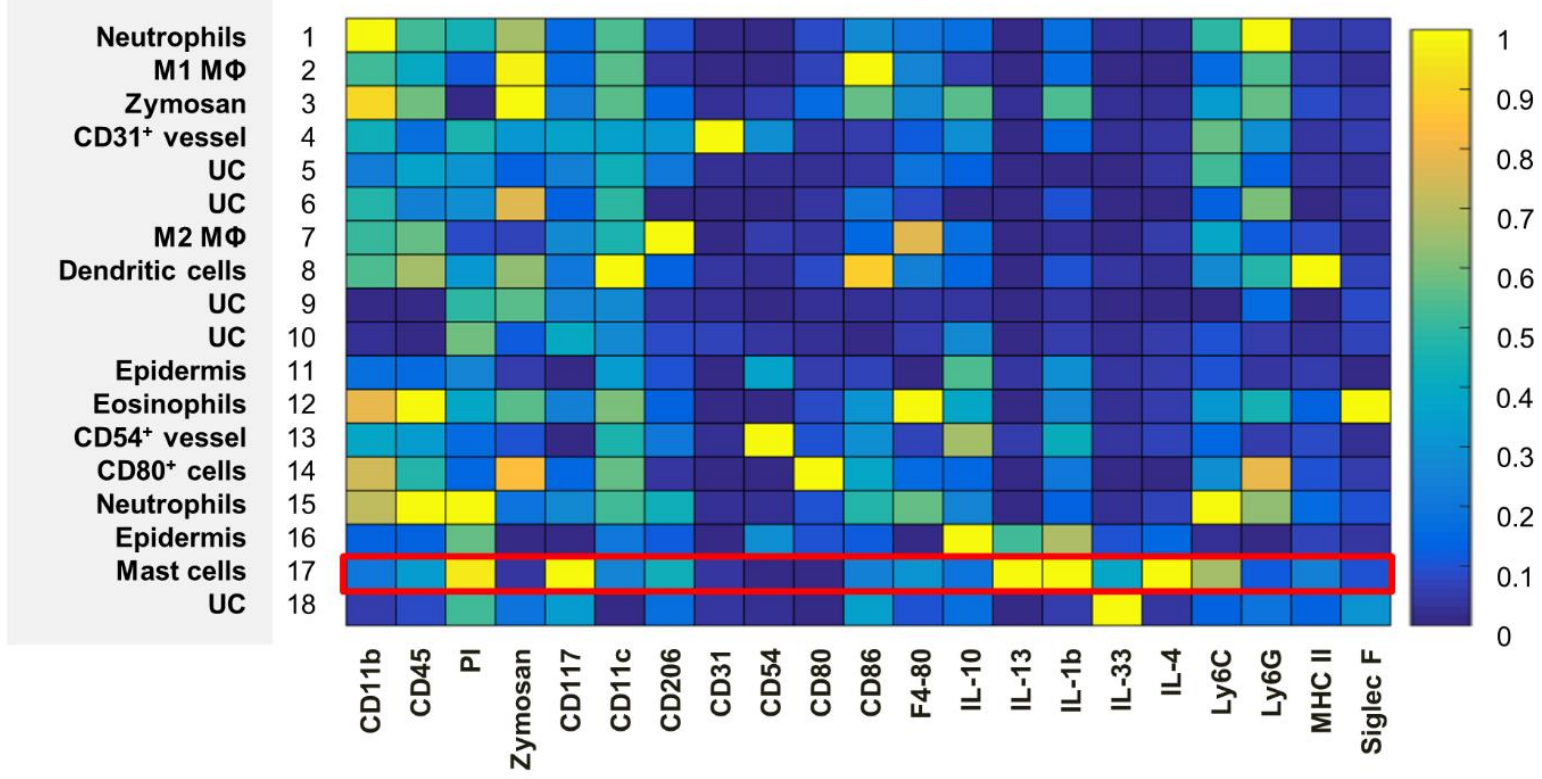

Figure 13: Identification and characterization of immune cell phenotypes in MELC images of inflamed

paws. Figure adapted from Kornstädt et al. (2021), analysis performed by Tim Schäufele. (A) Representative Barnes-Hut t-SNE (BH t-SNE) plot colored by cell clusters defined by PhenoGraph analysis. Underlying images originate from MELC analysis of mice paw $48 \mathrm{~h}$ after zymosan injection. The positions of some cell types are depicted in the plot. DC, dendritic cells; MФ, macrophages. (B) Representative image of single cells and their phenotype identified in the MELC image by cell segmentation depicted in their original location in the image of the paw. Underlying images originate from MELC analysis of mice paw $48 \mathrm{~h}$ after zymosan injection. Cells sharing the same phenotype are shown in the same color. The positions of mast cells and M2 macrophages (MФ) are depicted in the plot. (C) Representative PhenoGraph analysis of MELC images from mouse paw $48 \mathrm{~h}$ after zymosan-injection shows expression of anti-inflammatory cytokines (IL-4, IL-13, IL-33) and pro-inflammatory IL-1 $\beta$ in mast cells. Th heat map shows a PhenoGraph analysis of MELC data $(n=4)$ based on single-cell segmentation with CD45 and nuclei marker propidiumiodide (PI) using CellProfiler. Yellow depicts a strong expression and dark blue a low expression of markers, relative within each column. Marker expression of mast cells is highlighted by a red box. MФ, macrophages; UC, unidentified cluster. 
Analysis of the cellular microenvironment of mast cells during resolution of inflammation (48 hours post zymosan-injection) shows that they are most frequently neighbouring M2 macrophages and also frequently neighbouring eosinophils and dendritic cells (Figure 15A). M1 macrophages and neutrophils on the other hand are not included in the neighbourhood of mast cells (Figure 15A). The cellular neighbourhood of mast cells and M2 macrophages was not as pronounced in naïve mice or during the peak of the inflammation and increased significantly during the resolution of inflammation (Figure 15B).
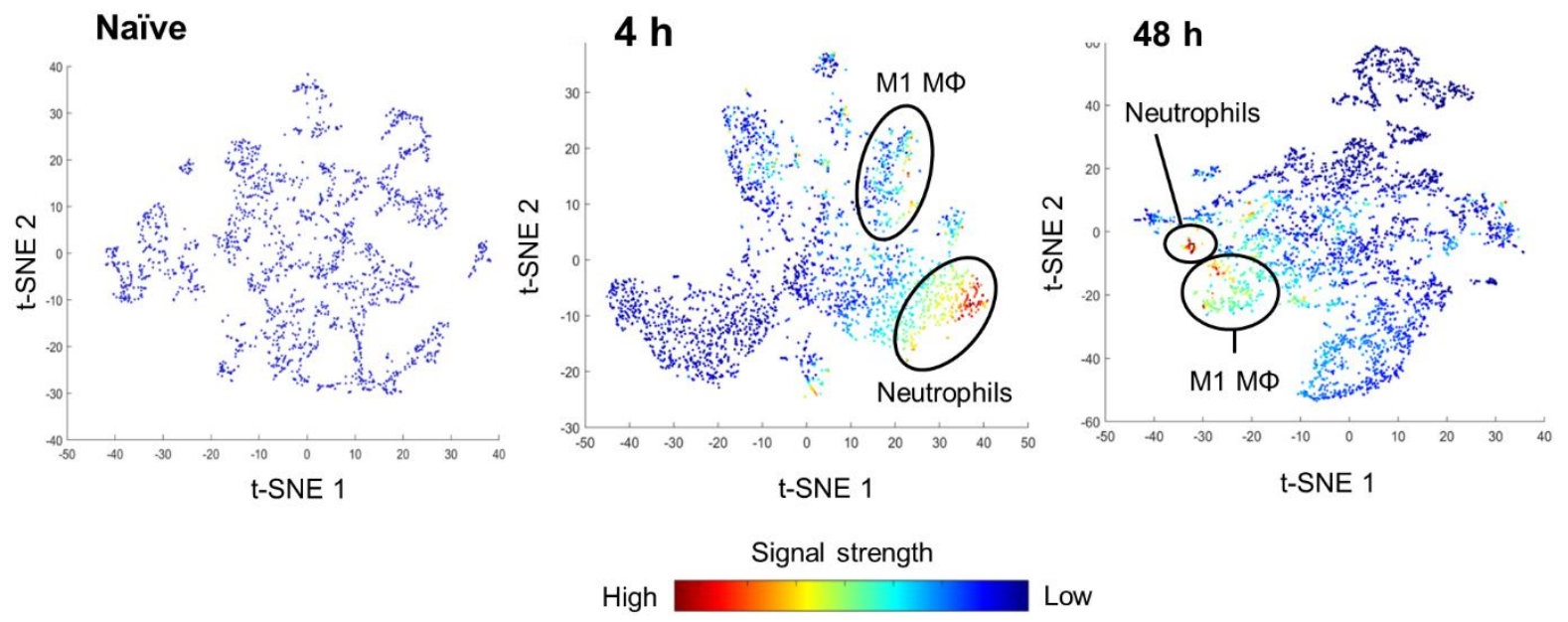

Figure 14: Efferocytosis increases during resolution of inflammation. Figure adapted from Kornstädt et al. (2021), analysis performed by Tim Schäufele. Representative BH t-SNE analysis of MELC images from mouse paw naïve, $4 \mathrm{~h}$ or $48 \mathrm{~h}$ after zymosan-injection showing time course of neutrophil occurrence based on Ly6G marker expression ( $n=3$, respectively). Neutrophil and M1 macrophage phenotype cluster are indicated by circles in t-SNE plots. No Ly6G is detected in naïve paws. Ly6G-positive macrophages at $4 \mathrm{~h}$ are less prominent as compared to $48 \mathrm{~h}$ reflecting the increased efferocytosis during resolution of inflammation. Single-cell segmentation is based on CD45 and nuclei marker using CellProfiler. M $\Phi$, macrophages.

Summarised MELC analysis of paws nicely displays the development and architecture of a local inflammation induced by zymosan. The infiltration of phagocytosing neutrophils to the zymosan-filled area, as well as the infiltration and polarisation of macrophages can be observed, and the division of the tissue in pro- and anti-inflammatory regions is revealed. Neighbourhood analysis furthermore shows that mast cells are located in the antiinflammatory region of the tissue in direct neighbourhood to M2 macrophages during the resolution of inflammation, and PhenoGraph analysis reveals the presence of antiinflammatory mediators in mast cells. All together pointing towards an anti-inflammatory or pro-resolving role of mast cells in this biological setting. 
A

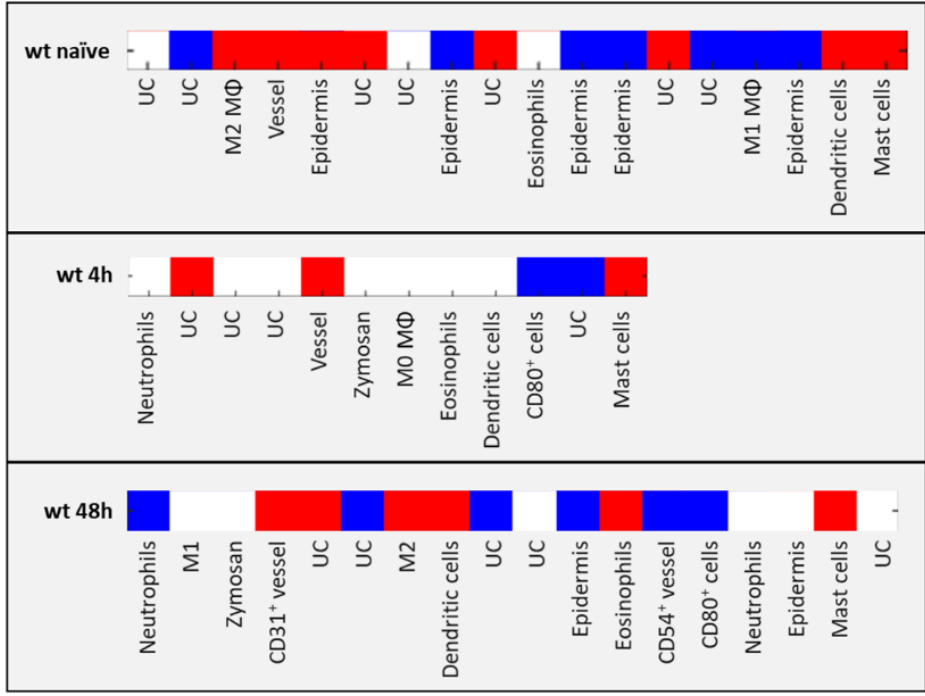

B

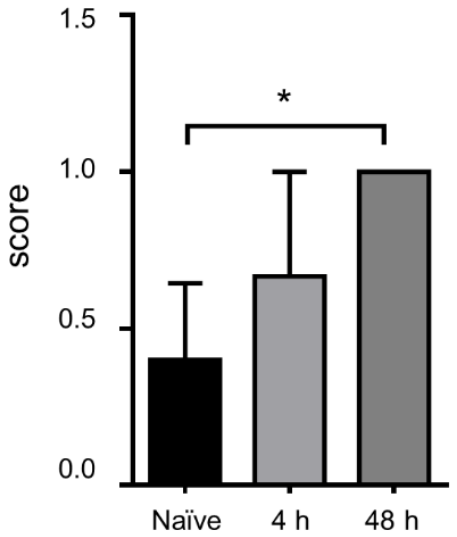

Figure 15: Mast cells are located in anti-inflammatory neighbourhood during resolution of zymosaninduced inflammation. Figure adapted from Kornstädt et al. (2021), analysis performed by Tim Schäufele. (A) Heat map showing a representative result of the neighbourhood analysis for mast cells in naïve paw and $4 \mathrm{~h}$ or $48 \mathrm{~h}$ after zymosan injection ( $\mathrm{n}=3-4)$. UC, unidentified cluster. Red depicts cells, which neighbour mast cells more frequently than they would in random permutations of cell cluster labels in each image set. Blue depicts cell clusters neighbour less frequently than with randomly permuted cell labels and white depicts cells cluster neighbours with random frequency. (B) Averaged score for mast cell neighbourhood regarding anti-inflammatory macrophages (M2 MФ) based on neighbourhood analysis of naïve paw or $4 \mathrm{~h}$ or $48 \mathrm{~h}$ after injection of zymosan $(n=3-4)$. A score was assigned for $r e d=1$, white $=0$, blue $=1$. Data are shown as mean \pm S.E.M., one-way ANOVA test, ${ }^{*} \mathrm{p}<0.05$.

\subsubsection{Mast cells influence macrophage phagocytosis and efferocytosis activity}

Since PhenoGraph analysis and neighbourhood analysis indicated for an anti-inflammatory, pro-resolving phenotype of mast cells, the effect of mast cells on the resolution of inflammation and on the immune cells involved was analysed in mast cell-deficient Mcpt5DTA Cre ${ }^{+}$mice and mast cell-sufficient Mcpt5-DTA Cre- mice. In these transgenic mice Cre is expressed under the mast cell specific promotor Mcpt5, leading to the excision of a loxP flanked stop cassette controlling the expression of diphtheria toxin alpha chain (DTA). The resulting mast cell specific expression of DTA leads to the ablation of mast cells in Mcpt5DTA Cre ${ }^{+}$mice, while maintaining an otherwise normal immune system. Accordingly, mast cell numbers in paws of Mcpt5-DTA Cre ${ }^{+}$mice were decreased around $80 \%$ compared to Mcpt5-DTA Cre mice (Figure 16A,C). The number of mast cells did not change significantly over the course of an inflammation in Mcpt5-DTA Cre mice (Figure 16A,B). Seeing that 
infiltrating immune cells during inflammation would distort the percentage of mast cells in the paw, mast cell numbers were normalized to resident macrophages (F4/80+/Ly6C-).
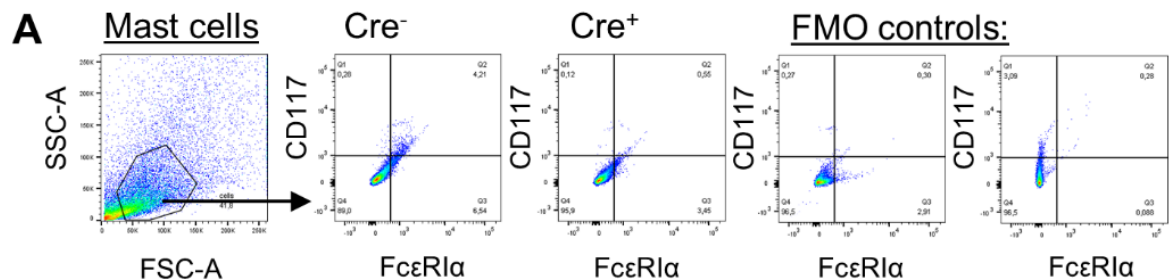

$\underline{\text { Resident macrophages }}$

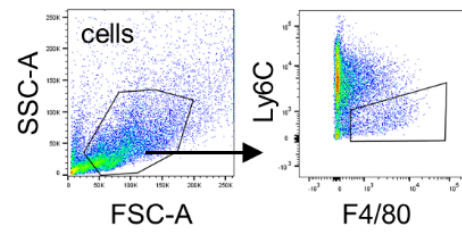

FMO controls:

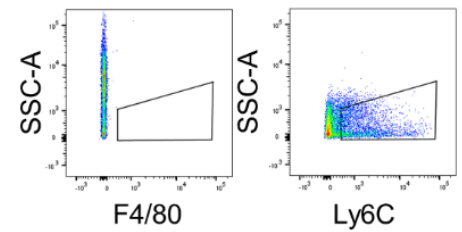

B

C
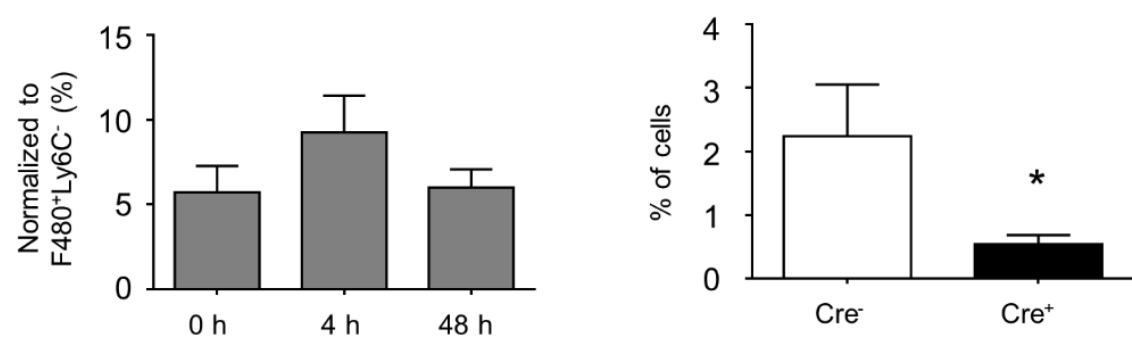

Figure 16: Mast cells are decreased in paws of Mcpt5-DTA Cre ${ }^{+}$mice compared to Cre- mice. Reprinted from Kornstädt et al. (2021). (A) Mast cells were identified as CD117+ FceRla ${ }^{+}$cells by flow cytometry. Resident macrophages were used for normalization and identified as F4/80+ Ly6C- cells. Exemplary flow plots of Mcpt5DTA Cre ${ }^{-}$and Cre $^{+}$samples as well as FMO controls for CD117, FceRla, F4/80 and Ly6C are shown. (B) Flow cytometry analysis of mast cells in paws of Mcpt5-DTA Cre- after injection of $10 \mu \mathrm{l} z y m o s a n(12 \mathrm{mg} / \mathrm{ml})$ at indicated time points $(n=4)$. Numbers of mast cells were normalized to resident macrophages. Data are shown as mean \pm S.E.M., one-way ANOVA, Dunnett's multiple comparison test. (C) Flow cytometry analysis of mast cells in paws of Mcpt5-DTA Cre ${ }^{-}$and Cre $^{+}$mice $(n=4)$. Data is shown as mean \pm S.E.M., unpaired t-test, one-tailed, ${ }^{*} p<0.05$.

Since the neighbourhood analysis showed that mast cells reside in the direct proximity of M2 macrophages during the resolution of inflammation, potential effects of mast cells on macrophages were analysed next by comparing the phenotype of macrophage populations 48 hours after injection of zymosan in Mcpt5-DTA $\mathrm{Cre}^{+}$and $\mathrm{Cre}^{-}$mice. Interestingly, PhenoGraph analysis of MELC images of inflamed paws showed a reduction of the neutrophil marker Ly6G in M1 macrophages (Figure 17, Figure 18). This indicates for a reduced efferocytosis of neutrophils by macrophages, an important process required for the successful resolution of inflammation. 

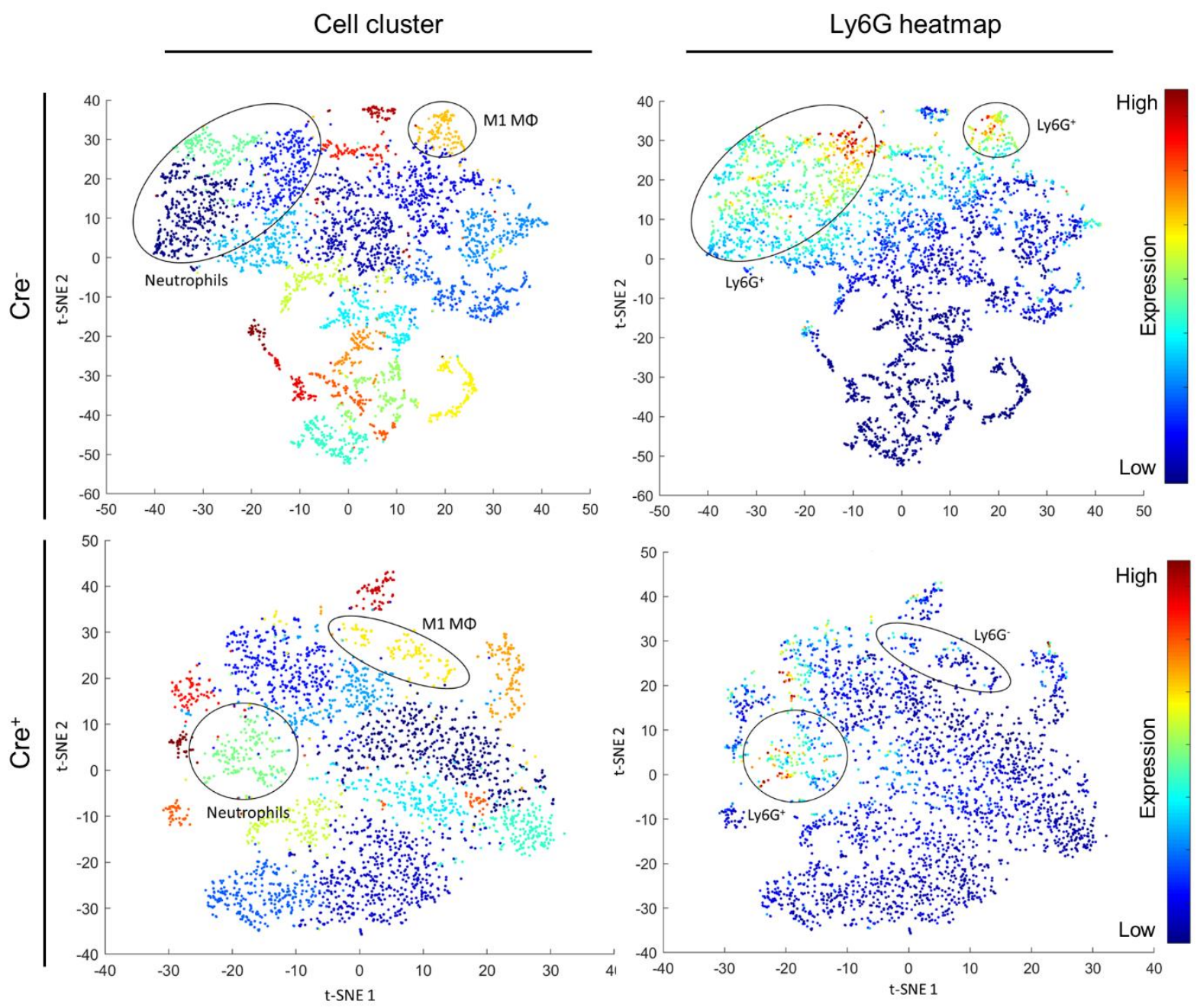

Figure 17: M1 macrophage phagocytosis of neutrophils is reduced in mast cell-deficient Mcpt5-DTA Cre ${ }^{+}$ mice compared to Cre- control mice. Figure adapted from Kornstädt et al. (2021), analysis performed by Tim Schäufele. Images show representative BH t-SNE analysis from Mcpt5-DTA Cre+ or Cre- mice $48 \mathrm{~h}$ after injection of zymosan. Plots on the left are colored by cell clusters defined by PhenoGraph analysis (Figure 18). Plots on the right are heatmaps for the neutrophil marker Ly6G. The position of cell phenotype clusters containing CD86 ${ }^{+}$

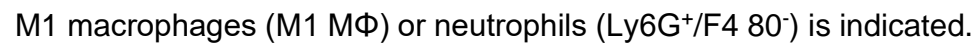

To analyse the reduced efferocytosis in Mcpt5-DTA $\mathrm{Cre}^{+}$mice seen in MELC analyses, polychromatic flow cytometry was performed. Therefore, Mcpt5-DTA Cre ${ }^{+}$and $\mathrm{Cre}^{-}$mice were injected with zymosan intraplantar and the inflamed tissue was analysed 48 hours after injection. Albeit mast cells have been shown to influence the recruitment of neutrophils during the onset of inflammation (De Filippo et al., 2013), no change in neutrophil numbers could be observed at this late time point (Figure 7A, Figure 19A). The numbers of macrophages, eosinophils and dendritic cells were also unaltered in mast cell-deficient mice (Figure 7A, Figure 19A). Since mast cells were located in the direct neighbourhood of M2 macrophages, the levels of $\mathrm{M} 1$ and $\mathrm{M} 2$ macrophages were also analysed, but the polarisation of macrophages regarding CD86 and CD206 expression was also not altered by the absence of mast cells 48 hours after injection of zymosan (Figure 7B, Figure 19B). 


\section{Mcpt5-DTA Cre-}

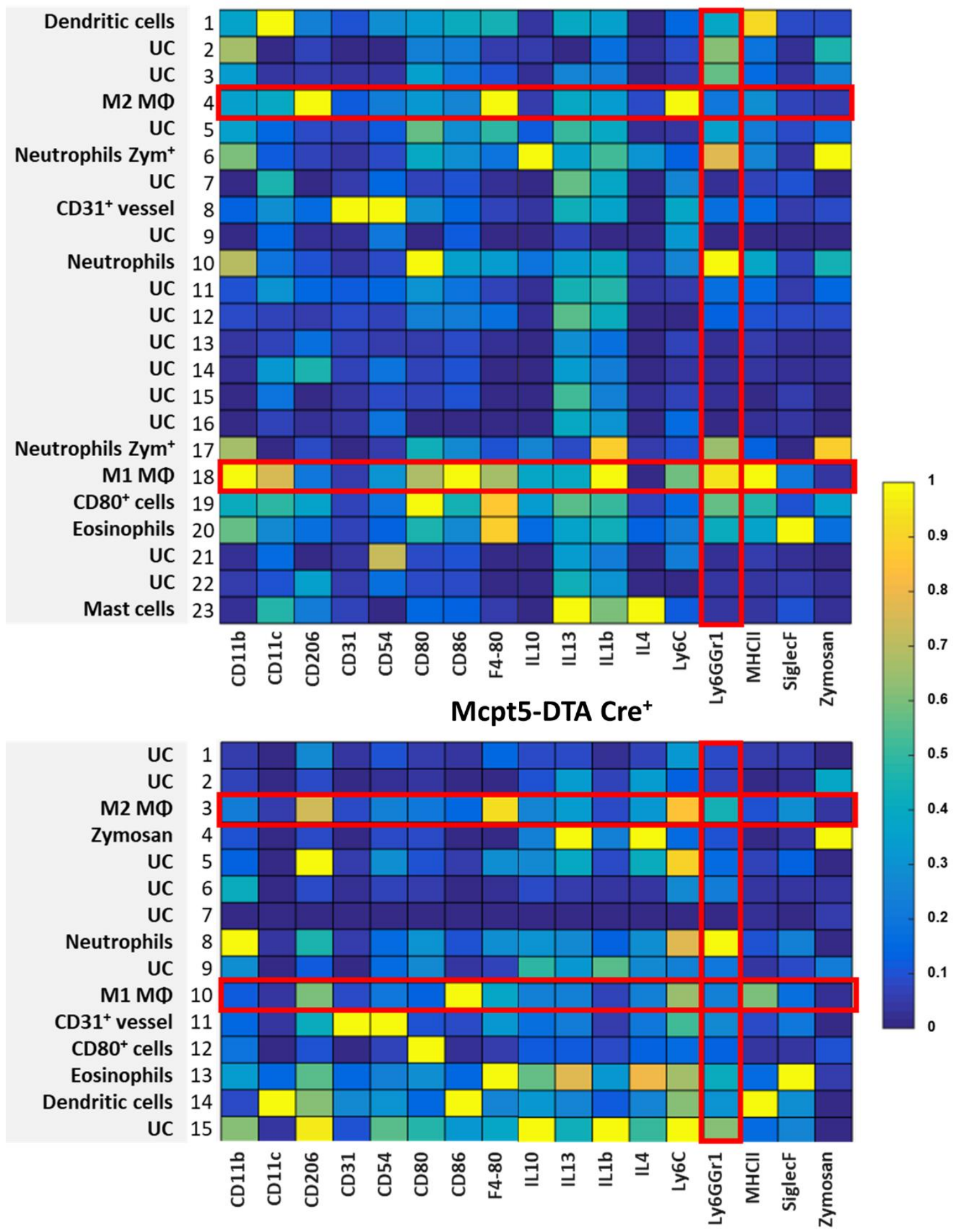

Figure 18: Decreased Ly6G signal in M1 macrophages in paws of mast cell-deficient Mcpt5-DTA Cre ${ }^{+}$ mice. Reprinted from Kornstädt et al. (2021), analysis performed by Tim Schäufele. Heat map of representative PhenoGraph analysis of MELC images from Mcpt5-DTA Cre ${ }^{+}$and Cre mouse paw $48 \mathrm{~h}$ after zymosan-injection ( $n=3$, respectively) based on single-cell segmentation with CD45 and nuclei marker using CellProfiler. Yellow depicts strong expression and dark blue low expression, all relative within one column. Marker expression in macrophage phenotype cluster (M1, M2) is marked by a horizontal red box. Expression of neutrophil marker Ly6G is marked by a vertical red box. MФ, macrophages; UC, unidentified cluster. 
Finally, since mast cells have been shown to increase the ability of macrophages to phagocytose neutrophils in mast cell-deficient C57BL/6 Kit ${ }^{\mathrm{W}-\mathrm{sh} / \mathrm{W}-\mathrm{sh}}$ mice, we analysed the phagocytic activity of macrophages and neutrophils by polychromatic flow cytometry. To determine the phagocytic activity, we injected $\mathrm{pH}$-sensitive pHrodo Red zymosan bioparticles (pHrodo-zymosan) into paws of Mcpt5-DTA Cre mice. When phagocytosed and inside acidic lysosomes, the $\mathrm{pH}$-sensitive pHrodo-zymosan particles turn brightly fluorescent, enabling the detection by flow cytometry. Neither the percentage of phagocytosing neutrophils ( $\mathrm{F} 4 / 80^{-}$ Ly6G $^{+}$pHrodo-zymosan ${ }^{+}$), nor the mean fluorescence intensity (MFI) of pHrodo-zymosan in neutrophils was altered in mast cell deficient Mcpt5-DTA Cre ${ }^{+}$mice compared to Cre- mice 48 hours after injection of pHrodo-zymosan (Figure 19C,D). Remarkably, both the percentage of phagocytosing macrophages $\left(\mathrm{F} 4 / 80^{+}\right.$pHrodo-zymosan ${ }^{+}$) and the amount of zymosan phagocytosed by them (MFI of pHrodo-zymosan in $\mathrm{F} 4 / 80^{+}$) was significantly reduced in mast cell-deficient Mcpt5-DTA Cre+ mice (Figure 19C,E). Not only the uptake of zymosan by macrophages was decreased in the absence of mast cells, but also the uptake of neutrophils, as determined by the percentage of intracellular Ly6G in macrophages (Figure $19 F, G)$.

In summary, we could confirm the effect of mast cells on the phagocytosing and efferocytosing activity of macrophages observed in C57BL/6 Kit ${ }^{W-s h} / \mathrm{W}$-sh mice using Mcpt5DTA Cre mice. 

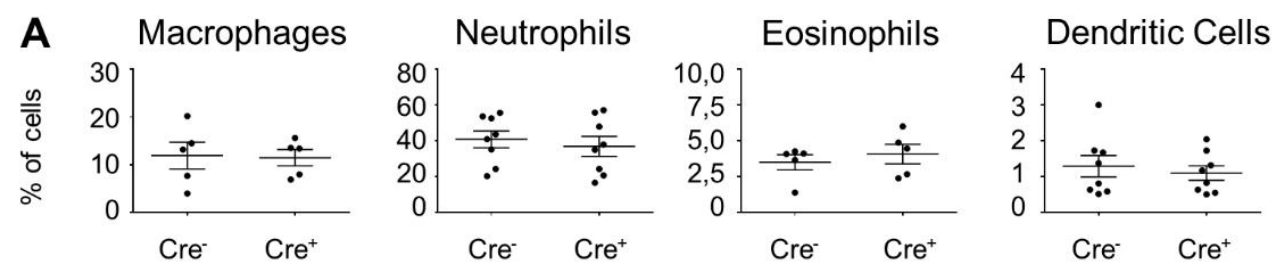

\section{B M1 Macrophages M2 Macrophages}
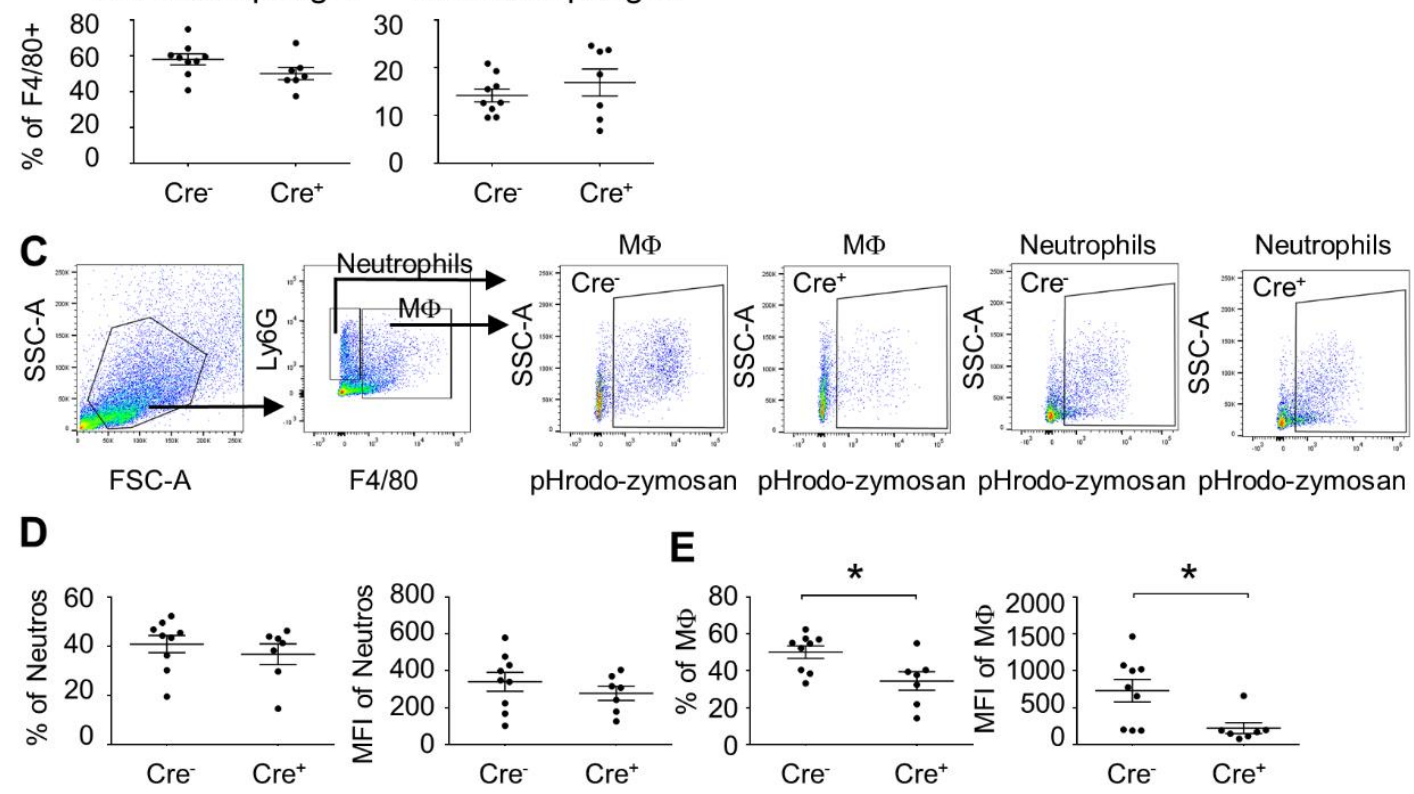

$\mathbf{F}$

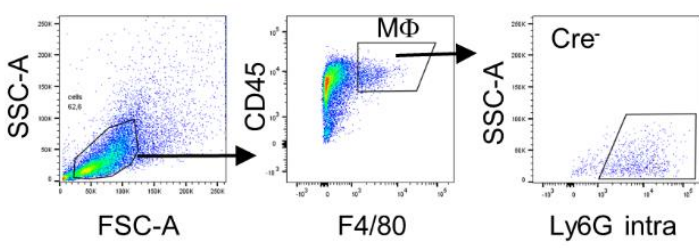

G

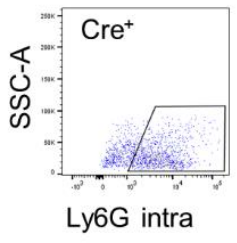

G Neutrophils

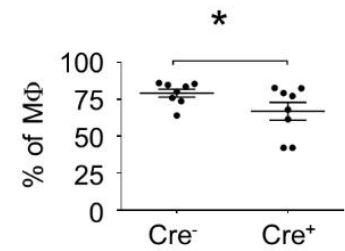

Figure 19: Mast cells influence phagocytosing activity of macrophages during resolution phase of zymosan-induced inflammation. Reprinted from Kornstädt et al. (2021). (A) Number of neutrophils, macrophages, eosinophils and dendritic cells in Mcpt5-DTA Cre- and $\mathrm{Cre}^{+}$mice $48 \mathrm{~h}$ after injection of zymosan (10 $\mu \mathrm{l}, 12 \mathrm{mg} / \mathrm{ml}$ in PBS). Data are shown in \% of all cells as mean \pm S.E.M. $(\mathrm{n}=5-9)$, unpaired two-tailed t-test. (B) Number of M1-like (CD86 $)$ macrophages and M2-like $\left(\mathrm{CD}^{+} 06^{+}\right)$macrophages $48 \mathrm{~h}$ after injection of zymosan

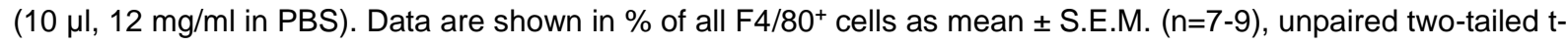
test. (C) Gating strategy for flow cytometry analysis of phagocytosis of pHrodo Red zymosan by macrophages $\left(\mathrm{F} 4 / 80^{+}\right)$and neutrophils $\left(\mathrm{F} 4 / 80^{-} / \mathrm{Ly} 6 \mathrm{G}^{+}\right)$. (D,E) Phagocytosis of pHrodo Red zymosan $(10 \mu \mathrm{l}, 12 \mathrm{mg} / \mathrm{ml}$ in PBS) by neutrophils (panel D) or macrophages (panel E) in Mcpt5-DTA Cre- and $\mathrm{Cre}^{+}$mice $48 \mathrm{~h}$ after zymosan injection. Data are mean \pm S.E.M. $(n=7-9)$, unpaired two-tailed t-test, ${ }^{*} p<0.05,{ }^{* *} p<0.01$. (F) Gating strategy for flow cytometry analysis of phagocytosis of neutrophils by macrophages. Intracellular staining of $\mathrm{Ly} 6 \mathrm{G}^{+}$neutrophils was performed. (G) Decreased phagocytosis of neutrophils (intracellular Ly6G) by macrophages (F4/80+). Data are mean \pm S.E.M. $(n=7-9)$, unpaired two-tailed t-test, ${ }^{*} p<0.05$. 
3.2.3. Screening of cytokines involved in inflammatory response after zymosaninjection in mast cell-deficient mice compared to control mice

In order to identify mediators released by mast cells that may influence phagocytic, efferocytic activity of neighbouring cells and the course of the inflammation, the levels of 23 cytokines, chemokines and growth factors were analysed in paws of mast cell-deficient Mcpt5-DTA $\mathrm{Cre}^{+}$and in mast cell-sufficient Mcpt5-DTA Cre mice. The levels of these mediators were determined by a multiplex cytokine assay, allowing for the analysis of all 23 mediators at once in the same tissue sample. Ipsi- and contralateral paws of Mcpt5-DTA $\mathrm{Cre}^{+}$and Cre- mice were analysed 48 hours after injection of zymosan into the ipsilateral paw.

Comparing the inflamed tissue of the ipsilateral paws with the control tissue of the contralateral paws within one genotype, the levels of 9 mediators were not significantly altered 48 hours after the treatment at all, namely IL-3, IL-10, IL-12 (p40), IL-12 (p70), IL-13, IL-17, Eotaxin, IFN-y, and TNFa. The pro-inflammatory IL-1 $\beta$, as well as the chemoattractants G-CSF, CCL2, CCL3, CCL4, and CCL5 were increased in the zymosaninjected paws, regardless of the genotype, while the pleiotropic cytokine IL-2 was decreased in inflamed paws from both genotypes (Figure 20). Meanwhile, the pro-inflammatory IL-1 $\alpha$ was only decreased in ipsilateral paws of Mcpt5-DTA $\mathrm{Cre}^{+}$mice, although no significant differences can be observed between $\mathrm{Cre}^{-}$and $\mathrm{Cre}^{+}$tissue. IL-5, another pleiotropic cytokine, was only increased in ipsilateral paws of Mcpt5-DTA Cre+ mice, but not in ipsilateral paws of Cre mice. IL-6, IL-9, and GM-CSF on the other hand were only increased in Cre ipsilateral paws. However, there is no significant difference for these mediators between $\mathrm{Cre}^{-}$and $\mathrm{Cre}^{+}$ mice.

IL-4 and CXCL1 on the other hand, which are both increased in ipsilateral paws compared to contralateral paws of the same genotype, are significantly decreased in mast cell-deficient mice compared to control mice within the inflamed tissue (Figure 20). IL-4, the first cytokine described to be released by mast cells (M. A. Brown et al., 1987; McLeod et al., 2015), is known to promote the alternative activation of macrophages towards an M2 phenotype and suppress the production of pro-inflammatory cytokines (Goerdt et al., 1999; S. Gordon, 2003; Hart et al., 1989). CXCL1 is known to be involved in the recruitment of neutrophils by mast cells (De Filippo et al., 2013) and in wound healing (Castela et al., 2017). 

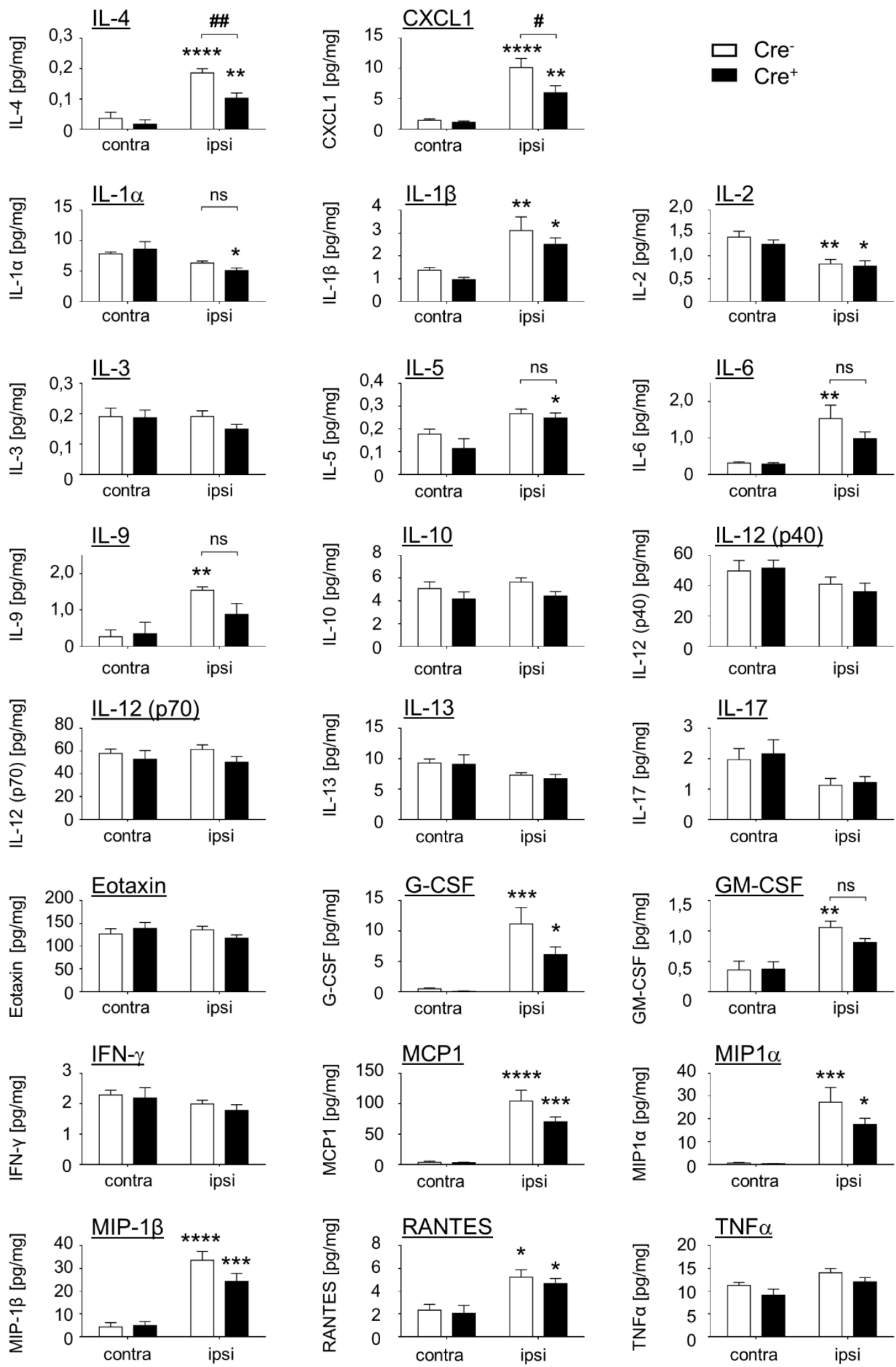

Figure 20: IL-4 and CXCL1 are decreased in mast cell-deficient mice during inflammation. Reprinted from Kornstädt et al. (2021). Levels of cytokines, chemokines and growth factors in contra- and ipsilateral paws $48 \mathrm{~h}$ after injection of zymosan (12 mg/ml) in Mcpt5-DTA Cre- and Cre ${ }^{+}$mice. Data are mean \pm S.E.M. ( $\left.n=6\right)$. Two-way ANOVA, Tukey's multiple comparison test, significance between ipsi- and contralateral paws is presented by ${ }^{*} p<0.05,{ }^{* *} p<0.01,{ }^{* *} p<0.001,{ }^{* * *} p<0.0001$, significance between genotypes is presented by $\#<0.05, \# \# p<0.01$. 
However, 48 hours after zymosan injection neither an increase of M1 macrophages, nor a decrease of neutrophil numbers was observed in mast cell-deficient mice (Figure 7A,B; Figure 19A,B). If there is no effect on early neutrophil recruitment by mast cells in this model or the effect can not be observed anymore at this late time point needs to be further elucidated. However, the decrease in efferocytosis observed in flow cytometry analysis can't be explained by the known effects of IL-4 and CXCL1 on the phenotype of macrophages or the recruitment of neutrophils, respectively, since neither of these effects is observable at this time point.

Besides cytokines and chemokines, lipid mediators play a key role in the inflammatory response. Prostanoids are a subclass of lipid mediators that are significantly increased in inflamed tissue and have pro- as well as anti-inflammatory properties (Ricciotti \& Fitzgerald, 2011). Bioactive prostanoids generated in vivo are the four prostaglandins $P G E_{2}, P G D_{2}$, $\mathrm{PGI}_{2}$, and $\mathrm{PGF}_{2 \mathrm{a}}$, as well as $\mathrm{TXA}_{2}$. To further characterize the influence of mast cells on a local inflammation we measured the levels of these mediators or their more stable derivates in the case of $P \mathrm{Pl}_{2}$ and $\mathrm{TXA}_{2}$, namely 6-keto $P G F_{1 \alpha}$ and $\mathrm{TXB}_{2}$, respectively, in ipsi- and contralateral paws of Mcpt5-DTA Cre- and $\mathrm{Cre}^{+}$mice.

There is no significant difference in the level of $\mathrm{PGE}_{2}$ and 6-keto $\mathrm{PGF}_{1 \alpha}$ between genotypes or ipsi- and contralateral paws observable (Figure 21). $\mathrm{PGF}_{2 \alpha}$ is significantly reduced in the inflamed paws both in Mcpt5-DTA Cre ${ }^{+}$and Cre- mice. $\mathrm{PGD}_{2}$ meanwhile is only significantly reduced in the ipsilateral paws of Mcpt5-DTA $\mathrm{Cre}^{+}$mice compared to their contralateral paws, while $\mathrm{TXB}_{2}$ is increased in ipsilateral paws of Mcpt5-DTA Cre+ mice compared to their contralateral paws. However, no significant difference between Mcpt5-DTA Cre- and $\mathrm{Cre}^{+}$ mice can be detected for any of the mediators.
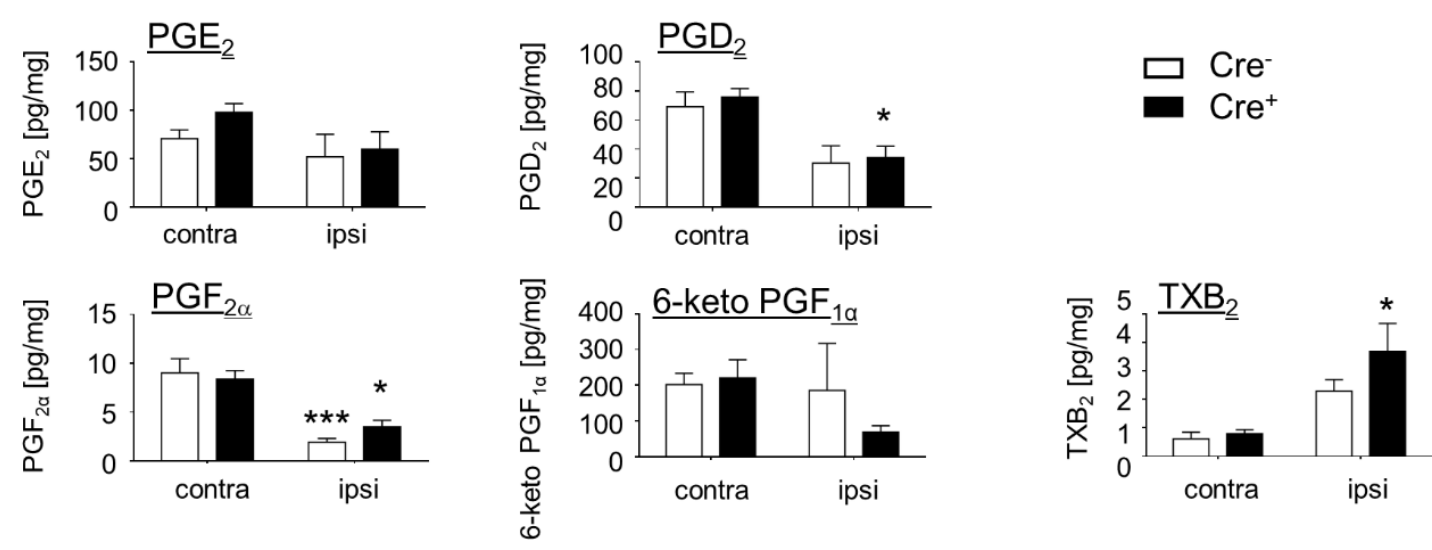

Figure 21: Levels of prostanoids during local inflammation in Mcpt5-DTA Cre mice. Levels of $P E_{2}, P D_{2}$, $\mathrm{PGF}_{2 \alpha}$, 6-keto PGF $1 \alpha$ and $\mathrm{TXB}_{2}$ in contra- and ipsilateral paws in Mcpt5-DTA Cre- and Cre ${ }^{+}$mice $48 \mathrm{~h}$ after injection of zymosan $(10 \mu \mathrm{l}, 12 \mathrm{mg} / \mathrm{ml})$. Data are mean \pm S.E.M. $(\mathrm{n}=4)$. Two-way ANOVA, Tukey's multiple comparison test, significance between ipsi- and contralateral paws is presented by ${ }^{*} p<0.05,{ }^{* *} p<0.001$. 


\subsection{Identification of mediators produced by mast cells}

Since mast cells are localized to relatively small defined areas of the paw tissue, mediators released by them might be increased locally, but might be difficult to detect in the context of the whole tissue. To enable a more sensitive detection of mast cell-derived mediators, the supernatant of bone marrow-derived mast cells (BMMCs) was analysed after induction with various stimuli.

\subsubsection{Analysis of purity and maturity of bone marrow-derived mast cells}

To verify the purity and maturity of BMMCs, they were analysed by flow cytometry after differentiating the cells for 4 weeks in mast cell medium. Therefore, the cells were stained with anti-mouse CD117/KIT-PE and anti-mouse FC\&RI $\alpha$-FITC.

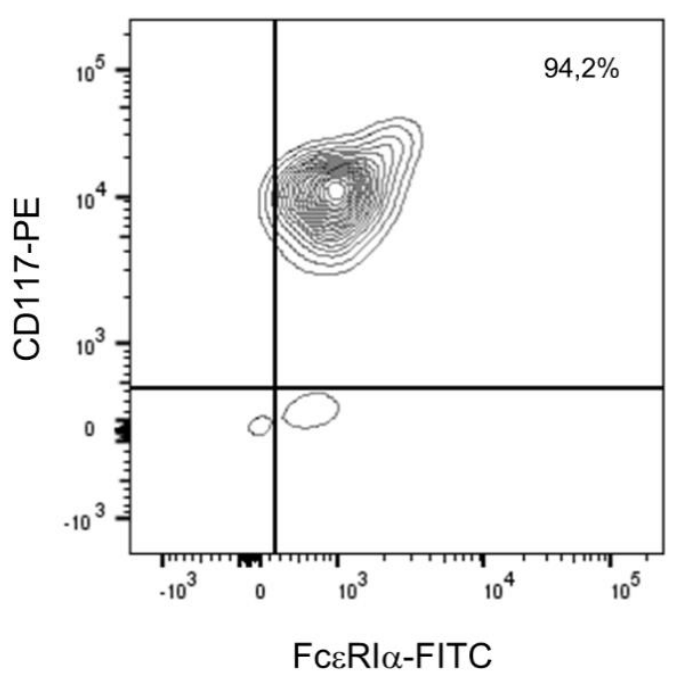

Figure 22: Flow cytometry analysis shows high purity of BMMCs matured according to standard protocol. Reprinted from Kornstädt et al. (2021). BMMCs were stained with antibodies against CD117 and FcERI $\alpha$ after 4 weeks of cultivation and identified as double positive cells.

The cell surface marker CD117, also known as mast/stem cell growth factor (SCF) or protooncogene c-Kit is widely used as a mast cell marker, but is also expressed by a variety of hematopoietic progenitors, as well as basophils, myeloid dendritic cells, melanocytes and others (Broudy, 1997). The high-affinity IgE receptor FceRla plays a major role in allergic responses by mediating the degranulation of mast cells, but is also found on eosinophils, basophils and epidermal Langerhans cells (Stone et al., 2010). The double-staining of CD117 and Fc\&Rl $\alpha$ is unique for mast cells and is commonly used to verify their identity. The 
flow cytometry analysis showed that the cultivation of bone marrow-derived mast cell precursors yielded cells expressing the typical mast cell surface markers (Figure 22).

\subsubsection{Newly synthesized mediators released by BMMCs after TLR induction}

The pro-inflammatory IL-1 $\beta$ and the anti-inflammatory IL-10 are mediators known to be released by mast cells in response to TLR signaling (Sandig \& Bulfone-Paus, 2012b). The anti-inflammatory effects of mast cell-derived IL-10 has been shown in various studies (Chan et al., 2013; Depinay et al., 2006; Grimbaldeston et al., 2007). To determine the optimal time point to detect mediators released by mast cells after TLR activation, the concentration of IL10 and IL-1 $\beta$ in the supernatant was measured by ELISA at different time points after induction of BMMCs with zymosan $(10 \mu \mathrm{g} / \mathrm{ml})$ and LPS (100 $\mathrm{ng} / \mathrm{ml})$.
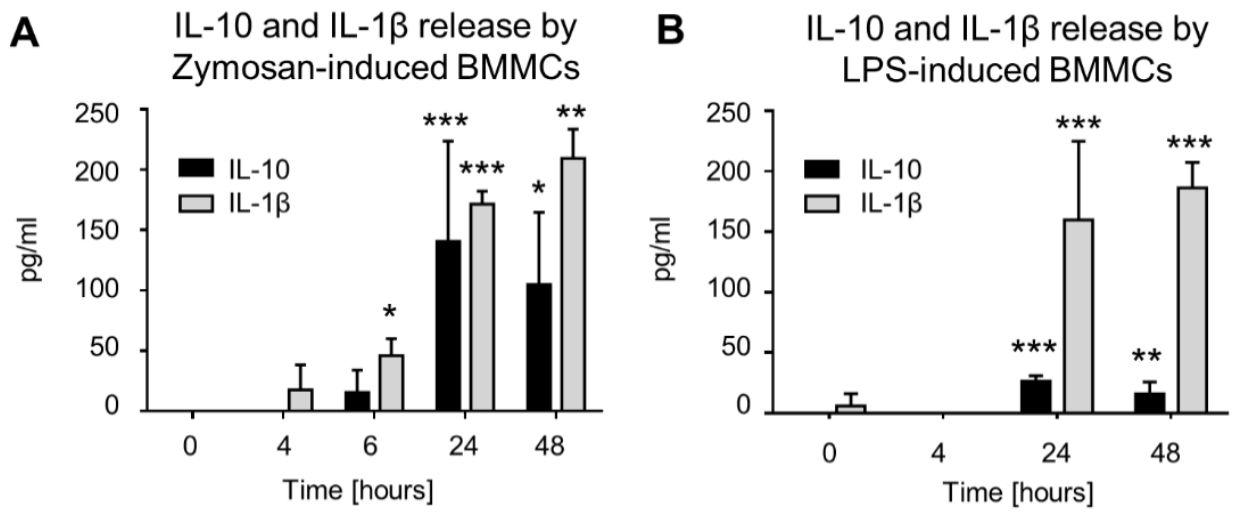

Figure 23: Timecourse of release of IL-10 and IL-1 $\beta$ by mast cells after induction with zymosan or LPS. Figure adapted from Kornstädt et al. (2021). (A) Concentration of IL-10 and IL-1 $\beta$ in the supernatant of BMMCs induced with zymosan $(10 \mu \mathrm{g} / \mathrm{ml})$ at the indicated time points. (B) Concentration of IL-10 and IL-1 $\beta$ in the supernatant of BMMCs induced with LPS $(100 \mathrm{ng} / \mathrm{ml})$ at the indicated time points. Data are shown as mean \pm S.E.M. $(n=4)$. One-way ANOVA, Dunnett's multiple comparison test for IL-10 and IL-1 $\beta$, compared to $0 \mathrm{~h}$ control, ${ }^{*} \mathrm{p}<0.05,{ }^{* *} \mathrm{p}<0.01,{ }^{* * *} \mathrm{p}<0.001,{ }^{* * * *} \mathrm{p}<0.0001$.

Although no significant difference was detected for the level of IL-10 and IL-1 $\beta$ between mast cell-deficient and -sufficient mice in vivo, both mediators were released by mast cells in response to zymosan and to LPS (Figure 20, Figure 23A,B). Since mast cells are relatively rare cells in the tissue, changed mediator levels might only be detected locally, but not in the context of the whole paw tissue. A significant increase of IL-1 $\beta$, but not IL-10, was already detected 6 hours after zymosan induction, while 4 hours after zymosan or LPS there was yet no significant increase of IL-1 $1 \beta$. The highest concentration of IL-1 $\beta$ in the supernatant was detected after 24 hours and 48 hours both in zymosan and LPS stimulated cells. Both for zymosan and LPS induced cells, the highest concentration of the anti-inflammatory IL-10 
was detected after 24 hours of incubation; therefore 24 hours was chosen for detection of other cytokines. While the concentration of IL-1 $\beta$ was in a similar range after induction with zymosan and LPS, the release of IL-10 was markedly higher after zymosan-induction than after LPS-induction.

Both CXCL1 and IL-4, which were reduced in the inflamed tissue of mast cell-deficient mice compared to inflamed tissue of mast cell-sufficient mice, were induced by zymosan and by LPS (Figure 20; Figure 24A). IL-13, a cytokine with high similarities to IL-4, is also released by mast cells in response to both zymosan and LPS, despite no differences due to treatment or genotype could be detected for the level of IL-13 in vivo (Figure 20; Figure 24A). Meanwhile TGF- $\beta$, another cytokine associated with TLR activation (He et al., 2016), was neither induced by zymosan nor LPS in BMMCs (Figure 24A).
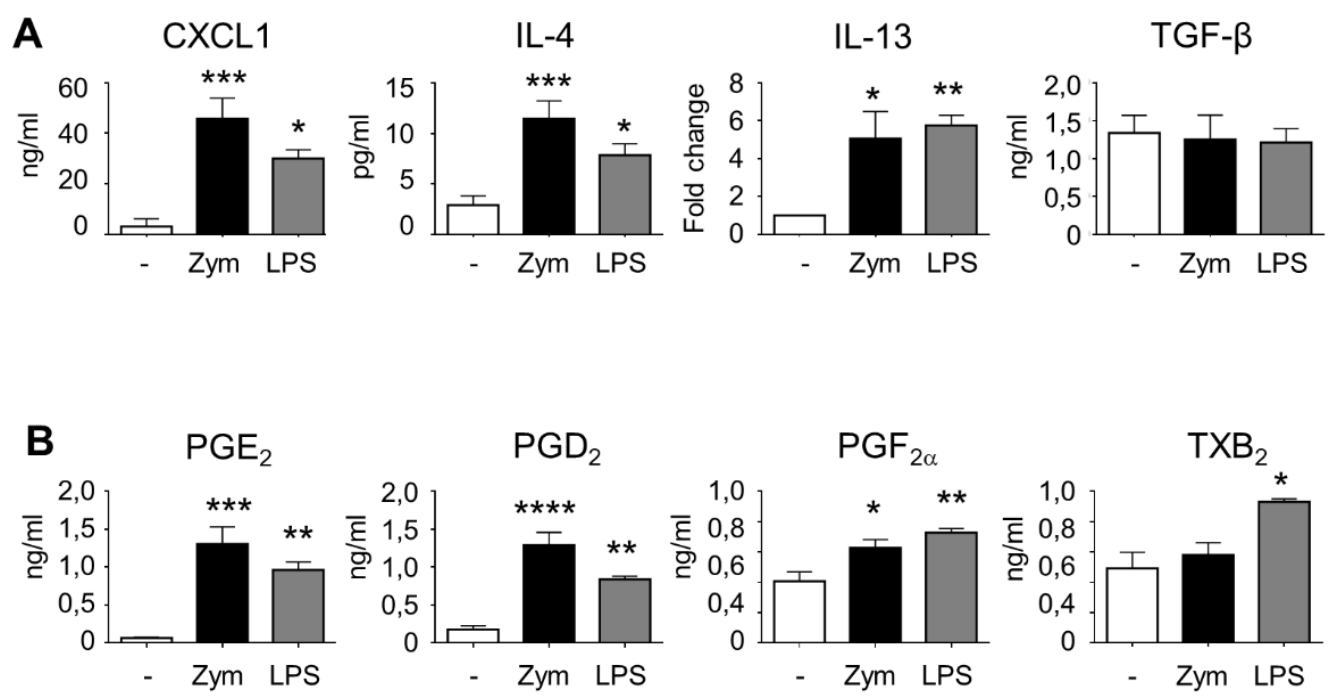

Figure 24: Chemokines and prostanoids released by mast cells after induction with zymosan or LPS. (A) Concentration of CXCL1 $(n=4), I L-4(n=7), I L-13(n=4)$ and TGF- $\beta(n=5)$ in the supernatant of BMMCs $24 \mathrm{~h}$ after induction with zymosan $(10 \mu \mathrm{g} / \mathrm{ml})$ or LPS $(100 \mathrm{ng} / \mathrm{ml})$. (B) PGE2, PGD, $\mathrm{PGF}_{2 \alpha}$ and $\mathrm{TXB}_{2}(\mathrm{n}=5)$ in the supernatant of BMMCs $24 \mathrm{~h}$ after induction with zymosan $(10 \mu \mathrm{g} / \mathrm{ml})$ or LPS $(100 \mathrm{ng} / \mathrm{ml})$. Data are presented as mean \pm S.E.M. One-way ANOVA, Dunnett's multiple comparison test compared to control, ${ }^{*} p<0.05,{ }^{* *} p<0.01$, ${ }^{* * *} \mathrm{p}<0.001$.

The prostanoids $\mathrm{PGE}_{2}, \mathrm{PGD}_{2}$ and $\mathrm{PGF}_{2 \alpha}$, which are known to play a role in inflammatory processes, were also released by BMMCs 24 hours after induction with zymosan and LPS (Figure 24B). Another known mast cell product, $\mathrm{TXB}_{2}$, was only released by BMMCs after induction with LPS, not zymosan (Figure 24B), while concentrations of 6-keto PGF Fa $_{1 \alpha}$ were all below the lower limit of quantification (data not shown here).

In conclusion, several mast cell-derived mediators with anti-inflammatory and pro-resolving properties could be identified after induction with the TLR ligands zymosan and LPS. Among 
these, IL-1 $\beta, I L-4$, and $\mathrm{PGE}_{2}$ have been demonstrated to increase phagocytosis in macrophages (Daseke et al., 2020; Schenk et al., 2014; Wainszelbaum et al., 2006).

\subsubsection{Induction of BMMCs with non-TLR signals}

Since mast cells are surrounded by a variety of other cells in the tissue during inflammation, mediators derived from neighbouring cells might also induce a response in mast cells. The immunoregulatory cytokine IL-13 is known to promote mast cell-mediated anaphylaxis and is predominantly produced by CD4 T cells, but also by basophils, eosinophils, mast cells and others (McLeod et al., 2015). IL-13 is structurally and functionally related to IL-4 and binds to a receptor complex consisting of the IL-13 binding proteins IL-13R $\alpha 1$ and IL-13R $\alpha 2$ and the receptor subunit IL-4R $\alpha$ (McCormick \& Heller, 2015). Sphingosine-1-phosphate (S1P) is a lipid mediator released by dying cells to trigger phagocytotic uptake and has been shown to influence mast cells in allergic diseases (Kugelberg, 2016; Olivera \& Rivera, 2011; Saluja et al., 2017). S1P signals through a family of $G$ protein coupled S1P receptors, two of them being expressed by mast cells. Lysophosphatidylcholines (LysoPCs) are a group of lipids that are increased during inflammation and oxidative stress and have various functions (Aiyar et al., 2007; Law et al., 2019; Qin et al., 2014). To analyse if the release of the cytokines IL10 and IL-1 $\beta$ is induced by these signals, mast cells were treated with these substances for 24 hours and then the supernatant was analysed by ELISA. As can be seen in Figure 25, neither of these signals induced the release of IL-1 $\beta$ or IL-10 in BMMCs.

A typical trait of mast cells is the rapid and massive release of mediators by degranulation after $\operatorname{lgE}$ receptor-mediated activation. To analyse if mast cells release the anti-inflammatory cytokine IL-10 in response to the activation of the $\operatorname{lgE}$ receptor, the cells were sensitized with DNP-specific mouse monoclonal IgE antibodies and then stimulated with DNP-BSA. The binding of DNP to the $\operatorname{lgE}$ antibodies leads to a clustering of FceRl $\alpha$, which causes the activation and degranulation of the mast cell. As a control for the induction of degranulation, the release of histamine was analysed. Histamine is one of the pre-formed mediators stored in mast cell granules and released within seconds after activation (Moon et al., 2014). Histamine was released by mast cells in response to IgE-mediated activation as expected (Figure 26A), showing that the BMMCs used herein are able to undergo this classic morphologic release. Release of IL-10 was analysed 24 hours after induction again, where the highest concetration was detected before. However, IgE-mediated activation did not induce the release of IL-10 in mast cells (Figure 26A). 

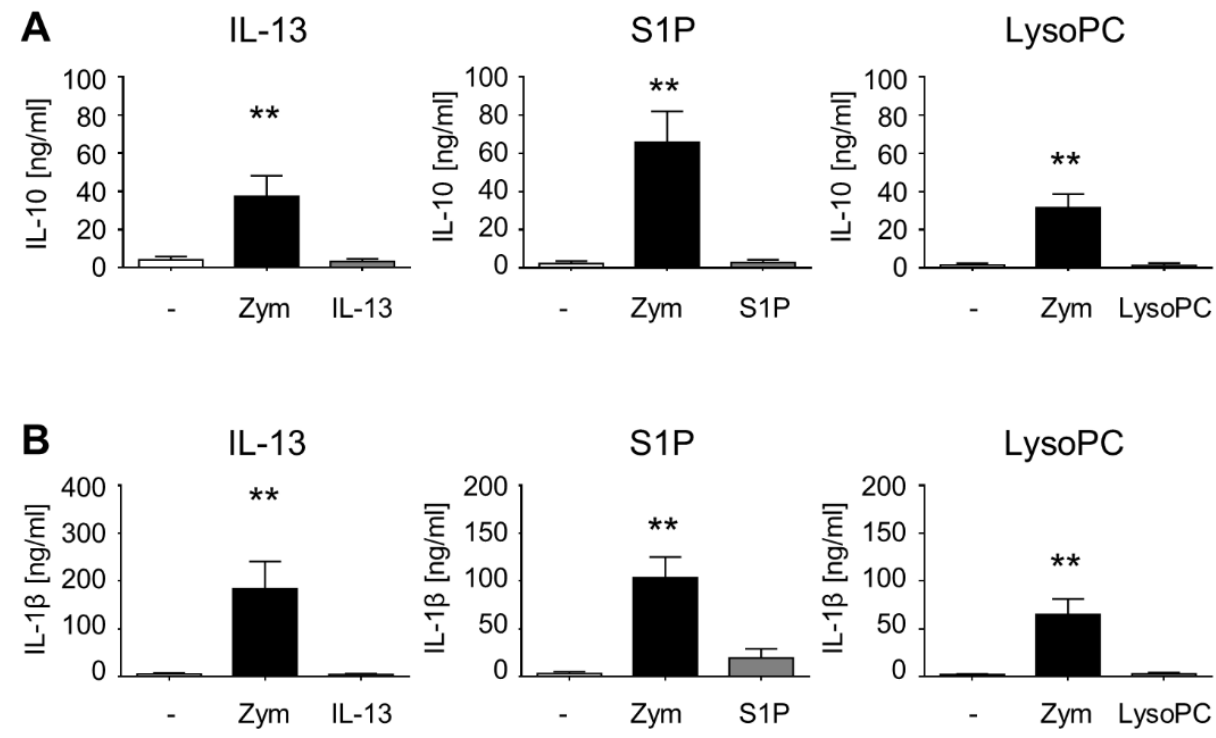

Figure 25: No release of IL-10 and IL-1 $\beta$ in response to IL-13, S1P or LysoPC. (A) Concentration of IL-10 in supernatant of BMMCs after induction with zymosan (Zym), $30 \mathrm{ng}$ IL-13, $5 \mu \mathrm{M} \mathrm{S1P}$, or $100 \mathrm{ng} 7 \mathrm{ml}$ LysoPC. (B) Concentration of IL-1 $\beta$ in supernatant of BMMCs after induction with zymosan (Zym), $30 \mathrm{ng}$ IL-13, $5 \mu \mathrm{M}$ S1P, and $100 \mathrm{ng} / \mathrm{ml}$ LysoPC. Data are presented as mean \pm S.E.M. $(\mathrm{n}=3-4)$. One-way ANOVA compared to unstimulated control (-), Dunnett's multiple comparison test, ${ }^{* *} \mathrm{p}<0.01$.

To further investigate the pathway involved in the release of the anti-inflammatory cytokine IL-10 by mast cells, the enzyme COX was inhibited by Diclofenac. The lipid mediator $\mathrm{PGE}_{2}$, which is generated by COX, has been demonstrated to enhance the production of IL-10 in previous studies (Alvarez et al., 2009; Harizi \& Gualde, 2006). Incubation of BMMCs before zymosan-induction completely inhibited the production of $\mathrm{PGE}_{2}$, demonstrating the effectiveness of the COX-inhibitor Diclofenac (Figure 26B). The decrease of IL-10 after treatment with Diclofenac (Figure 26B) is in accordance with previous studies demonstrating the role of prostanoid synthesis in the regulation of IL-10 production after induction with zymosan.

In conclusion, we investigated the effect of several mediators typically present during a local inflammation on mast cells and identified the TLR agonists LPS and zymosan as potent inducers of cytokine production. Other mediators potentially present in the neighbourhood of mast cells during resolution of inflammation, such as IL-13, S1P, and LysoPC on the other hand did not induce cytokine release in mast cells. Furthermore, we could rule out lgEmediated degranulation as a source for IL-10 release and we could show that COX-mediated prostanoid synthesis is involved in the production of IL-10 after TLR-mediated activation of mast cells. 

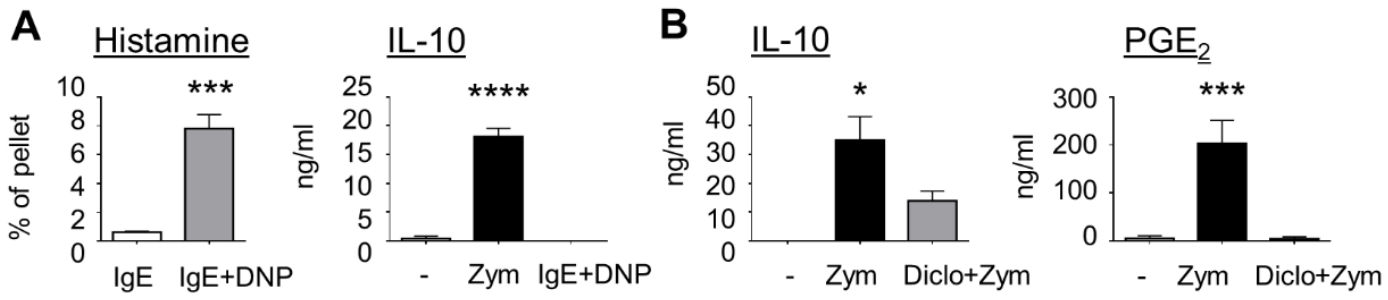

Figure 26: Release of IL-10 does not depend on IgE-mediated degranulation but on COX signaling. (A) Concentration of histamine (5 min after induction) or IL-10 (24 h after induction) in supernatant of unstimulated BMMCs (-) or after incubation with $1 \mu \mathrm{g} / \mathrm{ml}$ DNP-specific IgE only (lgE), induction with $100 \mathrm{ng} / \mathrm{ml}$ DNP-BSA conjugate after $\mathrm{IgE}$ incubation (IgE+DNP), or induction with $10 \mu \mathrm{g} / \mathrm{ml}$ zymosan $(Z y m)$. Data are presented as mean \pm S.E.M. $(n=4-5)$. Unpaired t-test, two tailed for histamine and one-way ANOVA compared to unstimulated control (-), Dunnett's multiple comparison test for IL-10, ${ }^{* * *} \mathrm{p}<0.001,{ }^{* * * *} \mathrm{p}<0.0001$. (B) Concentration of IL-10 or PGE2 in supernatant of unstimulatd BMMCs (-), BMMCs $24 \mathrm{~h}$ after induction with $10 \mu \mathrm{g} / \mathrm{ml}$ zymosan (Zym), or BMMCs induced for $24 \mathrm{~h}$ with $10 \mu \mathrm{g} / \mathrm{ml}$ zymosan after pre-treatment with $30 \mu \mathrm{g} / \mathrm{ml}$ Diclofenac (Diclo+Zym) for $2 \mathrm{~h}$. Data are presented as mean \pm S.E.M. $(n=4-5)$. One-way ANOVA compared to unstimulated control, Dunnett's multiple comparison test, ${ }^{*} \mathrm{p}<0.05,{ }^{* * *} \mathrm{p}<0.001$.

Besides typical anti-inflammatory cytokines like IL-10, further mediators with the potential to enhance phagocytosis in macrophages like IL-1ß, IL-4, IL-13, and PGE 2 (Berry et al., 2007; Daseke et al., 2020; Korns et al., 2011; Schenk et al., 2014; Szanto et al., 2010; Wainszelbaum et al., 2006; Welch et al., 2003) were identified after induction of BMMCs with TLR agonists zymosan and LPS. Since the targeted approach utilized here narrows the possibility to detect mast cell-derived mediators, mRNA sequencing was performed in a next step as an untargeted approach to further elucidate the response evoked in zymosaninduced BMMCs.

\subsubsection{RNA-sequencing as untargeted approach to identify mediators produced by zymosan-stimulated mast cells reveals type I IFN response}

For an untargeted screening of the response evoked in mast cells by stimulation with zymosan, RNA sequencing was performed. The mRNA of unstimulated cells ( 0 hours), and cells stimulated for 24 hours and 48 hours with $10 \mu \mathrm{g} / \mathrm{ml}$ zymosan was analysed and compared to identify transcriptional changes induced by TLR2 activation. Unstimulated cells served as the control condition, while 24 hours and 48 hours of treatment were chosen since the highest concentration and an already decreasing concentration of IL-10 were detected at these time points after zymosan-induction (Figure 23A), indicating for a strong reaction and the beginning resolution phase of the inflammatory response. 
3.3.4.1. Quality assessment of samples, libraries and sequencing data

To ensure that only the RNA of mature mast cells was used for sequencing and that cells of other phenotypes possibly present in the BMMC culture were excluded, the cells were sorted by FACS before RNA isolation. Fully differentiated mast cells were identified by staining positive for both CD117 and FceRla, after exlusion of debris and doublettes (Figure 8A-E). Around 1 million cells per sample were obtained by sorting. The supernatant of the cells used for sequencing was assayed by ELISA for the release of IL-10. All cultures used for sequencing released IL-10 after zymosan induction, demonstrating that a response to the stimulus was successfully evoked (Figure 27).

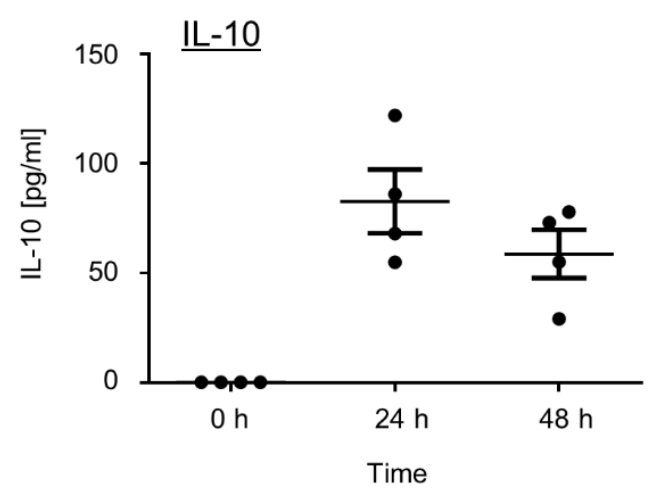

Figure 27: IL-10 release by BMMCs used for RNA sequencing. IL-10 in supernatant of BMMCs induced with $10 \mu \mathrm{g} / \mathrm{ml}$ zymosan was analysed at the indicated time points to verify successful stimulation of the cells.

RNA extraction from FACS sorted cells using the RNeasy Micro Kit (Qiagen) resulted in approximately 100 to $300 \mathrm{ng}$ RNA in $14 \mu \mathrm{l}$ RNase-free water per sample. The RNA integrity number (RIN) of these samples ranges between 6,0 and 8,3, (Table 1) as determined on an Agilent 2100 Bioanalyzer using an RNA 6000 Pico Chip (Agilent). The RIN classifies the integrity of RNA in values from 1 to 10, with 1 for the most degraded RNA and 10 for perfectly intact RNA. The obtained quality and quantity of RNA meets the input criteria for QuantSeq 3'mRNA FWD (Lexogen) library preparation, which requires at least $500 \mathrm{pg}$, better 10 to $500 \mathrm{ng}$ RNA in $5 \mu$ land would accept RNA with a lower RIN.

Illumina-compatible libraries were generated with QuantSeq 3'mRNA-Seq Kit Library Prep Kit (Lexogen). Concentration and size of the libraries assessed by Qubit ds DNA HS assay (Thermo Fisher) and a High Sensitivity DNA Chip (Agilent) was used to calculate the required input of each library to generate an equimolar mix for sequencing. Furthermore, the High Sensitivity DNA Chip (Agilent) was used to assess the quality and purity of the samples, since contaminants like adapter dimers or primer dimers can affect clustering efficacy and estimation of library concentrations by Qubit (Thermo Fisher). Precise estimation of the 
concentration of the libraries is crucial to avoid over- or underclustering of the flow cell, which can lead to low data quality and output. The analysis showed that the libraries were in the expected size range, free of contaminants and with no sign of overcycling. All libraries were pooled and used for sequencing on a NextSeq500 (Illumina).

Tabel 1: Quantity and quality of samples used for mRNA sequencing.

$\begin{array}{lllll}\text { Sample } & \text { Average } \\ \text { SNA concentration RIN DNA library Qubit } & \text { library size }\end{array}$

\begin{tabular}{ccccc}
\hline $1-0 \mathrm{~h}$ & $12.905 \mathrm{pg} / \mu \mathrm{l}$ & 8,2 & $1,270 \mathrm{ng} / \mu \mathrm{l}$ & $317 \mathrm{bp}$ \\
\hline $2-0 \mathrm{~h}$ & $13.563 \mathrm{pg} / \mu \mathrm{l}$ & 6,9 & $0,990 \mathrm{ng} / \mu \mathrm{l}$ & $296 \mathrm{bp}$ \\
\hline $3-0 \mathrm{~h}$ & $9.211 \mathrm{pg} / \mu \mathrm{l}$ & 7,3 & $2,100 \mathrm{ng} / \mu \mathrm{l}$ & $359 \mathrm{bp}$ \\
\hline $4-0 \mathrm{~h}$ & $23.133 \mathrm{pg} / \mu \mathrm{l}$ & 6,8 & $2,900 \mathrm{ng} / \mu \mathrm{l}$ & $334 \mathrm{bp}$ \\
\hline $1-24 \mathrm{~h}$ & $7.311 \mathrm{pg} / \mu \mathrm{l}$ & 6,8 & $1,130 \mathrm{ng} / \mu \mathrm{l}$ & $276 \mathrm{bp}$ \\
\hline $2-24 \mathrm{~h}$ & $16.747 \mathrm{pg} / \mu \mathrm{l}$ & 7,5 & $0,792 \mathrm{ng} / \mu \mathrm{l}$ & $315 \mathrm{bp}$ \\
\hline $3-24 \mathrm{~h}$ & $10.499 \mathrm{pg} / \mu \mathrm{l}$ & 7,6 & $1,290 \mathrm{ng} / \mu \mathrm{l}$ & $317 \mathrm{bp}$ \\
\hline $4-24 \mathrm{~h}$ & $15.331 \mathrm{pg} / \mu \mathrm{l}$ & 7,0 & $2,000 \mathrm{ng} / \mu \mathrm{l}$ & $327 \mathrm{bp}$ \\
\hline $1-48 \mathrm{~h}$ & $13.236 \mathrm{pg} / \mu \mathrm{l}$ & 8,3 & $0,906 \mathrm{ng} / \mu \mathrm{l}$ & $295 \mathrm{bp}$ \\
\hline $2-48 \mathrm{~h}$ & $13.511 \mathrm{pg} / \mu \mathrm{l}$ & 7,6 & $0,940 \mathrm{ng} / \mu \mathrm{l}$ & $328 \mathrm{bp}$ \\
\hline $3-48 \mathrm{~h}$ & $14.614 \mathrm{pg} / \mu \mathrm{l}$ & 6,0 & $7,40 \mathrm{ng} / \mu \mathrm{l}$ & $339 \mathrm{bp}$
\end{tabular}

Data quality metrics in the BaseSpace Sequence Hub (Illumina) showed that the sequencing run was successful; each of the 12 samples was distributed across 4 lanes of the flow cell, with each sample represented more or less equally with $6,3 \%$ to $10,8 \%$ reads identified per sample. In total, $42,24 \mathrm{Gbp}$ of high-quality data was yielded, with $83,56 \%$ bases reaching $Q \geq 30$, meaning that base call accuracy is $99,9 \%$. Cluster density was optimal, with 268 $271 \mathrm{~K} / \mathrm{mm}^{2}$ and more than 500 reads passing filter. In summary, the sequencing yielded maximal output while maintaining very high data quality. 


\subsubsection{Differential expression analysis of RNA sequencing data shows}

differences between untreated and zymosan-treated samples

Subsequent to sequencing, data analysis was performed to gain information on the differentially expressed genes between untreated BMMCs (0 hours) and BMMCs treated for 24 hours and 48 hours with zymosan. After preprocessing the data to remove adapter sequences and low-quality data, the reads were mapped against the mouse reference genome. On average $76 \%$ of reads could be uniquely mapped to genes. $16 \%$ of reads were overlapping with more than one gene (ambigious), 2\% were mapped to too many loci and $5 \%$ were not mapped because they were too short. The percentage of mapped reads shows that the overall sequencing accuracy is good and there is no contaminating DNA.

Following read mapping, the gene expression is quantified to enable the comparison of gene expression levels between the samples. Since only one fragment per transcript is generated by using the QuantSeq 3'mRNA-Seq Kit Library Prep Kit (Lexogen) correction for gene length is not necessary and raw read counts are sufficient to obtain accurate gene expression values.

Next, a principal component analysis (PCA) was performed to reveal the similarities and differences between the samples. When comparing sequencing data, the number of genes can be regarded as dimensions of a sample. To be able to identify correlations (or lack there of) between the 12 samples regarding a large number of differentially expressed genes, it is necessary to reveal patterns underlying this high-dimensional set of data. PCA is a method to reduce the number of dimensions of a complex dataset, while the essence of the data is captured in a few principal components which convey the most variation in the dataset. Each dot in the PCA plot represents the transcription profile of one of the samples. Samples that are highly correlated cluster together, while samples with strong differences in the transcription profile are distant from each other on the principal component axis. The first principal component axis (PC1) reveals the most variation, while the second principal component axis (PC2) reveals the second most variation. Therefore, differences among clusters along the PC1 axis are larger than the similar-looking distances along the PC2 axis.

There are two main clusters of samples identifiably in the PCA plot, 0 hours and 24 hours/48 hours (Figure 28A). This shows that the main difference lies between time point 0 hours and 24 hours/48 hours, which indicates that the main differences between the samples are due to the treatment. The samples of the 24 hours time point are more distant from the 0 hours samples than the 48 hours samples are, showing that the variation between 24 hours and 0 hours is slightly bigger than between 48 hours and 0 hours. 
A

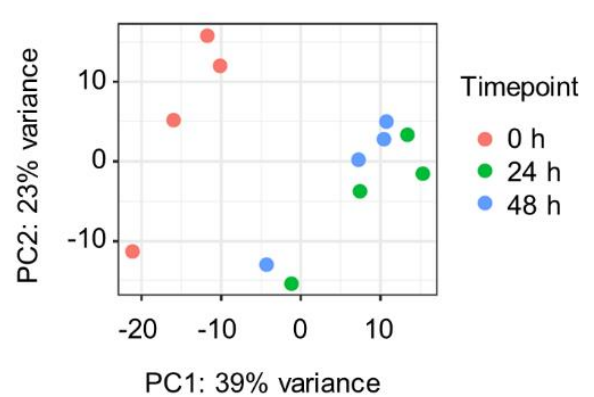

\section{C}

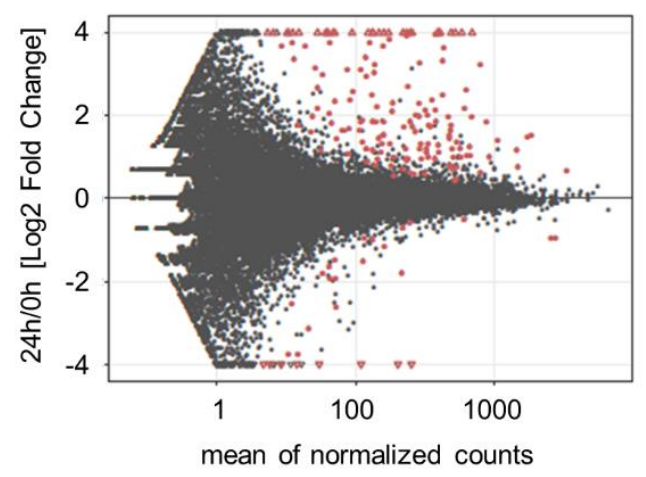

B

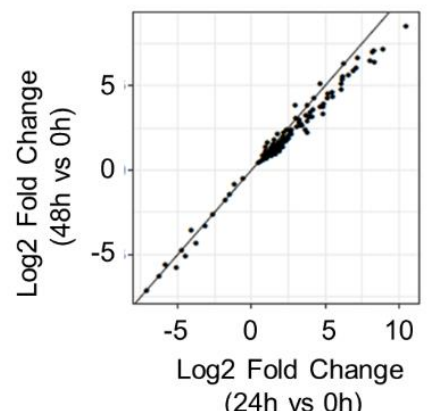

(24h vs $0 \mathrm{~h})$

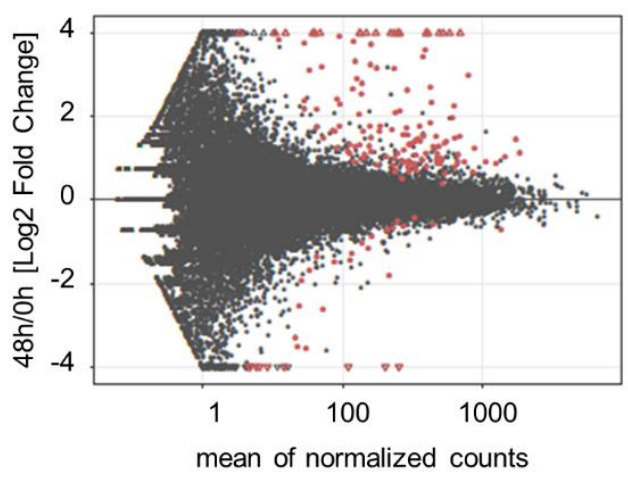

D

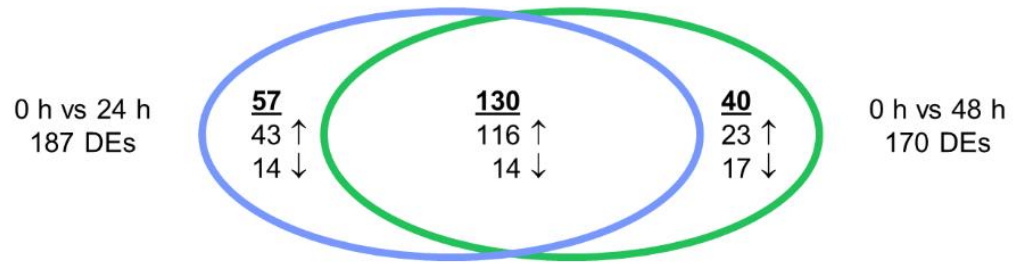

Figure 28: Differential expression analysis shows that most genes are commonly regulated after treatment for $24 \mathrm{~h}$ or $48 \mathrm{~h}$ compared to untreated samples. Figure adapted from Kornstädt et al. (2021), analysis performed by Stefanie Ebersberger and Ingo Ebersberger. (A) PCA shows that the main difference lies between untreated samples $(0 \mathrm{~h})$ and treated samples ( $24 \mathrm{~h}$ and $48 \mathrm{~h}$ ), with a slightly stronger differentiation after $24 \mathrm{~h}$. (B) Log2 fold change of genes significantly regulated in $24 \mathrm{~h}$ vs $0 \mathrm{~h}$ and $48 \mathrm{~h}$ vs $0 \mathrm{~h}$ are very similar with a general stronger response after $24 \mathrm{~h}$. (C) MA plot showing significantly differentially expressed genes in $0 \mathrm{~h}$ vs 24 $\mathrm{h}$ and $0 \mathrm{~h}$ vs $48 \mathrm{~h}$ (adjusted $\mathrm{p}$-value $<0.05$ ). The MA-plots show the log2 fold changes over the mean of normalized counts. Points colored in red represent significantly differentially expressed genes. Points falling out of the displayed plot range (log2 fold change greater than 4 or smaller than -4 ) are plotted as open triangles pointing either up or down. (D) Number of up- and downregulated genes indicated by arrows and total differentially expressed genes (underlined) between $24 \mathrm{~h}$ (blue ellipse) and $48 \mathrm{~h}$ (green ellipse) after induction with zymosan compared to $0 \mathrm{~h}$ untreated samples.

Overall, the PCA indicates that the biggest variation in the data is caused by the treatment of the samples, not by undesired experimental covariates or batch effects, and that the strongest transcriptional changes are observable when comparing the untreated 0 hours samples with the samples treated with zymosan for 24 hours. Next, differential expression between the three groups $(0,24$ and 48 hours) was analysed to discover genes that are up- 
or downregulated after treatment of the cells with zymosan. Differential expression analysis revealed that no genes are significantly differentially expressed between 24 and 48 hours. Meanwhile, when comparing the log2 fold changes between 24 vs 0 hours and 48 vs 0 hours, the response after 24 hours is in general stronger than after 48 hours (Figure 28B). This is in accordance with the PCA, showing the biggest differences between 24 hours and 0 hours (Figure 28A).

As can be seen in the MA-plots, showing the log2 fold changes (M) over the mean of normalized counts of the genes (A), most significantly differentially expressed genes in 24 hours and 48 hours vs 0 hours are upregulated after treatment with zymosan (Figure 28C). In 24 hours vs 0 hours, 187 genes are significantly differentially expressed, in 48 hours vs 0 hours 170 genes are significantly differentially expressed (Figure 28D). 130 genes are collectively regulated in both comparisons, 116 of them being upregulated, 14 of them being downregulated. 57 of the genes that are significantly differentially expressed between 24 hours and 0 hours are not significantly differentially expressed between 48 hours and 0 hours. Out of those 57 genes, 43 are upregulated and 14 are downregulated in 24 hours compared to 0 hours. Vice versa, there are 40 genes that are significantly differentially expressed between 48 hours and 0 hours but not between 24 hours and 0 hours. Out of those, 23 are upregulated and 17 are downregulated in 48 hours compared to 0 hours.

In conclusion, the differential expression analysis reveals that there are no significant differences between 24 hours and 48 hours of treatment and that the strongest response can be seen after 24 hours of zymosan treatment compared to untreated samples. Furthermore, differential expression analysis shows that most differentially expressed genes are upregulated after the zymosan treatment.

\subsubsection{Gene ontology term analysis reveals zymosan-induced type I IFN response in mast cells}

To gain information about the biological processes underlying the significantly differential expressed genes of the mRNA sequencing data, gene set enrichment analysis (GSEA) was performed. GSEA is a method to deduce information about an input gene set by comparing it to background libraries. To run the enrichment analysis, the web-based open source tool Enrichr (Chen et al., 2013; Kuleshov et al., 2016) was used. Enrichr contains mammalian gene set libraries, associated with information on transcription, pathways, ontologies, diseases/drugs, cell types and miscellaneous. Libraries are constructed by assembling gene sets from many sources, like published studies and biological databases. The output of 
Enrichr lists terms linked to annotated gene sets. The resulting lists are ranked according to the level of significant overlap to the input list of genes.

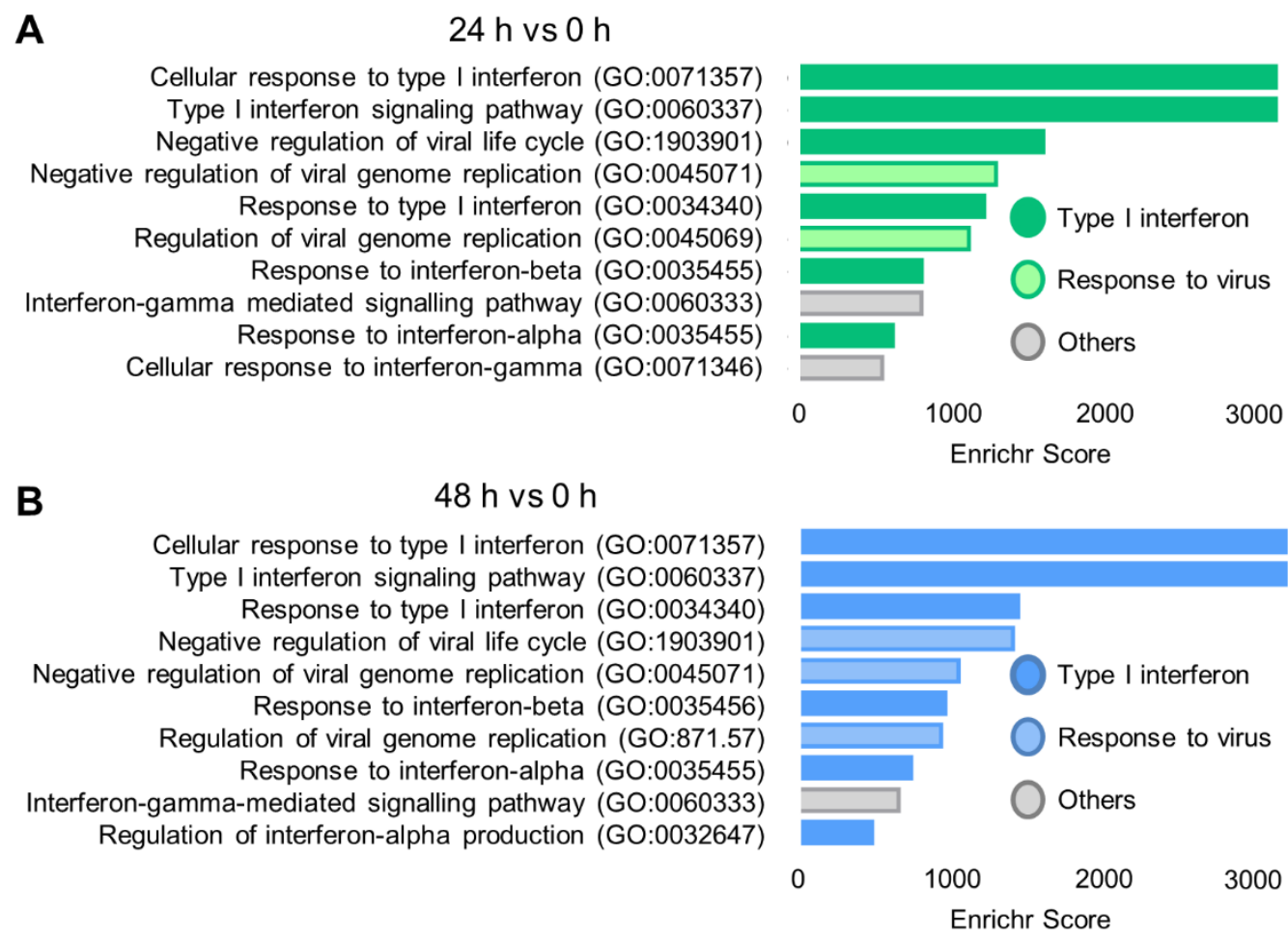

Figure 29: Gene ontology term analysis reveals type I IFN response after induction of BMMCs with zymosan. Figure adapted from Kornstädt et al. 2021. (A) Top ten gene ontology terms of genes upregulated in $24 \mathrm{~h}$ compared to $0 \mathrm{~h}$. (B) Top ten gene ontology terms of genes upregulated in $48 \mathrm{~h}$ compared to $0 \mathrm{~h}$.

Surprisingly, gene ontology term analysis reveals the type I interferon (IFN) pathway as the most prominent response after treatment with zymosan, with 8 and 9 out of the top ten gene ontologies referring to this pathway in genes upregulated in 24 hours vs 0 hours and 48 hours vs 0 hours, respectively (Figure 29A,B). Type I IFNs were first discovered as antiviral peptides and are up to today mostly known for their antiviral functions. In fact, they are pleiotropic cytokines, having anti-tumor and immunoregulatory functions, pro- and antiinflammatory properties, depending on the context (Benveniste \& Qin, 2007; Crow \& Ronnblom, 2019; González-Navajas et al., 2012; McNab et al., 2015; Medrano et al., 2017). The enrichment of type I IFN-related genes after zymosan treatment was highly unexpected, since mast cells are considered unable to produce type I IFNs in response to non-viral TLR activation (Dietrich, Rohde, et al., 2010; Keck et al., 2011).

The type I IFN response generally being regarded as an anti-viral response can explain why some of the genes are assigned to "response to virus" gene ontology terms (Figure 29A,B). The other terms in the list of top ten GO Biological processes are referring to the type II IFN pathway (Figure 29A,B). The only member of the type II IFN family, IFN- $\gamma$, also has effects in 
host resistance to viral and bacterial infections and shares several pathway components and target genes with type I IFNs (Schroder et al., 2004). Furthermore, IFN- $\gamma$ has also been reported to be induced through TLRs (Salerno et al., 2016). The conjoint upregulation of type I and II IFNs is therefore not surprising.

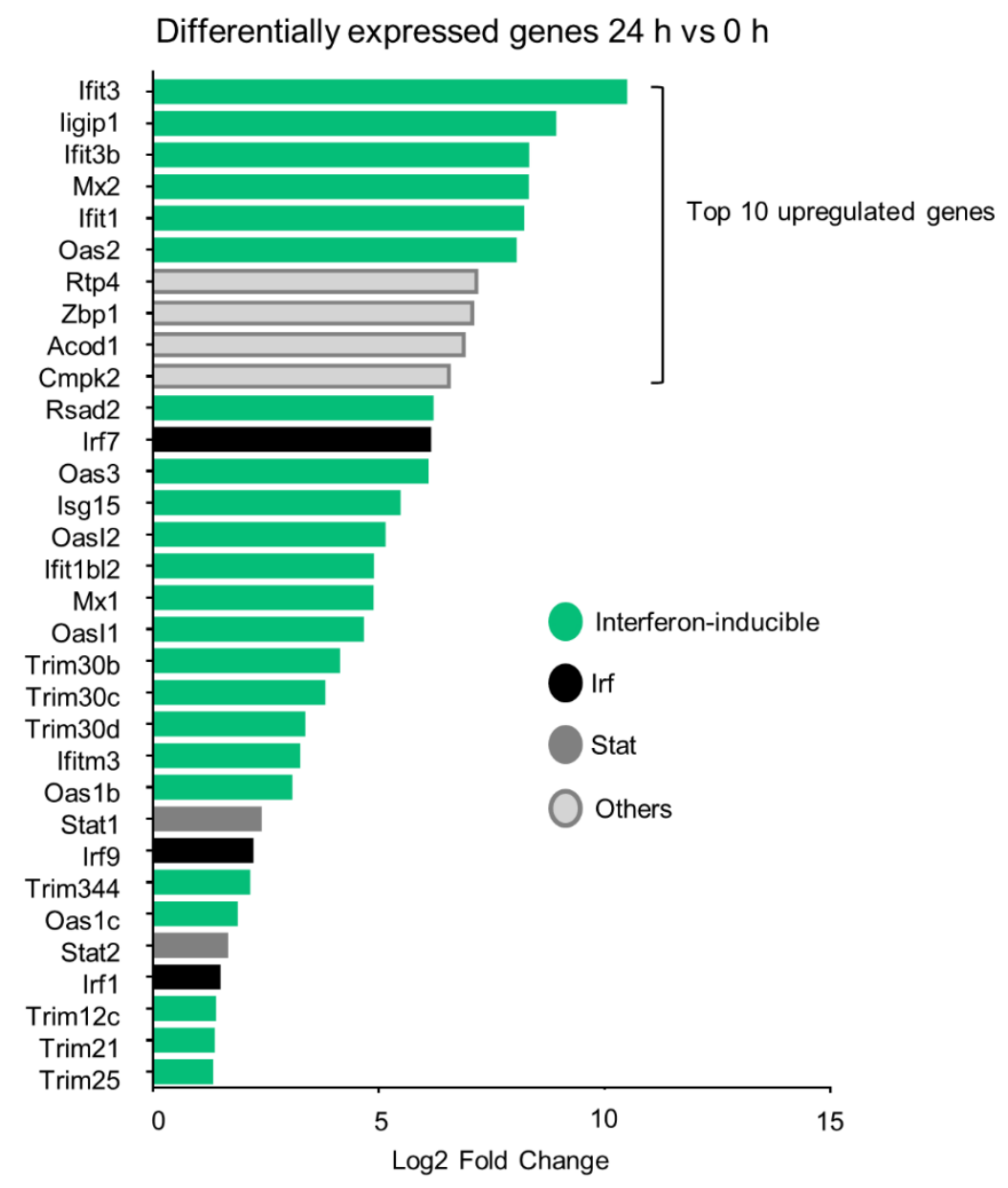

Figure 30: Highly upregulated genes are mostly interferon-inducible. Figure adapted from Kornstädt et al. (2021). List of genes with the strongest upregulation after $24 \mathrm{~h}$ zymosan vs $0 \mathrm{~h}$. Differential expression analysis of untreated $(0 \mathrm{~h})$ BMMCs and BMMCs incubated with $10 \mu \mathrm{g} / \mathrm{ml}$ zymosan for $24 \mathrm{~h}$ before mRNA sequencing.

Looking at the single genes that are differentially expressed after treatment with zymosan shows that most of the strongly upregulated genes are well known IFN-inducible genes (Figure 30), which have been reviewed in Schneider et al. (2014). Rtp4, Zbp1, and Acod1 are also annotated to be induced by interferons (Boys et al., 2020; R. Wu et al., 2020; D. Yang et al., 2020), while Cmpk2 is annotated to be induced by LPS (C. G. L. Lee \& O'Brien, 1995; Lund et al., 2006). Furthermore, the transcription factors Irf7 (6,12 log2 fold increase), Irf9 (2.18 log2 fold increase), Irf1 (1.45 log2 fold increase), STAT1 and STAT2 (2,36 and 1,62 log2 fold increase, respectively) are also among the upregulated genes 24 hours after zymosan treatment. They are known to be present at baseline and to be reinforced by IFN signaling (Borden et al., 2007; Schneider et al., 2014). Irf7, which is one of the strongest 
upregulated genes herein, has been demonstrated to be involved in the induction of type I IFNs by TLR ligands in macrophages (Dietrich, Lienenklaus, et al., 2010). Downregulated genes were associated with general gene ontologies like signal transduction, cell migration, and degranulation.

In conclusion, the RNA sequencing data strongly indicates a type I IFN response evoked in mast cells in response to the TLR ligand zymosan. While mRNA of the cytokines IL-1 $\beta, I L-4$, IL-10, IL-13, and CXCL1, which were identified to be released upon zymosan-induction by ELISA, was also detected, no significant change in their mRNA level was detected in response to the treatment. This might be due to the relatively low number of repeats $(n=4)$ allowing only genes with low variability to reach significance, and due to many cytokines, including IL-4, being pre-stored (Elieh Ali Komi et al., 2020) or regulated post-transcriptionally (Fan et al., 2005). Thus, the type I IFN response appears to be the main response induced 24 hours and 48 hours after incubation with zymosan.

\subsubsection{Validation of type I IFN response in mast cells}

\subsubsection{Mast cells produce IFN- $\beta$ in vitro and in vivo}

Since the mRNA sequencing data strongly indicates a type I IFN response in mast cells, the next aim was to verify this response on the protein level. IFN- $\alpha$ and IFN- $\beta$ are both members of the type I IFN family, which are known to be produced after activation of the transcription factors Irf9, Irf7, and Irf1; therefore, the concentration of IFN- $\alpha$ and IFN- $\beta$ was measured by ELISA after induction with zymosan and LPS.

There are at least 13 different IFN- $\alpha$ subtypes in mouse and human, while there is only one IFN- $\beta$. While in the supernatant of BMMCs induced with zymosan no IFN- $\alpha$ could be detected by an all-subtypes IFN- $\alpha$ ELISA (data not shown), IFN- $\beta$ was detected after induction with both zymosan and LPS (Figure 31A,B). Interestingly, the time course showed a maximum concentration of IFN- $\beta$ already after 6 hours and thus a distinctly earlier release than in the case of IL-10 and IL-1 $\beta$ (Figure 23A,B; Figure 31A,B). IFN- $\beta$ was not detectable in untreated ( 0 hours) mast cells (Figure 31A,B), although it has been described that IFN- $\beta$ is usually present at basal levels in cells (Gough et al., 2012; Hakim et al., 2018; Wang et al., 2017). While basal levels of IFN- $\beta$ might be present, yet too low to be detected, this still suggests that IFN- $\beta$ is not pre-stored in mast cells in high concentration, but newly synthesized upon induction before IL-10 and IL-1 $\beta$ are synthesized. On a further note, the 
amount of IFN- $\beta$ produced by BMMCs is in a similar range as the amount of IFN- $\beta$ synthesized by the same number of bone marrow-derived macrophages (Figure 31C).

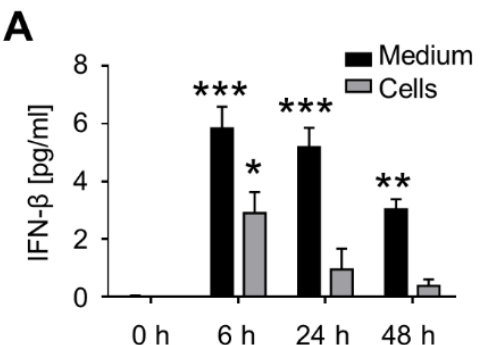

D

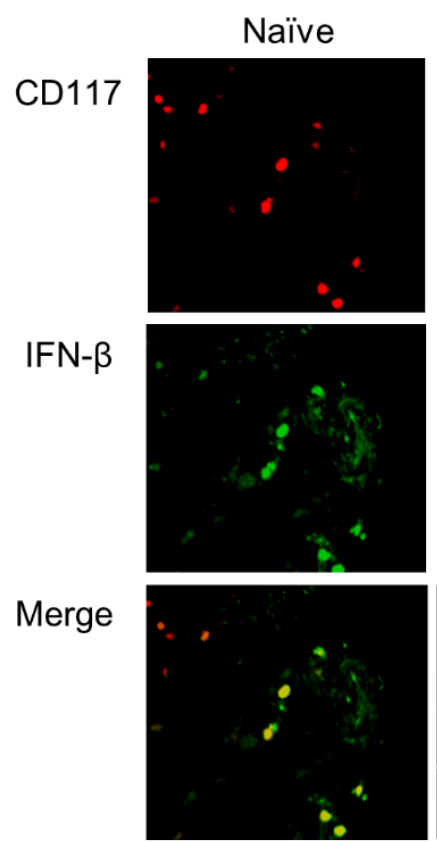

B
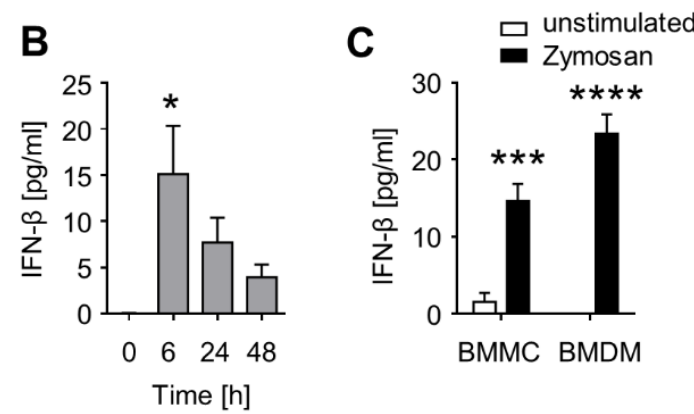

$48 \mathrm{~h}$
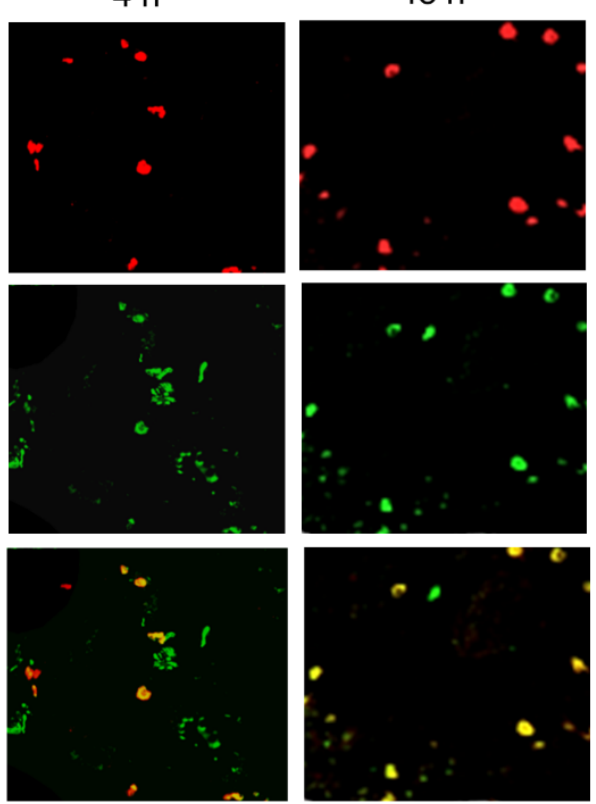

Figure 31: IFN- $\beta$ is produced by mast cells in vitro and in vivo. Figure adapted from Kornstädt et al. (2021). (A) IFN- $\beta$ from BMMCs after induction with zymosan $(10 \mu \mathrm{g} / \mathrm{ml})$ at the indicated time points in cell pellet and supernatant. Data are shown as mean \pm S.E.M. $(n=3)$. One-way ANOVA, Dunnett's multiple comparison test compared to control, ${ }^{*} p<0.05,{ }^{* *} p<0.01,{ }^{* * *} p<0.001$. (B) Release of IFN- $\beta$ from BMMCs after induction with LPS $(100 \mathrm{ng} / \mathrm{ml})$ at the indicated time points. Data are shown as mean \pm S.E.M. $(\mathrm{n}=3)$. One-way ANOVA, Dunnett's multiple comparison test compared to control, ${ }^{*} p<0.05$. (C) Release of IFN- $\beta$ from BMMCs and BMDMs $24 \mathrm{~h}$ after induction with zymosan $(10 \mu \mathrm{g} / \mathrm{ml})$. Data are shown as mean \pm S.E.M. $(n=4)$. Two-way ANOVA, Sidak's multiple comparison test compared to unstimulated control, ${ }^{* * *} p<0.001,{ }^{* * * *} p<0.0001$. (D) Representative stainings for CD117 and IFN- $\beta$ of paws from naïve mice or $4 \mathrm{~h}$ or $48 \mathrm{~h}$ after zymosan-injection ( $\mathrm{n}=3-4)$.

To verify that mast cells can also produce IFN- $\beta$ in vivo, we next performed MELC analysis of paws injected with FITC-zymosan. MELC analysis revealed that mast cells are expressing IFN- $\beta$, not only 4 hours and 48 hours after injection with zymosan, but also in naïve paws (Figure 31D). This seems to be contrary to the results regarding basal storage of IFN- $\beta$ obtained in BMMCs and might indicate for differences between BMMCs and in vivo mast cells. On a further note, mast cells appear to be a major source for IFN- $\beta$ in the tissue at all three time points (Figure 31D). 
The MELC analysis together with the detection of IFN- $\beta$ by ELISA and the RNA sequencing results clearly demonstrate that mast cells are able to produce and release IFN- $\beta$ after induction with zymosan and LPS, contrary to data published by Dietrich, Rohde et al. (2010) and Keck et al. (2011), demonstrating the inability of mast cells to produce type I IFNs in response to TLR4 agonist LPS (Dietrich, Rohde, et al., 2010; Keck et al., 2011).

\subsubsection{IFN- $\beta$ not required for release of IL-10 in mast cells}

One of the known effects of IFN- $\beta$ on immune cells is the involvement in TLR-induced IL-10 production in macrophages (Chang et al., 2007). To elucidate if IFN- $\beta$ is also involved in the release of IL-10 in mast cells in an autocrine feedback loop, BMMCs were treated with IFN- $\beta$ and the concentration of IL-10 in the supernatant was measured 24 hours after induction.
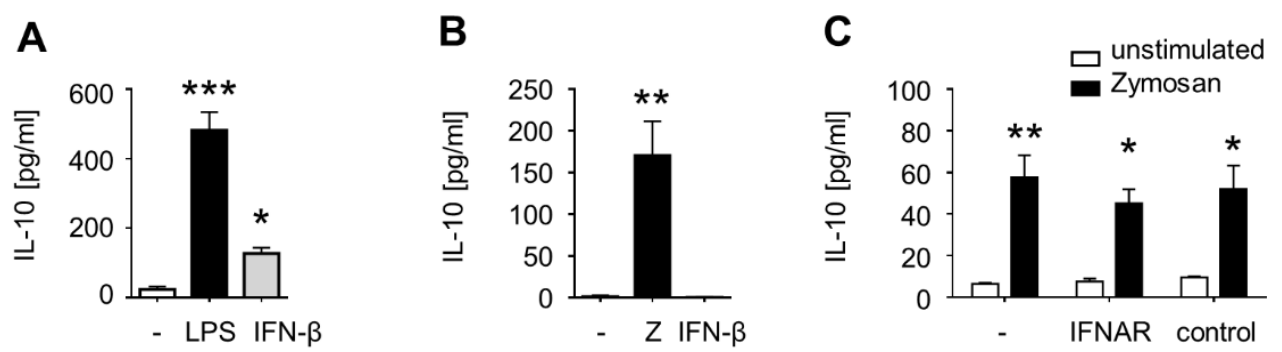

Figure 32: IFN- $\beta$ signaling is not required for release of IL-10 in mast cells. $(A, B) B M D M s ~(p a n e l ~ A)$ and BMMCs (panel B) were induced with $500 \mathrm{U}$ recombinant IFN- $\beta$, and $100 \mathrm{ng} / \mathrm{ml} \mathrm{LPS}$ or $10 \mu \mathrm{g} / \mathrm{ml}$ zymosan $(Z)$, respectively. Concentration of IL-10 was measured $24 \mathrm{~h}$ after stimulation in the supernatant by ELISA. Mean \pm S.E.M. ( $n=4$ for BMDMs, $n=3$ for BMMCs). One-way ANOVA, Dunnett's multiple comparison test, * $p<0.05$, ${ }^{* *} \mathrm{p}<0.01,{ }^{* * * *} \mathrm{p}<0.0001$. (C) BMMCs were pre-treated with $5 \mu \mathrm{g} / \mathrm{ml}$ anti-mouse IFNAR1 antibody, or $5 \mu \mathrm{g} / \mathrm{ml}$ isotope control antibody (Cytochrome C, mouse monoclonal lgG1 kappa), or left untreated, and then stimulated with $10 \mu \mathrm{g} / \mathrm{ml}$ zymosan for $24 \mathrm{~h}$. Concentration of IL-10 in the supernatant was measured by ELISA. Mean \pm S.E.M. (n=3-4). Two-way ANOVA, Tukey's multiple comparison test, ${ }^{*} p<0.05,{ }^{* *} p<0.01$.

The incubation with IFN- $\beta$ induced the release of IL-10 in macrophages as expected, but not in mast cells (Figure 32A,B). Therefore, induction with IFN- $\beta$ does not seem to directly induce the release of measurable concentrations of IL-10 by mast cells. Type I IFNs like IFN- $\beta$ produce their effects by binding to the IFN- $\alpha /-\beta$ receptor subunit 1 (IFNAR1). To rule out that endogenous IFN- $\beta$ or additional signaling through TLR is required for IL-10 production, BMMCs were incubated with anti-IFNAR1 antibody or isotype control antibody and then stimulated with zymosan. No significant difference in the concentration of IL-10 in the supernatant of BMMCs could be detected in anti-IFNAR1 treated cells compared to isotype control treated cells or untreated cells (Figure 32C). 
In conclusion, the release of IL-10 by mast cells after induction with zymosan does not require type I IFN signaling. In contrast, macrophages have been demonstrated to require signaling of the type I IFN pathway for IL-10 induction, demonstrating an anti-inflammatory role of both TLR signaling and type I IFN signaling (Chang et al., 2007). Therefore, the release of IFN- $\beta$ by mast cells might influence neighbouring macrophages and lead to a polarization towards an anti-inflammatory, pro-resolving phenotype, either alone or in combination with other mediators released by mast cells, such as IL-4 and IL-10.

\subsubsection{Release of IFN- $\beta$ by mast cells in response to zymosan and LPS} requires internalization and phagosome maturation

The type I IFN response upon TLR activation is known to require receptor internalization and endosomal acidification (Perkins \& Vogel, 2015). Accordingly, receptor internalization and endosomal acidification has been shown to be a prerequisite of the type I IFN response to bacteria or LPS in macrophages (Dietrich, Lienenklaus, et al., 2010). Mast cells on the other hand are thought to lack this response to non-viral TLR ligands due to being unable to internalize and translocate their respective TLRs into acidic endolysosomal compartments (Dietrich, Rohde, et al., 2010).

Since zymosan is not only recognized by TLR2, but also by Dectin- 1 and nucleotide-binding oligomerization domain 2 (NOD2), these could be alternative pathways to induce the production of IFN- $\beta$, besides the endolysosomal TLR signaling. Furthermore, the Plateletactivating Factor receptor (PAFR) has been shown to be involved in the signaling cascade after zymosan-induction (Guerrero et al., 2013). PAFR is a G-protein coupled receptor localized at intracellular sites like the plasma and the nuclear membrane. The majority of immune cells, including mast cells, are known to express PAFR. Among other pathways, PAFR activates NF- $\mathrm{KB}$ and induces the production of pro-inflammatory mediators (Hills et al., 2019). Dectin-1 is a receptor for fungal $\beta$-glucans and is expressed by mast cells as well as other immune cells (Saijo \& Iwakura, 2011; Taylor et al., 2002; Z. Yang \& Marshall, 2009). Signaling of Dectin- 1 induces the release of several inflammatory cytokines via ERK and NF$\kappa B$, either directly or in interaction with other PRRs (G. D. Brown, 2006; Jia et al., 2014; Reid et al., 2009). NOD2 acts as an intracellular sensor for PAMPs and also induces the transcription of inflammatory mediators. While zymosan does not induce NOD2 directly, cross-talk between TLR2 and NOD2 has been demonstrated upon treatment with zymosan (J. Wu et al., 2015). 
A $\quad \underline{\text { FNN }-\beta}$
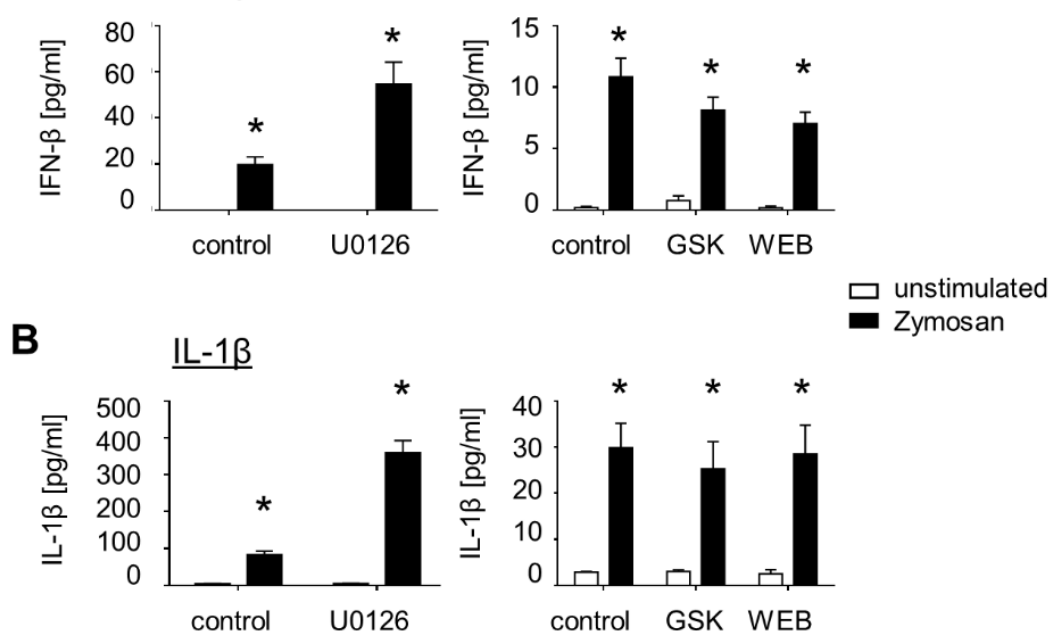

Figure 33: Release of IFN- $\beta$ does not require ERK, NOD2 or PAFR. Figure adapted from Kornstädt et al. (2021). (A) Release of IFN- $\beta$ after pre-treatment with $10 \mu \mathrm{M}$ MEK (ERKI) inhibitor U0126, $10 \mu \mathrm{M}$ NOD2 inhibitor GSK 717, or $10 \mu \mathrm{M}$ PAFR inhibitor WEB 2086 for $1 \mathrm{~h}$ and subsequent treatment with $10 \mu \mathrm{g} / \mathrm{ml}$ zymosan for $24 \mathrm{~h}$. (B) Release of IL-1 $\beta$ after pre-treatment with $10 \mu \mathrm{M}$ U0126, GSK 717 or WEB 2086 for $1 \mathrm{~h}$ and subsequent treatment with $10 \mu \mathrm{g} / \mathrm{ml}$ zymosan for $24 \mathrm{~h}$. Data is shown as mean \pm S.E.M. $(n=4)$. Multiple t-test comparing unstimulated and zymosan stimulated cells, Holm Sidak's multiple comparison test, ${ }^{*}=$ significant.

To analyse if any of these receptors are inducing the type I IFN response, mast cells were pre-treated with the ERK inhibitor U0126, the NOD2 inhibitor GSK 717, or the PAFR inhibitor WEB 2086 before treating the cells with zymosan. The concentrations of the inhibitors were chosen according to literature or manufacturer's instructions (Ajiro et al., 2011). Mast cells treated with these inhibitors are still producing IFN- $\beta$, as well as the pro-inflammatory cytokine IL-1 $\beta$ after zymosan induction (Figure 33A,B). Intriguingly, inhibition of the ERK pathway with $\mathrm{U} 0126$ induced a stronger release of both IFN- $\beta$ and IL-1 $\beta$. This may be explained by the dual nature of ERK-signaling, which has been reported to be able to switch form inhibiting to stimulating the transcription of cytokines (Hedl \& Abraham, 2012). In conclusion, neither inhibition of ERK-, nor NOD2- or PAFR-signaling were able to abrogate the production of IFN- $\beta$. Since TLR signaling is known to induce transcription of cytokines in response to zymosan, the release of IL-1 $\beta$ was not reduced by the inhibitors used herein as expected. While a role of the ERK, NOD2 and PAFR in the induction of IFN- $\beta$ can not be completely ruled out due to a lack of more specific positive controls for the inhibitors, all three inhibitors having no limiting effect is at least a strong hint that they don't play an important role in the induction of IFN- $\beta$.

Mast cells have so far been considered unable to produce IFN- $\beta$ in response to non-viral TLR ligands, due to their proposed inability to internalize the receptor and due to the absence of TRIF signaling, as shown by Dietrich, Rohde, et al., (2010) and Keck et al., (2011). In this study, the ability of mast cells to elicit a type I IFN response upon induction 
with zymosan and LPS was clearly demonstrated. Meanwhile the involvement of an alternative pathway including ERK, NOD2, and PAFR could not be observed. Actin-mediated receptor internalization and endosome acidification is known to be a pre-requisite for TLRmediated induction of the type I IFN response. To investigate if receptor internalization and endosome acidification are a pre-requisite for TLR-mediated induction of the type I IFN response in mast cells as well they were pre-treated with Cytochalasin $D$, an inhibitor of actin polymerization, and Bafilomycin A, a proton pump inhibitor, before addition of zymosan or LPS. Since IL-1 $\beta$ is known to be induced by TLR signaling from the cell membrane and not from endosomal compartments, the release of $\mathrm{IL}-1 \beta$ served as the positive control. The release of IFN- $\beta$ by mast cells was impaired by either of the inhibitors, while the proinflammatory response (IL-1 $\beta$ release) was not reduced (Figure 34A,B). Thus, mast cells require the same mechanisms of internalization and endosomal acidification for the induction of the IFN- $\beta$ response that have been shown to be necessary for macrophages previously (Dietrich, Lienenklaus, et al., 2010), while the pro-inflammatory response is induced independently of these mechanisms, just like in macrophages.

A $\quad \underline{\text { IFN }-\beta}$
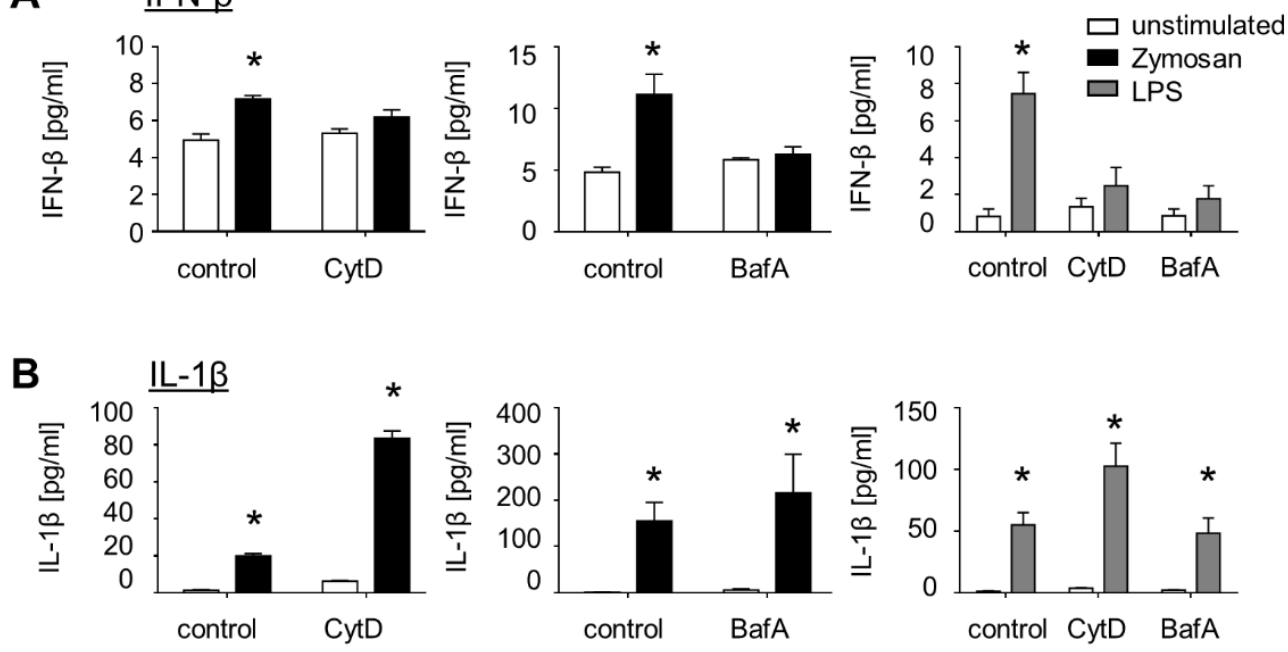

Figure 34: Release of IFN- $\beta$ by mast cells requires receptor internalization and endosome maturation. Figure adapted from Kornstädt et al. (2021). BMMCs either untreated or pre-treated for $1 \mathrm{~h}$ with $25 \mu \mathrm{M}$ Cytochalasin D or $50 \mu \mathrm{M}$ Bafilomycin A were stimulated for $24 \mathrm{~h}$ with $10 \mu \mathrm{g} / \mathrm{ml}$ zymosan or $100 \mathrm{ng} / \mathrm{ml}$ LPS. The concentrations of IFN- $\beta$ (panel A) or IL-1 $\beta$ (panel B) were determined in the medium. Data is shown as mean \pm S.E.M. $(n=4)$. Multiple t-test comparing unstimulated and zymosan or LPS stimulated cells, Holm Sidak's multiple comparison test, ${ }^{*}=$ significant.

In summary, besides identification of the type I IFN pathway as a novel response in mast cells to bacterial and fungal stimuli and besides showing that mast cells are located in an anti-inflammatory region of the tissue during resolution inflammation, we demonstrated the enhancing effect of mast cells on the phagocytic and efferocytic activity of macrophages, thereby contributing to the resolution of inflammation. 


\section{Discussion}

Being located closely to surfaces, which are exposed to the environment, mast cells are one of the first immune cells that come into contact with invading pathogens and other harmful stimuli. Their various immunomodulatory functions root in their ability to respond to a wide range of stimuli by releasing specific subsets of diverse mediators (J. R. Gordon et al., 1990). While their roles in initiating and promoting immune responses are well described, potential anti-inflammatory and immunosuppressive functions are still under discussion and have been mostly assigned to the cytokine IL-10 (Chan et al., 2013; Depinay et al., 2006; Galli et al., 2008; Grimbaldeston et al., 2007; Reber et al., 2017).

In this thesis, the phenotype of mast cells and their effect on their surroundings was analysed to obtain a broader understanding of their role during the resolution of inflammation. Therefore, first of all their location in the inflamed tissue in regard to the source of inflammation and to neighbouring cells was analysed. Furthermore, the effect of mast cells on their neighbouring cells was analysed by MELC and polychromatic flow cytometry of immune cells in inflamed paws in mast cell-deficient mice and control mice. Finally, an untargeted approach to identify mediators produced and pathways activated during the resolution phase was performed.

\subsection{Comparison of two locally induced inflammation models}

Before analysing the role of mast cells in resolution, a suitable model of a local inflammation needed to be identified. Therefore, the inflammatory responses raised by the two TLR agonists LPS (TLR4) and zymosan (TLR2) were compared, since both are commonly used for the induction of inflammation. The data indicated that, while the chosen concentration induced an inflammation with comparable duration and intensity, LPS additionally induced an early systemic inflammation (Hahnefeld \& Kornstädt et al., 2021).

Analysis of lipid profiles in ipsilateral and contralateral paws revealed that both LPS- and zymosan-injection caused elevated levels of lipids in the inflamed tissue, while only LPSinjection but not zymosan-injection induced significant changes in contralateral paws. Among the upregulated lipids in LPS- and zymosan-injected ipsilateral paws are etherphosphatidylcholines (PC O) and their lyso species (LPC O), which are discussed to act as antioxidants protecting the plasma membrane against ROS released during the inflammatory processes (Skaff et al., 2008). In the contralateral paws of LPS-injected mice, especially phosphatidylcholines (PC) and phosphatidylethanolamines (PE) and their ether species were 
selectively upregulated. These have been shown to be associated with mitochondrial complexes and might therefore reflect an increased mitochondrial energy production in response to increased energy consumption through inflammatory processes on the contralateral side (Acoba et al., 2020; Calzada et al., 2019; Guo et al., 2017; Lange et al., 2001). Furthermore, levels of several cytokines and chemokines and one growth factor were increased in the plasma of mice injected with LPS, but not in mice injected with zymosan. Increased cytokine and chemokine levels in the plasma are associated with the acute phase response in systemic inflammation (Bernotiene et al., 2004; Cheng et al., 2018). This indicates that LPS induces a widespread systemic effect, while zymosan induces a local inflammation. Meanwhile, the injection of zymosan induced the formation of an edema in contrast to LPS-injection. Accordingly, higher levels of infiltrating immune cells were detected in the ipsilateral paws of zymosan-injected mice compared to LPS-injected mice. Taken together, these data demonstrate a higher accumulation of leukocytes and plasma to the site of inflammation in the zymosan model compared to the LPS model.

The stronger local reaction towards zymosan and the systemic response to LPS might be explained by the different solubility of the two reagents. While a soluble solution can be prepared with LPS, zymosan particles are insoluble. Therefore, zymosan particles need to be cleared from the tissue by phagocytosis, while LPS can probably be easily absorbed and may also be distributed in the body, causing the observed systemic inflammatory response. Besides causing a strong local inflammation zymosan also has the advantage that it can be visualised in the tissue by labelling it with FITC before intraplantar injection. Thereby, analysis of the location of immune cells in regard to zymosan can be analysed by MELC, showing the architecture of the inflammation. As a result, zymosan was chosen as the most suitable model to study the role of mast cells during the resolution of inflammation in this thesis.

\subsection{Anti-inflammatory microenvironment of mast cells}

Mast cells are tissue resident cells and part of the first line of defence of the body against invading pathogens and other harmful stimuli (Erb et al., 1996). In response to the detection of harmful stimuli, more immune cells are recruited to the site of inflammation. The different types of immune cells are specialized for distinct and partly overlapping functions. They communicate and influence each other via the release of mediators, affecting their phenotype and functionality, and the progression of the inflammation. Thus, being embedded in this network of cells, mast cells are influencing as well as being influenced by their direct 
surrounding. In order to gain information on their phenotype as well as on their effect on the resolution of inflammation, it is crucial to know the environment of mast cells.

The receding mechanical hypersensitivity after injection of $12 \mathrm{mg} / \mathrm{ml}$ zymosan into the paw served as indication for the resolution phase of the inflammation and was thus chosen as the time point to study the role of mast cells during resolution of inflammation. MELC analysis of naïve paws and paws during the peak of the inflammation mirrored the typical course of an inflammation, characterised by infiltration of neutrophils $\left(\mathrm{Ly} 6 \mathrm{G}^{+}\right)$, followed by infiltration and polarisation of macrophages $\left(\mathrm{F} 4 / 80^{+}\right)$(Serhan et al., 2007). Analysing cellular neighbourhoods using MELC images from FITC-zymosan-injected paws during resolution of inflammation revealed that mast cells are located in an anti-inflammatory environment next to M2 macrophages, surrounding the zymosan-filled area dominated by pro-inflammatory M1 macrophages $\left(\mathrm{CD} 86^{+}\right)$and neutrophils 48 hours after zymosan-injection.

Although recruitment of mast cell progenitors to sites of inflammation (Bot et al., 2015; Collington et al., 2011), as well as the potential of mature mast cells to proliferate in vivo (S. Sonoda et al., 1986; T. Sonoda et al., 1984) have been demonstrated, we could not observe a significant increase of mast cell numbers in inflamed tissue by flow cytometry analysis of whole paw lysates. However, we found a tendency towards increased mast cell numbers during the peak of inflammation compared to naïve paws and we can not exclude a local increase of mast cell numbers at the site of inflammation.

Polarisation of resident macrophages and infiltrating monocytes towards an M1 phenotype is induced by noxious stimuli and leads to the production and release of pro-inflammatory mediators, and reactive oxygen and nitrogen intermediates, resulting in killing of pathogens and promotion of inflammation (Mantovani et al., 2004). Therefore, the location of M1 macrophages in the center of the inflammation is in accordance with their functions. Like M1 macrophages, neutrophils are also located in the zymosan-filled area, which fits to their role as the prototypical phagocytic cells (Silva \& Correia-Neves, 2012). Remarkably, neutrophils in the zymosan-filled area show a stronger expression of CD11b than neutrophils that do not co-localize with zymosan. The receptor CD11b, also known as CR3 or $\alpha_{M} \beta_{2}$, is a known mediator of phagocytosis, underlining the phagycytosing activity of the neutrophils in the core region of the inflammation (Cougoule et al., 2004). Meanwhile M2 macrophages are known to be involved in the repair of inflammation-induced injury (Lech \& Anders, 2013), fitting to their location right at the border to the core of the inflammation.

The division of the tissue into pro- and anti-inflammatory regions shows how precisely orchestrated the immune response is. While the destruction of potential invaders and the phagocytosis of zymosan particles are probably the main tasks in the pro-inflammatory core region of the inflammation, one can imagine various functions of the anti-inflammatory region 
surrounding the core of the inflammation. First of all, it needs to be ensured that the inflammatory processes are limited to the affected tissue in order to protect the surrounding tissue. This can either be achieved by mediators gradually losing their potency with growing distance to their source, or by an anti-inflammatory barrier, shielding the surrounding tissue from pro-inflammatory influences, or a combination of both. Furthermore, regeneration of the damaged and previously inflamed tissue, efferocytosis of dead cells, and return to homeostasis are probably the main functions of the anti-inflammatory region observed here. Therefore, the architecture of the inflammation shows that the onset and the resolution of a local inflammation can not only be considered as a timely resolved, but also has to be considered as a spatial resolved mechanism. These aspects have also been considered in mathematical models of the resolution of inflammation before (Bayani et al., 2020).

In conclusion, the accumulation of mast cells in the area surrounding the core of the inflammation and their cellular environment suggests a potential anti-inflammatory, proresolving role of mast cells during the resolution of inflammation. Furthermore, analysis of the environment of mast cells in the inflamed tissue enabled the identification of neighbouring cells, which are possibly influencing mast cells or being influenced by them. Since neighbourhood analysis showed M2 macrophages in close proximity of mast cells during resolution of inflammation, the effect of mast cells on the phenotype of macrophages was analysed next.

\subsection{Pro-resolving effect of resolution phase mast cells}

PhenoGraph analysis as well as polychromatic flow cytometry showed a decreased uptake of neutrophils and zymosan by macrophages in mast cell-deficient mice. Meanwhile the number of neutrophils, monocyte-derived macrophages, eosinophils, and dendritic cells present in the inflames tissue was not affected by mast cell-deficiency, demonstrating that, albeit mast cells are known to be involved in the recruitment of neutrophils in the onset of the inflammation (De Filippo et al., 2013), they do not play a dominant role in the numbers of immune cells present during resolution, and furthermore the decreased uptake of neutrophils by macrophages is not due to a decreased number of neutrophils at the site of inflammation. Also, the effect on phagocytosis and efferocytosis was not due to a shift in macrophage polarization between M1 and M2 phenotypes, since no changes in M1 and M2 macrophage numbers could be observed. This is in accordance with previous data, demonstrating the ability of mast cells to enhance neutrophil clearance by macrophages without affecting their level of the M2 marker CD206 (Jachetti et al., 2019), which is known to function in phagocytosis and efferocytosis (Allavena et al., 2004; S. J. Lee et al., 2002). 


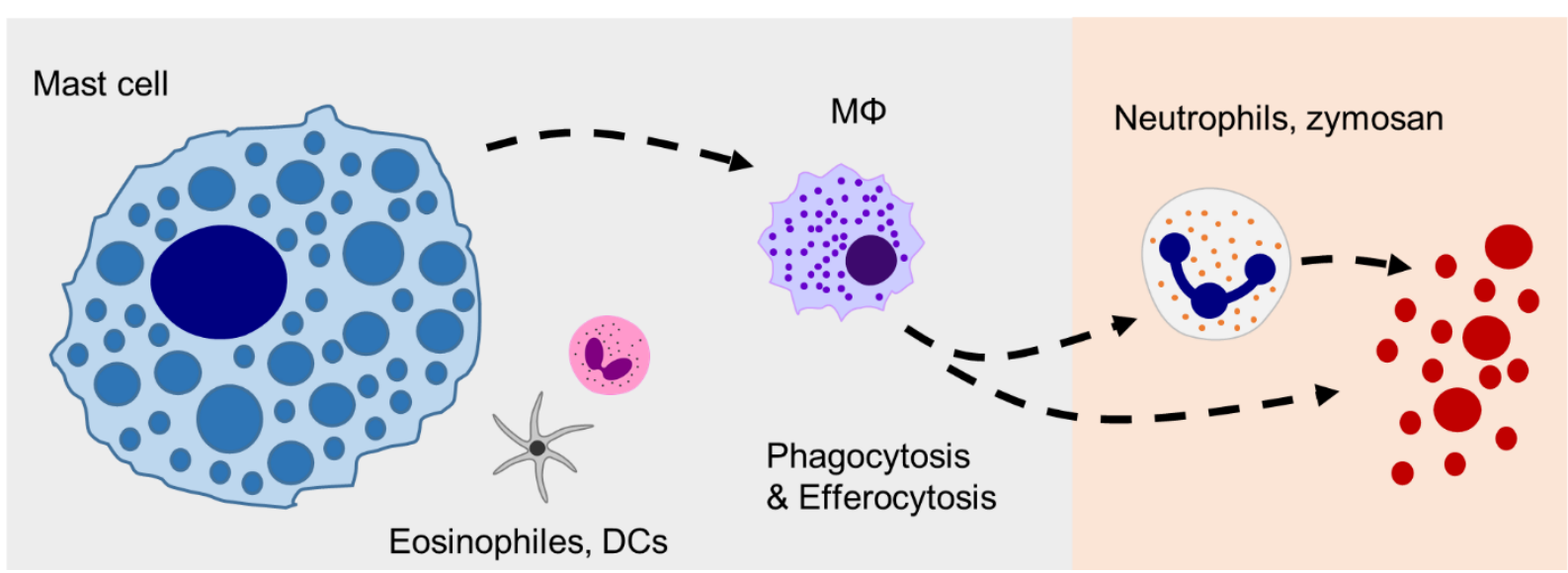

Figure 35: Mast cells are located in anti-inflammatory neighbourhood and influence phagocytosis and efferocytosis activity of macrophages during resolution of inflammation. DCs = dendritic cells.

Jachetti et al. (2019) demonstrated that mast cells enhance the efferocytic activity of macrophages both in vitro and in vivo using mast cell deficient C57BL/6 $\mathrm{Kit}^{\mathrm{W}-\mathrm{sh}}$ mice. This mouse strain is characterized among other abnormalities by a pronounced neutrophilia (Nigrovic et al., 2008), which could be selectively rescued by reconstitution with in vitrodifferentiated mast cells. An effect on neutrophil maturation, survival, and functionality, as well as a direct role of mast cells in clearance of neutrophils was ruled out. Furthermore, they showed that accumulation of neutrophils in C57BL/6 Kit ${ }^{\mathrm{W} \text {-sh }}$ mice is not due to diminished numbers of macrophages, and they observed an increase in the expression of Tim4 in macrophages, which is an essential receptor of macrophages for phagocytosis (Nishi et al., 2014). By analysing the intracellular expression of the neutrophil marker Ly6G in macrophages, they assessed the capacity of macrophages to engulf neutrophils, which was significantly decreased in mast cell-dificient C57BL/6 Kit ${ }^{\mathrm{W} \text {-sh }}$ mice and restored in mast cellreconstituted $\mathrm{C} 57 \mathrm{BL} / 6 \mathrm{Kit}^{\mathrm{W} \text {-sh }}$ mice. Flow cytometry analysis of $\mathrm{APC}$-labelled neutrophils in macrophages co-cultured with BMMCs confirmed the ability of mast cells to enhance neutrophil clearence by macrophages in vitro.

To confirm the effect of mast cells and to exclude the contribution of other Kit-dependent phenotypic differences a common approach has been to reconstitute Kit-dependent mast cell-deficient mice with in vitro-differentiated mast cells. However, there are several caveats to this experimental approach. First of all, since Kit is expressed by a variety of hematopoietic stem cells during their early development, some effects on myeloid cells in C57BL/6 Kit ${ }^{\mathrm{W}-\mathrm{sh}}$ are probably irreversible and might influence the outcome of an experiment. Furthermore, transfer of in vitro-differentiated mast cells does not result in physiological levels and physiological tissue distribution of mast cells (Nakano et al., 1985). Therefore, results obtained by reconstituted C57BL/6 Kit ${ }^{\mathrm{W}-\text { sh }}$ have to be interpreted carefully. Indeed, many results regarding mast cell effects obtained in Kit-dependent mouse models could not 
be reproduced in Kit-independent mouse models (Feyerabend et al., 2016; Rodewald \& Feyerabend, 2012).

Jachetti et al. (2019) state themselves that the role of mast cells in regulating neutrophil clearance by macrophages requires further studies to demonstrate the significance of this result beyond their observations in C57BL/6 Kitw-sh mice. While Kit-dependent mast celldeficiency as in C57BL/6 Kit ${ }^{\mathrm{W} \text {-sh }}$ mice is characterized by phenotypic abnormalities beyond mast cell-deficiency, including expanded frequencies of neutrophils (Nigrovic et al., 2008), Mcpt5-DTA Cre mice have no known hematological abnormalities (Dudeck et al., 2011). Taken together, the data presented by Jachetti et al. (2019) and the data presented herein demonstrate the ability of mast cells to influence the phagocytic activity of macrophages.

Efferocytosis of apoptotic cells is a known prerequisite for the successful resolution of an inflammation (Kourtzelis et al., 2020; Maderna \& Godson, 2003; Savill et al., 2002). Fittingly, neutrophils were more present during peak inflammation 4 hours after zymosan-injection than during resolution phase 48 hours after zymosan-injection as observed by MELC analysis, while macrophages positive for the neutrophil marker Ly6G were more present during the resolution phase, reflecting the increased efferocytosis during this phase. Therefore, mast cells have the potential to contribute to the resolution of inflammation by enhancing efferocytosis by macrophages (Figure 35).

\subsection{Phenotype of mast cells during resolution of inflammation}

Mast cells are equipped with secretory granules densely packed with pre-formed mediators that can be released rapidly by degranulation. In addition to the release of pre-formed mediators, activation of mast cells can also induce de novo synthesis and release of lipid mediators and chemokines. The large repertoire of receptors of mast cells enables them to discriminate between various stimuli and precisely respond with a selective release of mediators. Furthermore, mast cells are considered to adjust their response during different phases of the progressing inflammation, contributing either to the promotion or the resolution of the inflammation (Galli et al., 2008).

While the list of known mast cell products has been ever growing, their anti-inflammatory functions have been mostly assigned to the cytokine IL-10 (Chan et al., 2013; Depinay et al., 2006; Grimbaldeston et al., 2007; Reber et al., 2017). To identify mast cell-derived mediators which might directly or indirectly affect the resolution of inflammation and enhance macrophage-mediated phagocytosis, the levels of a range of cytokines, chemokines and 
growth factors associated with inflammatory processes were analysed in mast cell-deficient Mcpt5-DTA Cre mice.

Besides the reduced clearance of neutrophils by macrophages, Jachetti et al. (2019) present a second potential mechanism that could lead to increased neutrophil numbers in C57BL/6 $\mathrm{Kit}^{\mathrm{W}-\mathrm{sh}}$ mice. They observed an increase of G-CSF and IL-17 in the sera of mast cell-deficient C57BL/6 Kit ${ }^{\mathrm{W}-s \mathrm{~h}}$ mice, which act in a complex network with other cytokines as major drivers of neutrophil proliferation (Bugl et al., 2012). In accordance with unaltered neutrophil numbers in the inflamed paw assessed by flow cytometry in this thesis, we could not detect a significant change of G-CSF and IL-17 in the inflamed paw of mast cell-deficient Mcpt5-DTA $\mathrm{Cre}^{+}$mice. Therefore, while G-CSF and IL-17 might play an additional role in the regulation of neutrophil numbers during homeostasis, as investigated by Jachetti et al. (2019), they do not seem to be involved in the regulation of neutrophils numbers during resolution of inflammation investigated herein. Furthermore, differences in the levels of G-CSF and IL-17 within C57BL/6 Kit ${ }^{W}$-sh might be due to Kit-deficiency, not due to mast cell-deficiency.

Among the other mediators analysed, only IL-4 and CXCL1 were significantly decreased in the inflamed paw in the absence of mast cells. The ability of mast cells to release IL-4 and CXCL1 was confirmed in vitro by stimulating BMMCs with LPS or zymosan. CXCL1 is known to recruit neutrophils towards the site of infection during the onset of an inflammation (De Filippo et al., 2013). Since neutrophil numbers are not reduced in the absence of mast cells, other functions of CXCL1 might play a role during this late stage of the inflammation, i.e. its role in driving epithelialization and angiogenesis in wound healing (Castela et al., 2017), which fits better to the context of resolution of inflammation. IL-4 is a prototypical antiinflammatory cytokine, driving polarization of macrophages towards an M2 phenotype, but no shift in macrophage phenotypes could be observed by flow cytometry. Meanwhile, another function of IL-4 that has been reported is its ability to increase phagocytic activity of macrophages (Daseke et al., 2020; Wainszelbaum et al., 2006). IL-13, another cytokine produced by mast cells in response to zymosan and LPS in vitro, is also known for its role in enhancing efferocytosis and phagocytosis (Korns et al., 2011). Together with IL-4, IL-13 leads to the increased expression and activity of the nuclear receptor PPARY via STAT6 in macrophages (Berry et al., 2007; Szanto et al., 2010; Welch et al., 2003), which in turn leads to an increase in efferocytic surface receptors, enhanced efferocytic capability, and suppression of inflammation.

In an untargeted approach to identify a more complete set of mediators induced in mast cells upon zymosan-induced TLR signaling, mRNA sequencing was performed and surprisingly revealed a strong type I IFN response in mast cells. Previously, mast cells have been demonstrated to be unable to produce type I IFNs in response to non-viral TLR stimuli by 
Keck et al. (2011) and Dietrich, Rohde et al. (2010). Contradicting these previous studies, mast cells released IFN- $\beta$ in response to both zymosan and LPS in this study. While Keck et al. (2011) used L929 cells transfected with an IFN-sensitive luciferase construct to measure IFN- $\alpha /-\beta$, Dietrich et al. (2010) determined the production of IFN- $\beta 6$ hours after induction of the cells by RT-PCR. As for the absence of IFN- $\beta$ in the study of Keck et al. (2011), IFN- $\beta$ was also not detected after viral induction of mast cells by the L929 cells transfected with an IFN-sensitive luciferase construct used by them, although mast cells are known to produce type I IFNs upon viral induction (Marshall et al., 2019). This leads to the conclusion that direct measurement by ELISA as used in this thesis might be more suitable to detect IFN- $\beta$ than indirect measurement as by IFN-inducible Luciferase-producing cells used by Keck et al. (2011). Meanwhile Dietrich et al. (2010) proposed that the lack of IFN- $\beta$ produced by mast cells in response to non-viral TLR induction is due to their inability to internalize TLR-receptor ligand complexes. This is in contrast to other studies, demonstrating the ability of mast cells to internalize TLR receptors, for example after LPS induction (Pérez-Rodríguez et al., 2020), but also in other contexts (Meng et al., 2013; Okumura et al., 2003).

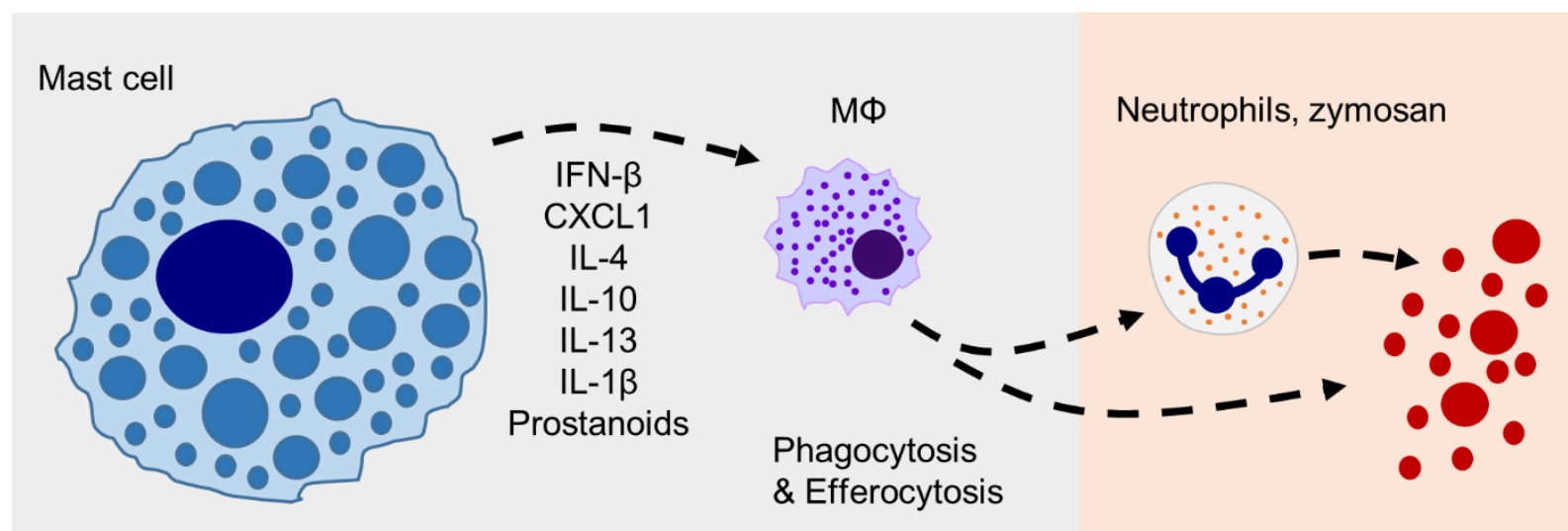

Figure 36: Mast cells release a plethora of mediators in response to TLR activation, of which some have been shown to influence the phagocytosis and efferocytosis activity of macrophages.

In line with previous studies demonstrating that TLR signaling from endolysosomal compartments is required for the induction of type I IFNs (Perkins \& Vogel, 2015), the type I IFN response was abrogated after inhibition of receptor internalisation and endosomal maturation, while the pro-inflammatory response initiated from cell-surface located TLRs is still active. Furthermore it was shown that TRAF3, which is necessary for the induction of type I IFNs (Oganesyan et al., 2006), is only recruited to endosomal membranes, not to the plasma membrane (Bauernfeind \& Hornung, 2009; Kagan et al., 2008; Perkins \& Vogel, 2015). Keck at al. (2011) explained the inability of mast cells to induce type I IFNs by the absence of TRIF signaling. Induction of type I IFNs by TLR4 has been shown to be mediated by TRIF (Kagan et al., 2008), TLR2 induction of IFN- $\beta$ from endolysosomal compartments on 
the other hand has been shown to depend on MyD88 and TRAM, but not TRIF, and to be mediated by IRF7 just like endosomal IRF7 activation mediated by MyD88 upon TLR9 signaling (Barbalat et al., 2009; Bauernfeind \& Hornung, 2009; Stack et al., 2014). Since TLR4 is also associated with MyD88, type I IFNs could also be induced by MyD88-mediated TLR4 signaling in mast cells. On this note, it has already been shown in other cell types that intracellular location does not necessarily lead to TRIF-TRAM-dependent signaling, but is also required for the MyD88-dependent pathway (Shibata et al., 2011).

Time course analysis showed that IFN- $\beta$ is released by BMMCs much earlier than IL-10 and $\mathrm{IL}-1 \beta$, with maximal concentrations of IFN- $\beta$ detected in the supernatant of BMMCs already after 6 hours and for IL-10 and IL-1 $\beta$ after 24 hours. IFN- $\beta$ was not detected in unstimulated cells themselves, demonstrating that IFN- $\beta$ synthesis is induced by stimulation of the cells with LPS and zymosan rather than being pre-stored. The observed time frame indicates for a direct induction of IFN- $\beta$ through TLR activation, rather than the involvement of intermediate mediators. This is in accordance with previous studies, demonstrating the direct induction of IFN- $\beta$ by TLR2 (Dietrich, Lienenklaus, et al., 2010).

Importantly, IFN- $\beta$ was detected in naïve paws in vivo by immunohistochemistry, indicating the storage of IFN- $\beta$ under basal conditions rather than complete de novo synthesis in contrast to the in vitro situation analysed in BMMCs. Meanwhile, IFN- $\beta$ was not detectable by ELISA in vivo. Since mast cells are relatively rare cells in the tissue, IFN- $\beta$ levels might be too low to be detected in whole paw lysates even if they are increased locally. In this regard, also the release of $\mathrm{IL}-10, \mathrm{IL}-13$ and $\mathrm{PGE}_{2}$ by mast cells was detected in vitro, while no alterations regarding their levels were detected in paw lysates of Mcpt5-DTA Cre mice. This could also be due to the presence of further immune cells in the tissue with the capacity to contribute to the concentration of these mediators, masking mast cell-derived changes. Importantly, other cells contributing to the overall concentration of these mediators in the whole paw does not oppose potential effects of these mast cell-derived mediators on neighbouring cells.

One possible explanation for the different in vitro and in vivo situation regarding the storage of basal IFN- $\beta$ could be phenotypical differences between BMMCs in cell culture and mast cells residing in the microenvironment of the tissue. In general, it is agreed upon that IFN- $\beta$ is expressed by cells constitutively (Gough et al., 2012). Meanwhile, basal IFN- $\beta$ levels are known to be close to detection thresholds even of sensitive assays (Hamilton et al., 1996; Vogel \& Fertsch, 1984). Therefore, IFN- $\beta$ might still be present under basal conditions in BMMCs in levels below the detection level of the ELISA, while the specific microenvironment of mast cells in the paw tissue might allow for storage of higher levels of basal IFN- $\beta$. 
Type I IFNs have been originally described as anti-viral and are even used as a target for treatment of some viral infections and autoimmune diseases (Crow \& Ronnblom, 2019; Li et al., 2018). Beyond their function in limiting virus replication and initiating an antiviral immune response, they also have immunosuppressive functions, thereby minimizing immunopathology (A. J. Lee \& Ashkar, 2018). This dual nature of type I IFNs also applies to its role in non-viral infections. First evidence on the role of type I IFNs in microbial infections was discovered more than 50 years ago, but has been appreciated only relatively recently (Bogdan et al., 2004; Sueltenfuss \& Pollard, 1963). The antiviral activity of type I IFNs is mostly derived from effector proteins that promote mRNA degradation, inhibit mRNA translation or inhibit viral polymerases. Some examples for immunomodulatory functions of type I IFNs are positive as well as negative effects on maturation and function of DCs, type 1 Th cell responses, and macrophage activity (Bartholomé et al., 1999; Bouchonnet et al., 2002; Gabriele et al., 2004; Hermann et al., 1998; Hibbert et al., 2003; López-Collazo et al., 1998; Luft et al., 1998). Furthermore, type I IFNs have been shown to dampen the recruitment of neutrophils by suppressing the production of CXCL1 and CXCL2 in epithelial cells during virus infections (Seo et al., 2011; Stock et al., 2014; Uyangaa et al., 2015). However, this is not mirrored in the setting studied herein, where a lack of mast cells (and therefore a source of IFN- $\beta$ ) was accompanied by a decrease in CXCL1 in the inflamed tissue and no change in neutrophil number could be observed during the resolution of the inflammation. Another function of IFN- $\beta$ that has been described is the induction of IL-10 production in macrophages (Chang et al., 2007). While there was no autocrine effect on the release of IL-10 from mast cells observable in vitro in this thesis, IFN- $\beta$ might influence the release of IL-10 in neighbouring M2 macrophages in the tissue.

An effect of IFN- $\alpha / \beta$ on phagocytosis by macrophages has already been demonstrated 36 years ago (Rollag et al., 1984). Rollag et al. (1984) found that both attachment and ingestion of bacteria or erythrocytes by macrophages were enhanced by treatment with IFN$\alpha / \beta$, indicating for alterations of the surface receptors. This again fits to the study of Jachetti et al. (2019) who demonstrated an enhancing effect of mast cells on the efferocytic activity of macrophages accompanied by an increased expression of their phagocytic receptor Tim4. Finally, the enhancing effect of macrophage-derived IFN- $\beta$ on efferocytosis by macrophages and therefore its role in the promotion of the resolution of inflammation has been demonstrated in vivo recently (Kumaran Satyanarayanan et al., 2019). Here, we observed a decrease in phagocytic and efferocytic activity of macrophages in the absence of mast cells and present mast cells as a novel source for non-viral TLR-induced IFN- $\beta$. Other mast cellderived mediators besides IFN- $\beta$, namely IL-1 $\beta$, IL-4, IL-13, and $P G E_{2}$, have been demonstrated to increase the phagocytic activity of macrophages as well (Berry et al., 2007; Daseke et al., 2020; Korns et al., 2011; Schenk et al., 2014; Szanto et al., 2010; 
Wainszelbaum et al., 2006; Welch et al., 2003). Therefore, one of these mediators or a combination of them could be responsible for the enhancing effect of mast cells on macrophage phagocytosis and efferocytosis (Figure 36). To verify the effect of mast cells and their mediators in vivo, models with mast cells selectively deficient for these mediators would be useful in future studies.

In this study we add evidence to the role of mast cells in regulating the ability of macrophages to phagocytose pathogens and efferocytose apoptotic cells, thereby contributing to the resolution of inflammation. Furthermore, we present mast cells as a novel source for IFN- $\beta$ induced by non-viral TLR signaling from endolysosomal compartments. Taken together, this underlines the plasticity of mast cells, ranging from inducers and promoters of inflammation to supporters of the resolution of inflammation. The better understanding of the variability of mast cells in dependence of their biological settings, regarding their environment as wells as the phase of the inflammation, can help to regulate mast cells in pathological conditions. Furthermore, the understanding of the complex interactions of immune cells during inflammation can help to find new ways treating diseases associated with chronic inflammation. 


\section{Zusammenfassung}

Mastzellen sind langlebige Leukozyten, die in beinahe allen vaskularisierten Geweben vorkommen, besonders gehäuft jedoch an Grenzflächen zur äußeren Umgebung wie Schleimhäuten und der Haut. Durch diese Position gehören sie zu den ersten Immunzellen, die in Kontakt mit schädlichen Substanzen aus der Umwelt, wie Mikroben, Viren, oder toxischen Substanzen, kommen. Das charakteristischste Merkmal von Mastzellen sind ihre Granula, welche mit einer großen Menge verschiedener Mediatoren gefült sind, die sofort nach Aktivierung der Zelle freigesetzt werden können.

Mastzellen sind hauptsächlich als pro-inflammatorische Zellen bekannt, vor allem in ihrer Rolle bei IgE-vermittelten allergischen Reaktionen. Vermehrt weisen Studien jedoch auch auf potentielle anti-inflammatorische Funktionen von Mastzellen hin (Galli et al., 2008; Kraneveld et al., 2012), die meist dem Zytokin IL-10 zugeordnet werden konnten (Chan et al., 2013; Depinay et al., 2006; Grimbaldeston et al., 2007). Während früher hauptsächlich verschiedene Kit-abhängige Mastzell-defiziente Mausmodelle verwendet wurden, die neben der Mastzell-Defizienz auch weitere Veränderungen des Immunsystems aufweisen, werden inzwischen meist Kit-unabhängige Mastzell-defiziente Mausmodelle verwendet. Untersuchungen zeigten, dass Studien mit Kit-abhängigen und Kit-unabhängigen Mauslinien häufig zu unterschiedlichen Ergebnissen führen (Rodewald \& Feyerabend, 2012). Um die Hinweise auf anti-inflammatorische, entzündungsauflösende Eigenschaften der Mastzellen zu bestätigen, sind deshalb weitere Studien mit Kit-unabhängigen Mastzell-defizienten Mausmodellen notwendig. Ziel dieser Arbeit war es daher, die Rolle von Mastzellen während der Entzündungsauflösung unter Verwendung einer Kit-unabhängigen Mastzell-defizienten Mauslinie zu untersuchen.

Um zunächst ein geeignetes Modell einer lokalen Entzündung in der Maus zu identifizieren, wurden dafür die beiden am häufigsten verwendeten Modelle lokal-induzierter Entzündungen miteinander verglichen, nämlich Lipopolysaccharid (LPS)- und Zymosan-induzierte Entzündungen. Sowohl bei LPS als auch bei Zymosan handelt es sich um Liganden von Tolllike-Rezeptoren (TLR), die eine große Rolle bei der Erkennung von Pathogenen im Rahmen der angeborenen Immunität spielen (Mogensen, 2009). Während bei der LPS-induzierten Entzündung systemische Auswirkungen auf die Konzentration von Zytokinen im Blut sowie auf das Lipidom in unbehandeltem Gewebe festgestellt wurden, war dies bei der Zymosaninduzierten Entzündung nicht der Fall (Hahnefeld \& Kornstädt et al., 2021). Zudem war die lokale Reaktion in Hinsicht auf die Rekrutierung von Immunzellen und die Größe des Ödems der behandelten Pfote im Zymosan-Modell deutlich ausgeprägter als im LPS-Modell. Somit 
wurde das Zymosan-Modell für die Untersuchung der Rolle von Mastzellen in der Auflösung von lokalen Entzündungen gewählt.

Um einen Überblick über die Umgebung von Mastzellen während einer lokalen Entzündung zu gewinnen, wurde im nächsten Schritt Gewebe mittels Multi-Epitop-Liganden-Kartographie (MELK) untersucht. Bei MELK handelt es sich um eine automatisierte ImmunfluoreszenzTechnologie, die die gleichzeitige Analyse einer großen Anzahl Proteine in einem Gewebeschnitt ermöglicht. Durch maschinelles Lernen unter Verwendung der Software CellProfiler und histoCAT können im Anschluss verschiedene Zelltypen im Gewebe identifiziert und ihre Nachbarschaftsbeziehungen zueinander analysiert werden.

Zunächst wurden verschiedene Phasen des Entzündungsverlaufs durch die Intensität der Zymosan-induzierten mechanischen Hypersensitivität identifiziert. Die zellulären Vorgänge zu diesen Zeitpunkten wurden anschließend mittels MELK Analyse untersucht. Während im unbehandelten Gewebe keinerlei Neutrophile identifiziert werden konnte, war eine große Anzahl Neutrophile im Bereich des Zymosan-gefüllten Kerns der Entzündung und darum herum während der akuten Entzündung, 4 Stunden nach Zymosan-Injektion bei ansteigender mechanischen Hypersensitivität, zu finden. Während der Entzündungsauflösung, 48 Stunden nach Zymosan-Injektion bei abnehmender mechanischer Hypersensitivität, konnte eine stärker auf das Zymosan-Feld begrenzte NeutrophilenPopulation und eine zunehmende Anzahl an Makrophagen beobachtet werden. Auch eine vermehrte Efferozytose von Neutrophilen durch Makrophagen konnte während der Entzündungsauflösung festgestellt werden. Die Einwanderung von Neutrophilen als primär phagozytierende Zellen, gefolgt von der Einwanderung von Makrophagen, zu deren Aufgabe die Beseitigung von apoptotischen Zellen gehört, entspricht dem klassischen Verlauf einer akuten Entzündung. Zudem konnte eine vermehrte Polarisation der Makrophagen in eine pro- und eine anti-inflammatorische Population beobachtet werden. Pro-inflammatorische Makrophagen befanden sich im Zymosan-gefüllten Kern der Entzündung, antiinflammatorische Makrophagen im daran angrenzenden Bereich. Die Nachbarschaftsanalyse von Mastzellen ergab, dass diese anti-inflammatorischen Makrophagen zum Zeitpunkt der Entzündungsauflösung häufiger als andere Zelltypen in direkter Nachbarschaft zu Mastzellen liegen.

Um den Einfluss von Mastzellen auf den Verlauf der Entzündung zu untersuchen, wurde in einem nächsten Schritt Gewebe von Mastzell-defizienten Mcpt5-DTA Cre ${ }^{+}$Mäusen mit Mcpt5-DTA Cre- Mäusen ohne Mastzell-Defizienz mittels MELK verglichen. In Mcpt5-DTA $\mathrm{Cre}^{+}$Mäusen führt die Expression der Cre Rekombinase, die sich unter der Kontrolle des Mastzellprotease 5 Promotors befindet, zur Aktivierung der Expression von katalytisch aktivem Diphtheria Toxin A, was wiederum zum Zelltod von Mastzellen führt (Dudeck et al., 
2011). Interessanterweise konnte in den Mastzell-defizienten Mäusen ein Hinweis auf verringerte Efferozytose von Neutrophilen durch Makrophagen entdeckt werden. Dieser Effekt, sowie eine Veränderung der Phagozytose Aktivität von Makrophagen, konnte bereits in einer vorherigen Studie gezeigt werden, jedoch unter Verwendung von C57BL/6 Kit ${ }^{\mathrm{W} \text {-sh }}$ Mäusen, die neben der Mastzell-Defizienz auch weitere Veränderungen des Immunsystems aufweisen (Jachetti et al., 2019).

Um den Einfluss von Mastzellen auf die Phagozytose und Efferozytose Aktivität von Makrophagen weiter zu untersuchen, wurde in einem nächsten Schritt pH-sensitives pHrodoZymosan in Pfoten von Mcpt5-DTA Cre Mäuse injiziert, welches im sauren Milieu von Lysosomen durch Fluoreszenz detektiert werden kann. Mittels multiparametrischer Durchflusszytometrie konnte ebenfalls eine Verringerung der Efferozytose von Neutrophilen durch Makrophagen, sowie eine Verringerung der Phagozytose von Zymosan durch Makrophagen detektiert werden. Unterschiede in der Phagozytose Aktivität von Neutrophilen oder Veränderungen der Anzahl verschiedener Immunzellen oder Makrophagen Phänotypen konnten währenddessen nicht festgestellt werden. Somit konnte der Einfluss von Mastzellen auf die Phagozytose und Efferozytose Aktivität von Makrophagen bestätigt werden.

Um ungerichtet Mediatoren zu identifizieren, die von Mastzellen nach Zymosan-Induktion freigesetzt werden und möglicherweise die Phagozytose Aktivität von Makrophagen beeinflussen, wurde eine mRNA Sequenzierung mit Mastzellen durchgeführt. Bei den hierfür verwendeten Zellen handelt es sich um Mastzellen die aus dem Knochenmark unter Einfluss von Wachstumsfaktoren ausdifferenziert wurden und anschließend per Durchflusszytometrie aufgereinigt wurden. Die Analyse der Sequenzierungdaten ergab, dass die Typ I Interferon Antwort nach Zymosan-Behandlung gegenüber unbehandelten Zellen stark hochreguliert ist. Typ I Interferone sind hauptsächlich im Zusammenhang mit viralen Infektionen bekannt. Zuerst beschrieben wurden sie bereits 1957 (Isaacs \& Lindenmann, 1957), aktuell ist vor allem ihre zentrale Rolle bei der Immunantwort auf SARS-CoV-2 von großem Interesse (Bastard et al., 2020; Zhang et al., 2020). Neben Funktionen bei viralen Infektionen sind inzwischen auch Funktionen von Typ I Interferonen in bakteriellen Infektionen, sowie bei Pilzinfektionen und Parasitenbefall beschrieben (McNab et al., 2015).

Zur Validierung der Sequenzierungsergebnisse wurde die Freisetzung der Typ I Interferone IFN- $\alpha$ und IFN- $\beta$ durch Mastzellen auf Proteinebene untersucht. IFN- $\alpha$ konnte nicht detektiert werden, die Freisetzung von IFN- $\beta$ konnte jedoch mittels ELISA sowohl nach Induktion der Mastzellen durch Zymosan, als auch durch LPS bestätigt werden. Während die Produktion von Typ I Interferonen von Mastzellen als Antwort auf intrazelluläre TLR Induktion, wie beispielsweise durch Viren, bekannt ist (Sandig \& Bulfone-Paus, 2012a), galt die Produktion 
von IFN- $\beta$ nach Induktion von extrazellulären TLR in Mastzellen bisher als ausgeschlossen (Dietrich, Rohde, et al., 2010; Keck et al., 2011).

Es ist bekannt, dass die Typ I Interferon Antwort erst nach Internalisierung der extrazellulären TLR stattfinden kann, da dadurch die Rekrutierung von und Interaktion mit TRAF3 ermöglicht wird, was wiederum Voraussetzung für die Aktivierung der Transkription von Typ I Interferonen ist (Bauernfeind \& Hornung, 2009; Kagan et al., 2008; Perkins \& Vogel, 2015; Tanimura et al., 2008). Eine der Studien begründete die fehlende Induktion der Typ I Interferon Antwort in Mastzellen auf den TLR4 Agonisten LPS damit, dass Mastzellen den Rezeptor-Pathogen Komplex nicht aktiv internalisieren können. Dies ist jedoch im Widerspruch mit mehreren Studien, die die Internalisierung des TLR4 Rezeptors in Mastzellen zeigen konnten (Meng et al., 2013; Okumura et al., 2003; Pérez-Rodríguez et al., 2020). In Übereinstimmung damit konnte die Freisetzung von IFN- $\beta$ sowohl nach Zymosanals auch nach LPS- Induktion durch pharmakologische Inhibierung der RezeptorInternalisierung sowie der Endosom-Reifung in Mastzellen blockiert werden, während das pro-inflammatorisch Zytokin IL-1ß, das von nicht-internalisierten TLR induziert wird, weiter freigesetzt wurde. Im Rahmen dieser Arbeit konnte somit gezeigt werden, dass Mastzellen entgegen bisheriger Erkenntnisse dazu in der Lage sind, IFN- $\beta$ nach Induktion von primär membranständigen TLR freizusetzen, und dass die Typ I Interferon Antwort darüber hinaus sogar die dominante Reaktion von Mastzellen auf Zymosan-Induktion darstellt (Kornstädt et al., 2021).

Interessanterweise wurde der Effekt von IFN- $\beta$ auf die Efferozytose Aktivität von Makrophagen und daraus folgend der entzündungsauflösende Effekt dieses Zytokins erst kürzlich gezeigt (Kumaran Satyanarayanan et al., 2019). Durch die Analyse von ZytokinLeveln im Gewebe von Mastzell-defizienten Mcpt5-DTA Cre ${ }^{+}$und Kontroll Mcpt5-DTA CreMäusen konnte außerdem eine Verringerung der Konzentration von IL-4 und CXCL1 in Abwesenheit von Mastzellen festgestellt werden. CXCL1 ist hauptsächlich für die Rekrutierung von Neutrophilen bekannt. Obwohl eine frühere Studie zeigen konnte, dass Mastzellen in die Rekrutierung von Neutrophilen mittels CXCL1 während der frühen Phase einer Entzündung involviert sind, konnte keine veränderte Anzahl von Neutrophilen während der Entzündungsauflösung festgestellt werden. Die Rolle von CXCL1 während dieser späten Entzündungsphase scheint also nicht die Rekrutierung von Neutrophilen zu beinhalten. Andere bekannte Funktionen von CXCL1, die zur Auflösung der Entzündung passend erscheinen, sind die Förderung von Angiogenese und Epithelisierung im Rahmen der Wundheilung (Castela et al., 2017).

IL-4 ist hauptsächlich für den Einfluss auf die Polarisierung von Makrophagen in Richtung eines anti-inflammatorischen Phänotyps und verstärkter CD206 Expression bekannt, doch 
ein solcher Effekt konnte durch multiparametrische Durchflusszytometrie nicht beobachtet werden. Darüber hinaus wurde auch schon demonstriert, dass IL-4 die Fähigkeit von Makrophagen zur Phagozytose und Efferozytose von Neutrophilen erhöhen kann (Daseke et al., 2020; Wainszelbaum et al., 2006). Ein verstärkender Einfluss auf die Phagozytose durch Makrophagen wurde außerdem bereits für IL-1 $\beta$, IL-13 und PGE 2 gezeigt, (Korns et al., 2011; Schenk et al., 2014; Wainszelbaum et al., 2006) welche auch nach Zymosan und LPS von Mastzellen freigesetzt wurden. Der Effekt von Mastzellen auf die Phagozytose und Efferozytose Aktivität von Makrophagen der in dieser Arbeit beobachtet wurde, kann dementsprechend durch einen dieser Mediatoren, oder einer Kombination der Mediatoren erzeugt werden. Genauere Untersuchungen hierzu in zukünftigen Studien erfordern beispielsweise Mausmodelle, in denen diese Mediatoren selektiv in Mastzellen depletiert sind. Durch ihren verstärkenden Einfluss auf die Phagozytose und Efferozytose haben Mastzellen somit das Potential, die Auflösung von Entzündungen zu beschleunigen.

Die Position von Mastzellen im entzündeten Gewebe innerhalb des anti-inflammatorischen Bereichs, die in dieser Arbeit mittels MELK untersucht wurde, deutete zudem auch auf ein anti-inflammatorisches, entzündungsauflösendes Potential von Mastzellen während der späten Phase einer lokalen Entzündung hin. Neben IL-4, IL-13, CXCL1 und PGE 2 konnte auch die Freisetzung von IL-10 von Mastzellen nach LPS- und Zymosan-Behandlung festgestellt werden, welches ebenfalls für anti-inflammatorische Eigenschaften bekannt ist (Ip et al., 2017; Sabat et al., 2010)

Insgesamt konnte im Rahmen dieser Arbeit die Position der Mastzellen im einem antiinflammatorischen Bereich des Gewebes, die Produktion diverser Mediatoren mit antiinflammatorischen, entzündungsauflösenden Eigenschaften (IFN- $\beta$, IL-4, IL-10, IL-13, CXCL1, PGE 2 ), sowie ihr verstärkender Einfluss auf die Phagozytose und Efferozytose Aktivität von Makrophagen gezeigt werden. Zusammengenommen lässt dies auf einen entzündungauflösenden Phänotyp während der späten Phase von lokalen Entzündungen schließen. Dies spricht für einen wechselnder Phänotyp von Mastzellen je nach Umgebung oder auch Phase der Entzündung, wie bereits in vorhergehenden Studien beschrieben (Galli et al., 2008, 2011). 


\section{List of abbreviations}

\section{A}

ALR - AIM2-like receptor

ANOVA - Analysis of variance

APC $\cdot$ Allophycocyanin

B

BCA · Bicinchoninic acid

BMDM - Bone marrow-derived macrophage

BMMC - Bone marrow-derived mast cell

BSA · Bovine serum albumin

\section{C}

CLR $\cdot$ C-type lectin receptor

cDNA - complementary DNA

CMP · Common myeloid progenitor

COX $\cdot$ Cyclooxygenase

Cre $\cdot$ Causes recombination

CTMC · Connective tissue mast cells

D

DAMP · danger-associated molecular pattern

DAPI - 4', 6-Diamidin-2-phenylindol

$\mathrm{DE} \cdot$ differentially expressed

DMEM - Dulbecco's Modified Eagle Medium

DMSO - Dimethyl sulfoxide

DNA - deoxyribonucleic acid

dsDNA - double stranded DNA

DTA - diphtheria toxin $A$

E

EETs · epoxy-eicosatrienoic acids

EGF · epidermal growth factor
ELISA · enzyme-linked immunoassay

ERK · extracellular signal-regulated kinases

$\mathbf{F}$

FACS - Fluorescence-activated cell scanning

FBS - Fetal bovine serum

FC · flow cytometry

FGF · fibroblast growth factor

FSC-A · forward scatter area

FSC-H · forward scatter height

FSC-W · forward scatter width

FceRI · High-affinity lgE receptor

G

G-CSF · granulocyte colony-stimulating factor

GM-CSF · granulocyte-macrophage colony-stimulating factor

$\mathbf{H}$

$\mathrm{H}_{2} \mathrm{O}_{2} \cdot$ Hydrogen peroxide

HEPES · 4-(2-hydroxyethyl)-1piperazineethanesulfonic acid

HETEs · hydroxyeicosatetraenoic acids

HPETEs · hydroperoxy-leicosatetraenoic acids

I

I $\mathrm{B} \cdot$ inhibitor of NF-KB

IFITM - interferon-induced transmembrane

IFN $\cdot$ interferon

IFNAR $\cdot$ interferon- $\alpha / \beta$ receptor

IFNGR · interferon gamma receptor

IFNLR · interferon lambda receptor

$\mathrm{lg} \cdot$ immunoglobulin 
IKK $\cdot$ IKB kinase

$\mathrm{IL} \cdot$ Interleukin

iNOS · inducible NOS

IRAK · interleukin-1 receptor associated kinase

IRF · interferon regulatory factor

ISG · interferon stimulated genes

ISRE - interferon-stimulated regulatory element

J

JAK-STAT · Janus kinase-signal transducer and activator of transcription

L

LC-MS/MS · Liquid chromatographytandem mass spectrometry

LOX · lipoxygenase

loxP · Locus of X-over P1

LPS · Lipopolysaccharide

LRR · leucine-rich repeat

LTs · leukotriens

LXs · lipoxins

LysoPC · Lysophosphatidylcholine

M

MAL $\cdot$ MyD88-adaptor-like

MAPK - mitogen-activated protein kinase

$\mathrm{MC}_{\mathrm{C}} \cdot$ chymse-only mast cell

$\mathrm{MCp} \cdot$ mast cell progenitor

MCP-1 - monocyte chemoattractant protein-1

Mcpt $\cdot$ mast cell protease

M-CSF - Macrophage colony stimulation factor

$\mathrm{MC}_{\mathrm{T}} \cdot$ tryptase-only mast cell

$\mathrm{MC}_{\mathrm{TC}} \cdot$ tryptase- and chymase-positive mast cell
MEK - mitogen-activated proteinkinase kinase

MELC · multi-epitope ligand cartography

MHC - major histocompatibility complex

MMC $\cdot$ mucosal mast cells

mRNA - messenger RNA

MW - Molecular weight

$\mathrm{Mx} \cdot$ Myxovirus resistance

MyD88 - Myeloid differentiation primaryresponse protein 88

$\mathbf{N}$

NF-kB - nuclear factor kappa $B$

NGF - Nerve growth factor

NGF · nerve growth factor

NLR · Nod-like receptor

$\mathrm{NO} \cdot$ Nitric oxide

NOS - Nitric oxide synthases

NSAIDs · non-steroidal anti-inflammatory drugs

0

OAS · oligoadenylate synthase

$\mathbf{P}$

PAFR - platelet-activating factor receptor

PAMP · pathogen-associated molecular pattern

PBS - Phosphate-buffered saline

PCR - Polymerase chain reaction

PDGF · platelet-derived growth factor

$\mathrm{PE} \cdot$ phycroerythrin

PFA - Paraformaldehyde

PGs · prostaglandins

$\mathrm{PI}$ propidion iodide

PMN · polymorphnucelar neutrophil

PRR - pattern recognition receptors, pattern recognition receptor

PUFA · polyunsaturated fatty acid 
PWL · paw withdrawal latency

\section{$\mathbf{Q}$}

$\mathrm{QC} \cdot$ quality control

qPCR - quantitative realtime PCR

\section{$\mathbf{R}$}

$\mathrm{RLR} \cdot$ RIG-I-like receptor

ROS - reactive oxygen species

RNA - ribonucleic acid

\section{$\mathbf{S}$}

S.E.M. - Standard error of the mean

S1P . Sphingosine-1-phosphate

SARM - Sterile $\alpha$ - and armadillo-motifcontaining protein

SCF $\cdot$ stem-cell factor

SE $\cdot$ steryl esthers

$\mathrm{SH} 2 \cdot \mathrm{SRC}$ homology 2

SSC-A $\cdot$ sidescatter area
SSC-H $\cdot$ sidescatter height

SSC-W $\cdot$ sidescatter width

$\mathbf{T}$

TGF $\beta \cdot$ transforming growth factor beta

TIR · Toll-interleukin-1 receptor

TIRAP . TIR domain-containing adaptor protein

TLR $\cdot$ Toll-like receptor

TNF- $\alpha \cdot$ tumor necrosis factor alpha

TRAM - TRIF related adaptor molecule

TRIF . TIR domain containing adaptor protein inducing IFN- $\beta$

TRIM - tripartite motif family

Tris · Tris(hydroxymethyl)aminomethane

$\mathbf{U}$

USP · ubiquitin specific peptidase

V

VEGF - vascular endothelial growth factor 


\section{Table of figures}

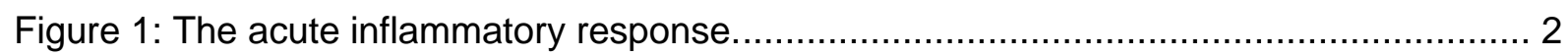

Figure 2: Anti-inflammatory versus pro-resolution treatment of inflammation....................... 3

Figure 3: Extracellular and intracellular TLR2 and TLR4 signaling...............................13

Figure 4: Downstream signaling cascade of IFNs.........................................................

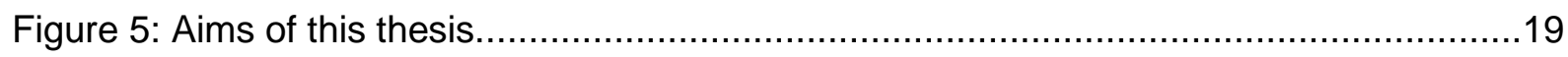

Figure 6: Gating strategy for identification of immune cells in the inflamed paw of mice 5

$\mathrm{h}$ after intraplantar injection of zymosan or LPS.....................................30

Figure 7: Gating strategy for immune cells and their subtypes in paws of Mcpt5-DTA Cre- and Cre+ mice $48 \mathrm{~h}$ after injection of zymosan $(10 \mu \mathrm{l}, 12 \mathrm{mg} / \mathrm{ml})$.

Figure 8: Gating strategy for FACS sorting of mature BMMCs for RNA sequencing. ............39

Figure 9: Comparison of mechanical hypersensitivity induced by LPS and zymosan ..........45

Figure 10: Edema formation and immune cell recruitment induced by LPS and Zymosan....47

Figure 11: Distinct lipid profiles and chemokine levels after injection of zymosan or LPS....49

Figure 12: Mast cells are located in the anti-inflammatory region during the resolution phase, surrounding the zymosan core of the inflammation.

Figure 13: Identification and characterization of immune cell phenotypes in MELC images of inflamed paws.

Figure 14: Efferocytosis increases during resolution of inflammation. .54

Figure 15: Mast cells are located in anti-inflammatory neighbourhood during resolution of zymosan-induced inflammation. .55

Figure 16: Mast cells are decreased in paws of Mcpt5-DTA Cre+ mice compared to Cremice. .56

Figure 17: M1 macrophage phagocytosis of neutrophils is reduced in mast cell-deficient Mcpt5-DTA Cre+ mice compared to Cre- control mice.

Figure 18: Decreased Ly6G signal in M1 macrophages in paws of mast cell-deficient Mcpt5-DTA Cre+ mice. .58

Figure 19: Mast cells influence phagocytosing activity of macrophages during resolution phase of zymosan-induced inflammation. 60 
Figure 20: IL-4 and CXCL1 are decreased in mast cell-deficient mice during inflammation.

Figure 21: Levels of prostanoids during local inflammation in Mcpt5-DTA Cre mice .63

Figure 22: Flow cytometry analysis shows high purity of BMMCs matured according to standard protocol.

Figure 23: Timecourse of release of IL-10 and IL-1 $\beta$ by mast cells after induction with zymosan or LPS

Figure 24: Chemokines and prostanoids released by mast cells after induction with zymosan or LPS. .66

Figure 25: No release of IL-10 and IL-1 $\beta$ in response to IL-13, S1P or LysoPC. (A) .68

Figure 26: Release of IL-10 does not depend on IgE-mediated degranulation but on COX signaling .69

Figure 27: IL-10 release by BMMCs used for RNA sequencing. .70

Figure 28: Differential expression analysis shows that most genes are commonly regulated after treatment for $24 \mathrm{~h}$ or $48 \mathrm{~h}$ compared to untreated samples....73

Figure 29: Gene ontology term analysis reveals type I IFN response after induction of BMMCs with zymosan. .75

Figure 30: Highly upregulated genes are mostly interferon-inducible. .76

Figure 31: IFN- $\beta$ is produced by mast cells in vitro and in vivo. 78

Figure 32: IFN- $\beta$ signaling is not required for release of IL-10 in mast cells. .79

Figure 33: Release of IFN- $\beta$ does not require ERK, NOD2 or PAFR. .81

Figure 34: Release of IFN- $\beta$ by mast cells requires receptor internalization and endosome maturation.

Figure 35: Mast cells are located in anti-inflammatory neighbourhood and influence phagocytosis and efferocytosis activity of macrophages during resolution of inflammation.

Figure 36: Mast cells release a plethora of mediators in respose to TLR activation, of which some have been shown to influence the phagocytosis and efferocytosis activity of macrophages. .90 


\section{References}

Acoba, M. G., Senoo, N., \& Claypool, S. M. (2020). Phospholipid ebb and flow makes mitochondria go. In Journal of Cell Biology. https://doi.org/10.1083/JCB.202003131

Aiyar, N., Disa, J., Ao, Z., Ju, H., Nerurkar, S., Willette, R. N., Macphee, C. H., Johns, D. G., \& Douglas, S. A. (2007). Lysophosphatidylcholine induces inflammatory activation of human coronary artery smooth muscle cells. Molecular and Cellular Biochemistry, 295(1-2), 113-120. https://doi.org/10.1007/s11010-006-9280-x

Ajiro, Y., Saegusa, N., Giles, W. R., Stafforini, D. M., \& Spitzer, K. W. (2011). Plateletactivating factor stimulates sodium-hydrogen exchange in ventricular myocytes. American Journal of Physiology - Heart and Circulatory Physiology. https://doi.org/10.1152/ajpheart.00607.2011

Akira, S., \& Takeda, K. (2004). Toll-like receptor signalling. Nature Reviews Immunology, 4(7), 499-511. https://doi.org/10.1038/nri1391

Akira, S., Uematsu, S., \& Takeuchi, O. (2006). Pathogen recognition and innate immunity. In Cell (Vol. 124, Issue 4, pp. 783-801). Cell. https://doi.org/10.1016/j.cell.2006.02.015

Albert, C. J., Crowley, J. R., Hsu, F. F., Thukkani, A. K., \& Ford, D. A. (2001). Reactive chlorinating species produced by myeloperoxidase target the vinyl ether bond of plasmalogens: Identification of 2-chlorohexadecanal. Journal of Biological Chemistry. https://doi.org/10.1074/jbc.M101447200

Allavena, P., Chieppa, M., Monti, P., \& Piemonti, L. (2004). From pattern recognition receptor to regulator of homeostasis: The double-faced macrophaqe mannose receptor. In Critical Reviews in Immunology. https://doi.org/10.1615/CritRevImmunol.v24.i3.20

Alvarez, Y., Municio, C., Alonso, S., Sánchez Crespo, M., \& Fernández, N. (2009). The Induction of IL-10 by Zymosan in Dendritic Cells Depends on CREB Activation by the Coactivators CREB-Binding Protein and TORC2 and Autocrine PGE 2 . The Journal of Immunology. https://doi.org/10.4049/jimmunol.0900312

Amir, E. D., Davis, K. L., Tadmor, M. D., Simonds, E. F., Levine, J. H., Bendall, S. C., Shenfeld, D. K., Krishnaswamy, S., Nolan, G. P., \& Pe'er, D. (2013). viSNE enables visualization of high dimensional single-cell data and reveals phenotypic heterogeneity of leukemia. Nature Biotechnology, 31(6), 545-552. https://doi.org/10.1038/nbt.2594

Ashina, K., Tsubosaka, Y., Nakamura, T., Omori, K., Kobayashi, K., Hori, M., Ozaki, H., \& Murata, T. (2015). Histamine induces vascular hyperpermeability by increasing blood flow and endothelial barrier disruption in vivo. PLOS ONE. https://doi.org/10.1371/journal.pone.0132367

Barbalat, R., Lau, L., Locksley, R. M., \& Barton, G. M. (2009). Toll-like receptor 2 on inflammatory monocytes induces type I interferon in response to viral but not bacterial ligands. Nature Immunology, 10(11), 1200-1207. https://doi.org/10.1038/ni.1792. PM 19801985

Bartholomé, E. J., Willems, F., Crusiaux, A., Thielemans, K., Schandene, L., \& Goldman, M. (1999). IFN- $\beta$ interferes with the differentiation of dendritic cells from peripheral blood mononuclear cells: Selective inhibition of CD40-dependent interleukin-12 secretion. Journal of Interferon and Cytokine Research. https://doi.org/10.1089/107999099313910

Bastard, P., Rosen, L. B., Zhang, Q., Michailidis, E., Hoffmann, H. H., Zhang, Y., Dorgham, K., Philippot, Q., Rosain, J., Béziat, V., Manry, J., Shaw, E., Haljasmägi, L., Peterson, P., Lorenzo, L., Bizien, L., Trouillet-Assant, S., Dobbs, K., de Jesus, A. A., ... Casanova, J. L. (2020). Autoantibodies against type I IFNs in patients with life-threatening COVID19. Science (New York, N.Y.). https://doi.org/10.1126/science.abd4585

Bauernfeind, F., \& Hornung, V. (2009). TLR2 joins the interferon gang. Nature Immunology, 10(11), 1139-1141. https://doi.org/10.1038/ni1109-1139

Bayani, A., Dunster, J. L., Crofts, J. J., \& Nelson, M. R. (2020). Spatial considerations in the resolution of inflammation: Elucidating leukocyte interactions via an experimentallycalibrated agent-based model. PLoS Computational Biology. https://doi.org/10.1371/journal.pcbi.1008413

Beck-Schimmer, B., Schwendener, R., Pasch, T., Reyes, L., Booy, C., \& Schimmer, R. C. 
(2005). Alveolar macrophages regulate neutrophil recruitment in endotoxin-induced lung injury. Respiratory Research. https://doi.org/10.1186/1465-9921-6-61

Benezeder, T., \& Wolf, P. (2019). Resolution of plaque-type psoriasis: what is left behind (and reinitiates the disease). In Seminars in Immunopathology.

https://doi.org/10.1007/s00281-019-00766-z

Bennett, J. M., Reeves, G., Billman, G. E., \& Sturmberg, J. P. (2018). Inflammation-nature's way to efficiently respond to all types of challenges: Implications for understanding and managing "the epidemic" of chronic diseases. Frontiers in Medicine. https://doi.org/10.3389/fmed.2018.00316

Benoit, M., Desnues, B., \& Mege, J.-L. (2008). Macrophage Polarization in Bacterial Infections. The Journal of Immunology. https://doi.org/10.4049/jimmunol.181.6.3733

Benveniste, E. N., \& Qin, H. (2007). Type I interferons as anti-inflammatory mediators. Science's STKE : Signal Transduction Knowledge Environment. https://doi.org/10.1126/stke.4162007pe70

Bernotiene, E., Palmer, G., Talabot-Ayer, D., Szalay-Quinodoz, I., Aubert, M. L., \& Gabay, C. (2004). Delayed resolution of acute inflammation during zymosan-induced arthritis in leptin-deficient mice. Arthritis Research \& Therapy. https://doi.org/10.1186/ar1174

Berrozpe, G., Agosti, V., Tucker, C., Blanpain, C., Manova, K., \& Besmer, P. (2006). A Distant Upstream Locus Control Region Is Critical for Expression of the Kit Receptor Gene in Mast Cells. Molecular and Cellular Biology. https://doi.org/10.1128/mcb.0185405

Berry, A., Balard, P., Coste, A., Olagnier, D., Lagane, C., Authier, H., Benoit-Vical, F., Lepert, J. C., Séguéla, J. P., Magnaval, J. F., Chambon, P., Metzger, D., Desvergne, B., Wahli, W., Auwerx, J., \& Pipy, B. (2007). IL-13 induces expression of CD36 in human monocytes through PPARy activation. European Journal of Immunology. https://doi.org/10.1002/eji.200636625

Biedermann, T., Kneilling, M., Mailhammer, R., Maier, K., Sander, C. A., Kollias, G., Kunkel, S. L., Hültner, L., \& Röcken, M. (2000). Mast cells control neutrophil recruitment during T cell-mediated delayed-type hypersensitivity reactions through tumor necrosis factor and macrophage inflammatory protein 2. Journal of Experimental Medicine. https://doi.org/10.1084/jem.192.10.1441

Bogdan, C., Mattner, J., \& Schleicher, U. (2004). The role of type I interferons in non-viral infections. In Immunological Reviews. https://doi.org/10.1111/j.0105-2896.2004.00207.x

Boldeanu, M. V., Siloşi, I., Ghiluşi, M., Cojocaru, M., Biciuşcă, V., Avrămescu, C. S., Cojocaru, I. M., Ciurea, T., Albu, D. F., \& Siloşi, C. A. (2014). Investigation of inflammatory activity in ulcerative colitis. Romanian Journal of Morphology and Embryology.

Borden, E. C., Sen, G. C., Uze, G., Silverman, R. H., Ransohoff, R. M., Foster, G. R., \& Stark, G. R. (2007). Interferons at age 50: past, current and future impact on biomedicine. Nature Reviews Drug Discovery, 6(12), 975-990. https://doi.org/10.1038/nrd2422

Bot, I., Shi, G. P., \& Kovanen, P. T. (2015). Mast cells as effectors in atherosclerosis. Arteriosclerosis, Thrombosis, and Vascular Biology. https://doi.org/10.1161/ATVBAHA.114.303570

Botos, I., Segal, D. M., \& Davies, D. R. (2011). The structural biology of Toll-like receptors. In Structure. https://doi.org/10.1016/j.str.2011.02.004

Bouchonnet, F., Boechat, N., Bonay, M., \& Hance, A. J. (2002). Alpha/beta interferon impairs the ability of human macrophages to control growth of Mycobacterium bovis BCG. Infection and Immunity. https://doi.org/10.1128/IAI.70.6.3020-3025.2002

Boxx, G. M., \& Cheng, G. (2016). The Roles of Type i Interferon in Bacterial Infection. In Cell Host and Microbe. https://doi.org/10.1016/j.chom.2016.05.016

Boyce, J. A. (2007). Mast cells and eicosanoid mediators: A system of reciprocal paracrine and autocrine regulation. In Immunological Reviews. https://doi.org/10.1111/j.1600065X.2007.00512.x

Boys, I. N., Xu, E., Mar, K. B., De La Cruz-Rivera, P. C., Eitson, J. L., Moon, B., \& Schoggins, J. W. (2020). RTP4 Is a Potent IFN-Inducible Anti-flavivirus Effector 
Engaged in a Host-Virus Arms Race in Bats and Other Mammals. Cell Host and Microbe. https://doi.org/10.1016/j.chom.2020.09.014

Broudy, V. C. (1997). Stem cell factor and hematopoiesis. In Blood. https://doi.org/10.1182/blood.v90.4.1345

Brown, G. D. (2006). Dectin-1: A signalling non-TLR pattern-recognition receptor. In Nature Reviews Immunology. https://doi.org/10.1038/nri1745

Brown, M. A., Pierce, J. H., Watson, C. J., Falco, J., Ihle, J. N., \& Paul, W. E. (1987). B cell stimulatory factor-1/interleukin-4 mRNA is expressed by normal and transformed mast cells. Cell. https://doi.org/10.1016/0092-8674(87)90339-4

Bugl, S., Wirths, S., Müller, M. R., Radsak, M. P., \& Kopp, H. G. (2012). Current insights into neutrophil homeostasis. Annals of the New York Academy of Sciences. https://doi.org/10.1111/j.1749-6632.2012.06607.x

Cai, X., Chiu, Y. H., \& Chen, Z. J. (2014). The cGAS-cGAMP-STING pathway of cytosolic DNA sensing and signaling. In Molecular Cell (Vol. 54, Issue 2, pp. 289-296). Cell Press. https://doi.org/10.1016/j.molcel.2014.03.040

Calzada, E., Avery, E., Sam, P. N., Modak, A., Wang, C., McCaffery, J. M., Han, X., Alder, N. N., \& Claypool, S. M. (2019). Phosphatidylethanolamine made in the inner mitochondrial membrane is essential for yeast cytochrome bc 1 complex function. Nature Communications. https://doi.org/10.1038/s41467-019-09425-1

Carracedo, M., Artiach, G., Arnardottir, H., \& Bäck, M. (2019). The resolution of inflammation through omega-3 fatty acids in atherosclerosis, intimal hyperplasia, and vascular calcification. In Seminars in Immunopathology. https://doi.org/10.1007/s00281-01900767-y

Carroll, N., Elliot, J., Morton, A., \& James, A. (1993). The structure of large and small airways in nonfatal and fatal asthma. American Review of Respiratory Disease. https://doi.org/10.1164/ajrccm/147.2.405

Castela, M., Nassar, D., Sbeih, M., Jachiet, M., Wang, Z., \& Aractingi, S. (2017). Ccl2/Ccr2 signalling recruits a distinct fetal microchimeric population that rescues delayed maternal wound healing. Nature Communications. https://doi.org/10.1038/ncomms15463

Celsus, A. C. (n.d.). De medicina. Self published.

Chan, C. Y., St. John, A. L., \& Abraham, S. N. (2013). Mast Cell Interleukin-10 Drives Localized Tolerance in Chronic Bladder Infection. Immunity, 38(2), 349-359. https://doi.org/10.1016/j.immuni.2012.10.019

Chandrasoma, P., \& Taylor, C. R. (1997). Chapter 3. The Acute Inflammatory Response. In Concise Pathology.

Chang, E. Y., Guo, B., Doyle, S. E., \& Cheng, G. (2007). Cutting Edge: Involvement of the Type I IFN Production and Signaling Pathway in Lipopolysaccharide-Induced IL-10 Production. The Journal of Immunology, 178(11), 6705-6709. https://doi.org/10.4049/jimmunol.178.11.6705

Chen, E. Y., Tan, C. M., Kou, Y., Duan, Q., Wang, Z., Meirelles, G., Clark, N. R., \& Ma'ayan, A. (2013). Enrichr: interactive and collaborative HTML5 gene list enrichment analysis tool. BMC Bioinformatics, 14(1), 128. https://doi.org/10.1186/1471-2105-14-128

Cheng, N., Liang, Y., Du, X., \& Ye, R. D. (2018). Serum amyloid A promotes LPS clearance and suppresses LPS -induced inflammation and tissue injury. EMBO Reports. https://doi.org/10.15252/embr.201745517

Coccia, E. M., Uzé, G., \& Pellegrini, S. (2006). Negative regulation of type I interferon signaling. Cellular and Molecular Biology (Noisy-Le-Grand, France), 52(1), 77-87.

Collington, S. J., Williams, T. J., \& Weller, C. L. (2011). Mechanisms underlying the localisation of mast cells in tissues. In Trends in Immunology. https://doi.org/10.1016/j.it.2011.08.002

Colonna, M. (2007). TLR pathways and IFN-regulatory factors: To each its own. In European Journal of Immunology. https://doi.org/10.1002/eji.200637009

Conaway, E. A., de Oliveira, D. C., Mclnnis, C. M., Snapper, S. B., \& Horwitz, B. H. (2017). Inhibition of Inflammatory Gene Transcription by IL-10 Is Associated with Rapid Suppression of Lipopolysaccharide-Induced Enhancer Activation. The Journal of 
Immunology. https://doi.org/10.4049/jimmunol.1601781

Cougoule, C., Wiedemann, A., Lim, J., \& Caron, E. (2004). Phagocytosis, an alternative model system for the study of cell adhesion. Seminars in Cell and Developmental Biology. https://doi.org/10.1016/j.semcdb.2004.09.001

Coussens, L. M., \& Werb, Z. (2002). Inflammation and cancer. In Nature. https://doi.org/10.1038/nature01322

Crow, M. K., \& Ronnblom, L. (2019). Type i interferons in host defence and inflammatory diseases. In Lupus Science and Medicine. https://doi.org/10.1136/lupus-2019-000336

Dahlin, J. S., \& Hallgren, J. (2015). Mast cell progenitors: Origin, development and migration to tissues. Molecular Immunology, 63(1), 9-17. https://doi.org/10.1016/j.molimm.2014.01.018

Dai, H., \& Korthuis, R. J. (2011). Mast cell proteases and inflammation. In Drug Discovery Today: Disease Models. https://doi.org/10.1016/j.ddmod.2011.06.004

Daseke, M. J., Tenkorang-Impraim, M. A. A., Ma, Y., Chalise, U., Konfrst, S. R., Garrett, M. R., DeLeon-Pennell, K. Y., \& Lindsey, M. L. (2020). Exogenous IL-4 shuts off proinflammation in neutrophils while stimulating anti-inflammation in macrophages to induce neutrophil phagocytosis following myocardial infarction. Journal of Molecular and Cellular Cardiology. https://doi.org/10.1016/j.yjmcc.2020.06.006

De Filippo, K., Dudeck, A., Hasenberg, M., Nye, E., van Rooijen, N., Hartmann, K., Gunzer, M., Roers, A., \& Hogg, N. (2013). Mast cell and macrophage chemokines CXCL1/CXCL2 control the early stage of neutrophil recruitment during tissue inflammation. Blood, 121(24), 4930-4937. https://doi.org/10.1182/blood-2013-02486217

De Filippo, K., Henderson, R. B., Laschinger, M., \& Hogg, N. (2008). Neutrophil Chemokines $\mathrm{KC}$ and Macrophage-Inflammatory Protein-2 Are Newly Synthesized by Tissue Macrophages Using Distinct TLR Signaling Pathways. The Journal of Immunology, 180(6), 4308-4315. https://doi.org/10.4049/jimmunol.180.6.4308

Depinay, N., Hacini, F., Beghdadi, W., Peronet, R., \& Mécheri, S. (2006). Mast CellDependent Down-Regulation of Antigen-Specific Immune Responses by Mosquito Bites. The Journal of Immunology, 176(7), 4141-4146. https://doi.org/10.4049/jimmunol.176.7.4141

Dietrich, N., Lienenklaus, S., Weiss, S., \& Gekara, N. O. (2010). Murine Toll-Like Receptor 2 Activation Induces Type I Interferon Responses from Endolysosomal Compartments. PLoS ONE, 5(4), e10250. https://doi.org/10.1371/journal.pone.0010250

Dietrich, N., Rohde, M., Geffers, R., Kroger, A., Hauser, H., Weiss, S., \& Gekara, N. O. (2010). Mast cells elicit proinflammatory but not type I interferon responses upon activation of TLRs by bacteria. Proceedings of the National Academy of Sciences, 107(19), 8748-8753. https://doi.org/10.1073/pnas.0912551107

Dray, A. (1995). Inflammatory mediators of pain. British Journal of Anaesthesia. https://doi.org/10.1093/bja/75.2.125

Du, B., Jiang, Q.-L., Cleveland, J., Liu, B.-R., \& Zhang, D. (2016). Targeting Toll-like receptors against cancer. Journal of Cancer Metastasis and Treatment. https://doi.org/10.20517/2394-4722.2016.62

Dudeck, A., Dudeck, J., Scholten, J., Petzold, A., Surianarayanan, S., Köhler, A., Peschke, K., Vöhringer, D., Waskow, C., Krieg, T., Müller, W., Waisman, A., Hartmann, K., Gunzer, M., Roers, A., Kohler, A., Peschke, K., Vohringer, D., Waskow, C., ... Roers, A. (2011). Mast Cells Are Key Promoters of Contact Allergy that Mediate the Adjuvant Effects of Haptens. Immunity, 34(6), 973-984. https://doi.org/10.1016/j.immuni.2011.03.028

Ehrlich, P. (1878). Beiträge zur Theorie und Praxis der histologischen Färbung. In The Collected Papers of Paul Ehrlich. https://doi.org/10.1016/b978-0-08-009054-2.50006-7

Elieh Ali Komi, D., Wöhrl, S., \& Bielory, L. (2020). Mast Cell Biology at Molecular Level: a Comprehensive Review. In Clinical Reviews in Allergy and Immunology. https://doi.org/10.1007/s12016-019-08769-2

Enerbäck, L. (1966). Mast cells in rat gastrointestinal mucosa. 2. Dye-binding and metachromatic properties. Acta Pathologica et Microbiologica Scandinavica. 
https://doi.org/10.1111/apm.1966.66.3.303

Erb, K. J., Holloway, J. W., \& Le Gros, G. (1996). Innate immunity: Mast cells in the front line. Current Biology. https://doi.org/10.1016/S0960-9822(02)00632-2

Fan, J., Heller, N. M., Gorospe, M., Atasoy, U., \& Stellato, C. (2005). The role of posttranscriptional regulation in chemokine gene expression in inflammation and allergy. In European Respiratory Journal. https://doi.org/10.1183/09031936.05.00120204

Feyerabend, T. B., Gutierrez, D. A., \& Rodewald, H. R. (2016). Of Mouse Models of Mast Cell Deficiency and Metabolic Syndrome. In Cell Metabolism. https://doi.org/10.1016/j.cmet.2016.06.019

Feyerabend, T. B., Weiser, A., Tietz, A., Stassen, M., Harris, N., Kopf, M., Radermacher, P., Möller, P., Benoist, C., Mathis, D., Fehling, H. J., \& Rodewald, H. R. (2011). Cremediated cell ablation contests mast cell contribution in models of antibody- and T cellmediated autoimmunity. Immunity, 35(5), 832-844.

https://doi.org/10.1016/j.immuni.2011.09.015

Finotto, S. (2019). Resolution of allergic asthma. Seminars in Immunopathology. https://doi.org/10.1007/s00281-019-00770-3 LK -

http://ucelinks.cdlib.org:8888/sfx_local?sid=EMBASE\&sid=EMBASE\&issn=18632300\&id $=$ doi:10.1007\%2Fs00281-019-00770-

3\&atitle=Resolution+of+allergic + asthma\&stitle=Semin.Immunopathol.\&title=Seminars $+i$ $\mathrm{n}+$ Immunopathology \&volume $=41$ \&issue $=6 \&$ spage $=665$ \&epage $=674 \&$ aulast $=$ Finotto \&au first $=$ Susetta\&auinit $=S$.\&aufull=Finotto+S.\&coden $=\& i s b n=\&$ pages $=665-$ 674\&date $=2019$ \&auinit $1=$ S\&auinitm $=$

Fonteh, A. N., Samet, J. M., \& Chitton, F. H. (1995). Regulation of arachidonic acid, eicosanoid, and phospholipase A2 levels in murine mast cells by recombinant stem cell factor. Journal of Clinical Investigation. https://doi.org/10.1172/JCl118179

Fujiwara, N., \& Kobayashi, K. (2005). Macrophages in inflammation. In Current Drug Targets: Inflammation and Allergy. https://doi.org/10.2174/1568010054022024

Fullerton, J. N., \& Gilroy, D. W. (2016). Resolution of inflammation: A new therapeutic frontier. In Nature Reviews Drug Discovery. https://doi.org/10.1038/nrd.2016.39

Gabriele, L., Borghi, P., Rozera, C., Sestili, P., Andreotti, M., Guarini, A., Montefusco, E., Foà, R., \& Belardelli, F. (2004). IFN- $\alpha$ promotes the rapid differentiation of monocytes from patients with chronic myeloid leukemia into activated dendritic cells tuned to undergo full maturation after LPS treatment. Blood. https://doi.org/10.1182/blood-200303-0981

Galli, S. J., Borregaard, N., \& Wynn, T. A. (2011). Phenotypic and functional plasticity of cells of innate immunity: macrophages, mast cells and neutrophils. Nature Immunology, 12(11), 1035-1044. https://doi.org/10.1038/ni.2109

Galli, S. J., Grimbaldeston, M., \& Tsai, M. (2008). Immunomodulatory mast cells: negative, as well as positive, regulators of immunity. Nature Reviews Immunology, 8(6), 478-486. https://doi.org/10.1038/nri2327

Galli, S. J., \& Tsai, M. (2012). IgE and mast cells in allergic disease. In Nature Medicine. https://doi.org/10.1038/nm.2755

Galli, S. J., Zsebo, K. M., \& Geissler, E. N. (1993). The kit Ligand, Stem Cell Factor. Advances in Immunology. https://doi.org/10.1016/S0065-2776(08)60508-8

Gantner, B. N., Simmons, R. M., Canavera, S. J., Akira, S., \& Underhill, D. M. (2003). Collaborative induction of inflammatory responses by dectin-1 and toll-like receptor 2 . Journal of Experimental Medicine, 197(9), 1107-1117. https://doi.org/10.1084/jem.20021787

Goerdt, S., Politz, O., Schledzewski, K., Birk, R., Gratchev, A., Guillot, P., Hakiy, N., Klemke, C.-D., Dippel, E., Kodelja, V., \& Orfanos, C. E. (1999). Alternative versus Classical Activation of Macrophages. Pathobiology, 67(5-6), 222-226. https://doi.org/10.1159/000028096

González-Navajas, J. M., Lee, J., David, M., \& Raz, E. (2012). Immunomodulatory functions of type i interferons. In Nature Reviews Immunology. https://doi.org/10.1038/nri3133

Gordon, J. R., Burd, P. R., \& Galli, S. J. (1990). Mast cells as a source of multifunctional cytokines. Immunology Today, 11, 458-464. https://doi.org/10.1016/0167- 
5699(90)90176-A

Gordon, J. R., \& Galli, S. J. (1990). Mast cells as a source of both preformed and immunologically inducible TNF-a/cachectin. Nature. https://doi.org/10.1038/346274a0

Gordon, S. (2003). Alternative activation of macrophages. Nature Reviews Immunology, 3(1), 23-35. https://doi.org/10.1038/nri978

Gough, D. J., Messina, N. L., Clarke, C. J. P., Johnstone, R. W., \& Levy, D. E. (2012). Constitutive Type I Interferon Modulates Homeostatic Balance through Tonic Signaling. In Immunity. https://doi.org/10.1016/j.immuni.2012.01.011

Greenlee-Wacker, M. C. (2016). Clearance of apoptotic neutrophils and resolution of inflammation. In Immunological Reviews. https://doi.org/10.1111/imr.12453

Grimbaldeston, M. A., Nakae, S., Kalesnikoff, J., Tsai, M., \& Galli, S. J. (2007). Mast cellderived interleukin 10 limits skin pathology in contact dermatitis and chronic irradiation with ultraviolet B. Nature Immunology, 8(10), 1095-1104. https://doi.org/10.1038/ni1503

Guan, Y., Ranoa, D. R. E., Jiang, S., Mutha, S. K., Li, X., Baudry, J., \& Tapping, R. I. (2010). Human TLRs 10 and 1 Share Common Mechanisms of Innate Immune Sensing but Not Signaling. The Journal of Immunology, 184(9), 5094-5103. https://doi.org/10.4049/jimmunol.0901888

Guerrero, A. T., Zaperlon, A. C., Vieira, S. M., Pinto, L. G., Ferreira, S. H., Cunha, F. Q., Verri, W. A., \& Cunha, T. M. (2013). The role of PAF/PAFR signaling in zymosaninduced articular inflammatory hyperalgesia. Naunyn-Schmiedeberg's Archives of Pharmacology. https://doi.org/10.1007/s00210-012-0813-4

Guo, R., Zong, S., Wu, M., Gu, J., \& Yang, M. (2017). Architecture of Human Mitochondrial Respiratory Megacomplex I2IIIIIV2. Cell. https://doi.org/10.1016/j.cell.2017.07.050

Hahnefeld, L., Gurke, R., Thomas, D., Schreiber, Y., Schäfer, S. M. G., Trautmann, S., Snodgrass, I. F., Kratz, D., Geisslinger, G., \& Ferreirós, N. (2019). Implementation of lipidomics in clinical routine: Can fluoride/citrate blood sampling tubes improve preanalytical stability? Talanta, 120593. https://doi.org/https://doi.org/10.1016/j.talanta.2019.120593

Hahnefeld, L., Kornstädt, L., Kratz, D., Ferreirós, N., Geisslinger, G., Pierre, S., \& Scholich, K. (2021). Lipidomic analysis of local inflammation models shows a specific systemic acute phase response to lipopolysaccharides. Biochimica et Biophysica Acta (BBA) Molecular and Cell Biology of Lipids, 1866(1), 158822. https://doi.org/10.1016/j.bbalip.2020.158822

Hakim, M. S., Chen, S., Ding, S., Yin, Y., Ikram, A., Ma, X. X., Wang, W., Peppelenbosch, M. P., \& Pan, Q. (2018). Basal interferon signaling and therapeutic use of interferons in controlling rotavirus infection in human intestinal cells and organoids. Scientific Reports. https://doi.org/10.1038/s41598-018-26784-9

Hamilton, J. A., Whitty, G. A., Kola, I., \& Hertzog, P. J. (1996). Endogenous IFN- $\alpha \beta$ suppresses colony-stimulating factor (CSF)-1-stimulated macrophage DNA synthesis and mediates inhibitory effects of lipopolysaccharide and TNF-a. Journal of Immunology.

Hammel, I., Lagunoff, D., \& Krüger, P. G. (1989). Recovery of rat mast cells after secretion: A morphometric study. Experimental Cell Research. https://doi.org/10.1016/00144827(89)90349-2

Harizi, H., \& Gualde, N. (2006). Pivotal role of PGE2 and IL-10 in the cross-regulation of dendritic cell-derived inflammatory mediators. Cellular \& Molecular Immunology, 3(4), 271-277.

Hart, P. H., Vitti, G. F., Burgess, D. R., Whitty, G. A., Piccoli, D. S., \& Hamilton, J. A. (1989). Potential antiinflammatory effects of interleukin 4: suppression of human monocyte tumor necrosis factor alpha, interleukin 1, and prostaglandin E2. Proceedings of the National Academy of Sciences, 86(10), 3803-3807.

https://doi.org/10.1073/pnas.86.10.3803

Hayashi, S. ichi, Kunisada, T., Ogawa, M., Yamaguchi, K., \& Nishikawa, S. ichi. (1991). Exon skipping by mutation of an authentic splice site of c-kit gene in W/W mouse. Nucleic Acids Research, 19(6), 1267-1271. https://doi.org/10.1093/nar/19.6.1267

He, Y., Ou, Z., Chen, X., Zu, X., Liu, L., Li, Y., Cao, Z., Chen, M., Chen, Z., Chen, H., Qi, L., 
\& Wang, L. (2016). LPS/TLR4 signaling enhances TGF- $\beta$ response through downregulating BAMBI during prostatic hyperplasia. Scientific Reports. https://doi.org/10.1038/srep27051

Hedl, M., \& Abraham, C. (2012). Nod2-induced autocrine interleukin-1 alters signaling by ERK and p38 to differentially regulate secretion of inflammatory cytokines. Gastroenterology. https://doi.org/10.1053/j.gastro.2012.08.048

Heim, M. H., Kerr, I. M., Stark, G. R., \& Darnell, J. E. (1995). Contribution of STAT SH2 groups to specific interferon signaling by the Jak-STAT pathway. Science. https://doi.org/10.1126/science.7871432

Heinrich, M. C., Dooley, D. C., Freed, A. C., Band, L., Hoatlin, M. E., Keeble, W. W., Peters, S. T., Silvey, K. V., Ey, F. S., Kabat, D., Maziarz, R. T., \& Bagby, G. C. (1993). Constitutive expression of steel factor gene by human stromal cells. Blood. https://doi.org/10.1182/blood.v82.3.771.771

Hermann, P., Rubio, M., Nakajima, T., Delespesse, G., \& Sarfati, M. (1998). IFN-alpha priming of human monocytes differentially regulates gram-positive and gram-negative bacteria-induced IL-10 release and selectively enhances IL-12p70, CD80, and MHC class I expression. Journal of Immunology (Baltimore, Md. : 1950).

Hibbert, L., Pflanz, S., De Waal Malefyt, R., \& Kastelein, R. A. (2003). IL-27 and IFN-a signal via Stat 1 and Stat3 and induce T-Bet and IL-12Rß2 in naive T cells. Journal of Interferon and Cytokine Research. https://doi.org/10.1089/10799900360708632

Hills, R., Ishii, S., Jancar, S., Mclntyre, T., Ninio, E., O'Neill, C., Rios, F. J. O., Travers, J. B., \& Whittaker, M. (2019). Platelet-activating factor receptor (version 2019.4) in the IUPHAR/BPS Guide to Pharmacology Database. IUPHAR/BPS Guide to Pharmacology CITE, 2019(4 SE-Summaries). https://doi.org/10.2218/gtopdb/F55/2019.4

Honda, K., Ohba, Y., Yanai, H., Hegishi, H., Mizutani, T., Takaoka, A., Taya, C., \& Taniguchi, T. (2005). Spatiotemporal regulation of MyD88-IRF-7 signalling for robust type-I interferon induction. Nature, 434(7036), 1035-1040. https://doi.org/10.1038/nature03547

Honda, K., Yanai, H., Mizutani, T., Negishi, H., Shimada, N., Suzuki, N., Ohba, Y., Takaoka, A., Yeh, W. C., \& Taniguchi, T. (2004). Role of a transductional-transcriptional processor complex involving MyD88 and IRF-7 in Toll-like receptor signaling. Proceedings of the National Academy of Sciences of the United States of America. https://doi.org/10.1073/pnas.0406933101

Honda, K., Yanai, H., Negishi, H., Asagiri, M., Sato, M., Mizutani, T., Shimada, N., Ohba, Y., Takaoka, A., Yoshida, N., \& Taniguchi, T. (2005). IRF-7 is the master regulator of type-I interferon-dependent immune responses. Nature, 434(7034), 772-777. https://doi.org/10.1038/nature03464

Honda, K., Yanai, H., Takaoka, A., \& Taniguchi, T. (2005). Regulation of the type I IFN induction: A current view. In International Immunology. https://doi.org/10.1093/intimm/dxh318

Hoshino, K., Takeuchi, O., Kawai, T., Sanjo, H., Ogawa, T., Takeda, Y., Takeda, K., \& Akira, S. (1999). Cutting Edge: Toll-Like Receptor 4 (TLR4)-Deficient Mice Are Hyporesponsive to Lipopolysaccharide: Evidence for TLR4 as the \&lt;em\&gt;Lps\&lt;/em\&gt; Gene Product. The Journal of Immunology, 162(7), 3749 LP _ 3752. http://www.jimmunol.org/content/162/7/3749.abstract

Hotamisligil, G. S. (2006). Inflammation and metabolic disorders. In Nature. https://doi.org/10.1038/nature05485

Hotamisligil, G. S. (2017). Inflammation, metaflammation and immunometabolic disorders. In Nature. https://doi.org/10.1038/nature21363

Ikeda, Y., Adachi, Y., Ishii, T., Miura, N., Tamura, H., \& Ohno, N. (2008). Dissociation of tolllike receptor 2-mediated innate immune response to zymosan by organic solventtreatment without loss of dectin-1 reactivity. Biological and Pharmaceutical Bulletin, 31(1), 13-18. https://doi.org/10.1248/bpb.31.13

Ip, W. K. E., Hoshi, N., Shouval, D. S., Snapper, S., \& Medzhitov, R. (2017). Antiinflammatory effect of IL-10 mediated by metabolic reprogramming of macrophages. Science. https://doi.org/10.1126/science.aal3535 
Irani, A. M., \& Schwartz, L. B. (1994). Human mast cell heterogeneity. In Allergy Proceedings. https://doi.org/10.2500/108854194778816472

Isaacs, A., \& Lindenmann, J. (1957). Virus interference. I. The interferon. Proceedings of the Royal Society of London. Series B - Biological Sciences, 147(927), 258-267. https://doi.org/10.1098/rspb.1957.0048

Italiani, P., \& Boraschi, D. (2014). From monocytes to M1/M2 macrophages: Phenotypical vs. functional differentiation. In Frontiers in Immunology. https://doi.org/10.3389/fimmu.2014.00514

Ivashkiv, L. B., \& Donlin, L. T. (2014). Regulation of type I interferon responses. Nature Reviews Immunology, 14(1), 36-49. https://doi.org/10.1038/nri3581

Jachetti, E., D'Incà, F., Danelli, L., Magris, R., Dal Secco, C., Vit, F., Cancila, V., Tripodo, C., Scapini, P., Colombo, M. P., Pucillo, C., \& Frossi, B. (2019). Frontline Science: Mast cells regulate neutrophil homeostasis by influencing macrophage clearance activity. Journal of Leukocyte Biology, 105(4), 633-644. https://doi.org/10.1002/JLB.4HI1018390R

Jia, X. M., Tang, B., Zhu, L. Le, Liu, Y. H., Zhao, X. Q., Gorjestani, S., Hsu, Y. M. S., Yang, L., Guan, J. H., Xu, G. T., \& Lin, X. (2014). CARD9 mediates Dectin-1-induced ERK activation by linking Ras-GRF1 to H-Ras for antifungal immunity. Journal of Experimental Medicine. https://doi.org/10.1084/jem.20132349

Kagan, J. C., Su, T., Horng, T., Chow, A., Akira, S., \& Medzhitov, R. (2008). TRAM couples endocytosis of Toll-like receptor 4 to the induction of interferon- $\beta$. Nature Immunology, 9(4), 361-368. https://doi.org/10.1038/ni1569

Kawai, T., Sato, S., Ishii, K. J., Coban, C., Hemmi, H., Yamamoto, M., Terai, K., Matsuda, M., Inoue, J. I., Uematsu, S., Takeuchi, O., \& Akira, S. (2004). Interferon- $\alpha$ induction through Toll-like receptors involves a direct interaction of IRF7 with MyD88 and TRAF6. Nature Immunology. https://doi.org/10.1038/ni1118

Kawasaki, T., \& Kawai, T. (2014). Toll-like receptor signaling pathways. In Frontiers in Immunology. https://doi.org/10.3389/fimmu.2014.00461

Keck, S., Müller, I., Fejer, G., Savic, I., Tchaptchet, S., Nielsen, P. J., Galanos, C., Huber, M., \& Freudenberg, M. A. (2011). Absence of TRIF Signaling in LipopolysaccharideStimulated Murine Mast Cells. The Journal of Immunology, 186(9), 5478-5488. https://doi.org/10.4049/jimmunol.1000458

Kern, K., Schäfer, S. M. G., Cohnen, J., Pierre, S., Osthues, T., Tarighi, N., Hohmann, S., Ferreiros, N., Brüne, B., Weigert, A., Geisslinger, G., Sisignano, M., \& Scholich, K. (2018). The G2A Receptor Controls Polarization of Macrophage by Determining Their Localization Within the Inflamed Tissue. Frontiers in Immunology, 9, 2261. https://doi.org/10.3389/fimmu.2018.02261

Kiesslich, R., Fritsch, J., Holtmann, M., Koehler, H. H., Stolte, M., Kanzler, S., Nafe, B., Jung, M., Galle, P. R., \& Neurath, M. F. (2003). Methylene blue-aided chromoendoscopy for the detection of intraepithelial neoplasia and colon cancer in ulcerative colitis. Gastroenterology. https://doi.org/10.1053/gast.2003.50146

Korns, D., Frasch, S. C., Fernandez-Boyanapalli, R., Henson, P. M., \& Bratton, D. L. (2011). Modulation of macrophage efferocytosis in inflammation. In Frontiers in Immunology. https://doi.org/10.3389/fimmu.2011.00057

Kornstädt, L., Pierre, S., Weigert, A., Ebersberger, S., Schäufele, T. J., Kolbinger, A., Schmid, T., Cohnen, J., Brüne, B., Ebersberger, I., Thomas, D., Ferreiro, N., \& Scholich, K. (2021). Bacterial and Fungal Toll-Like Receptor Activation Elicits Type I IFN Responses in Mast Cells. Frontiers in Immunology, 11(February), 3872. https://doi.org/10.3389/fimmu.2020.607048

Kotenko, S. V, Gallagher, G., Baurin, V. V, Lewis-Antes, A., Shen, M., Shah, N. K., Langer, J. A., Sheikh, F., Dickensheets, H., \& Donnelly, R. P. (2003). IFN- $\lambda$ s mediate antiviral protection through a distinct class II cytokine receptor complex. Nature Immunology, 4(1), 69-77. https://doi.org/10.1038/ni875

Kourtzelis, I., Hajishengallis, G., \& Chavakis, T. (2020). Phagocytosis of Apoptotic Cells in Resolution of Inflammation. In Frontiers in Immunology. https://doi.org/10.3389/fimmu.2020.00553 
Kraneveld, A. D., Sagar, S., Garssen, J., \& Folkerts, G. (2012). The two faces of mast cells in food allergy and allergic asthma: The possible concept of Yin Yang. In Biochimica et Biophysica Acta - Molecular Basis of Disease.

https://doi.org/10.1016/j.bbadis.2011.06.013

Kugelberg, E. (2016). Cell death: Find me and eat me. In Nature Reviews Immunology. https://doi.org/10.1038/nri.2016.23

Kuleshov, M. V, Jones, M. R., Rouillard, A. D., Fernandez, N. F., Duan, Q., Wang, Z., Koplev, S., Jenkins, S. L., Jagodnik, K. M., Lachmann, A., McDermott, M. G., Monteiro, C. D., Gundersen, G. W., \& Ma'ayan, A. (2016). Enrichr: a comprehensive gene set enrichment analysis web server 2016 update. Nucleic Acids Research, 44(W1), W90W97. https://doi.org/10.1093/nar/gkw377

Kumar, H., Kawai, T., \& Akira, S. (2009). Pathogen recognition in the innate immune response. In Biochemical Journal. https://doi.org/10.1042/BJ20090272

Kumaran Satyanarayanan, S., El Kebir, D., Soboh, S., Butenko, S., Sekheri, M., Saadi, J., Peled, N., Assi, S., Othman, A., Schif-Zuck, S., Feuermann, Y., Barkan, D., Sher, N., Filep, J. G., \& Ariel, A. (2019). IFN- $\beta$ is a macrophage-derived effector cytokine facilitating the resolution of bacterial inflammation. Nature Communications, 10(1), 3471. https://doi.org/10.1038/s41467-019-10903-9

Landskron, G., De La Fuente, M., Thuwajit, P., Thuwajit, C., \& Hermoso, M. A. (2014). Chronic inflammation and cytokines in the tumor microenvironment. In Journal of Immunology Research. https://doi.org/10.1155/2014/149185

Lange, C., Nett, J. H., Trumpower, B. L., \& Hunte, C. (2001). Specific roles of proteinphospholipid interactions in the yeast cytochrome bc1 complex structure. EMBO Journal. https://doi.org/10.1093/emboj/20.23.6591

Lauber, K., Bohn, E., Kröber, S. M., Xiao, Y. J., Blumenthal, S. G., Lindemann, R. K., Marini, P., Wiedig, C., Zobywalski, A., Baksh, S., Xu, Y., Autenrieth, I. B., Schulze-Osthoff, K., Belka, C., Stuhler, G., \& Wesselborg, S. (2003). Apoptotic cells induce migration of phagocytes via caspase-3-mediated release of a lipid attraction signal. Cell. https://doi.org/10.1016/S0092-8674(03)00422-7

Law, S. H., Chan, M. L., Marathe, G. K., Parveen, F., Chen, C. H., \& Ke, L. Y. (2019). An updated review of lysophosphatidylcholine metabolism in human diseases. In International Journal of Molecular Sciences. https://doi.org/10.3390/ijms20051149

Lazear, H. M., Schoggins, J. W., \& Diamond, M. S. (2019). Shared and Distinct Functions of Type I and Type III Interferons. In Immunity. https://doi.org/10.1016/j.immuni.2019.03.025

Lech, M., \& Anders, H. J. (2013). Macrophages and fibrosis: How resident and infiltrating mononuclear phagocytes orchestrate all phases of tissue injury and repair. In Biochimica et Biophysica Acta - Molecular Basis of Disease. https://doi.org/10.1016/j.bbadis.2012.12.001

Lee, A. J., \& Ashkar, A. A. (2018). The dual nature of type I and type II interferons. In Frontiers in Immunology. https://doi.org/10.3389/fimmu.2018.02061

Lee, C. G. L., \& O'Brien, W. E. (1995). A unique member of the thymidylate kinase family that is induced during macrophage activation. Journal of Immunology (Baltimore, Md. : 1950).

Lee, H., Lee, I. S., \& Choue, R. (2013). Obesity, inflammation and diet. In Pediatric Gastroenterology, Hepatology and Nutrition. https://doi.org/10.5223/pghn.2013.16.3.143

Lee, S. J., Evers, S., Roeder, D., Parlow, A. F., Risteli, J., Risteli, L., Lee, Y. C., Feizi, T., Langen, H., \& Nussenzweig, M. C. (2002). Mannose receptor-mediated regulation of serum glycoprotein homeostasis. Science. https://doi.org/10.1126/science.1069540

Levine, J. H., Simonds, E. F., Bendall, S. C., Davis, K. L., Amir, E. D., Tadmor, M. D., Litvin, O., Fienberg, H. G., Jager, A., Zunder, E. R., Finck, R., Gedman, A. L., Radtke, I., Downing, J. R., Pe'er, D., \& Nolan, G. P. (2015). Data-Driven Phenotypic Dissection of AML Reveals Progenitor-like Cells that Correlate with Prognosis. Cell, 162(1), 184-197. https://doi.org/10.1016/j.cell.2015.05.047

Levy, B. D., Clish, C. B., Schmidt, B., Gronert, K., \& Serhan, C. N. (2001). Lipid mediator class switching during acute inflammation: signals in resolution. Nature Immunology, 
2(7), 612-619. https://doi.org/10.1038/89759

Levy, D. E., Marié, I. J., \& Durbin, J. E. (2011). Induction and function of type I and III interferon in response to viral infection. Current Opinion in Virology, 1(6), 476-486. https://doi.org/10.1016/j.coviro.2011.11.001

Li, S. F., Gong, M. J., Zhao, F. R., Shao, J. J., Xie, Y. L., Zhang, Y. G., \& Chang, H. Y. (2018). Type i interferons: Distinct biological activities and current applications for viral infection. In Cellular Physiology and Biochemistry. https://doi.org/10.1159/000495897

Lilla, J. N., Chen, C. G., Mukai, K., BenBarak, M. J., Franco, C. B., Kalesnikoff, J., Yu, M., Tsai, M., Piliponsky, A. M., \& Galli, S. J. (2011). Reduced mast cell and basophil numbers and function in Cpa3-Cre; Mcl-1 fl/fl mice. Blood, 118(26), 6930-6938. https://doi.org/10.1182/blood-2011-03-343962

López-Collazo, E., Hortelano, S., Rojas, A., \& Boscá, L. (1998). Triggering of peritoneal macrophages with IFN- $\alpha / \beta$ attenuates the expression of inducible nitric oxide synthase through a decrease in NF-KB activation. The Journal of Immunology.

Luft, T., Pang, K. C., Thomas, E., Hertzog, P., Hart, D. N., Trapani, J., \& Cebon, J. (1998). Type I IFNs enhance the terminal differentiation of dendritic cells. Journal of Immunology (Baltimore, Md. : 1950).

Lund, S., Christensen, K. V., Hedtjärn, M., Mortensen, A. L., Hagberg, H., Falsig, J., Hasseldam, H., Schrattenholz, A., Pörzgen, P., \& Leist, M. (2006). The dynamics of the LPS triggered inflammatory response of murine microglia under different culture and in vivo conditions. Journal of Neuroimmunology. https://doi.org/10.1016/j.jneuroim.2006.07.007

Maderna, P., \& Godson, C. (2003). Phagocytosis of apoptotic cells and the resolution of inflammation. In Biochimica et Biophysica Acta - Molecular Basis of Disease. https://doi.org/10.1016/j.bbadis.2003.09.004

Malakhova, O. A., Kim, K. I. I., Luo, J.-K., Zou, W., Kumar, K. G. S., Fuchs, S. Y., Shuai, K., \& Zhang, D.-E. (2006). UBP43 is a novel regulator of interferon signaling independent of its ISG15 isopeptidase activity. The EMBO Journal, 25(11), 2358-2367. https://doi.org/10.1038/sj.emboj.7601149

Mantovani, A., Sica, A., Sozzani, S., Allavena, P., Vecchi, A., \& Locati, M. (2004). The chemokine system in diverse forms of macrophage activation and polarization. In Trends in Immunology. https://doi.org/10.1016/j.it.2004.09.015

Marie, I. (1998). Differential viral induction of distinct interferon-alpha genes by positive feedback through interferon regulatory factor-7. The EMBO Journal. https://doi.org/10.1093/emboj/17.22.6660

Marshall, J. S., Portales-Cervantes, L., \& Leong, E. (2019). Mast cell responses to viruses and pathogen products. In International Journal of Molecular Sciences. https://doi.org/10.3390/ijms20174241

Martinez, F. O., Helming, L., \& Gordon, S. (2009). Alternative activation of macrophages: An immunologic functional perspective. In Annual Review of Immunology. https://doi.org/10.1146/annurev.immunol.021908.132532

Matsuyama, T., Kimura, T., Kitagawa, M., Pfeffer, K., Kawakami, T., Watanabe, N., Kündig, T. M., Amakawa, R., Kishihara, K., Wakeham, A., Potter, J., Furlonger, C. L., Narendran, A., Suzuki, H., Ohashi, P. S., Paige, C. J., Taniguchi, T., \& Mak, T. W. (1993). Targeted disruption of IRF-1 or IRF-2 results in abnormal type I IFN gene induction and aberrant lymphocyte development. Cell, 75(1), 83-97. https://doi.org/10.1016/S0092-8674(05)80086-8

McCormick, S. M., \& Heller, N. M. (2015). Commentary: IL-4 and IL-13 receptors and signaling. Cytokine. https://doi.org/10.1016/j.cyto.2015.05.023

Mclnnes, I. B., \& Schett, G. (2017). Pathogenetic insights from the treatment of rheumatoid arthritis. In The Lancet. https://doi.org/10.1016/S0140-6736(17)31472-1

McLeod, J. J. A., Baker, B., \& Ryan, J. J. (2015). Mast cell production and response to IL-4 and IL-13. Cytokine, 75(1), 57-61. https://doi.org/10.1016/j.cyto.2015.05.019

McNab, F., Mayer-Barber, K., Sher, A., Wack, A., \& O'Garra, A. (2015). Type I interferons in infectious disease. In Nature Reviews Immunology. https://doi.org/10.1038/nri3787

McQuin, C., Goodman, A., Chernyshev, V., Kamentsky, L., Cimini, B. A., Karhohs, K. W., 
Doan, M., Ding, L., Rafelski, S. M., Thirstrup, D., Wiegraebe, W., Singh, S., Becker, T., Caicedo, J. C., \& Carpenter, A. E. (2018). CellProfiler 3.0: Next-generation image processing for biology. PLoS Biology, 16(7), e2005970. https://doi.org/10.1371/journal.pbio.2005970

Medrano, R. F. V., Hunger, A., Mendonça, S. A., Barbuto, J. A. M., \& Strauss, B. E. (2017). Immunomodulatory and antitumor effects of type I interferons and their application in cancer therapy. In Oncotarget. https://doi.org/10.18632/oncotarget.19531

Medzhitov, R. (2008). Origin and physiological roles of inflammation. In Nature. https://doi.org/10.1038/nature07201

Meng, Z., Yan, C., Deng, Q., Dong, X., Duan, Z. M., Gao, D. F., \& Niu, X. L. (2013). Oxidized low-density lipoprotein induces inflammatory responses in cultured human mast cells via toll-like receptor 4. Cellular Physiology and Biochemistry. https://doi.org/10.1159/000350102

Mita, H., Ishii, T., \& Akiyama, K. (1999). Generation of thromboxane A2 from highly purified human sinus mast cells after immunological stimulation. Prostaglandins Leukotrienes and Essential Fatty Acids. https://doi.org/10.1054/plef.1999.0022

Miyamoto, M., Fujita, T., Kimura, Y., Maruyama, M., Harada, H., Sudo, Y., Miyata, T., \& Taniguchi, T. (1988). Regulated expression of a gene encoding a nuclear factor, IRF-1, that specifically binds to IFN- $\beta$ gene regulatory elements. Cell. https://doi.org/10.1016/S0092-8674(88)91307-4

Moalem, G., \& Tracey, D. J. (2006). Immune and inflammatory mechanisms in neuropathic pain. In Brain Research Reviews. https://doi.org/10.1016/j.brainresrev.2005.11.004

Mogensen, T. H. (2009). Pathogen recognition and inflammatory signaling in innate immune defenses. In Clinical Microbiology Reviews. https://doi.org/10.1128/CMR.00046-08

Molteni, M., Gemma, S., \& Rossetti, C. (2016). The Role of Toll-Like Receptor 4 in Infectious and Noninfectious Inflammation. In Mediators of Inflammation. https://doi.org/10.1155/2016/6978936

Moon, T. C., Dean Befus, A., \& Kulka, M. (2014). Mast cell mediators: Their differential release and the secretory pathways involved. In Frontiers in Immunology. https://doi.org/10.3389/fimmu.2014.00569

Mukai, K., Tsai, M., Saito, H., \& Galli, S. J. (2018). Mast cells as sources of cytokines, chemokines, and growth factors. In Immunological Reviews. https://doi.org/10.1111/imr.12634

Mukherjee, S., Karmakar, S., \& Babu, S. P. S. (2016). TLR2 and TLR4 mediated host immune responses in major infectious diseases: A review. In Brazilian Journal of Infectious Diseases. https://doi.org/10.1016/j.bjid.2015.10.011

Nakano, T., Sonoda, T., Hayashi, C., Yamatodani, A., Kanayama, Y., Yamamura, T., Asai, H., Yonezawa, T., Kitamura, Y., \& Galli, S. J. (1985). Fate of bone marrow-derived cultured mast cells after intracutaneous, intraperitoneal, and intravenous transfer into genetically mast cell-deficient W/Wv mice: Evidence that cultured mast cells can give rise to both connective tissue type and mucosal mast. Journal of Experimental Medicine. https://doi.org/10.1084/jem.162.3.1025

Neurath, M. F., \& Leppkes, M. (2019). Resolution of ulcerative colitis. Seminars in Immunopathology, 41(6), 747-756. https://doi.org/10.1007/s00281-019-00751-6

Nigrovic, P. A., Gray, D. H. D., Jones, T., Hallgren, J., Kuo, F. C., Chaletzky, B., Gurish, M., Mathis, D., Benoist, C., \& Lee, D. M. (2008). Genetic inversion in mast cell-deficient Wsh mice interrupts corin and manifests as hematopoietic and cardiac aberrancy. American Journal of Pathology, 173(6), 1693-1701. https://doi.org/10.2353/ajpath.2008.080407

Ning, S., Pagano, J. S., \& Barber, G. N. (2011). IRF7: Activation, regulation, modification and function. In Genes and Immunity. https://doi.org/10.1038/gene.2011.21

Nishi, C., Toda, S., Segawa, K., \& Nagata, S. (2014). Tim4- and MerTK-Mediated Engulfment of Apoptotic Cells by Mouse Resident Peritoneal Macrophages. Molecular and Cellular Biology. https://doi.org/10.1128/mcb.01394-13

Nocka, K., Tan, J. C., Chiu, E., Chu, T. Y., Ray, P., Traktman, P., \& Besmer, P. (1990). Molecular bases of dominant negative and loss of function mutations at the murine $c$ - 
kit/white spotting locus: W37, W(v), W41 and W. EMBO Journal, 9(6), 1805-1813. https://doi.org/10.1002/j.1460-2075.1990.tb08305.x

O'Neill, L. A. J., Golenbock, D., \& Bowie, A. G. (2013). The history of Toll-like receptorsredefining innate immunity. In Nature Reviews Immunology.

https://doi.org/10.1038/nri3446

Odendall, C., Voak, A. A., \& Kagan, J. C. (2017). Type III IFNs Are Commonly Induced by Bacteria-Sensing TLRs and Reinforce Epithelial Barriers during Infection. The Journal of Immunology. https://doi.org/10.4049/jimmunol.1700250

Oganesyan, G., Saha, S. K., Guo, B., He, J. Q., Shahangian, A., Zarnegar, B., Perry, A., \& Cheng, G. (2006). Critical role of TRAF3 in the Toll-like receptor-dependent and independent antiviral response. Nature. https://doi.org/10.1038/nature04374

Okayama, Y., \& Kawakami, T. (2006). Development, migration, and survival of mast cells. In Immunologic Research. https://doi.org/10.1385/IR:34:2:97

Okumura, S., Kashiwakura, J. I., Tomita, H., Matsumoto, K., Nakajima, T., Saito, H., \& Okayama, Y. (2003). Identification of specific gene expression profiles in human mast cells mediated by Toll-like receptor 4 and FcERI. Blood. https://doi.org/10.1182/blood2002-12-3929

Oliveira-Nascimento, L., Massari, P., \& Wetzler, L. M. (2012). The role of TLR2 ininfection and immunity. In Frontiers in Immunology. https://doi.org/10.3389/fimmu.2012.00079

Olivera, A., \& Rivera, J. (2011). An emerging role for the lipid mediator sphingosine-1phosphate in mast cell effector function and allergic disease. Advances in Experimental Medicine and Biology. https://doi.org/10.1007/978-1-4419-9533-9_8

Ortega-Gómez, A., Perretti, M., \& Soehnlein, O. (2013). Resolution of inflammation: An integrated view. In EMBO Molecular Medicine. https://doi.org/10.1002/emmm.201202382

Otsuka, A., Kubo, M., Honda, T., Egawa, G., Nakajima, S., Tanizaki, H., Kim, B., Matsuoka, S., Watanabe, T., Nakae, S., Miyachi, Y., \& Kabashima, K. (2011). Requirement of Interaction between Mast Cells and Skin Dendritic Cells to Establish Contact Hypersensitivity. PLoS ONE, 6(9), e25538. https://doi.org/10.1371/journal.pone.0025538

Padawer, J. (1974). Mast cells: Extended lifespan and lack of granule turnover under normal in vivo conditions. Experimental and Molecular Pathology. https://doi.org/10.1016/00144800(74)90059-8

Paul, A., Tang, T. H., \& Ng, S. K. (2018). Interferon regulatory factor 9 structure and regulation. In Frontiers in Immunology. https://doi.org/10.3389/fimmu.2018.01831

Peirce, J. M., \& Alviña, K. (2019). The role of inflammation and the gut microbiome in depression and anxiety. In Journal of Neuroscience Research. https://doi.org/10.1002/jnr.24476

Pejler, G., Åbrink, M., Ringvall, M., \& Wernersson, S. (2007). Mast Cell Proteases. In Advances in Immunology. https://doi.org/10.1016/S0065-2776(07)95006-3

Pérez-Rodríguez, M. J., Ibarra-Sánchez, A., Román-Figueroa, A., Pérez-Severiano, F., \& González-Espinosa, C. (2020). Mutant Huntingtin affects toll-like receptor 4 intracellular trafficking and cytokine production in mast cells. Journal of Neuroinflammation. https://doi.org/10.1186/s12974-020-01758-9

Perkins, D. J., \& Vogel, S. N. (2015). Space and time: New considerations about the relationship between Toll-like receptors (TLRs) and type I interferons (IFNs). In Cytokine. https://doi.org/10.1016/j.cyto.2015.03.001

Pierre, S., Linke, B., Suo, J., Tarighi, N., Del Turco, D., Thomas, D., Ferreiros, N., Stegner, D., Frölich, S., Sisignano, M., Meyer Dos Santos, S., DeBruin, N., Nüsing, R. M., Deller, T., Nieswandt, B., Geisslinger, G., \& Scholich, K. (2017). GPVI and Thromboxane Receptor on Platelets Promote Proinflammatory Macrophage Phenotypes during Cutaneous Inflammation. Journal of Investigative Dermatology, 137(3), 686-695. https://doi.org/10.1016/j.jid.2016.09.036

Pierre, S., \& Scholich, K. (2010). Toponomics: Studying protein-protein interactions and protein networks in intact tissue. Molecular BioSystems, 6(4), 641-647. https://doi.org/10.1039/b910653g 
Poltorak, A., He, X., Smirnova, I., Liu, M. Y., Van Huffel, C., Du, X., Birdwell, D., Alejos, E., Silva, M., Galanos, C., Freudenberg, M., Ricciardi-Castagnoli, P., Layton, B., \& Beutler, B. (1998). Defective LPS signaling in C3H/HeJ and C57BL/10ScCr mice: Mutations in Tlr4 gene. Science, 282(5396), 2085-2088. https://doi.org/10.1126/science.282.5396.2085

Prame Kumar, K., Nicholls, A. J., \& Wong, C. H. Y. (2018). Partners in crime: neutrophils and monocytes/macrophages in inflammation and disease. In Cell and Tissue Research. https://doi.org/10.1007/s00441-017-2753-2

Qin, X., Qiu, C., \& Zhao, L. (2014). Lysophosphatidylcholine perpetuates macrophage polarization toward classically activated phenotype in inflammation. Cellular Immunology, 289(1-2), 185-190. https://doi.org/10.1016/j.cellimm.2014.04.010 PM 24841857

Reber, L. L., Marichal, T., \& Galli, S. J. (2012). New models for analyzing mast cell functions in vivo. In Trends in Immunology (Vol. 33, Issue 12, pp. 613-625). NIH Public Access. https://doi.org/10.1016/j.it.2012.09.008

Reber, L. L., Sibilano, R., Starkl, P., Roers, A., Grimbaldeston, M. A., Tsai, M., Gaudenzio, N., \& Galli, S. J. (2017). Imaging protective mast cells in living mice during severe contact hypersensitivity. JCI Insight, 2(9). https://doi.org/10.1172/jci.insight.92900

Reid, D. M., Gow, N. A., \& Brown, G. D. (2009). Pattern recognition: recent insights from Dectin-1. In Current Opinion in Immunology. https://doi.org/10.1016/j.coi.2009.01.003

Rennick, D., Hunte, B., Holland, G., \& Thompson-Snipes, L. A. (1995). Cofactors are essential for stem cell factor-dependent growth and maturation of mast cell progenitors: Comparative effects of interleukin-3 (IL-3), IL-4, IL-10, and fibroblasts. Blood. https://doi.org/10.1182/blood.v85.1.57.bloodjournal85157

Ribatti, D. (2018). The Staining of Mast Cells: A Historical Overview. In International Archives of Allergy and Immunology. https://doi.org/10.1159/000487538

Ricciotti, E., \& Fitzgerald, G. A. (2011). Prostaglandins and inflammation. Arteriosclerosis, Thrombosis, and Vascular Biology. https://doi.org/10.1161/ATVBAHA.110.207449

Rodewald, H. R., \& Feyerabend, T. B. (2012). Widespread Immunological Functions of Mast Cells: Fact or Fiction? In Immunity (Vol. 37, Issue 1, pp. 13-24). Immunity. https://doi.org/10.1016/j.immuni.2012.07.007

Rollag, H., Degre, M., \& Sonnenfeld, G. (1984). Effects of Interferon- $\alpha / \beta$ and Interferon- $\gamma$ Preparations on Phagocytosis by Mouse Peritoneal Macrophages. Scandinavian Journal of Immunology. https://doi.org/10.1111/j.1365-3083.1984.tb00988.x

Ronchetti, S., Migliorati, G., \& Delfino, D. V. (2017). Association of inflammatory mediators with pain perception. In Biomedicine and Pharmacotherapy. https://doi.org/10.1016/j.biopha.2017.12.001

Ruiz, F., Vigne, S., \& Pot, C. (2019). Resolution of inflammation during multiple sclerosis. In Seminars in Immunopathology. https://doi.org/10.1007/s00281-019-00765-0

Sabat, R., Grütz, G., Warszawska, K., Kirsch, S., Witte, E., Wolk, K., \& Geginat, J. (2010). Biology of interleukin-10. Cytokine and Growth Factor Reviews. https://doi.org/10.1016/j.cytogfr.2010.09.002

Saijo, S., \& Iwakura, Y. (2011). Dectin-1 and Dectin-2 in innate immunity against fungi. International Immunology. https://doi.org/10.1093/intimm/dxr046

Salerno, F., Guislain, A., Cansever, D., \& Wolkers, M. C. (2016). TLR-Mediated Innate Production of IFN-y by CD8 + T Cells Is Independent of Glycolysis. The Journal of Immunology. https://doi.org/10.4049/jimmunol.1501997

Saluja, R., Kumar, A., Jain, M., Goel, S. K., \& Jain, A. (2017). Role of sphingosine-1phosphate in mast cell functions and asthma and its regulation by non-coding RNA. In Frontiers in Immunology. https://doi.org/10.3389/fimmu.2017.00587

Sandig, H., \& Bulfone-Paus, S. (2012a). TLR signaling in mast cells: Common and unique features. In Frontiers in Immunology. https://doi.org/10.3389/fimmu.2012.00185

Sandig, H., \& Bulfone-Paus, S. (2012b). TLR signaling in mast cells. Frontiers in Immunology, 3, 185. https://doi.org/10.3389/fimmu.2012.00185

Sansbury, B. E., \& Spite, M. (2016). Resolution of acute inflammation and the role of resolvins in immunity, thrombosis, and vascular biology. In Circulation Research. 
https://doi.org/10.1161/CIRCRESAHA.116.307308

Sato, M., Suemori, H., Hata, N., Asagiri, M., Ogasawara, K., Nakao, K., Nakaya, T., Katsuki, M., Noguchi, S., Tanaka, N., \& Taniguchi, T. (2000). Distinct and essential roles of transcription factors IRF-3 and IRF-7 in response to viruses for IFN- $\alpha / \beta$ gene induction. Immunity. https://doi.org/10.1016/S1074-7613(00)00053-4

Savill, J., Dransfield, I., Gregory, C., \& Haslett, C. (2002). A blast from the past: Clearance of apoptotic cells regulates immune responses. In Nature Reviews Immunology. https://doi.org/10.1038/nri957

Schapiro, D., Jackson, H. W., Raghuraman, S., Fischer, J. R., Zanotelli, V. R. T., Schulz, D., Giesen, C., Catena, R., Varga, Z., \& Bodenmiller, B. (2017). histoCAT: analysis of cell phenotypes and interactions in multiplex image cytometry data. Nature Methods, 14(9), 873-876. https://doi.org/10.1038/nmeth.4391

Schenk, M., Fabri, M., Krutzik, S. R., Lee, D. J., Vu, D. M., Sieling, P. A., Montoya, D., Liu, P. T., \& Modlin, R. L. (2014). Interleukin-1 $\beta$ triggers the differentiation of macrophages with enhanced capacity to present mycobacterial antigen to T cells. Immunology. https://doi.org/10.1111/imm.12167

Schett, G. (2019). Resolution of inflammation in arthritis. In Seminars in Immunopathology. https://doi.org/10.1007/s00281-019-00768-x

Schmitt, H., Neufert, C., Neurath, M. F., \& Atreya, R. (2019). Resolution of Crohn's disease. In Seminars in Immunopathology. https://doi.org/10.1007/s00281-019-00756-1

Schneider, W. M., Chevillotte, M. D., \& Rice, C. M. (2014). Interferon-stimulated genes: A complex web of host defenses. Annual Review of Immunology, 32(1), 513-545. https://doi.org/10.1146/annurev-immunol-032713-120231

Scholten, J., Hartmann, K., Gerbaulet, A., Krieg, T., Müller, W., Testa, G., \& Roers, A. (2008). Mast cell-specific Cre/loxP-mediated recombination in vivo. Transgenic Research, 17(2), 307-315. https://doi.org/10.1007/s11248-007-9153-4

Schroder, K., Hertzog, P. J., Ravasi, T., \& Hume, D. A. (2004). Interferon-Y: an overview of signals, mechanisms and functions. Journal of Leukocyte Biology, 75(2), 163-189. https://doi.org/10.1189/jlb.0603252

Schüffler, P. J., Schapiro, D., Giesen, C., Wang, H. A. O., Bodenmiller, B., \& Buhmann, J. M. (2015). Automatic single cell segmentation on highly multiplexed tissue images. Cytometry Part A, 87(10), 936-942. https://doi.org/10.1002/cyto.a.22702

Seo, S. U., Kwon, H. J., Ko, H. J., Byun, Y. H., Seong, B. L., Uematsu, S., Akira, S., \& Kweon, M. N. (2011). Type I interferon signaling regulates Ly6Chi monocytes and neutrophils during acute viral pneumonia in mice. PLoS Pathogens. https://doi.org/10.1371/journal.ppat.1001304

Serhan, C. N., Brain, S. D., Buckley, C. D., Gilroy, D. W., Haslett, C., O’Neill, L. A. J., Perretti, M., Rossi, A. G., \& Wallace, J. L. (2007). Resolution of in flammation: state of the art, definitions and terms. The FASEB Journal, 21(2), 325-332. https://doi.org/10.1096/fj.06-7227rev

Serhan, C. N., Hamberg, M., \& Samuelsson, B. (1984). Lipoxins: Novel series of biologically active compounds formed from arachidonic acid in human leukocytes. Proceedings of the National Academy of Sciences of the United States of America. https://doi.org/10.1073/pnas.81.17.5335

Serhan, C. N., Yacoubian, S., \& Yang, R. (2008). Anti-Inflammatory and Proresolving Lipid Mediators. Annual Review of Pathology: Mechanisms of Disease, 3(1), 279-312. https://doi.org/10.1146/annurev.pathmechdis.3.121806.151409

Sharma, S., TenOever, B. R., Grandvaux, N., Zhou, G. P., Lin, R., \& Hiscott, J. (2003). Triggering the interferon antiviral response through an IKK-related pathway. Science. https://doi.org/10.1126/science.1081315

Sheppard, P., Kindsvogel, W., Xu, W., Henderson, K., Schlutsmeyer, S., Whitmore, T. E., Kuestner, R., Garrigues, U., Birks, C., Roraback, J., Ostrander, C., Dong, D., Shin, J., Presnell, S., Fox, B., Haldeman, B., Cooper, E., Taft, D., Gilbert, T., ... Klucher, K. M. (2003). IL-28, IL-29 and their class II cytokine receptor IL-28R. Nature Immunology, 4(1), 63-68. https://doi.org/10.1038/ni873

Shibata, T., Motoi, Y., Tanimura, N., Yamakawa, N., Akashi-takamura, S., \& Miyake, K. 
(2011). Intracellular TLR4/MD-2 in macrophages senses Gram-negative bacteria and induces a unique set of LPS-dependent genes. International Immunology.

https://doi.org/10.1093/intimm/dxr044

Silva, M. T. (2010). When two is better than one: macrophages and neutrophils work in concert in innate immunity as complementary and cooperative partners of a myeloid phagocyte system. Journal of Leukocyte Biology. https://doi.org/10.1189/jlb.0809549

Silva, M. T., \& Correia-Neves, M. (2012). Neutrophils and macrophages: The main partners of phagocyte cell systems. Frontiers in Immunology. https://doi.org/10.3389/fimmu.2012.00174

Skaff, O., Pattison, D. I., \& Davies, M. J. (2008). The vinyl ether linkages of plasmalogens are favored targets for myeloperoxidase-derived oxidants: A kinetic study. Biochemistry. https://doi.org/10.1021/bi800786q

Sommereyns, C., Paul, S., Staeheli, P., \& Michiels, T. (2008). IFN-lambda (IFN- $\lambda$ ) is expressed in a tissue-dependent fashion and primarily acts on epithelial cells in vivo. PLoS Pathogens. https://doi.org/10.1371/journal.ppat.1000017

Sonoda, S., Sonoda, T., Nakano, T., Kanayama, Y., Kanakura, Y., Asai, H., Yonezawa, T., \& Kitamura, Y. (1986). Development of mucosal mast cells after injection of a single connective tissue-type mast cell in the stomach mucosa of genetically mast celldeficient W/Wv mice. Journal of Immunology (Baltimore, Md. : 1950).

Sonoda, T., Kanayama, Y., Hara, H., Hayashi, C., Tadokoro, M., Yonezawa, T., \& Kitamura, Y. (1984). Proliferation of peritoneal mast cells in the skin of W/Wv mice that genetically lack mast cells. Journal of Experimental Medicine. https://doi.org/10.1084/jem.160.1.138

Stack, J., Doyle, S. L., Connolly, D. J., Reinert, L. S., O'Keeffe, K. M., McLoughlin, R. M., Paludan, S. R., \& Bowie, A. G. (2014). TRAM Is Required for TLR2 Endosomal Signaling to Type I IFN Induction. The Journal of Immunology. https://doi.org/10.4049/jimmunol.1401605

Stanifer, M. L., Pervolaraki, K., \& Boulant, S. (2019). Differential regulation of type I and type III interferon signaling. In International Journal of Molecular Sciences. https://doi.org/10.3390/ijms20061445

Stock, A. T., Smith, J. M., \& Carbone, F. R. (2014). Type I IFN suppresses Cxcr2 driven neutrophil recruitment into the sensory ganglia during viral infection. Journal of Experimental Medicine. https://doi.org/10.1084/jem.20132183

Stone, K. D., Prussin, C., \& Metcalfe, D. D. (2010). IgE, mast cells, basophils, and eosinophils. Journal of Allergy and Clinical Immunology, 125(2), S73-S80. https://doi.org/10.1016/j.jaci.2009.11.017

Sueltenfuss, E. A., \& Pollard, M. (1963). Cytochemical assay of interferon produced by duck hepatitis virus. Science. https://doi.org/10.1126/science.139.3555.595

Szanto, A., Balint, B. L., Nagy, Z. S., Barta, E., Dezso, B., Pap, A., Szeles, L., Poliska, S., Oros, M., Evans, R. M., Barak, Y., Schwabe, J., \& Nagy, L. (2010). STAT6 transcription factor is a facilitator of the nuclear receptor PPARy-regulated gene expression in macrophages and dendritic cells. Immunity. https://doi.org/10.1016/j.immuni.2010.11.009

Takano, T., Azuma, N., Satoh, M., Toda, A., Hashida, Y., Satoh, R., \& Hohdatsu, T. (2009). Neutrophil survival factors (TNF-alpha, GM-CSF, and G-CSF) produced by macrophages in cats infected with feline infectious peritonitis virus contribute to the pathogenesis of granulomatous lesions. Archives of Virology. https://doi.org/10.1007/s00705-009-0371-3

Takeda, K., \& Akira, S. (2005). Toll-like receptors in innate immunity. In International Immunology. https://doi.org/10.1093/intimm/dxh186

Takeda, K., Kaisho, T., \& Akira, S. (2003). T<scp >OLL $</$ scp $>-L<$ scp $>$ IKE $</$ scp $>R$ $<$ scp $>$ ECEPTORS</scp $>$. Annual Review of Immunology, 21(1), 335-376. https://doi.org/10.1146/annurev.immunol.21.120601.141126

Takeda, K., \& Yamamoto, M. (2010). Current views of toll-like receptor signaling pathways. In Gastroenterology Research and Practice. https://doi.org/10.1155/2010/240365

Takeuchi, O., \& Akira, S. (2010). Pattern Recognition Receptors and Inflammation. In Cell (Vol. 140, Issue 6, pp. 805-820). Elsevier. https://doi.org/10.1016/j.cell.2010.01.022 
Tall, A. R., \& Yvan-Charvet, L. (2015). Cholesterol, inflammation and innate immunity. In Nature Reviews Immunology. https://doi.org/10.1038/nri3793

Tanimura, N., Saitoh, S., Matsumoto, F., Akashi-Takamura, S., \& Miyake, K. (2008). Roles for LPS-dependent interaction and relocation of TLR4 and TRAM in TRIF-signaling. Biochemical and Biophysical Research Communications, 368(1), 94-99. https://doi.org/10.1016/j.bbrc.2008.01.061

Tarighi, N., Menger, D., Pierre, S., Kornstädt, L., Thomas, D., Ferreirós, N., Nüsing, R. M., Geisslinger, G., \& Scholich, K. (2019). Thromboxane-Induced a-CGRP Release from Peripheral Neurons Is an Essential Positive Feedback Loop in Capsaicin-Induced Neurogenic Inflammation. The Journal of Investigative Dermatology, 139(3), 656-664. https://doi.org/10.1016/j.jid.2018.10.011

Taylor, P. R., Brown, G. D., Reid, D. M., Willment, J. A., Martinez-Pomares, L., Gordon, S., \& Wong, S. Y. C. (2002). The $\beta$-Glucan Receptor, Dectin-1, Is Predominantly Expressed on the Surface of Cells of the Monocyte/Macrophage and Neutrophil Lineages. The Journal of Immunology. https://doi.org/10.4049/jimmunol.169.7.3876

Terzić, J., Grivennikov, S., Karin, E., \& Karin, M. (2010). Inflammation and Colon Cancer. Gastroenterology. https://doi.org/10.1053/j.gastro.2010.01.058

Theoharides, T. C., Alysandratos, K. D., Angelidou, A., Delivanis, D. A., Sismanopoulos, N., Zhang, B., Asadi, S., Vasiadi, M., Weng, Z., Miniati, A., \& Kalogeromitros, D. (2012). Mast cells and inflammation. In Biochimica et Biophysica Acta - Molecular Basis of Disease. https://doi.org/10.1016/j.bbadis.2010.12.014

Treutlein, E.-M., Kern, K., Weigert, A., Tarighi, N., Schuh, C.-D., Nüsing, R. M., Schreiber, Y., Ferreirós, N., Brüne, B., Geisslinger, G., Pierre, S., \& Scholich, K. (2018). The prostaglandin E2 receptor EP3 controls CC-chemokine ligand 2-mediated neuropathic pain induced by mechanical nerve damage. Journal of Biological Chemistry, 293(25), 9685-9695. https://doi.org/10.1074/jbc.RA118.002492

Tsai, M., Grimbaldeston, M., \& Galli, S. J. (2011). Mast Cells and Immunoregulation/Immunomodulation. In Advances in experimental medicine and biology (Vol. 716, pp. 186-211). https://doi.org/10.1007/978-1-4419-9533-9_11

Tsalamandris, S., Antonopoulos, A. S., Oikonomou, E., Papamikroulis, G. A., Vogiatzi, G., Papaioannou, S., Deftereos, S., \& Tousoulis, D. (2019). The role of inflammation in diabetes: Current concepts and future perspectives. In European Cardiology Review. https://doi.org/10.15420/ecr.2018.33.1

Uyangaa, E., Kim, J. H., Patil, A. M., Choi, J. Y., Kim, S. B., \& Eo, S. K. (2015). Distinct Upstream Role of Type I IFN Signaling in Hematopoietic Stem Cell-Derived and Epithelial Resident Cells for Concerted Recruitment of Ly-6Chi Monocytes and NK Cells via CCL2-CCL3 Cascade. PLoS Pathogens. https://doi.org/10.1371/journal.ppat.1005256

v. Recklinghausen, F. (1863). Ueber Eiter- und Bindegewebskörperchen. Archiv Für Pathologische Anatomie Und Physiologie Und Für Klinische Medicin. https://doi.org/10.1007/BF01930779

Viola, A., Munari, F., Sánchez-Rodríguez, R., Scolaro, T., \& Castegna, A. (2019). The metabolic signature of macrophage responses. In Frontiers in Immunology. https://doi.org/10.3389/fimmu.2019.01462

Virchow, R. (1989). As Based upon Physiological and Pathological Histology: CELLULAR PATHOLOGY. Nutrition Reviews. https://doi.org/10.1111/j.1753-4887.1989.tb02747.x

Voehringer, D., Liang, H.-E., \& Locksley, R. M. (2008). Homeostasis and Effector Function of Lymphopenia-Induced "Memory-Like" T Cells in Constitutively T Cell-Depleted Mice. The Journal of Immunology. https://doi.org/10.4049/jimmunol.180.7.4742

Vogel, S. N., \& Fertsch, D. (1984). Endogenous interferon production by endotoxinresponsive macrophages provides an autostimulatory differentiation signal. Infection and Immunity. https://doi.org/10.1128/iai.45.2.417-423.1984

Wainszelbaum, M. J., Proctor, B. M., Pontow, S. E., Stahl, P. D., \& Barbieri, M. A. (2006). IL4/PGE2 induction of an enlarged early endosomal compartment in mouse macrophages is Rab5-dependent. Experimental Cell Research, 312(12), 2238-2251. https://doi.org/10.1016/j.yexcr.2006.03.025 
Walker, B. E. (1961). Mast cell turn-over in adult mice. Nature. https://doi.org/10.1038/192980a0

Wang, W., Yin, Y., Xu, L., Su, J., Huang, F., Wang, Y., Boor, P. P. C., Chen, K., Wang, W., Cao, W., Zhou, X., Liu, P., Van Der Laan, L. J. W., Kwekkeboom, J., Peppelenbosch, M. P., \& Pan, Q. (2017). Unphosphorylated ISGF3 drives constitutive expression of interferon-stimulated genes to protect against viral infections. Science Signaling. https://doi.org/10.1126/scisignal.aah4248

Welch, J. S., Ricote, M., Akiyama, T. E., Gonzalez, F. J., \& Glass, C. K. (2003). PPARy and PPARס negatively regulate specific subsets of lipopolysaccharide and IFN-y target genes in macrophages. Proceedings of the National Academy of Sciences of the United States of America. https://doi.org/10.1073/pnas.1031789100

Welle, M. (1997). Development, significance, and heterogeneity of mast cells with particular regard to the mast cell-specific proteases chymase and tryptase. In Journal of Leukocyte Biology. https://doi.org/10.1002/jlb.61.3.233

Wernersson, S., \& Pejler, G. (2014). Mast cell secretory granules: armed for battle. Nature Reviews Immunology, 14(7), 478-494. https://doi.org/10.1038/nri3690

Wu, J., Zhang, Y., Xin, Z., \& Wu, X. (2015). The crosstalk between TLR2 and NOD2 in Aspergillus fumigatus keratitis. Molecular Immunology. https://doi.org/10.1016/j.molimm.2014.11.021

Wu, R., Chen, F., Wang, N., Tang, D., \& Kang, R. (2020). ACOD1 in immunometabolism and disease. In Cellular and Molecular Immunology. https://doi.org/10.1038/s41423-0200489-5

Yanai, H., Chiba, S., Hangai, S., Kometani, K., Inoue, A., Kimura, Y., Abe, T., Kiyonari, H., Nishio, J., Taguchi-Atarashi, N., Mizushima, Y., Negishi, H., Grosschedl, R., \& Taniguchi, T. (2018). Revisiting the role of IRF3 in inflammation and immunity by conditional and specifically targeted gene ablation in mice. Proceedings of the National Academy of Sciences of the United States of America. https://doi.org/10.1073/pnas.1803936115

Yang, D., Liang, Y., Zhao, S., Ding, Y., Zhuang, Q., Shi, Q., Ai, T., Wu, S. Q., \& Han, J. (2020). ZBP1 mediates interferon-induced necroptosis. Cellular and Molecular Immunology. https://doi.org/10.1038/s41423-019-0237-x

Yang, Z., \& Marshall, J. S. (2009). Zymosan treatment of mouse mast cells enhances dectin1 expression and induces dectin-1-dependent reactive oxygen species (ROS) generation. Immunobiology. https://doi.org/10.1016/j.imbio.2008.09.002

Yoshimura, A., \& Muto, G. (2010). TGF- $\beta$ Function in Immune Suppression. https://doi.org/10.1007/82_2010_87

Zhang, Q., Bastard, P., Liu, Z., Le Pen, J., Moncada-Velez, M., Chen, J., Ogishi, M., Sabli, I. K. D., Hodeib, S., Korol, C., Rosain, J., Bilguvar, K., Ye, J., Bolze, A., Bigio, B., Yang, R., Arias, A. A., Zhou, Q., Zhang, Y., ... Casanova, J. L. (2020). Inborn errors of type I IFN immunity in patients with life-threatening COVID-19. Science. https://doi.org/10.1126/SCIENCE.ABD4570 


\section{List of own publications}

Kornstädt, L., Pierre, S., Weigert, A., Ebersberger, S., Schäufele, T. J., Kolbinger, A., Schmid, T., Cohnen, J., Brüne, B., Ebersberger, I., Thomas, D., Ferreiro, N., \& Scholich, K. (2021). Bacterial and Fungal Toll-Like Receptor Activation Elicits Type I IFN Responses in Mast Cells. Frontiers in Immunology, 11, 3872. https://doi.org/10.3389/fimmu.2020.607048

Hahnefeld, L. ${ }^{*}$ Kornstädt, L. ${ }^{*}$ Kratz, D., Ferreirós, N., Geisslinger, G., Pierre, S., \& Scholich, K. (2021). Lipidomic analysis of local inflammation models shows a specific systemic acute phase response to lipopolysaccharides. Biochimica et Biophysica Acta (BBA) - Molecular and Cell Biology of Lipids, 1866(1), 158822. https://doi.org/10.1016/j.bbalip.2020.158822 * equally contributed.

Cohnen, J., Kornstädt, L., Hahnefeld, L., Ferreiros, N., Pierre, S., Koehl, U., Deller, T., Geisslinger, G., \& Scholich, K. (2020). Tumors Provoke Inflammation and Perineural Microlesions at Adjacent Peripheral Nerves. Cells. https://doi.org/10.3390/cells9020320

Tarighi, N., Menger, D., Pierre, S., Kornstädt, L., Thomas, D., Ferreirós, N., Nüsing, R. M., Geisslinger, G., \& Scholich, K. (2019). Thromboxane-Induced a-CGRP Release from Peripheral Neurons Is an Essential Positive Feedback Loop in Capsaicin-Induced Neurogenic Inflammation. The Journal of Investigative Dermatology, 139(3), 656-664. https://doi.org/10.1016/j.jid.2018.10.011 